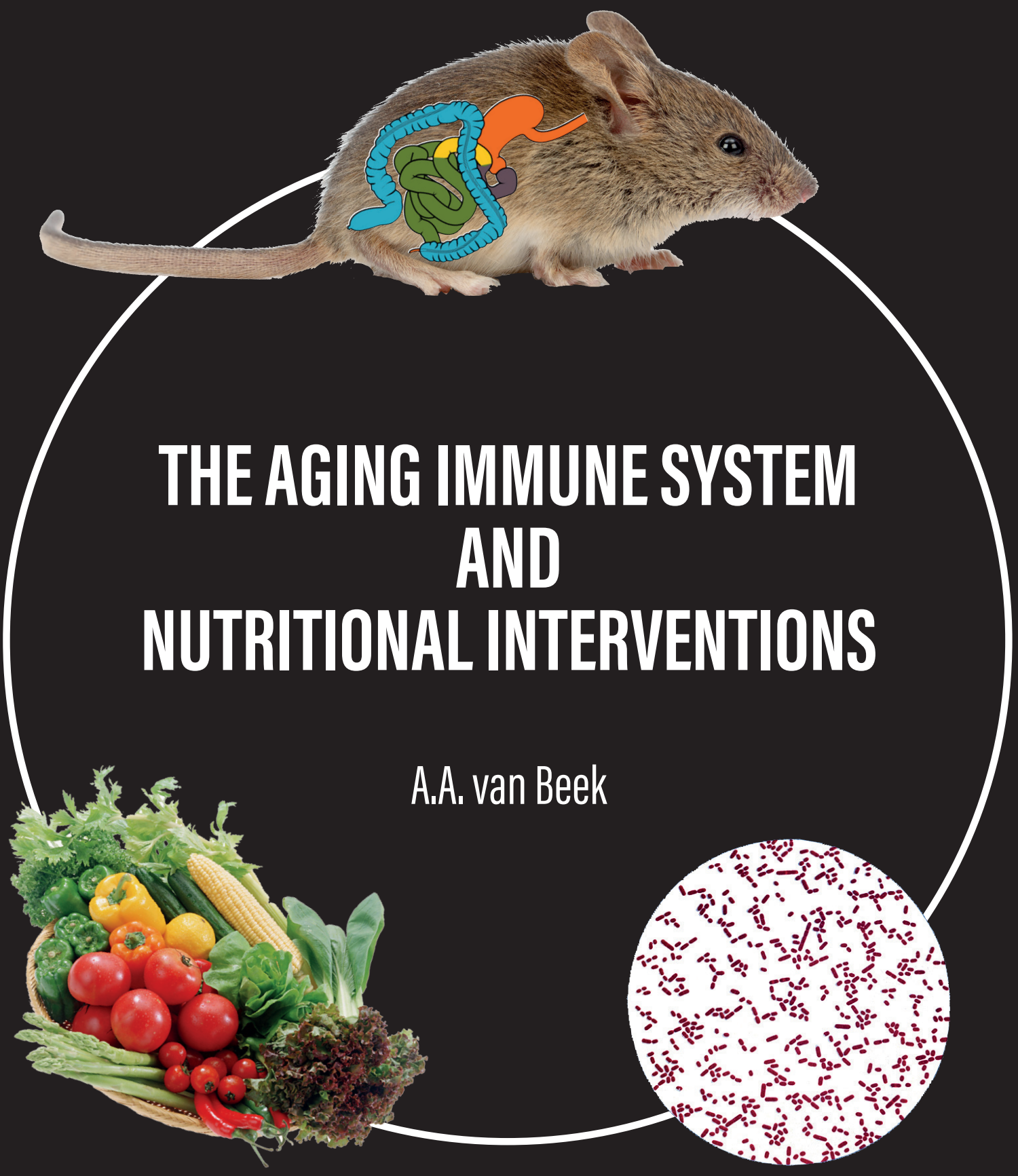




\section{Propositions}

1. Dietary tryptophan restriction arrests B cell development, potentially mediated by gut microbial changes.

(this thesis)

2. The definition of probiotics by the World Health Organization/Food and Agriculture Organization is misleading in that it omits the health condition and age of the host.

(this thesis)

3. Scientists that have overlapping interests and are based at the same academic institute should collaborate rather than compete to optimize scientific yield.

4. Generating a PhD thesis induces stress and hence accelerates the aging process of the PhD student.

5. The successful scientist seems to be the one that is involved more in political issues rather than in scientific issues.

6. The ultimate consequence of reasoning is the realization that there are many things beyond it, including supernatural things. (based on Blaise Pascal, 'Pensées')

Propositions belonging to the thesis, entitled:

'The aging immune system and nutritional interventions'

Adriaan van Beek

Wageningen, 18 January 2017 


\section{The aging immune system and nutritional interventions}




\section{Thesis committee}

\section{Promotors}

Prof. Dr H.F.J. Savelkoul

Professor of Cell Biology and Immunology

Wageningen University

Prof. Dr R.W. Hendriks

Professor of Pulmonary Medicine

Erasmus University Medical Center, Rotterdam

\section{Co-promotors}

Dr P.J.M. Leenen

Associate professor, Department of Immunology

Erasmus University Medical Center, Rotterdam

Prof. Dr H.J. Wichers

Professor of Immune Modulation by Food

Wageningen University

\section{Other members}

Prof. Dr S. Kersten, Wageningen University

Dr A. Mercenier, Nestlé Research Center, Lausanne, Switzerland

Prof. Dr J. Garssen, Utrecht University/Danone Research Center, Utrecht

Prof. Dr C. Nicoletti, Institute of Food Research, Norwich, United Kingdom

This research was conducted under the auspices of the Graduate School Wageningen Institute of Animal Sciences 


\title{
The aging immune system and nutritional interventions
}

\author{
Adriaan A. van Beek
}

Thesis

submitted in fulfillment of the requirements for the degree of doctor at Wageningen University by the authority of the Rector Magnificus Prof. Dr A.P.J. Mol, in the presence of the

Thesis Committee appointed by the Academic Board to be defended in public on Wednesday 18 January 2017 at 4 p.m. in the Aula 
Adriaan van Beek

The aging immune system and nutritional interventions 250 pages.

PhD thesis, Wageningen University, Wageningen, NL (2017) With references, with summary in English

ISBN: 978-94-6257-955-2

DOI: http://dx.doi.org/10.18174/392235 


\section{Table of contents}

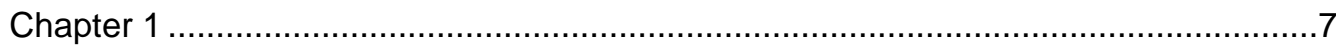

General introduction

Chapter 2 .

The interaction between basophils, T cells and dendritic cells

Chapter 3

Basophil differentiation changes during aging in mice under the influence of senescing microbiota

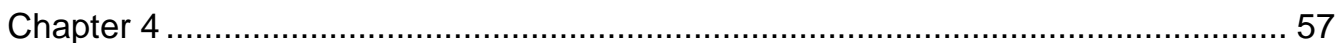

Metabolic and epigenetic alterations in aging macrophages: a recipe for inflammaging?

Chapter 5 73

Interaction of mouse splenocytes and macrophages with bacterial strains in vitro: the effect of age in the immune response

Chapter 6 97

Supplementation with Lactobacillus plantarum WCFS1 prevents age-related decline of mucus barrier in colon of accelerated aging $\operatorname{Ercc}^{-/ \Delta 7}$ mice

Chapter 7

Tryptophan restriction arrests B cell development and enhances microbial diversity in WT and prematurely aging $E r C C 1^{-1 \Delta 7}$ mice

Chapter 8 151

Aged gut microbiota contribute to systemical inflammaging

Chapter 9

General discussion

References

Summary

Acknowledgements (dankwoord)

Curriculum vitae

List of publications 



\section{Chapter 1}

\section{General introduction}

A.A. van Beek 
Chapter 1 


\section{Extended lifespan: towards healthy aging}

The average lifetime increased during the last century, with an average global increase in life expectancy of more than 10 years between 1970 and 2010 [4]. The proportion of individuals aged 60 years and older is estimated to increase from $11 \%$ in 2008 to more than $20 \%$ of the world population by 2050 [5]. About half of the total global deaths occurred among elderly people ( $\geq 65$-year-old) in 2010 [4]. Aging is considered to be part of the normal biological cycle, and possibilities to extend the lifespan by preventing or reverting age-related changes have been studied since decades [11]. This 'luxury' of life extension, however, presents major challenges to society and public health care [12-14], because life extension is accompanied by an increase in cancer and chronic (inflammatory) diseases. Two out of three elderly people in the United States have multiple chronic diseases [17]. Common age-related diseases and their economic impact are listed in Table 1.

Table 1. Common age-related diseases or conditions, their cause and/or risk factors, the role of the immune system in each of these diseases, and estimated economic impact.

\begin{tabular}{|c|c|c|c|c|c|}
\hline Disease & $\begin{array}{l}\text { Cause of } \\
\text { death rank }\end{array}$ & Costs $^{1}$ & Cause/risk factors ${ }^{2}$ & $\begin{array}{l}\text { Immune system } \\
\text { involvement }\end{array}$ & Ref. \\
\hline CVD & 1 & 445 & Diet and lifestyle & $\begin{array}{l}\text { Endothelial dysfunction } \\
\text { and inflammation }\end{array}$ & {$[18,19]$} \\
\hline Cancer & 2 & 125 & $\begin{array}{l}\text { Chemicals, diet and } \\
\text { lifestyle, hormones, } \\
\text { infection, physical } \\
\text { agents, pollution, } \\
\text { radiation }\end{array}$ & $\begin{array}{l}\text { Immune escape } \\
\text { Immuno-editing }\end{array}$ & [20-22] \\
\hline COPD & $3^{3}$ & 50 & $\begin{array}{l}\text { Chemicals, diet } \\
\text { and lifestyle, pollution }\end{array}$ & Inflammation & {$[23,24]$} \\
\hline$A D$ & 6 & $226^{4}$ & Amyloid- $\beta$ aggregates & Microglia dysregulation & [25-28] \\
\hline T2D & 7 & $245^{5}$ & Diet and lifestyle & $\begin{array}{l}\text { Inflammation } \\
\text { (auto-inflammatory) }\end{array}$ & [29-31] \\
\hline PD & 14 & 34 & Idiopathic & Inflammation & {$[32,33]$} \\
\hline Arthritis & - & 128 & Diet and lifestyle & $\begin{array}{l}\text { Inflammation } \\
\text { (incl. autoimmune) }\end{array}$ & {$[34,35]$} \\
\hline Osteoporosis & - & 22 & $\begin{array}{l}\text { Diet and lifestyle, } \\
\text { hormones }\end{array}$ & Osteoclast activation & {$[36,37]$} \\
\hline Sarcopenia & - & 18.5 & $\begin{array}{l}\text { Diet and lifestyle, } \\
\text { hormones, increased } \\
\text { fat mass and } \\
\text { insulin resistance }\end{array}$ & $\begin{array}{l}\text { Elevated circulating } \\
\text { cytokines }\end{array}$ & [38-40] \\
\hline
\end{tabular}

${ }^{1}$ Annual costs in the USA only, and expressed in billion US dollars. All cost estimates are from data between 2000-2015 and include direct and indirect costs; ${ }^{2}$ Genetics and aging were not accounted for, as they are shared risk factors for any of the listed diseases; ${ }^{3}$ Includes other chronic lung diseases; ${ }^{4}$ Includes other dementias; ${ }^{5}$ Includes type 1 diabetes (accounting for $5 \%$ of total diabetes patients). Abbreviations used: AD = Alzheimer's disease; $\mathrm{COPD}=$ chronic obstructive pulmonary disease $; \mathrm{CVD}=$ cardiovascular disease; $\mathrm{PD}$ $=$ Parkinson's disease; $\mathrm{T} 2 \mathrm{D}=$ type 2 diabetes . 
Chapter 1

In total, these chronic diseases pose an economic burden to the United States of more than 1 trillion US dollars per year. In addition to age-related diseases, a reduced efficacy of vaccinations and an increased susceptibility to infections occur, which all together negatively impact quality of life and further increase the economic burden [4143]. Improving the health span (also defined as 'healthy aging') should therefore be pursued rather than or simultaneous with extended lifespan [44].

The immune system is involved in many age-related diseases (Table 1). The immune system becomes deregulated with age, developing a low-grade inflammation ('inflammaging'). Because the immune system protects organisms against all types of pathogens and deteriorates with aging, it is crucial to find applicable interventions to reinforce immunity. Interventions aimed at the immune system will contribute to solve the 'luxury problem' of aging, and support healthy aging.

This thesis will focus on the effects of immune aging ('immunosenescence') and the application of treatments to enhance the function of the aging immune system.

\section{Causes of aging}

In order to apply treatments that enhance the aging immune system, it is important to understand the causes of aging. Lopéz-Otin et al (2013) defined nine hallmarks of aging: genomic instability, telomere attrition, epigenetic alterations, loss of protein homeostasis, deregulated nutrient sensing, mitochondrial dysfunction, cellular senescence, stem cell exhaustion, and altered intercellular communication [11].

DNA becomes increasingly susceptible to damage and degradation due to the shortening of telomeres, resulting in widespread tissue atrophy with severe depletion of tissue stem-cell reserves $[45,46]$. Shortening of telomeres activates cellular growth arrest, senescence, and apoptosis, and induces metabolic and mitochondrial compromise [47]. An increased frequency of chromosome translocations causes a higher risk of transformation. Via tumor suppressor genes, cells can prevent transformation by undergoing senescence. In the senescent state, cells do not divide anymore but remain metabolically active $[45,48,49]$.

Mitochondrial DNA mutations due to high oxidative stress lead to damage and death in various types of cells [50], including hematopoietic stem cells (HSC) [45]. Oxidative stress causes DNA damage, but is also induced by DNA damage [51].

DNA repair mechanisms detect DNA damage and execute DNA repair. Insufficient DNA repair capacity (and hence accumulation of DNA damage) results in genomic instability, cellular senescence, and loss of various stem-cell compartments [52].

In many cell types, epigenetic regulation has been found to play a pivotal role in aging [53]. Epigenetic modifications include DNA methylation and histone modifications [54]. Significant variation was found in the extent of aging between co-housed animals [55], 
implying epigenetic differences. The epigenetic profiles of monozygotic twins were the same as infants but differed in later life [56], indicating that epigenetic alterations are induced by environmental factors. The variation in human immunity is indeed largely driven by non-heritable factors [57].

In summary, nuclear DNA damage is described to be a master regulator of all hallmarks of aging [58].

\section{Accelerated aging mouse model: $\operatorname{Ercc1}^{-/ \Delta 7}$ mice}

DNA repair deficiency results in accelerated aging [59]. Accelerated aging animal models have been developed to mimic human progeroid syndromes like Cockayne syndrome (CS), trichothiodystrophy (TTD), and XpF-Ercc1 (XFE) progeroid syndrome [58]. The $E r c c 1^{-1 \Delta 7}$ mouse model has been described to 'develop the widest spectrum of bona fide aging-associated phenotypes and pathology that is commonly observed in elderly humans' [58]. ERCC1 is a central protein in DNA repair. Significantly hampered DNA repair capacity in Ercc1-deficient mice $\left(\mathrm{ErCC1}^{-1}\right)$, therefore, renders them sensitive to agents inducing DNA damage [60], including dietary components. By introducing the $\Delta 7$ allele, lacking the code for the final 7 amino acids of the Ercc1 protein, DNA repair capacity is partly retained, resulting in a median lifespan of 20 weeks in $\mathrm{ErCC1}^{-/ \Delta 7}$ mice compared with 3-4 weeks in $\operatorname{Ercc1}^{-/}$mice $[61,62]$.

\section{The aging immune system}

The immune system is divided into an innate and an adaptive compartment. Cellular components of both compartments are derived from HSC (Figure 1). The innate part of the immune system originates predominantly from the common myeloid progenitors (CMP), which develop into granulocytes, monocytes, and macrophages. The adaptive part originates from the common lymphoid progenitors (CLP), which give rise to $T$ and B cells. CLP, however, also give rise to innate lymphoid cells (ILC), which do no express rearranged antigen receptors like $T$ and $B$ cells do $[45,63]$.

\section{HSC}

HSC numbers in BM increase with age, both in humans and mice [64]. Functional deficits, however, accumulate in aged HSC [65]. HSC from old animals downregulate the expression of genes that control immune cell development, compared with HSC from young animals. This causes an increasing loss of control on the immune cell development in aging [65-67]. The function of aged HSC is further in demise by p53mediated proliferative arrest [45]. Aged HSC do not efficiently generate lymphoid cells, 
whereas the generation of myeloid cells is increased ('myeloid bias') [55, 66, 68]. Thus, HSC acquire intrinsic defects and changes with aging [64].

\section{Humoral factors}

With aging, serum levels of both pro-inflammatory mediators (IL-1 $\beta$, IL-6, TNF, prostaglandin E2) and anti-inflammatory mediators (CRP, IL-1RA, sTNFR) increase [64, 69, 70]. These factors reflect the low-grade inflammation in aging, also called 'inflammaging' [71], and are strong predictors for mortality risk [72]. The simultaneous increase in pro- and anti-inflammatory mediators reflect the deregulation of the immune system with aging.

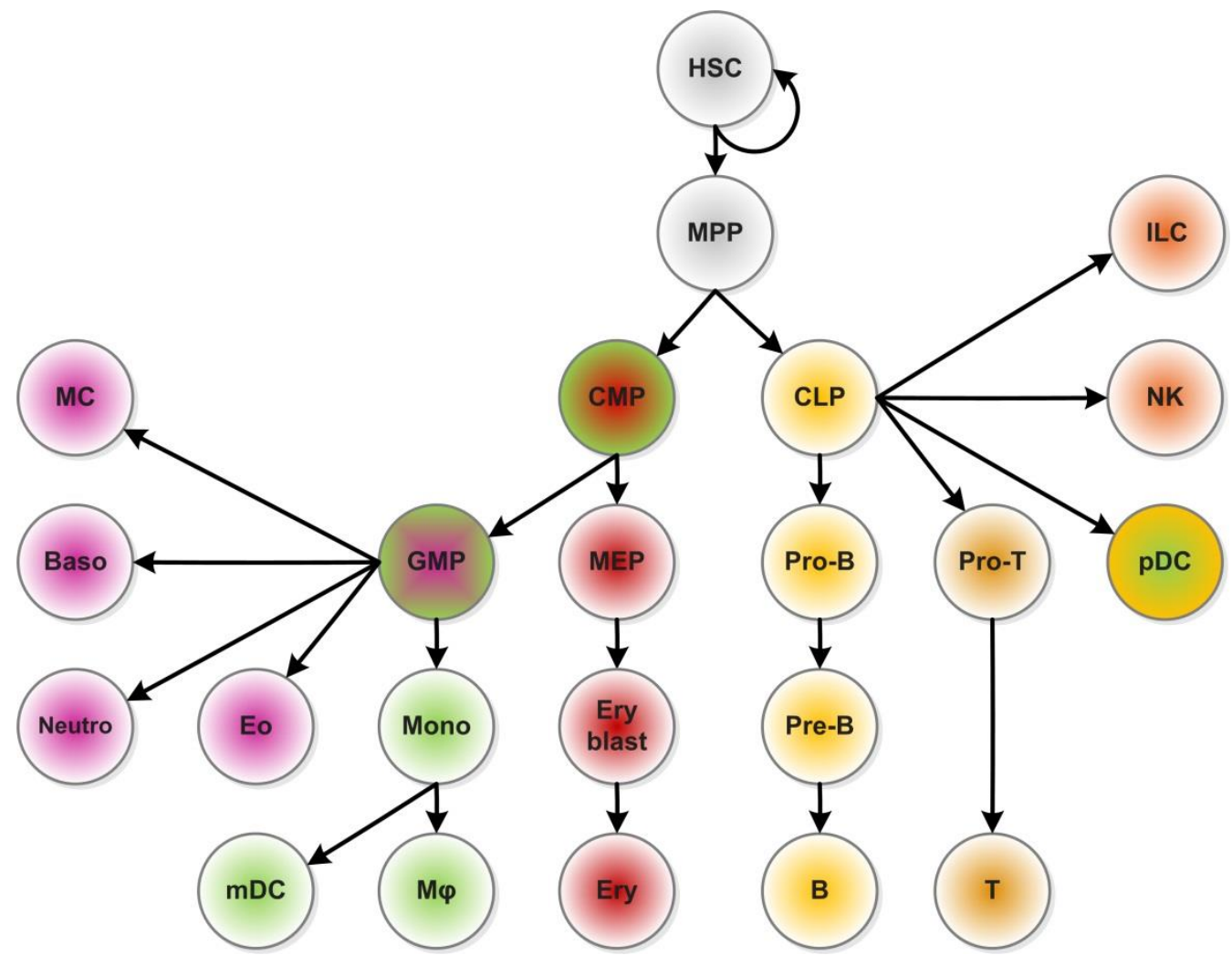

Figure 1. Schematic overview of the hematopoiesis in the bone marrow. In grey non-committed precursor cells. In yellow/orange all lymphoid-committed precursors and differentiated cells. In red the erythroid lineage, in green and purple the myelomonocytic precursors and myeloid cells. B = B cell; Baso = basophil precursor; CLP = common lymphoid progenitor; $\mathrm{CMP}=$ common myeloid progenitor; $\mathrm{Eo}=$ eosinophil; Ery = erythrocyte; Ery blast = erythroblast; GMP = granulocyte-macrophage progenitor; $\mathrm{HSC}=$ hematopoietic stem cell; ILC = innate lymphoid cell; MC = mast cell; MEP = megakaryocyte-erythroid progenitor; MPP = multipotent progenitor; Neutro = neutrophil; NK = natural killer cell; $\mathrm{pDC}=$ plasmacytoid dendritic cell; Pre-B $=$ pre $-\mathrm{B}$ cell; Pro- $\mathrm{B}=$ pro-B cell; Pro- $\mathrm{T}=$ pro- $\mathrm{T}$ cell; $\mathrm{T}=\mathrm{T}$ cell .

\section{Granulocytes}

Granulocytes comprise neutrophils, eosinophils and basophils, and are derived from GMP. Neutrophil numbers progressively increase with age [73]. Increased numbers of 
neutrophils are associated with poorer survival in elderly [74]. The age-related impairment of chemotaxis, signaling pathways, phagocytosis, and ROS production by neutrophils causes a reduced ability to eliminate pathogens and inhibits the interaction with the adaptive immune system [63, 75-77]. Also, the function of eosinophils in elderly seems to be reduced, as IL-5 stimulation of eosinophils derived from elderly resulted in a significantly decreased degranulation [78]. Basophils have been implicated to be antigen-presenting cells (APC), and are discussed in more detail in chapter 2 . In response to anti-lgE, histamine release increased, while in response to $f$-met peptide a decline in histamine release was observed [79].

\section{Monocytes and macrophages}

Monocyte subsets change considerably with age. The subpopulation of classical CD14 ${ }^{\text {hi }}$ CD16 monocytes, in young individuals representing $>95 \%$ of total monocytes, decreases to $<80 \%$, while the non-classical CD16 ${ }^{+}$subsets increase [80-82]. This shift reflects the change also observed in inflammatory conditions. During aging, monocytes and macrophages acquire several defects in phagocytosis and cytokine production [81, 83-88]. The influence of aging on macrophage phenotype and function is discussed in detail in chapter 4 .

\section{Lymphocytes}

Lymphocytes belong to both the innate and the adaptive system. Examples of ILC are natural killer (NK) cells, lymphoid tissue inducer (LTi) cells, and ILC1, ILC2, and ILC3 [89]. CD4 ${ }^{+} \mathrm{T}$ helper (Th) cells, CD8 ${ }^{+}$cytotoxic $\mathrm{T}$ cells (CTL), and CD19+ $\mathrm{B}$ cells belong to the adaptive system. $B$ and $T$ cells are capable to develop long-lasting memory.

With aging, the loss of hematopoietic tissue in the BM is associated with a decline in $B$ cell lymphopoiesis $[49,55,90,91]$. Several studies show a significant decrease in percentages and numbers of CD19 ${ }^{+} B$ cells in blood with age [73, 91-93]. In addition, the accumulation of long-lived $B$ cells in aging inhibits the $B$ cell production [90]. As a consequence, the ratio of naĩve/memory $B$ cells is reduced [94].

In addition to the decreased generation of B cells, functional defects are present in these cells. In general, humoral immune responses are impaired in elderly, possibly by a severely reduced $B$ cell receptor repertoire [95-97]. A decrease in total antibody production, but an increase in auto-antibody production is observed in elderly [97, 98]. Many changes in aging $B$ cells might be related to a decline in the function of or cooperation with $\mathrm{CD}^{+} \mathrm{T}$ helper cells $[99,100]$. Other changes, like a decreased 'capping' activity due to an anomalous cholesterol/phospholipid ratio in the cell membrane, seem to be intrinsic to $B$ cells [12].

Recently, a new subset of IL-10-producing $C D 1 d^{\text {hi }} C D 5^{+}$regulatory $B$ cells (Breg) in mice was discovered [101]. Effector $B$ cells activate and maintain effector $C D 4^{+} T$ 
Chapter 1

cells, presenting antigen to and co-stimulating $T$ cells. Breg reciprocally promote development of $\mathrm{FoxP}^{+}$regulatory $\mathrm{T}$ cells (Treg) and exert similar immune-suppressing activities [101, 102]. The first indication of a change in these cell types during aging was shown by Twohig et al (2009). They reported an increased percentage of Breg in mice and suggested that the expansion suppresses the increasing numbers of autoreactive $B$ cells. The increased percentages of Breg, together with the decrease in function of dendritic cells (DC), may cause a decline in effector $C D 4^{+} T$ cell function [103]. An important macroscopic alteration in the immune system is the atrophy of the thymus (thymic involution), which probably already begins in early childhood [104, 105]. Hence, the production of new $T$ cells declines with age [106], resulting in reduction of naïve $T$ cell repertoire and accumulation of memory $T$ cells [107-109]. Young adults that were thymectomized within 2 weeks after birth, showed a premature immune aging of the immune system, marked by the reduced number of T cells, accumulation of oligoclonal memory $T$ cells, and a pro-inflammatory status [106]. Thus, the thymic involution is not only an anatomical-histological alteration, but also has a profound effect on the efficacy and function of peripheral $T$ cells.

T cell responses in elderly are decreased, which may be related to both cell-intrinsic and cell-extrinsic factors, e.g. diminished capacity of DC [110]. Signal transduction in human $T$ cells from elderly was reduced by a defect in tyrosine phosphorylation of CD3 (TCR), CD4, CD8 or IL-2R [111] and by an altered cell membrane fluidity [13].

A significant increase in $\mathrm{CD} 4^{+} \mathrm{T}$ helper $(\mathrm{Th})$ cells and a decrease of $\mathrm{CD} 8^{+}$cytotoxic $\mathrm{T}$ cells $(C T L)$ was found in aged individuals $[108,109]$. Phenotypic changes in $T$ cells occur with age, e.g. downregulation of costimulatory molecules [112], upregulation of co-inhibitory molecules (KLRG1, CD152/CTLA4) [42], and a decreased expression of alpha-4 (a4) integrin (CD49d), which is important in peri- and extra-vascular lymphocyte trafficking [108]. It is postulated that infection with the CMV virus causes accumulation of CMV-specific terminally differentiated $\mathrm{CD}^{+}$and $\mathrm{CD}^{+} \mathrm{T}$-cells due to repeated reactivation and thereby inducing acceleration of immune senescence. The CTL repertoire thus becomes increasingly skewed towards previously encountered antigens such as cytomegalovirus (CMV), limiting the ability to respond to newly encountered viruses [113-115]. Persistent viral infections, e.g. by CMV or HIV, have a profound effect on the distribution of naïve T cells and memory T cells [76, 106, 116-118].

The Th cell population can be divided into several classes: Tbet $^{+}$Th1, GATA3 ${ }^{+}$Th2, RORyt $^{+}$Th17 cells, and FoxP3 ${ }^{+}$Tregs [119]. Treg regulate the immune response by secreting IL-10 and expressing CD152 (CTLA-4) [120]. Elderly individuals (>65 years) have an increased proportion of peripheral Treg, but the lack of CD127 (IL7Ra) expression on Treg results in a loss-of-function [121]. The number of central Treg (generated in the thymus) is decreased, because of thymic involution [122]. 


\section{The aging gut}

The gut is the largest immune organ and contains about $10^{13}-10^{14}$ bacteria $[123,124]$. Commensal gut microbiota improve epithelial barrier function, inhibit pathogenic bacteria and modulate the immune system [125] and thus contribute to immune homeostasis in the gut [126]. Gut-associated lymphoid tissues (GALT) are organized follicles or patches with immune cells, which include isolated lymphoid follicles and Peyer's patches (PP) [127].

With age, the number of PP regresses. Intestinal $B$ and $T$ cells seem to be relatively unaffected with age [128]. Intestinal secretory $\lg A(\operatorname{sg} A)$ levels in mice are not affected during aging, but the basal production of IL-2 and cytokines that are associated with IgA switching (IL-5 and TGF- $\beta$ ) is reduced in the PP. At the same time, IL-4 production is increased in mesenteric lymph nodes (MLN) [129]. The quality of slgA in elderly is probably diminished, as it is observed that species diversity of protective anaerobes in the gut diminishes $[130,131]$. In addition, age-related differences in gut microbiota composition are found [132, 133]. Higher drug intake, including broadspectrum antibiotics by elderly, has a negative effect on the composition of intestinal microbiota [133]. Biagi et al. (2010) reported that gut microbiota composition differs significantly between centenarians and elderly people (60-80 years old). Specific changes in the relative proportion of Firmicutes subgroups were observed. Furthermore, the gut microbiota of centenarians were enriched in Proteobacteria, so-called pathobionts that are considered to be minor and opportunistic components of the gut inducing pathology under certain circumstances. A rearrangement in the composition of butyrate-producing bacteria in centenarians was found [107] and a reduced colonic short chain fatty acid (SCFA) production was associated with a lower fiber intake and antibiotic treatment [133]. Butyrate is an SCFA, which represents a major energy source for enterocytes, is involved epigenetic regulation, and has been implicated in the protection against inflammatory bowel diseases [107, 132, 134].

A crucial component of the intestinal barrier is secreted mucus consisting of heavily glycosylated proteins that form a firmly adherent layer on top of the colonic epithelium [135]. Absence of mucus, such as in Muc2 ${ }^{-/}$mice, leads to spontaneous development of colitis [136]. Mucus serves as a feeding source for certain types of gut microbiota, and thus regulates the gut microbiota composition and immunity $[137,138]$.

\section{Interventions to prevent or revert aging-related effects on immunity}

Pharmacological

Transplantation of aged mouse thymus into young mice rejuvenates the thymus [139]. Factors like thymic stromal lymphopoietin (TSLP), keratinocyte growth factor (KGF), $\mathrm{GH}$, insulin-like growth factor-1 (IGF-1), acylated ghrelin and IL-7 may play a role in 
Chapter 1

the thymic (and BM) rejuvenation [121, 140,141]. Elderly males undergoing sex steroid ablation for prostate tumor treatment showed increased circulating naïve $T$ cell numbers [142], suggesting the involvement of sex hormones in $T$ cell differentiation. Inhibition of age-related decline in T cell development and function has been achieved by manipulating IL-7, KGF, or growth hormone concentrations in preclinical and clinical trials [49]. B-cell depletion in mice reactivated $B$ lymphopoiesis and rejuvenated the $B$ lineage. The reconstituted B-cell compartment in old mice also partly restored the capacity to mount an antibody response to a new antigenic challenge $[90,143]$. Rheumatoid arthritis and lymphoma patients treated with rituximab (anti-CD20 antibody depleting B cells) show similar improvements [143].

\section{Diet and lifestyle}

Supplementation with retinoic acid, the active metabolite of vitamin A, was found to stimulate neutrophil adhesion and migration function in aged individuals [144]. Daily multivitamin intake or higher intakes of vitamin $\mathrm{C}$ and $\mathrm{E}$ decreased telomere shortening [145]. Lack of minerals like selenium and zinc also contribute to the immunodeficiency of elderly. Supplementation of zinc reconstituted the production of IFN- $\gamma$, and stimulates the production and activity of NK cells and neutrophils [146-148].

Consumption of resveratrol (3,5,4'-trihydroxystilbene, a polyphenol from red grapes) is associated with increased lifespan in many species, inducing the activity of silent mating type information regulation 2 homologue (Sirtuin) proteins. Sirtuins are able to deacetylate histones, are involved in epigenetic regulation, and are upregulated after dietary restriction (DR) [149-152]. A mouse study showed that addition of resveratrol to a high-caloric diet improved health and survival, nearly completely reversing the negative effect of the high-caloric diet [151]. Other plant polyphenols stimulate Sirtuins as well, like butein, piceatannol and quercetin [150].

Furthermore, it was found in a mouse model that thymic involution was inhibited by long-term DR [153]. A 20-year longitudinal study on the effect of DR in rhesus macaques showed a general beneficial effect with regard to aging, i.e. improved survival and a reduced risk to die from age-related diseases [154]. T cell distribution and function in aged DR-primates showed more similarity to young animals than aged animals [155]. Other studies, however, highlighted an increased mortality after influenza infection, impaired NK cell function, and decreased IgA in the guts of aged DR-mice [156158]. In addition to $D R$, regular and moderate physical activity may be beneficial to delay age-related effects, as primary antibody responses were increased [76, 159]. 


\section{Probiotics}

Probiotics are defined by the World Health Organization (WHO) and Food and Agricultural Organization (FAO) as "live microorganisms which when administered in adequate amounts confer a health benefit on the host" [160]. Probiotics are used to improve conditions in gastrointestinal inflammatory diseases [161]. Probiotics compete with other bacteria, including pathogenic species, prime the immune system, influence barrier aspects, and produce short-chain fatty acids (SCFA; Figure 2). Microbeassociated molecular patterns (MAMP) that bind to pattern recognition receptors (PRR) on host immune cells lead to cytokine production by immune cells and maturation or suppression of immune cells [162-164]. Because the aging process is also marked by inflammatory conditions, probiotics might be beneficial to restore the immune balance in aged individuals.

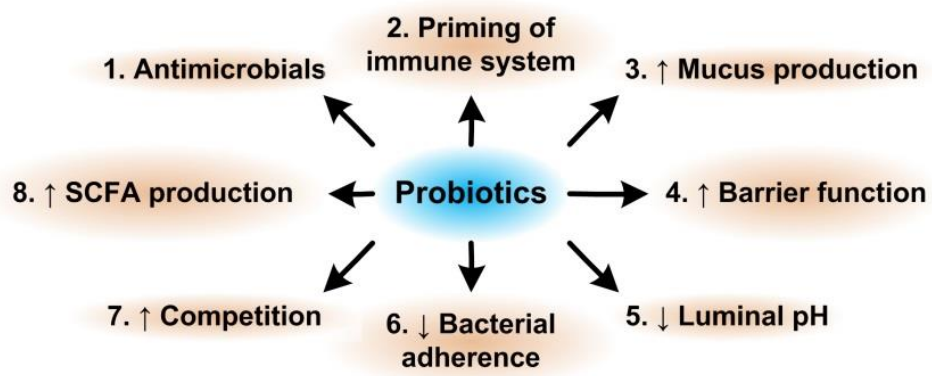

Figure 2. Mechanisms of action by which probiotics modulate microbiota composition and host functions. Probiotic strains can 1) produce antimicrobial compounds; 2) prime the immune system, and induce e.g. regulatory T cells and IgA production; 3) increase mucus production; 4) increase the barrier function by e.g. increasing tight junctions; 5) lower the $\mathrm{pH}$ in the gut lumen; 6) decrease bacterial adherence and translocation; 7) compete with pathogens; and 8) produce short-chain fatty acids (SCFA), which provide energy to epithelial cells.

Long-term supplementation with a Bifidobacterium animalis strain in middle-aged mice extended lifespan, accompanied by reduced tumor and ulcer incidence [165]. Combining this Bifidobacterium strain with arginine supplementation resulted in attenuated TNF and MIP-2 concentrations in the colon [166]. A Lactobacillus fermentum strain reversed the decline in neutrophil phagocytosis and function in aged mice [167]. Administration of Lactobacillus pentosus inhibited NF-KB signaling in aged rats via diminished LPS production by gut microbiota [168].

A probiotic mixture (IRT5) attenuated age-related increase of IL-1 $\beta$ and TNF in the colon of rats [169]. Some probiotic strains showed modulatory effects on the gut microbiota composition of elderly subjects [170, 171], whereas supplementation of Bifidobacterium lactis enhanced phagocytic activity of monocytes and granulocytes in elderly [172]. 
Chapter 1

Thus, probiotic treatment of elderly people may result in the preservation or restoration of a healthy gut microbiota composition and enhancement of immune function.

\section{Dietary tryptophan restriction}

Other selected dietary components that influence health- and lifespan are amino acids, such as methionine [173] and tryptophan [174]. Restriction of tryptophan in the diet resulted in an extension of lifespan and a delay in aging in rodents [174-176]. Tryptophan is an essential amino acid. If the body is in nitrogen balance (i.e. not in growth), most tryptophan is metabolized into ATP, while less than $1 \%$ is used for protein synthesis [177]. A small proportion of dietary tryptophan is metabolized by gut microbiota [177]. In fact, tryptophan is crucial to maintain microbial diversity [178, 179]. DC are able to absorb tryptophan to induce tolerance via inhibition of effector T cells and induction of Treg [180]. Tryptophan breakdown is increased with age [181] and in several autoimmune and neurodegenerative diseases [182, 183]. Decreased serum levels of tryptophan and increased serum levels of kynurenine have been observed in elderly people and were associated with elevated IL-6 levels [184].

\section{Aim of the thesis}

This work was embedded in a larger theme focused on gastrointestinal health, funded by Top Institute of Food and Nutrition (TIFN), and part of project GH002, entitled "Food-induced modulation of the intestinal immune barrier". This theme aimed on developing methods for assessing and understanding the interactions between diets, microbiota, and maintaining homeostasis in the intestinal immune barrier. The project identified the primary sites where the immune system samples immune-active components from the lumen of the gut [185]. In addition, the mechanism by which probiotics modulate the intestinal barrier and immunity was studied [186].

The work presented in this thesis aims to identify major age-related changes in myeloid immune cells (basophils and macrophages) and lymphoid immune cells ( $B$ and $T$ cells) and to find dietary interventions to revert or prevent age-related effects. Chapter 1 introduces the societal relevance of aging research, and summarizes current knowledge about the aging immune system and potential interventions to delay the aging process. In chapter 2, we review current knowledge on basophils as antigen presenting cells. We propose that basophils may act as accessory cells, and hypothesize that basophils can instruct DC. We present evidence for interaction between DC and basophils in vitro. Finally, we highlight as an outstanding question: what is the effect of age on basophils? In chapter $\mathbf{3}$, we therefore set out to study the effect of age on basophils. In addition, we transferred microbiota derived from mice with different ages to germfree mice to assess its effects on basophil phenotype and function. 
Moreover, basophil maturation cultures, as a proxy for basophil precursors, were optimized permitting the assessment of production of relevant cytokines like IL-4. A detailed overview of the available knowledge on aging macrophages, and their putative contribution to inflammaging, is discussed in relation to autophagy, metabolism, epigenetics, and potential (dietary) interventions in chapter 4. Because many potential probiotic strains are available, we set out to investigate the interaction between a number of bacterial strains and immune cells in vitro in chapter 5 . By measuring the cytokine production and surface markers, we assessed how bacterial strains interact with young and aged splenocytes and bone marrow-derived macrophages. Based on IL-10/TNF ratios, we selected three putative probiotic strains to be tested in vivo for delaying age-related effects. Chapter 6 describes the effects of the application of the three selected bacterial strains on the immune system and the gut of accelerated aging $E r c c 1^{-/ \Delta 7}$ mice. We found marked differences between the effects that the three selected bacterial strains had in the aged immune system and gut.

Dietary tryptophan restriction is known to extend lifespan in rodents, but it is unknown what mechanisms underlie life extension. We therefore investigated the effect of dietary tryptophan restriction on immunity and microbiota of wild-type and $E r c c 1^{-/ \Delta 7}$ mice in chapter 7 . We report that dietary tryptophan restriction mostly affected B cells, in comparison to $\mathrm{T}$ cells and myeloid cells. Dietary tryptophan restriction also affected microbiota composition. Because it is known that the microbiota composition changes with age, we studied the role of microbiota on the aging gut and immune system in chapter 8. Gut microbiota from young or aged mice were transferred to germfree recipient mice. Our gene expression, microbiota composition, and immune cell data confirmed that microbiota drive part of the aging phenotype. In chapter $\mathbf{9}$, we evaluate the outcomes of these studies with respect to recent scientific findings in aging research, and discuss the implications of our research for the rational design of intervention strategies and propose future research opportunities. 


\title{
Chapter 2
}

\section{The interaction between basophils, $T$ cells and dendritic cells}

\begin{abstract}
A.A. van Beek ${ }^{1,2}$, E.F. Knol ${ }^{3}$, J.A. Hoogerland ${ }^{2}$, C. Deen ${ }^{2}$, P. de Vos ${ }^{1,4}$, H.F.J. Savelkoul $^{1,2}$, R.J.J. van Neerven ${ }^{2,5}$

${ }^{1}$ Top Institute Food and Nutrition, Wageningen, the Netherlands; ${ }^{2}$ Cell Biology and Immunology Group, Wageningen University, Wageningen, the Netherlands; ${ }^{3}$ Department of Dermatology/Allergology, University Medical Center, Utrecht, the Netherlands; ${ }^{4}$ Pathology and Medical Biology, University of Groningen, Groningen; ${ }^{5}$ Royal FrieslandCampina, Amersfoort, the Netherlands
\end{abstract}

Parts of this chapter have been published as a review in International Archives of Allergy and Immunology; Recent Developments in Basophil Research: Do Basophils Initiate and Perpetuate Type 2 T-helper Cell Responses?

International Archives of Allergy and Immunology 2013; 160(1): 7-17. 
Chapter 2

\section{ABSTRACT}

Basophils account for only $0.1-1 \%$ of all peripheral blood leukocytes. They were considered to be a redundant cell type for a long time. However, several findings show a non-redundant role for basophils in Th2 immune responses in helminth infections, allergy and autoimmunity. Both IgE-dependent and IgE-independent pathways have been described to contribute to basophil activation. In addition, several recent studies reported that basophils can function as antigen presenting cells and are important in initiation of Th2 immune responses. However, there are also conflicting studies that do not corroborate the importance of basophils in Th2 immune responses. This chapter discusses the role of basophils in Th2 immune responses in view of these recent findings. Furthermore, we present evidence for a role for basophils in dendritic cell maturation and function, by co-culturing purified bone marrow-derived basophils and bone marrow-derived dendritic cells. 


\section{INTRODUCTION}

Basophilic granulocytes have been discovered over a century ago [187], but it took more than 9 decades to demonstrate their direct involvement in allergy [188]. Granulocytes are divided in three subsets: basophilic granulocytes, eosinophilic granulocytes and neutrophilic granulocytes. Basophilic granulocytes circulate in the peripheral blood and account for approximately $0.1-1 \%$ of blood leukocytes. They measure $7-10$ $\mu \mathrm{m}$ in diameter, have a segmented nucleus and contain metachromatic granules. Basophils share some features with mast cells, and have often been considered as minor, and possibly redundant, relatives of mast cells or as blood-circulating precursors of tissue-resident mast cells [189]. Even though basophils differ from mast cells in several aspects (see Table 1), they are more conveniently isolated (from the blood) than mast cells (from the tissues), and are often used as a surrogate for mast cells [190]. An important immunological role of basophils emerged when IgE-dependent interleukin (IL)-4 and IL-13 secretion by these cells was discovered (Figure 1) [191194]. More recently, several studies in mouse models were published that indicate that basophils may act as antigen-presenting cells (APCs). In addition, basophils were shown to be involved in inducing and perpetuating Th2 responses. This chapter discusses these recently discovered functions of basophils and adds data on the interaction between basophils and dendritic cells (DC).

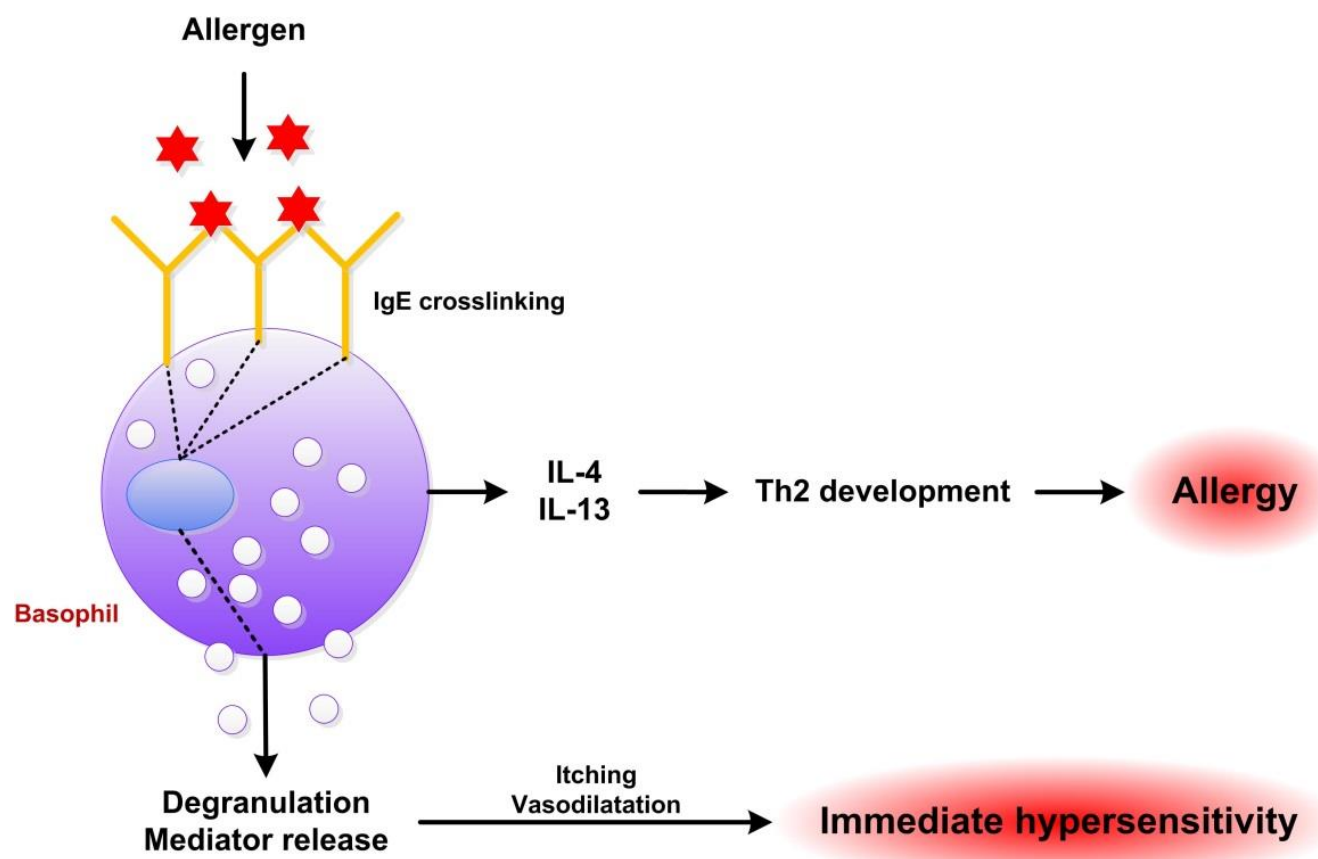

Figure 1. Classical view of basophil in allergy. IgE crosslinking on the basophil by allergens leads to: secretion of IL-4 and IL-13 that enhance the Th2 immune response which is involved in allergy (A); and degranulation and mediator release resulting in immediate hypersensitivity (B). 


\section{Basophil progenitors and differentiation}

Human basophils and mast cells arise from CD34+ granulocyte-monocyte progenitors (GMPs) in the bone marrow (BM). Differentiation and survival of human basophils is mainly dependent on IL-3, IL-5 and granulocyte-macrophage colony stimulating factor (GM-CSF), with IL-3 being 10-50-fold more potent than the other two factors [195, 196]. IL-3 also induces ST2 (IL-33R $\alpha$ ) expression on basophils, leading to enhanced IL-33 responsiveness [197]. The important role of IL-3 is illustrated by the fact that differentiation of human basophils from human cord blood precursors occurs in 3 weeks in the presence of recombinant IL-3 in vitro [198]. Recently, enhanced differentiation, survival and/or activation of basophils has been found under the influence of IL-33 [197] and leptin [199].

Thymic stromal lymphopoietin (TSLP), produced by epithelial cells, stromal cells and mast cells, promotes the expansion of basophils in mice [200-206]. TSLP promotes mouse basophil hematopoiesis and activation independently of IL-3. TSLP-induced basophils are smaller in size than IL-3-stimulated basophils and express higher levels of IL-33R. A role for TSLP in maturation of human basophils has not been shown to date. However, the majority of basophils from healthy human donors express TSLPR. Also, IL-33R levels are significantly higher in human basophils obtained from inflammatory sites, suggesting that TSLP also induces basophil hematopoiesis and activation in allergic humans [200].

Table 1. Major features of human mast cells and basophils

\begin{tabular}{|c|c|c|}
\hline & Basophils & Mast cells \\
\hline Origin & GMP & GMP \\
\hline $\begin{array}{l}\text { Site of } \\
\text { maturation }\end{array}$ & Bone marrow & Tissue \\
\hline Lifespan & Days to weeks & Weeks to months \\
\hline $\begin{array}{l}\text { Primary } \\
\text { location }\end{array}$ & Intravascular $(<1 \%$ of WBC) & Tissue \\
\hline Nucleus & Segmented & Ovoid \\
\hline Lipid mediators & $\mathrm{LTC}_{4}, \mathrm{LTD}_{4}, \mathrm{LTE}_{4}, \mathrm{PAF}$ & $\mathrm{LTB}_{4}, \mathrm{LTC}_{4}, \mathrm{LTD}_{4}, \mathrm{LTE}_{4}, \mathrm{PAF}, \mathrm{PGD}_{2}$ \\
\hline Granule contents & $\begin{array}{l}\text { Histamine, chondroitin sulphate, } \\
\text { protease, Charcot Leyden Crystals, } \\
\text { MBP }\end{array}$ & $\begin{array}{l}\text { Histamine, heparin and/or chondroitin } \\
\text { sulphate, protease (trypsin), tryptase }\end{array}$ \\
\hline $\begin{array}{l}\text { Differentiation } \\
\text { and chemotactic } \\
\text { factors }\end{array}$ & $\begin{array}{l}\text { IL-3,5, GM-CSF, CCL2,5,7,8,11,13 } \\
\text { CCL24,26, leptin, Flt3-L, TSLP }\end{array}$ & $\begin{array}{l}\text { IL-3,6,4,9,33, GM-CSF, NGF, SCF, } \\
\text { TSLP, CCL2,5,7,8,11,13,24,26 }\end{array}$ \\
\hline $\begin{array}{l}\text { Secreted cytokines } \\
\text { and other growth factors } \\
\text { Phenotypical markers }\end{array}$ & 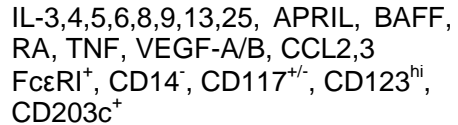 & $\begin{array}{l}\text { IL-3,5,6,8,9,13, TGF- } \beta, \text { TNF, TSLP, } \\
\text { CCL1,2,3,4,5,8,17,22 } \\
\text { FCERI }^{+}, \mathrm{CD} 14, \mathrm{CD} 117^{+}, \mathrm{CD} 203 \mathrm{c}^{+/}\end{array}$ \\
\hline
\end{tabular}

$\mathrm{APRIL}=\mathrm{a}$ proliferation-inducing ligand; $\mathrm{BAFF}=\mathrm{B}$ cell activating factor belonging to the TNF family; $\mathrm{CCL}=$ $\mathrm{CC}$ chemokine ligand; Flt3L = Flt3 ligand; GM-CSF = granulocyte-macrophage colony stimulating factor; $\mathrm{GMP}=$ granulocyte-monocyte progenitors; $\mathrm{LTB}_{4}=$ leukotriene $\mathrm{B} 4 ; \mathrm{MBP}=$ major basic protein; $\mathrm{NGF}=$ nerve growth factor; $\mathrm{PAF}=$ platelet-activating factor; $\mathrm{PGD}_{2}=$ prostaglandin $\mathrm{D} 2 ; \mathrm{SCF}=$ stem cell factor; $\mathrm{TGF}=$ transforming growth factor; TNF = tumour necrosis factor; RA = retinoic acid; TSLP = thymic stromal lymphopoietin; VEGF $=$ vaso-endothelial growth factor; $\mathrm{WBC}=$ white blood cells 


\section{Production and storage of mediators by basophils}

Basophils produce and store histamine. Upon degranulation, histamine causes symptoms such as flushing, headache and tachycardia, and is involved in the immediate allergic response as well as in anaphylaxis [207]. Basophils express histamine receptors and transporters. Intracellular histamine negatively controls its own synthesis and cytokine synthesis via the organic cation transporter 3 [208]. Besides histamine, several other lipid and protein mediators are stored and secreted by basophils, such as platelet-activating factor (PAF), which is much more potent on a molar basis than histamine [209] and leukotriene C4 (see Table 1).

Degranulation of basophils typically occurs upon IgE crosslinking after exposure to allergens. However, basophils can also be induced to degranulate by the complement factors $3 \mathrm{a}$ (C3a) and C5a, bacterial peptide fMLP, IgD and cytokines [210-212]. IL-33 alone or in combination with IL-3 enhances IgE-induced histamine release and $\mathrm{LTC}_{4}$ production, but does not induce degranulation or lipid mediator formation by itself [197]. The release of the preformed mediators causes the symptoms of immediate hypersensitivity [213].

\section{Production of cytokines}

Besides the release of preformed mediators, basophils can also produce several cytokines (Figure 2). They can rapidly produce and secrete IL-4 and IL-13 upon stimulation. This production is faster than normally expected for de novo protein synthesis and can be explained by the constitutive presence of low levels of IL-4 and IL-13 transcripts $[214,215]$. In addition, human basophils have been found to store CC chemokine ligand (CCL) 2 [216].

IL-33 synergizes strongly with IL-3 to increase IL-4 production by basophils. IL-33 belongs to the IL-1 family, is mainly expressed by fibroblasts, epithelial cells and endothelial cells and plays a key role in Th2 responses [217, 218]. Combined with $\operatorname{lgE}$ cross-linking, IL-33 also enhances histamine and IL-13 release. IL-33 also promotes mast cell- and basophil-driven inflammation and anaphylaxis, due to its ability to activate IgE-dependent and -independent effector responses [219, 220]. IL-33 induces IL9 production in human basophils, which is even more increased by simultaneous stimulation with IL-3 [221]. Several additional cytokines are produced by human basophils (see Table 1 ).

Mouse basophils not only respond to TSLP as described above, but can also produce TSLP [222]. However, it is not clear yet whether human basophils can also produce TSLP. Both mouse and human basophils produce IL-25 (or IL17-E), which has an important role in the regulation of Th2 memory cells [223]. Together with TSLP and IL33 , IL-25 can condition dendritic cells to induce a unique type of inflammatory Th2 
cells, which produce not only IL-4, IL-5 and IL-13, but also TNF- $\alpha$ instead of IL-10 [204, 224, 225]. This suggests a role for basophils in chronic allergic diseases as IL-25 and IL-25R are associated with these diseases [226].

In response to IL-3, human basophils produce retinoic acid (RA), which enhances differentiation of Th2 and Treg cells, and inhibits Th17 cell differentiation [227-229]. Human basophils produce IL-3 upon FceRI crosslinking, which acts in an autocrine fashion [198]. IL-3 induced production of amphiregulin, which is a strong Th2 stimulus and member of the epidermal growth factor family, has also been found in human basophils [230, 231]. Through FceRI crosslinking, human basophils also produce vasoendothelial growth factors $A$ and $B$ (VEGF-A and B) which are also involved in tissue remodeling [232]. These findings along with the notion that basophils produce IL-9 [221] suggest a role for basophils in tissue remodeling seen in chronic allergic inflammation [230, 231].

\section{Allergens}

Cytokines (IL-3, IL-5, IL-18, IL-33, GM-CSF, TSLP)

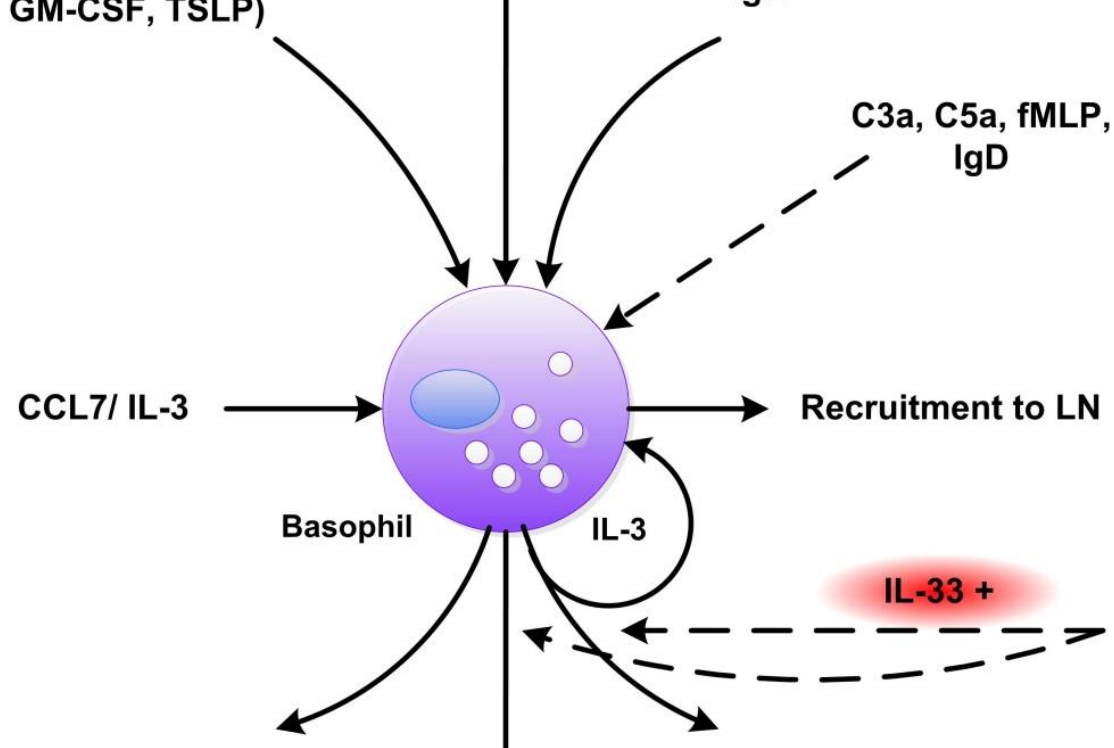

IL-4, IL-5, IL-6, IL-8, IL-9, IL-13, IL-25, RA, CCL3
IL-3, IL-4, IL-13, IL-25, VEGF-A/B, CCL3

\section{IL-4, IL-5, IL-13}

Figure 2. Activation pathways of basophils. Basophils can be activated by cytokines, allergens and $\lg \mathrm{E}$ crosslinking. Activation via one of these pathways leads to specific cytokine and chemokine responses. Cytokine responses to allergens or IgE crosslinking can be enhanced by IL-33. Basophils also respond to complement factors $\mathrm{C} 3 \mathrm{a}$ and $\mathrm{C} 5 \mathrm{a}$, bacterial peptide fMLP and $\operatorname{lgD}$ crosslinking. Furthermore, basophils can be recruited to the lymph nodes by CCL7 and IL-3. GM-CSF = granulocyte-macrophage colony stimulating factor; $L N=$ lymph node; $R A=$ retinoic acid; TSLP = thymic stromal lymphopoietin; VEGF = vasoendothelial growth factor. 
Activation of basophils may also play a role in compromising epithelial barrier function via the production of IL-4 and IL-13. An in vitro study in Calu-3 lung epithelial cells showed a disrupting effect of IL-4 and IL-13 on the epithelial barrier function and wound healing. IL-4 and IL-13 seem thus to be involved in the exacerbation seen in severe asthma patients [233].

\section{Presence of basophils at inflamed tissue sites}

Basophil infiltrates have been observed in several human allergic diseases, such as atopic dermatitis, allergic asthma and allergic rhinitis [234]. Originally, the involvement of basophils was suggested by the presence of specific mediator profiles in the late allergic responses following allergen provocation [235]. Later, specific antibodies confirmed the presence of basophils in inflamed tissue [236]. Both presence of the basophils and their state of activation indicate a role of basophils in allergic inflammation, although this has not yet been formally proven. Basophils enter tissue sites within several hours after exposure to allergens [237]. However, it is conceivable that by the time basophils enter these tissues the allergens may have been cleared already. This evidently leads to the question as to what else, other than allergen-mediated stimulation, can drive basophil activation following extravasation into tissue sites affected by allergic inflammation. Recently, it has been demonstrated that mouse basophils can be activated by IL-18 and IL-33 to release large amounts of cytokines such as IL-4, IL6, IL-9, IL-13, CCL2, CCL3, CCL4, CCL5 and GM-CSF, but not IL-17, IL-5 and interferon-y (IFN-y) [237-239].

\section{Functional role of basophils in responses to parasites and in autoimmunity}

Basophils have for long been recognized as players in Th2 immunity [188]. In addition to a role in allergy as described above, Th2 responses are important in protective immunity against parasitic infections. Basophils are involved in immunity against parasites such as the intestinal helminths Trichuris muris [201], Necator americanus [236] and Nippostrongylus brasiliensis [215, 240, 241], Schistosoma mansoni eggs [201], and ticks [190]. Basophils also protect against the microbes Moraxella catarrhalis and Haemophilus influenzae by the production of antimicrobial factors via crosslinking of membrane-bound $\operatorname{lgD}$ molecules. In addition, $\lg \mathrm{D}$ crosslinking by bacterial antigen results in support of class switching in B cells from $\lg M$ to $\lg G$ and $\lg A$ by basophilderived $B$ cell activating factor (BAFF) and a proliferation-inducing ligand (APRIL) [242]. Mouse basophils are also involved in supporting plasma cell survival [243], but these findings need to be confirmed in humans.

Besides these crucial roles in Th2 responses in allergy and parasitic infections, basophils are involved in autoimmunity. Autoimmunity is commonly described as a Th1, 
Chapter 2

Th17 and/or Treg cell-mediated response, but several autoimmune diseases are also caused by a predominant Th2 immune response. Basophils have been found to be involved in autoimmune diseases such as autoimmune urticaria [244] and bullous pemphigoid [234], and their IgD-mediated activation could imply involvement in other autoinflammatory diseases [244]. Basophils may also be involved in rheumatoid arthritis, although their role is probably redundant [245].

In systemic lupus erythematosus (SLE) Th1, Th2, Th17 and Treg cell subsets have all been described to be involved. Multiple organs seem to be affected by SLE. Kidney damage (lupus nephritis) by deposition of immune complexes formed by $\lg G$, IgM, IgA or IgE may lead to renal failure and death [246]. Rivera and colleagues [247] used a $\mathrm{Lyn}^{-/}$mouse model for lupus nephritis and showed that basophils play a crucial role in the support of autoreactive plasma cells and the secretion of autoantibodies, and the survival and differentiation of B cells, possibly via membrane-bound BAFF and IL-6 secretion. The observation of membrane-bound BAFF expression in mice is similar to what was found in human basophils, in which membrane-bound BAFF is expressed after IgD-crosslinking [242, 246, 247]. Furthermore, Lyn-/- basophils express more CD62L (L-selectin, important in recruitment to secondary lymphoid tissue), which is dependent on the presence of IL-4 and IgE. In basophils from SLE patients, the activation markers CD62L, CD203C and HLA-DR are upregulated [247]. Basophils are also detected in lymph nodes and spleens of patients, in contrast to control subjects without SLE. Thus, basophils may be held responsible for the production of autoantibodies in SLE and the perpetuation of the pre-existing loss of B cell tolerance [247].

\section{Do basophils induce Th2 type responses?}

An increasing number of papers has been published that address the role of basophils in inducing Th2 type responses. At least two major pathways have been identified by which basophils are activated to produce the Th2 signature cytokine IL-4. The IgEdependent pathway involves the binding of allergen-IgE complexes to FcERI, implicating a pre-existing immune response against the antigen, resulting in the formation of allergen-specific IgE antibodies. This raises the question which cells are involved in inducing this primary IL-4-dependent Th2 response.

Another pathway for basophilic IL-4 production is induced by the presence of cytokines such as IL-3, IL-33, or, in mice, IL-18 [248]. As discussed above IL-33 has a pronounced agonistic action with IL-3 on basophils to increase IL-4 and IL-13 release. Interestingly, IL-33 is produced, as an innate immune response, by epithelial cells upon stimulation with allergens or parasitic infection [217, 249], and is thought to be released when epithelial cells are lysed [218]. This may suggest that basophils are trig- 
gered to produce IL-4 and IL-13 in response to tissue damage, thus initiating Th2 responses in the absence of preformed lgE.

Pathogen associated molecular patterns such as proteases [248], peptidoglycan and other Toll-like receptor (TLR) ligands, but not the bacterial peptide $\mathrm{N}$-formylmethionine-leucine-phenylalanine (fMLP) or C5a [198] can also enhance the production of Th2 cytokines by basophils. Upon stimulation with Der p1, a house dust mite (HDM) protease, or $N$. americanus, human basophils produce high levels of IL-4, IL-5 and IL-13 in an IgE-independent fashion [250]. These IgE-independent activation pathways may point to an important role for basophils in providing the initial IL-4 and IL-13 needed to prime Th2 cells in response to tissue damage or infection.

However, this suggests that basophils should also be able to act as APCs. Professional APCs are very efficient in taking up, processing and presenting antigens to naïve $\mathrm{T}$ cells. They provide peptides via MHC molecules, they costimulate by molecules such as CD80 and CD86, and produce cytokines [251].

Studies in mouse models have resulted in insight in the antigen presenting processes of basophils. Mouse basophils were reported to present antigens to CD4 ${ }^{+} \mathrm{T}$ cells, and to express relevant costimulatory molecules, despite having a very low MHC-class II expression compared to DC and B cells [57]. Several studies in mouse models have even shown that basophils rather than DC are the critical APCs or at least critical providers of IL-4 for the local induction of allergen-specific Th2 type responses [201, 252, 253]. Other studies have added large doubts to these findings [254, 255].

One of the first studies that reported the necessity of basophils in inducing Th2 type responses in vivo was a study by Sokol et al (2008). The authors depleted over $90 \%$ of the basophils, but no skin or intraperitoneal mast cells by administration of the MAR-1 antibody against FceRla. They observed after papain immunization that mouse basophils are necessary to induce TSLP-dependent Th2 skewing in the lymph nodes [222]. Furthermore, they showed that basophils produce IL-4, IL-13, TSLP and CCL1 in response to papain stimulation [222]. In a follow-up study, the same group demonstrated that mouse basophils cause Th2 cell differentiation in an MHC-II-dependent and IL-4-dependent manner, both in in vitro and in in vivo experiments [252].

Further, Perrigoue et al (2009) showed that when MHC-II expression is restricted to

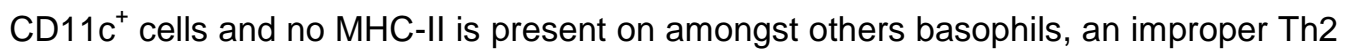
response against $T$. muris is induced. In IL-4-eGFP (4get) mice, IL-4-producing basophils have been found to respond to $T$. muris infection, expressing $\mathrm{MHC}-\mathrm{II}$ at an intermediate level [201]. Basophils can also promote the proliferation and production of IL4 by $\mathrm{CD}^{+} \mathrm{T}$ cells in vitro, which is MHC-II-dependent [201]. In another study by Yoshimoto et al (2009) basophils were the only APCs that are able to induce Th2 cells. Contrasting with other APCs, basophils pulsed with 2,4-dinitrophenyl (DNP)conjugated ovalbumin (OVA) in the presence of DNP-specific IgE antibodies have a 
Chapter 2

greater capacity to induce the proliferation of OVA-specific T cells. This can be explained by FcERI expression on basophils which mediates the effective uptake of allergen-IgE complexes leading to more efficient antigen presentation [253].

These studies clearly indicate that basophils play an important role in the induction of Th2 responses in mice. Others, however, could not reproduce these results and have found no measurable effects of basophils in mice infected with active S. mansoni or eggs after depletion of basophils by MAR-1 antibody to FceRla [256]. Instead, 70-80\% CD11 $\mathrm{C}^{+}$DC depletion in the same system as used by Sokol et al (2008) disrupted Th2 induction. This implies in contrast to the data obtained by Sokol et al (2008) that a key role for basophils in induction of the Th2 response induced by schistosome eggs may be unlikely [256].

Apart from an inducing role of basophils for Th2 responses, another model can be proposed in which DC are key APCs, but basophils provide the IL-4 and IL-13 to induce a Th2 response. HDM inhalation results in recruitment of inflammatory DC, basophils and eosinophils in a TLR-4 dependent pathway. Depletion of basophils in this model only partially reduces Th2 responses, but depletion of eosinophils has no effect on Th2 responses. Therefore, a model has been proposed whereby DC initiate and basophils amplify Th2 immunity to HDM allergen [254].

A study by Tang et al (2010) suggests that both DC and basophils are needed to generate a Th2 response. Mouse basophils immunized with endogenous or exogenous OVA plus papain are not sufficient to effectively stimulate proliferation of $\mathrm{CD} 4^{+} \mathrm{T}$ cells. Depletion of mouse basophils by injection of MAR-1 antibody does have no effect on T cell proliferation, but reduces the IL-4 production by $\mathrm{CD} 4^{+} \mathrm{T}$ cells. Furthermore, DC have been shown to have an essential role in the uptake and presentation of papain and OVA. However, DC alone are unable to produce sufficient amounts (if any) of IL-4 to induce IL-4 production in Th2 cells. Basophils alone are also unable to induce IL-4 production in Th2 cells. The combination of DC and basophils are required to induce a considerable number of IL- $4^{+}$Th2 cells. In summary, this study suggests the need of $\mathrm{DC}$ to induce $\mathrm{CD}^{+} \mathrm{T}$ cell proliferation, whereas basophils are mandatory as an accessory cell in providing IL-4 in response to papain. It has also been found that reactive oxygen species (ROS) signaling is crucial to trigger TLR4 and the subsequent production of TSLP by epithelial cells, to suppress Th1 cytokine production in DC and to induce DC-derived CCL7 production that recruits basophils via CCR3 to the lymph node [257]. However, in the studies mentioned using MAR-1 antibodies to deplete basophils, also a subset of inflammatory FcERI ${ }^{+} \mathrm{DC}$ is depleted. It is therefore not certain whether the observed impairment of Th2 induction is due to basophil or DC depletion [254].

Ohnmacht et al (2010) used transgenic Mcpt8Cre mice, which constitutively have only $10 \%$ or less basophils compared to normal mice, but have normal mast cell numbers. 
They concluded that basophils are not required in primary Th2 immunity against $N$. brasiliensis, OVA-alum and papain, and do not prime Th2 cells under these conditions. DC appear to be the key cells to induce $\mathrm{T}$ cell proliferation and differentiation upon papain challenge [258]. Min and colleagues [259] showed an additional effect of IL-3 on mouse basophils. IL-3 is required for transient recruitment of basophils to the lymph nodes after 3 to 4 days during infection with $N$. brasiliensis. Absence of IL-3 does, however, not affect the IL-4 production by $\mathrm{CD}^{+} \mathrm{T}$ cells and the Th2 immune response. They concluded therefore that basophils may be dispensable for the initiation of Th2 responses in $N$. brasiliensis infection [259]. Basophils are also found to be the major source of IL-4 during primary infection with $N$. brasiliensis, whereas IL-4 producing Th2 cells are the major source of IL-4 during secondary infection [240]. In addition, basophil migration was found to be important in mounting the Th2 response in the primary but not in the secondary infection. However, basophil-derived IL-4 is not required to support Th2 differentiation in primary nor secondary infection [240].

By imaging the interactions between basophils and CD4 ${ }^{+} \mathrm{T}$ cells, Sullivan et al (2011) showed that mouse basophils interact only briefly with $\mathrm{CD}^{+} \mathrm{T}$ cells in the lymph nodes after immunization with $S$. mansoni eggs or papain plus OVA, but they interact significantly longer with $\mathrm{CD}^{+}{ }^{+}$cells in the lung after infection with $N$. brasiliensis with or without OVA [260]. Notably, however, different immunization conditions were applied, which might have also have influenced the results.

Despite of the large number of research efforts, the precise mechanism by which basophils contribute to Th2 responses against pathogens and allergens is not entirely clear yet. It might be concluded that they only have an accessory role in which they provide IL-4 and IL-13 and act synergistically with DC. Alternatively, others clearly show that in some models basophils are the main APCs and provide IL-4 and TSLP as well (Figure 3 ). The nature of the antigen and the site where the antigen is encountered may play a crucial role in determining whether basophils are the key APC in inducing and maintaining Th2 responses, or merely are an accessory cell.

\section{Discrepancies between human and mouse basophils}

As many studies have been performed on mouse and human basophilic surface markers and functions, several phenotypical as well as functional differences have been observed. Mouse basophils can be characterized by the expression of CD11b, CD49b, CD200R3, FceRI, Thy1.2 and 2B4, and the absence of CD3, CD117, CD11c, B220, Gr1 and NK1.1 [261]. Human basophils can be characterized by the expression 


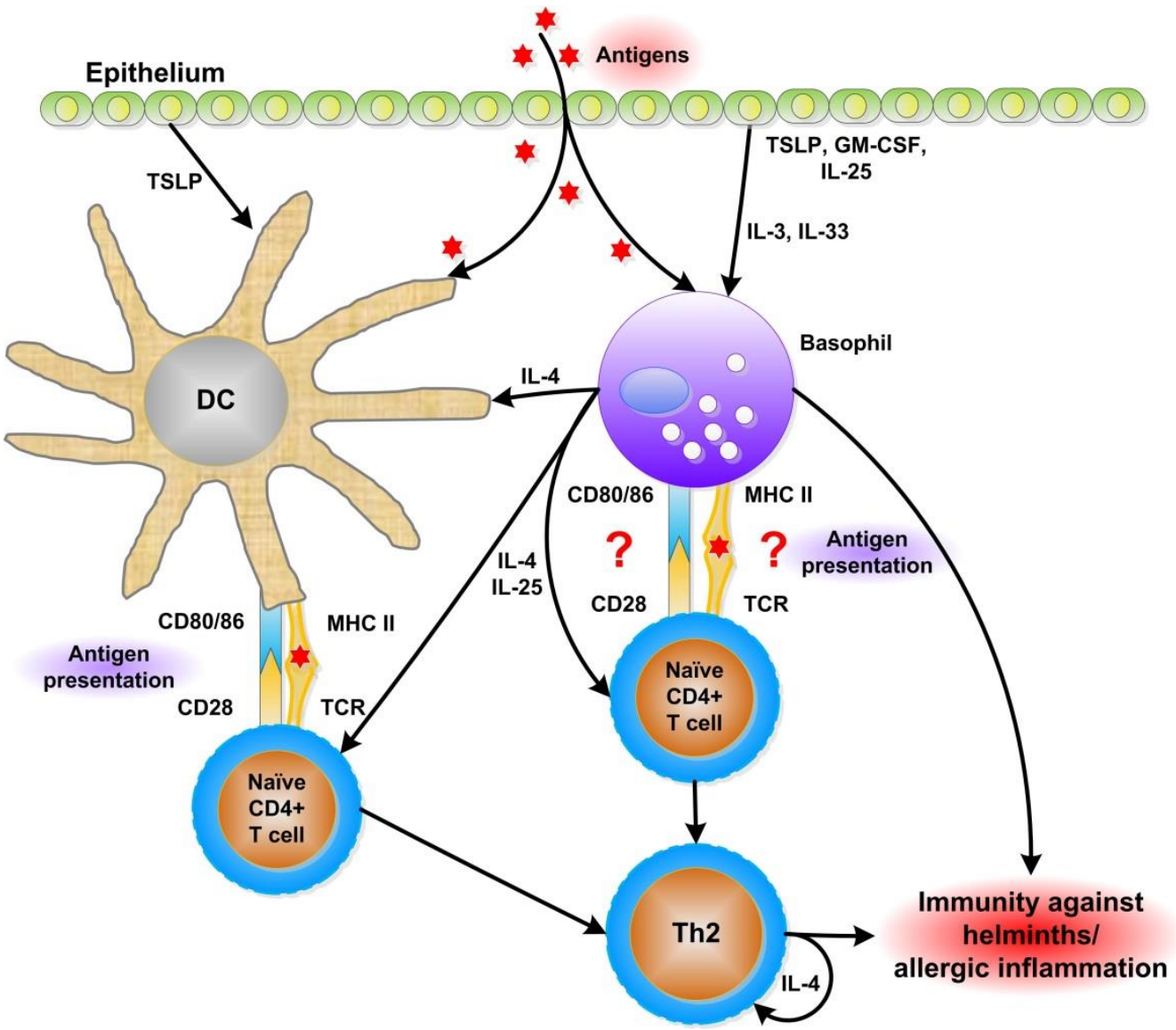

Figure 3. Integration of current knowledge on basophils. When antigen enters the body, it passes the epithelial barrier, causing tissue damage in several cases. Epithelial cells may be triggered to produce cytokines that prime basophils and DC. As a result, basophils rapidly produce IL-4, which primes naïve T helper cells to differentiate into Th2 cells. Also, IL-4 combined with TSLP activates DC to prime naïve T helper cells to differentiate into Th2 cells. Th2 cells are responsible for protective immunity against helminths and allergic inflammation. DC are known to interact with $\mathrm{T}$ helper cells to present antigen and to provide costimulation. In some mouse models, basophils do the same job. The question marks at antigen presentation by basophils in this figure underline the need for data on antigen presentation by human basophils. $D C=$ dendritic cell; GM-CSF = granulocyte-macrophage stimulating factor; TSLP = thymic stromal lymphopoietin .

of CD49b, CD123 $3^{\text {hi }}$ (IL-3R), CD192 (CCR2), CD193 (CCR3), CD203c and FcERI, and the absence of CD3, CD11C and CD14 (see Table 1). They also express several TLRs, such as TLR2 and TLR4 [198, 262]. Furthermore, they bear receptor-bound IgD on their membrane [242].

Mouse basophils induce anaphylaxis by the release of PAF via stimulation of FcyRII-III by IgG-antigen immune complexes [209]. Human basophils do express FcyRII (CD32) [263] and FcyRIIIB (CD16b) [264], but seem to lack FcyR-mediated activation due to the presence of FcyRIIB and the coupled immunoreceptor tyrosine-based inhibition motif (ITIM). In addition, FcyRIIB signaling in mouse basophils seems to differ from human basophils [265]. Furthermore, in contrast to rodents, the existence of an FcyR- 
mediated anaphylaxis in man remains controversial [266]. Therefore, it is doubtful whether human basophils are involved in FcyR-mediated anaphylaxis as observed in mice [237], although severity of human anaphylaxis is directly correlated with serum PAF levels and inversely correlated with serum PAF acetylhydrolase activity [267]. However, the contribution of PAF production by human mast cells, monocytes and macrophages is unknown. This could mean that an IgG-mediated anaphylactic pathway may exist in humans or that IgG contributes to anaphylaxis severity, but it is unclear whether human basophils or mast cells are involved in such reactions. Additional studies are needed to elucidate this question.

Another important difference between human and mouse basophils is the lack of protease-activated receptor (PAR) expression by human basophils. This could mean that the activation observed in mouse basophils by HDM [250] or papain extracts [252] is not comparable to the human situation. Additionally, IL-18 fails to activate human basophils, in contrast to mouse basophils [197]. In addition, human basophils are mostly derived from blood, whereas mouse basophils are mostly derived from bone marrow or spleen. All these differences show that caution should be applied in translating mouse research on basophils to the human situation. Some functions of basophils such as the antigen presenting function and TSLP production need to be confirmed in humans.

As discussed above, considerable functional differences have been observed between human and mouse basophils, which underlines the need of confirmation of data obtained from mouse studies in man. The role of human basophils in antigen presentation is not clear yet. There seems to be evidence that human basophils may differ from mouse basophils as they do not act as APCs. Using fluorescently labeled Bet $v 1$, Kitzmüller et al (2012) showed that human basophils efficiently bind the major birch pollen allergen Bet $v 1$ through IgE-antigen complexes, but do not internalize Bet $v 1$ and only marginally upregulate HLA-DR, and fail to induce proliferation and cytokine production in Bet v 1-specific T cells [268]. Additionally, Niederberger and colleagues [269] found that basophils of allergic patients are not capable to induce $T$ cell proliferation in secondary responses to Bet $v 1$. Various allergen-loaded APCs (DC, monocytes and macrophages), depleted of basophils, do induce T cell proliferation. Moreover, adding basophils to these APCs does not have any effect on $\mathrm{T}$ cell proliferation in allergic immune response [269]. MHC-II expression was observed in part of human basophils [270]. Research by Voskamp et al (2013) showed that the MHC- $\mathrm{II}^{+}$basophil population could be expanded in vitro by cytokines, but that this population lacked functional antigen presentation to T cells [271]. 


\section{Interaction between basophils and DC}

To determine whether basophils interact with DC, we performed cultures in which murine $\mathrm{CD} 11 \mathrm{C}^{-} \mathrm{CD} 117^{\circ} \mathrm{Fc}$ R $\mathrm{R}^{+}{ }^{+} \mathrm{BM}$-derived basophils (BMB) and BM-derived DC (BMDC) were co-cultured overnight in the presence of several stimuli (including two bacterial strains, L. plantarum WCFS1 and L. casei BL23). Activation markers on both cell types were evaluated, comparing cultures with either of the cell types to co-cultures containing both cell types. To get insight in cytokine production, cytokine levels in supernatant were measured.

Basophils enhance DC maturation in response to bacterial strains, but not in response to LPS+IL-18+IL-33 (Figure 4). LPS was used as a positive control for DC, whereas IL-18+IL-33 was used as a positive control for basophils (though these cytokines activate $\mathrm{DC}$ as well; data not shown) as described above. The frequencies of both immature $\mathrm{CD} 11 \mathrm{c}^{+} \mathrm{MHC}-\mathrm{II}^{\mathrm{lo}} \mathrm{CD} 86^{\mathrm{lo}} \mathrm{DC}(\mathrm{iDC})$ and $\mathrm{CD} 11 \mathrm{c}^{+} \mathrm{MHC}-\mathrm{Il}^{\mathrm{hi}} \mathrm{CD} 86^{\mathrm{hi}}$ mature $\mathrm{DC}(\mathrm{mDC})$ are significantly increased by adding basophils to DC cultures (Figure 4).

We then investigated the surface marker expression by DC in the presence or absence of basophils. While the presence of basophils increased the expression of CD11b and CD62L upon bacterial stimulation, it decreased CD86 expression by iDC (Figure 5). A similar effect on CD62L expression by $\mathrm{mDC}$ was observed. MHC-II expression by iDC were not significantly altered, whereas $\mathrm{mDC}$ (irrespective of the mode of stimulation) upregulated MHC-II expression when co-cultured with basophils $(p<0.01)$. CD86 expression by $\mathrm{mDC}$ was enhanced upon bacterial stimulation in the presence of basophils, as was CD62L expression. CD11b expression increased upon all tested stimuli $(p<0.05$ by one-way ANOVA).

These data show that basophils mediate DC maturation and activation, in particular upon bacterial stimulations.

iDC

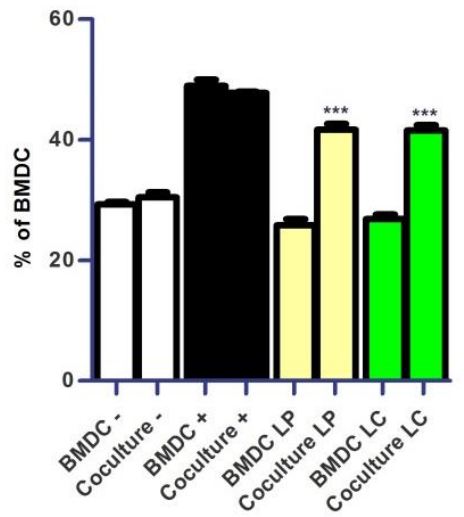

$\mathrm{mDC}$

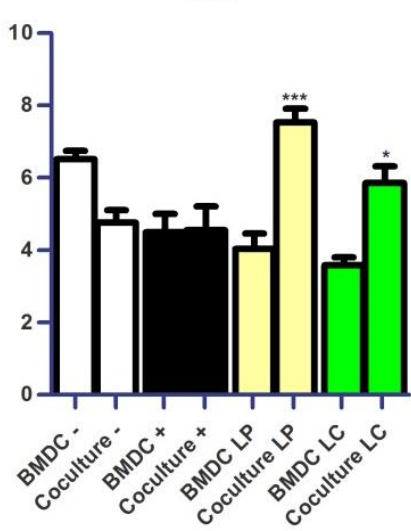

Figure 4. Basophils enhance DC maturation upon bacterial stimulation. Maturation stages of BMDC in BMDC culture and BMB-BMDC co-culture, divided in immature DC (iDC) and mature DC (mDC). - = medium control; + = LPS+IL-18+IL-33; LP $=$ L. plantarum WCFS1; LC $=$ L. casei BL23. Bars represent average values and SEM of 4-5 replicates; significant differences are indicated by asterisks. ${ }^{*}=p<0.05 ;{ }^{* * *}=p<0.001$. 
The interaction between basophils, T cells and dendritic cells
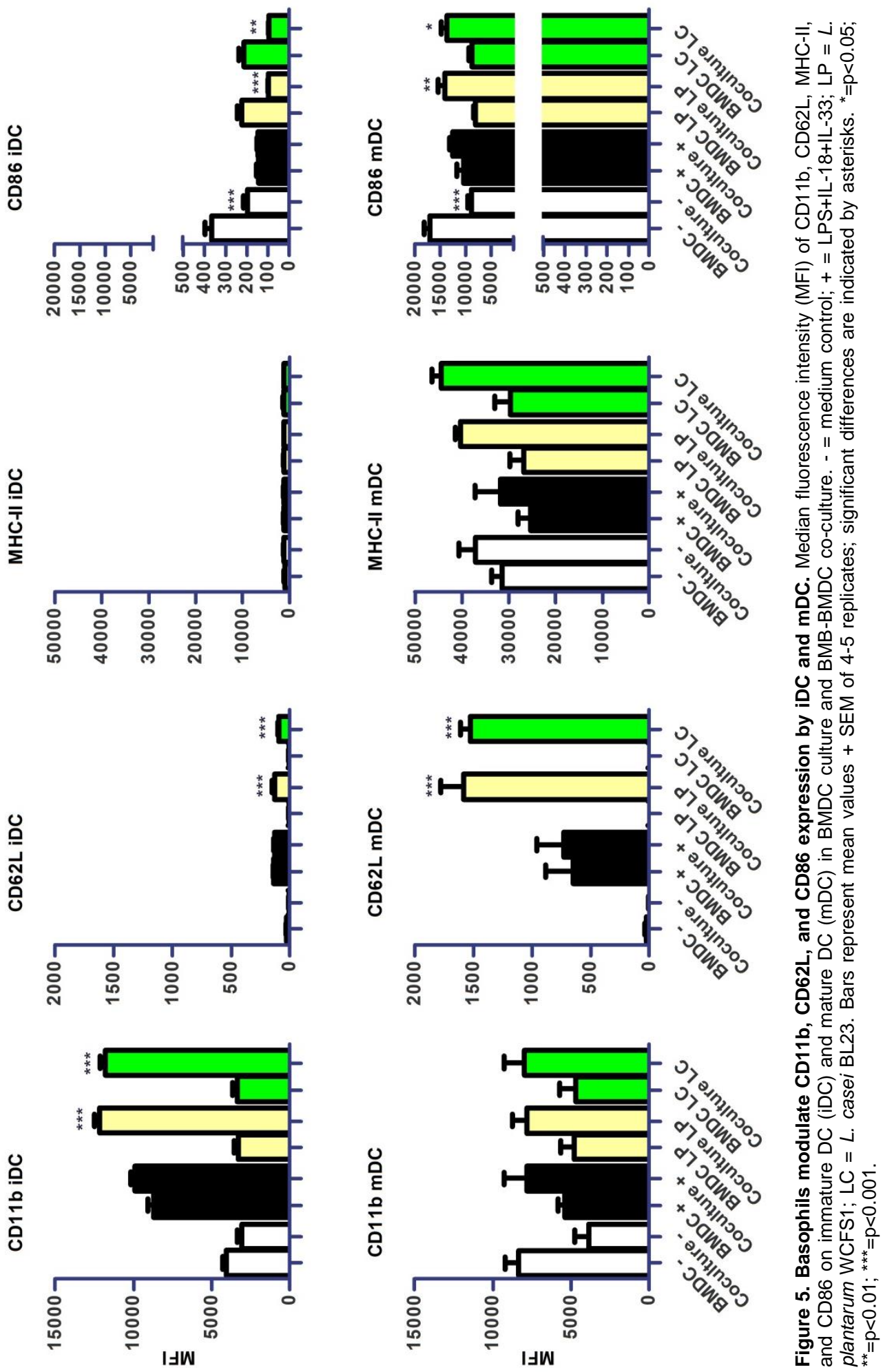
Next, we investigated whether DC influence basophil activation. We therefore compared surface marker expression of basophils, cultured alone or with DC. MHC-II and CD86 expression by basophils are very low compared to DC, and did not respond to the stimuli we applied (Figure 6). However, when co-cultured with DC, basophils down-regulated MHC-II and CD86. In addition, stimulation with bacteria or LPS+IL18+IL-33 further decreased expression of these markers. FcERla expression was reduced by the combination of co-culturing with DC and stimulation. CD11b and CD62L showed differing results per stimulus, pointing at differential activity of bacteria and LPS+IL-18+IL-33 stimulation on basophils.

These findings show that DC can instruct basophils to down-regulate activation markers. It might corroborate the proposed redundancy of basophils in antigen presentation, i.e. when the professional APC is present, basophils decrease expression of antigen-presentation and co-stimulatory molecules.

FceRI $\alpha$ BMB

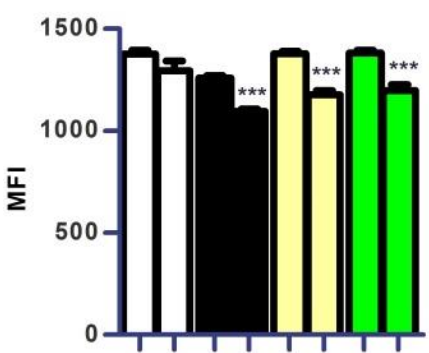

MHC-II

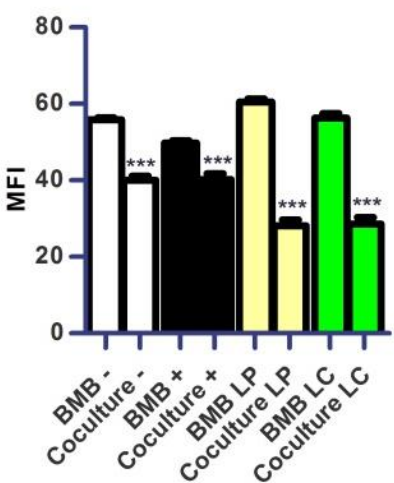

CD11b

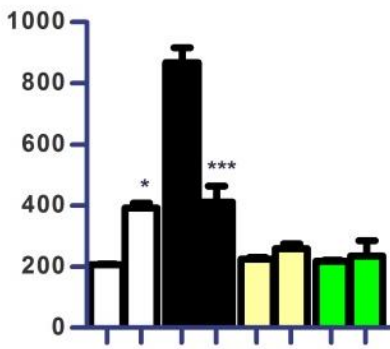

CD86

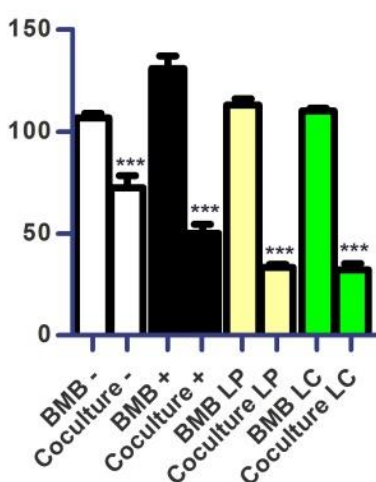

CD62L
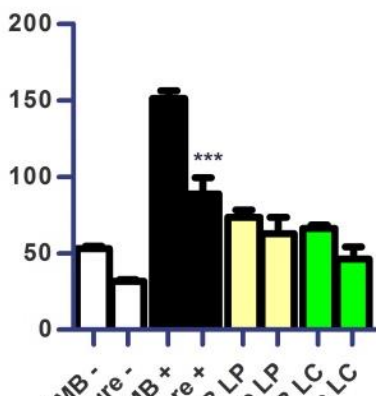

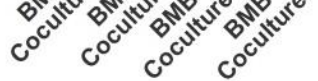

Figure 6. Surface marker expression by basophils show DC-mediated down-regulation. Median fluorescence intensity (MFI) of FceRla, CD11b, CD62L, MHC-II, and CD86 on BMB in BMB culture and BMBBMDC co-culture, as determined by flow cytometry. - = medium control; + = LPS+IL-18+IL-33. Bars represent average values and SEM of 4-5 replicates; significant differences are indicated by asterisks. ${ }^{*}=p<0.05$; ${ }^{* * *}=\mathrm{p}<0.001$.

We then determined cytokine release by basophils and DC. Comparing basophils with DC cultured alone, it is clear that DC were much more potent in producing IL-6 and 
TNF (Figure 7). Upon LPS+IL-18+IL-33 stimulation, the presence of basophils reduced IL- 6 and TNF production, whereas the presence of basophils enhanced IL-6 and TNF production upon bacterial stimulation. IL-2, IL-4, IL-10, IL-17A and IFN-ץ levels were detected in very low or non-detectable levels (data not shown).

These data show that IL- 6 and TNF release by DC is modulated by the presence or absence of basophils.

The low expression of CD62L, CD86 and MHC-II by basophils was in line with previous findings [272]. Very low levels of IL-4 were detected in basophil cultures, without visible effects of stimuli or presence of DC (data not shown). The IL-4 release observed in our cultures was much lower than reported previously [272]. An explanation for these findings could be the shorter duration of the stimulation, and the low number of plated cells.
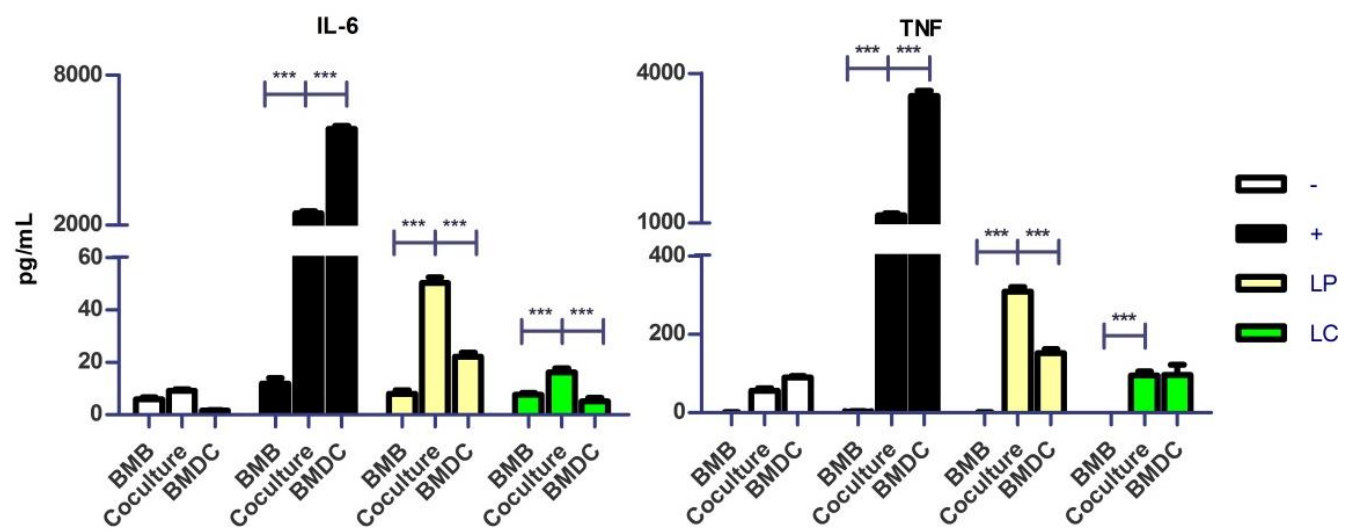

Figure 7. Basophils alter cytokine production by $\mathrm{DC}$, and vice versa. Cytokine production in supernatant of BMB culture, BMDC culture and BMB-BMDC co-culture, as determined by $\mathrm{CBA}$. - = medium control; $+=$ LPS+IL-18+IL-33. Bars represent average values and SEM of 4-5 replicates; significant differences are indicated by asterisks. ${ }^{* *}=p<0.001$.

The exact mechanisms by which basophils and DC interact with each other need to be elucidated. Cytokine-mediated interaction could play a role. Basophils are capable of producing GM-CSF, which is a growth factor for DC [273]. IL-4, in combination with GM-CSF, has also been implicated in DC maturation [274]. Additional candidates in DC maturation could be IL-25 [275-277] and TSLP [278]. On the other hand, DCderived cytokines may instruct basophils to down-regulate MHC-II and CD86. To exclude direct cell-cell interactions, basophil-derived supernatants could be added to DC (and vice versa), after which the response of either basophils or DC can be assessed. It is noteworthy that the effect of basophils on DC maturation and activation is dependent on the applied stimulus. It argues for the existence of more than one pathway by which interaction between basophils and DC occur, i.e. by different cytokines or cell-cell contact. 
Chapter 2

Collectively, our data provide the first evidence that basophils can change DC phenotype, corroborating the depicted interaction between basophils and DC as shown in Figure 3. Conversely, basophils are also modulated by DC.

\section{CONCLUSIONS}

An early Th2 skewing function and a potential role in antigen presentation by basophils was discovered recently. Some basophil functions were found to be nonredundant, unique and not shared with mast cells or other immune cells. The findings discussed in this manuscript indicate that basophils modulate the immune system by cytokine (e.g. IL-4 and IL-13) production and are players in Th2 immunity in allergies and against parasitic infections. Mouse basophils can act as APCs, but their role as APC is possibly redundant. Several findings corroborate that mouse basophils act as accessory cell to support DC in mounting a Th2 immune response, in which DC act as critical APCs and basophils provide IL-4 (Figure 3). However, most of the data presented so far is generated in mouse models. The first attempts to confirm whether human basophils exert the described basophil functions in mice, indicate a functional difference between mouse and human basophils. Future studies should focus on extrapolating important findings on mouse basophils in human basophils to make it possible to draw firm conclusions. In addition, the interaction of primary basophils with epithelium, DC and $\mathrm{CD} 4^{+} \mathrm{T}$ cells is incompletely understood. Moreover, a knowledge gap exists with regard to aging basophils, and this needs to be addressed in the near future (see Outstanding questions below). This chapter also includes experimental data on the interaction between basophils and DC, providing the first evidence that basophils might instruct DC maturation and cytokine release. These studies may yield novel therapeutic targets to improve conditions for patients suffering from allergic and autoimmune diseases in which basophils play a major role.

Outstanding questions

- Does aging affect the induction and perpetuation of Th2 responses?

- What is the effect of aging on basophil phenotype?

- Are potential APC capabilities of basophils altered with age?

- Does the accessory role of basophils alter in aging? 


\section{SUPPLEMENTAL INFORMATION}

\section{Mice}

8-12 weeks old C57BI/6J mice were kept at specified pathogen free conditions in the Wageningen University experimental animal facility. All experimental protocols have been approved by the Wageningen University committee of animal experiments. Mice were sacrificed by cervical dislocation, after which BM was harvested. BM single cell suspensions were obtained by flushing femurs, tibias and ileac bones. Cell were frozen at $-80^{\circ} \mathrm{C}$ in $90 \%$ fetal calf serum (FCS) and $10 \%$ DMSO for later use.

\section{Bacterial cultures}

Lactobacillus plantarum WCFS1 and Lactobacillus casei BL23 were grown in MRS (Merck) medium until stationary phase was reached. Viability and cfu's were checked by microscopy and by measuring optical density (OD600).

\section{Generation and purification of BMB}

BMB culture protocol was adapted from Yoshimoto et al [272]. BMB were generated by thawing BM and cultured for 11-14 days in RPMI-1640 medium containing 2 mM Lglutamin, 10\% FCS, $1 \mathrm{mM}$ penicillin/streptomycin (Sigma), $1 \mathrm{mM}$ sodium pyruvate, 50 $\mu \mathrm{M} \beta$-mercaptoethanol, 0.2\% Normocin (Invivogen) and $2 \mathrm{ng} / \mathrm{mL}$ IL-3 (Sigma). After 11-14 days, more than $50 \%$ of cells were differentiated into basophils, whereas about $10 \%$ of the cells were differentiated into dendritic cells (BMDC) or mast cells (BMMCs). At day 11-14, cells were treated with anti-CD16/32 (2.4G2, BD Biosciences), followed by incubation with CD11c-biotin (HL3, BD Biosciences) and CD117biotin (2B8, BD Biosciences). After washing, cells were stained with streptavidin-DM particles (BD Biosciences) and streptavidin-APC (Ebioscience). CD11c CD117 cells were separated using the IMagnet (BD Biosciences) and subsequently incubated with FceRla-biotin (MAR-1, Ebioscience). Cells were washed and stained with streptavidinDM particles and streptavidin-PE (Ebioscience). Dead cell exclusion was done by 7AAD staining. Live CD11 $\mathrm{C}^{-} \mathrm{CD} 117^{-} \mathrm{F} C \varepsilon R \mathrm{R}^{+}$cells (BMB) were obtained with a purity of at least 95\% (Figure S1).

\section{Generation of BMDC and BMB-BMDC co-cultures}

BMDC were generated from BM by culturing cells for 7 days in RPMI-1640 medium, $25 \mathrm{mM}$ HEPES, 10\% FCS, $1 \mathrm{mM}$ penicillin/streptomycin, $50 \mu \mathrm{M} \beta$-mercaptoethanol and $20 \mathrm{ng} / \mathrm{mL}$ rGM-CSF (BioLegend). Purified BMB and BMDC were co-cultured overnight (1:1 ratio) at $10^{5}$ cells $/ \mathrm{mL}$, in the presence of $20 \mathrm{ng} / \mathrm{mL}$ LPS (E. coli 055:B5, Sigma) + 100 ng/mL rlL-18 (MBL) + 200 ng/mL rlL-33 (PeproTech) or one of the bacterial strains L. plantarum WCFS1 or L. casei BL23 (1 cfu per BMB/BMDC). 


\section{Flow cytometry procedures}

Flow cytometry was performed according to standard procedures. Cells were incubated with CD117-PerCP-Cy5.5 (2B8, BD Biosciences), MHC-II-FITC (M5/114.152, Ebioscience), CD11b-BV421 (M1/70, BD Biosciences), CD11c-PE-Cy7 (N418, Ebioscience), CD86-APC (GL1, Ebioscience), CD62L-APC-Cy7 (MEL-14, BD Biosciences). Dead cells were excluded using 7-AAD (BD Biosciences). Refer to Figure $\mathrm{S} 2$ for flow cytometric analysis of co-cultures. IL-2, IL-4, IL-6, IFN-y, TNF, IL-17A and IL-10 secretion were measured in supernatant using the Cytometric Bead Array (CBA) Mouse Th1/Th2/Th17 Cytokine kit (BD Biosciences), according to the manufacturer's protocol. All data were acquired with a FACS Cantoll (BD Biosciences) and analyzed with FlowJo vX.0.7 (Treestar) software or FCAP Array v3.0 software (SoftFlow).
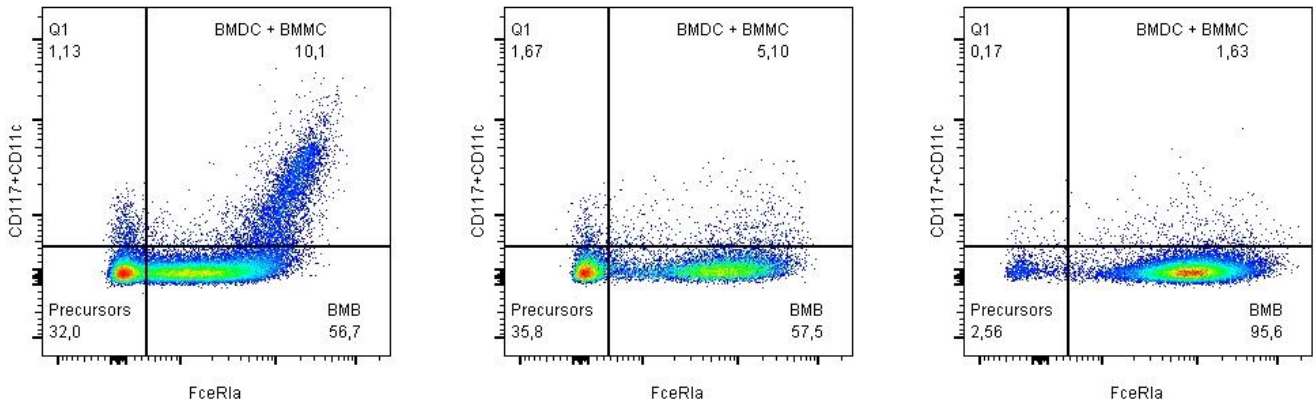

Figure S1. Identification of BMB, BMMC and BMDC in IL-3-driven cultures, and effect of purification. Representative image of BMB culture after 14 days (A). CD11C-CD117- cells after negative selection by IMagnet (B). FcERla positive selection of basophils results in more than $95 \%$ purity (C).

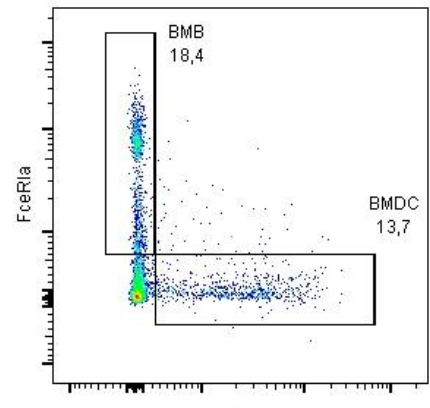

CD11c

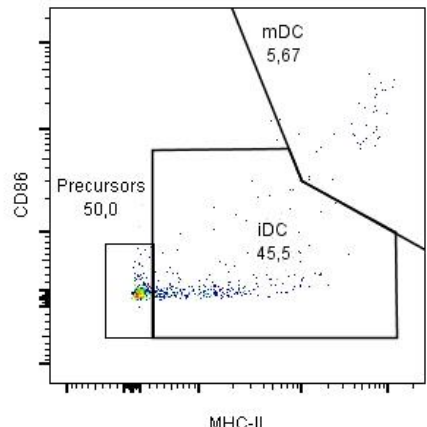

MHC-II

Figure S2. Flow cytometric analysis of BMB and BMDC co-cultures. BMB and BMDC are distinguisha-

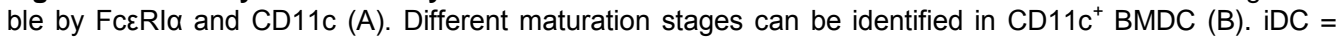
immature $\mathrm{DC} ; \mathrm{mDC}=$ mature $\mathrm{DC}$.

\section{Statistical analysis}

One-way or two-way ANOVA and subsequent Bonferroni post-tests were performed for differences in culture conditions, using GraphPad Prism v5.03 (San Diego, USA). $P$-values $<0.05$ were considered statistically significant. Significant differences are indicated by asterisks: ${ }^{*}=p<0.05 ;{ }^{* *}=p<0.01 ;{ }^{* * *}=p<0.001$. 
The interaction between basophils, T cells and dendritic cells 


\section{Chapter 3}

\section{Basophil differentiation changes during aging in mice under the influence of senescing microbiota}

Adriaan A. van Beek ${ }^{1,2}$, Floris Fransen ${ }^{2,3}$, Ben Meijer ${ }^{1}$, Paul de Vos ${ }^{2,3}$, Edward F.

$$
\mathrm{Knol}^{4,5} \text {, Huub F.J. Savelkoul }{ }^{1}
$$

${ }^{1}$ Cell Biology and Immunology Group, Wageningen University, Wageningen, the Netherlands; ${ }^{2}$ Top Institute Food and Nutrition, Wageningen, the Netherlands; ${ }^{3}$ Pathology and Medical Biology, University of Groningen, Groningen; ${ }^{4}$ Department of Immunology and ${ }^{5}$ Dermatology/Allergology, University Medical Center, Utrecht, the Netherlands 
Chapter 3

\section{ABSTRACT}

Basophils are important in Th2-mediated inflammation, which declines with age. Influence of age on basophils is poorly understood. Therefore, we studied basophil frequencies and phenotype in 4-5-month-old and 19-20-month-old mice.

With aging, basophils became more abundant in spleen. Aged basophils tended to express less CD200R3 and more CD123, and these changes were comparable and consistent in basophils from bone marrow (BM) and spleen. To investigate the role of microbiota, we transferred microbiota from young and old mice to germfree recipients. No differences between young and aged microbiota were observed on BM basophils. A tendency to lower CD11b expression was observed on splenic basophils after transferring microbiota of aged mice (compared with microbiota of young mice), with no effect on FcERla, CD200R3, CD123, TSLPR, and IL-33R expression.

To assess the changes in precursors of basophils with age, we used IL-3-driven BMderived basophil cultures. Purified FcERIa ${ }^{+} \mathrm{CD} 11 \mathrm{c}^{-} \mathrm{CD} 1177^{-}$basophils from aged mice expressed lower levels of CD11b upon several stimuli (CD200R3, IL-18+IL-33, TSLP, $\lg \mathrm{E}$ ), whereas proliferation was increased compared with basophils from young mice.

Higher frequencies of $\mathrm{IL}-4^{+}$basophils were generated from basophil precursors of aged mice (versus young mice). This seemed to be influenced by gut microbiota, as similar differences were observed in basophils derived from germfree recipients of old microbiota.

Collectively, these results indicate an important role for age determining basophil frequencies and phenotype. Furthermore, this study shows that microbiota of aged mice affect precursors of basophils, as compared with microbiota of young mice. 


\section{INTRODUCTION}

The gut contains about $10^{13}-10^{14}$ bacteria $[123,124]$. These commensal gut microbiota modulate the immune system [125] and contribute to immune homeostasis in the mucosal immune system [126]. Gut microbiota play an important modulatory role beyond mucosal immunity, for instance by changing the stem cell niche in the bone marrow (BM) [279]. Furthermore, absence of microbe-derived peptidoglycan in the circulation impairs the killing by BM neutrophils of Salmonella pneumoniae and Staphylococcus aureus [280]. In addition, in the absence of microbiota, CD123 (IL-3Ra) expression on basophil precursors was upregulated, thereby enhancing their responsiveness to IL-3 [281].

During aging the immune system develops several defects and undergoes various changes in differentiation, distribution, and activation [49]. Anti-parasitic immune responses in aged mice are impaired [282], which may indicate age-related changes in basophil function [283]. With aging, gut microbiota composition changes [107]. Basophil hematopoiesis and function are regulated by gut microbiota. Absence of gut microbiota lead to increased basophil frequencies and enhanced Th2 immune responses [281]. Histamine release and sensitivity of basophils from elderly were reported to be increased upon anti-lgE stimulation [79], but in a different study, no age-related difference was found in histamine release of human blood basophils upon anti-lgE or antiIgG4 stimulation [284]. Basophil counts were not associated with frailty or mortality in elderly women $[285,286]$. Basophil frequencies and absolute numbers decreased in blood from healthy elderly volunteers and patients suffering from Alzheimer's disease $[287,288]$. It is, however, largely unknown what effect age has on basophil differentiation and function.

Basophils are granulocytes which are involved in mounting and perpetuating Th2mediated responses [289]. Basophils are an important source of IL-4 and IL-13, which direct the immune response towards Th2 type responses [290]. After IgD crosslinking, basophils produced IL-1, IL-4 and B cell activating factor (BAFF), supporting B cell functions [242]. Basophils are the major source of IL-4 after Streptococcus pneumoniae infection, contributing to humoral memory immune responses [291]. In addition, the basophil is crucial in the pathophysiology of systemic lupus erythematosus [247], and its counts are a marker for disease activity [292]. Thus, basophils are crucial in Th2 responses.

Basophil differentiation and functions are dependent on IL-3 or TSLP [293]. Basophils can be activated in an IgE-dependent and IgE-independent manner. Regarding IgEdependent activation, FcERla crosslinking by complexes of IgE and antigen activates basophils, resulting in IL-4 and IL-13 production [213]. Basophils express IL-18R and IL-33R (ST2), and upon stimulation with IL-18 and IL-33, basophils produce IL-4, IL-6, IL-13, GM-CSF, and several chemokines [272]. This effect is further enhanced in the 
Chapter 3

presence of IL-3 [253]. CD200R3-mediated activation of basophils leads to IL-4 production in vitro, and to anaphylaxis in vivo [294].

Here we studied changes in frequency and phenotype of basophils in BM and spleen, and changes in differentiation from precursors of basophils during aging by comparing 4-month-old and 18-month-old mice. To study the influence of the aging microbiota on basophil function we studied basophil frequency and phenotype, and differentiation from precursors of basophils from young germfree recipients of microbiota of 4-monthold and 18-month-old mice.

\section{MATERIALS AND METHODS}

\section{Mice}

Young and old wild-type C57BI/6 mice were purchased from Harlan (Horst, The Netherlands). Germfree C57BI/6 mice were generated at the Central Animal Laboratory of the Radboud University Medical Center (Nijmegen, The Netherlands). Mice were kept in individually ventilated cages or sterile incubators, and were specific pathogen free (SPF). All mice had free access to feed (ssniff, rat/mouse maintenance V153X R/M-H) and water. The experiments were approved by the Animal Ethical Committee of University Medical Center of Groningen. All groups consisted of $n=10$ mice, unless otherwise mentioned.

\section{Microbiota transfers}

Feces from 4-month-old and 18-month-old female mice were freshly collected. Part of the feces was stored for microbial analysis, the remaining part was mixed with PBS. Three-month-old germfree mice were administered $200 \mu \mathrm{L}$ of $100 \mathrm{mg} / \mathrm{mL}$ fecal solution by intragastric gavage $(20 \mathrm{mg} /$ mouse). These mice were then housed in IVC for another month.

\section{Organ collection and cell suspensions}

At 4-5 months or 19-20 months of age, mice were anesthetized with isoflurane, bled, and sacrificed by cervical dislocation. Serum was collected by spinning the clotted blood, and was stored at $-80^{\circ} \mathrm{C}$ until further analysis. Mice were inspected for visible tumors, which lead to the exclusion of one aged mice. Femurs and spleen of each mouse were isolated. Single-cell suspensions of BM were obtained by flushing the femurs, whereas the spleen was cut in pieces. Cells were then passed through a cell strainer. Part of the BM cells were frozen for later use in vitro. 


\section{Flow cytometry}

Flow cytometry was performed using standard procedures. After staining for surface markers, cells were incubated with live/dead Efluor506 or Efluor520 stain (Ebioscience). Cells were then fixed using the FoxP3/Transcription Factor Staining Buffer kit (Ebioscience), with the exception of the Golgi-Stop-treated cells. They were processed using the Intracellular Fixation and Permeabilization kit (Ebioscience) to preserve intracellular cytokines. Used antibodies are listed in Table 1. Flow cytometric measurements were acquired by a FACSCanto II flow cytometry (BD Biosciences, Erembodegem, Belgium). FlowJo software vX.07 (Tree Star, San Carlos, USA) was used for data analysis.

Table 1. Used antibodies for flow cytometry and purification.

\begin{tabular}{llll}
\hline Target & Format & Clone & Company \\
\hline CD3e & FITC & $145-2 C 11$ & BD \\
CD4 & FITC & H129.19 & BD \\
CD8a & FITC & $53-6.7$ & BD \\
CD11b & BV421/FITC & M1/70 & BD \\
CD11C & Biotin/FITC & HL3 & BD \\
CD16/32 & FITC/Purified & $2.4 G 2$ & BD \\
CD19 & FITC & 1D3 & Ebioscience \\
CD45R/B220 & FITC & RA3-6B2 & BD \\
CD62L & APC-Cy7 & MEL-14 & BD \\
CD117 & Biotin & 2B8 & BD \\
& BV421 & 2B8 & BioLegend \\
& BV510 & ACK2 & BioLegend \\
CD123 & Biotin & 5B11 & BD \\
& PE & 5B11 & Ebioscience \\
CD200R3 & APC & Ba13 & BioLegend \\
FcERIa & Biotin/PE-Cy7 & MAR-1 & Ebioscience \\
IL-4 & APC & 11B11 & Ebioscience \\
IL-13 & PE-Cy7 & eBio13A & Ebioscience \\
IL-33R/ST2 & PerCP-Efluor710 & RMST2-2 & Ebioscience \\
Ki-67 & FITC & SolA15 & Ebioscience \\
Ly6C+Ly6G & FITC & RB6-8C5 & BD \\
NK1.1 & FITC & PK136 & Ebioscience \\
TER-119 & FITC & TER-119 & BD \\
TSLPR & PE & & R\&D \\
Streptavidin & APC-Efluor780 & & Ebioscience \\
\hline
\end{tabular}

\section{Basophil generation and stimulation in vitro}

BM cells were thawed, checked for viability by trypan blue, and counted. BM cells were cultures, using an optimized method that was adapted from a previously published protocol [272]. About $3.3 \times 10^{5}$ viable BM cells per $\mathrm{mL}$ culture medium were plated in 6-wells plates. Culture medium consisted of RPMI-1640 medium (Gibco, Breda, The Netherlands), $10 \%$ fetal calf serum (Gibco), $100 \mu \mathrm{g} / \mathrm{mL}$ Normocin (Invivogen, San Diego, USA), $2 \mathrm{ng} / \mathrm{mL}$ rmlL-3 (Sanquin, Amsterdam, The Netherlands), and $50 \mu \mathrm{M} \beta$ mercaptoethanol (Sigma-Aldrich, Zwijndrecht, The Netherlands). Cells were cultured for 10 days. Every 3-4 days, non-adherent cells were collected, counted, and replated. About $10^{5}$ cells were used for flow cytometry to measure proliferation and dif- 
Chapter 3

ferentiation in the cultures (see Table 1 for antibodies). Expansion of each culture was calculated by dividing the cell count by the input. After 10 days, cells were incubated with purified anti-CD16/32 and subsequently with biotinylated CD11C and CD117 (all BD Biosciences, San Jose, USA). Cells were then incubated with streptavidin-coated IMag beads (BD) and processed with the IMagnet (BD). The negative fraction was incubated with biotinylated FceRla and subsequently with streptavidin-coated IMag beads and processed with the IMagnet. The positive fraction (containing CD11c CD117 ${ }^{-} \mathrm{CER} \mathrm{Rla}^{+}$cells) were defined as BM-derived basophils (BMB), and purity typically exceeded $95 \%$ (average $>96 \%$ ). Pure BMB were resuspended to $5 \times 10^{5} / \mathrm{mL}$ and stimulated for 15 hours with culture medium (including IL-3) alone, $1 \mu \mathrm{g} / \mathrm{mL} \mathrm{rmTSLP}$ (Ebioscience, San Diego, USA), $5 \mu \mathrm{g} / \mathrm{mL}$ CD200R3 (BioLegend, San Diego, USA), 10 $\mu \mathrm{g} / \mathrm{mL} \lg E$ (Abcam, Cambridge, USA) or a combination of $50 \mathrm{ng} / \mathrm{mL} \mathrm{rmlL}-18$ (MBL International, Watertown, USA) and $100 \mathrm{ng} / \mathrm{mL} \mathrm{rmIL-33} \mathrm{(Sanquin).} \mathrm{For} \mathrm{intracellular}$ cytokine staining, cells were stimulated for 11 hours, and Golgi-Stop (BD) was added for an additional 4 hours.

\section{Statistical analysis}

All statistical analyses were performed in Prism 5.0 (GraphPad Software, San Diego, USA). For comparing two experimental conditions, unpaired Student's $T$ tests were applied (with Welch's correction if unequal variances were observed). Mann-Whitney T test was applied if no normal distribution was found with D'Agostino \& Pearson omnibus normality test. Median fluorescence intensities were tested by paired Student's T tests or Wilcoxon signed rank test (in absence of normal distribution), because all experimental groups were equally distributed at any day for acquisition. Linear regression analysis was performed to correlate $\lg E$ serum levels and basophil numbers. If testing the effect of two variables and their interaction (e.g. culture time and age), twoway ANOVA (TWA) was applied, with Bonferroni post hoc tests. Values of $p<0.05$ were considered to be statistically significant, and values between $p>0.05$ and $p<0.10$ were considered to be a trend. Significant differences are indicated by asterisks: ${ }^{*}=p<0.05 ;{ }^{* *}=p<0.01 ;{ }^{* *}=p<0.001$.

\section{RESULTS}

\section{Basophils become more abundant in the spleen during aging and display a changed phenotype}

To identify the effect of age on basophil frequencies and phenotype, we analyzed fre-

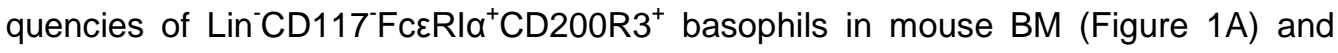
spleen (Figure 1D). By comparing young and old mice, we found that the frequencies of basophils in the BM were similar (Figure 1C), but were increased in the spleen of 


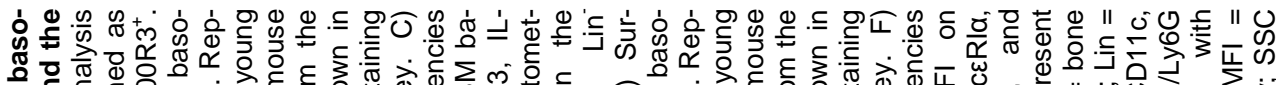

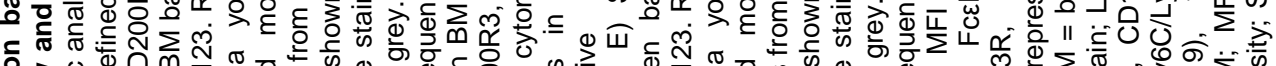

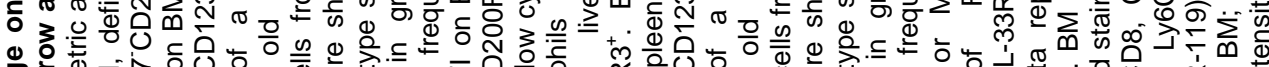

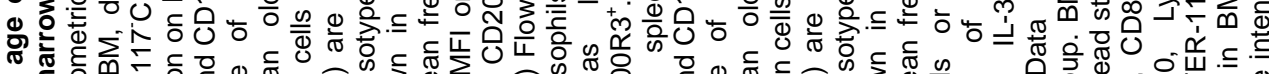

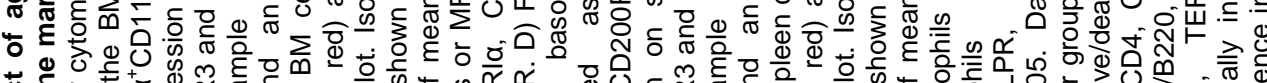

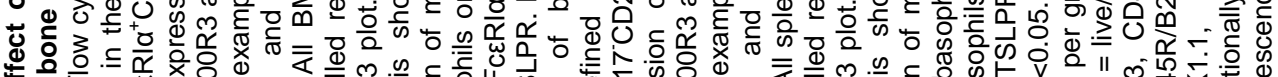

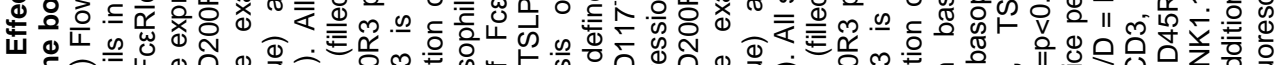

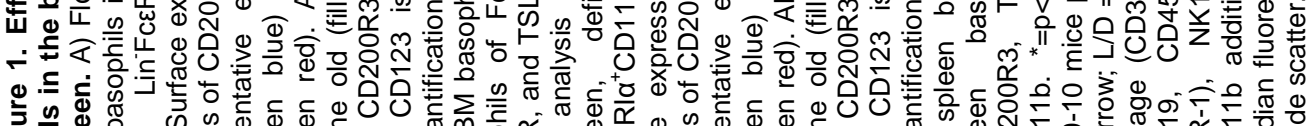

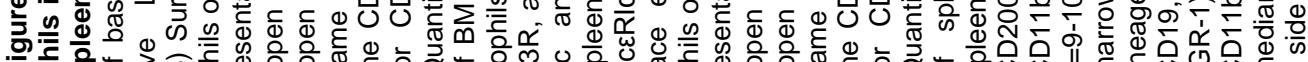

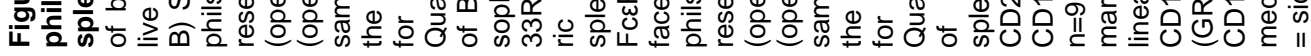

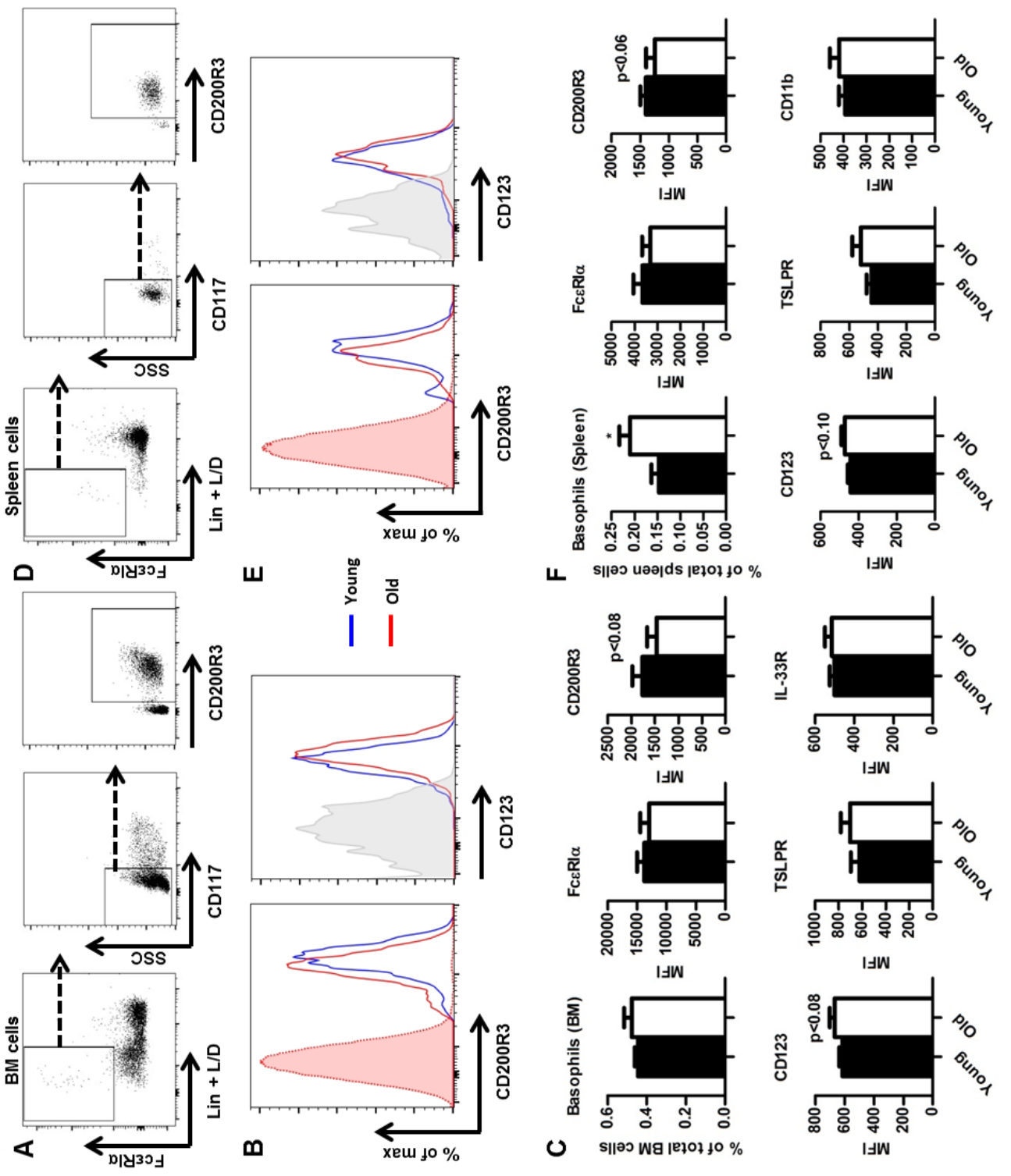


aged mice $(p<0.05$; Figure $1 F)$. The phenotype of basophils changed in both $B M$ and spleen. CD200R3 expression consistently tended to decrease on basophils in the BM $(p<0.08$; Figure 1B, 1C) and in the spleen of aged mice $(p<0.06$; Figure $1 E, 1 F)$, but CD123 expression consistently tended to be increased in aged basophils in the BM $(p<0.08)$ and spleen $(p<0.10)$. No age-related changes in FcERIa, TSLPR, CD11b, and IL-33R (Figure 1B, 1E) were observed.

\section{No difference in basophil frequencies and phenotype after microbiota transfer of young and aged mice}

Next, we questioned whether the differences with aging are caused by gut microbiota. To this end, microbiota obtained from fecal samples of 4-month-old mice or 18-monthold mice were transferred to 3-month-old germfree mice. In these young germfree mice, we found at 4 weeks after microbiota transfer no significant effects on frequency nor on phenotype of basophils (Figure 2). Both BM and spleen had similar basophil frequencies in the young or old microbiota recipients (Figure 2A, 2B). In addition, no difference in FcERla, TSLPR, CD200R3, IL-33R, and CD123 was observed between young and aged recipient-mice. The only difference we observed was in splenic basophils that tended to express less CD11b $(p<0.06$; Figure $2 B)$ in recipients of 18 -monthold microbiota.
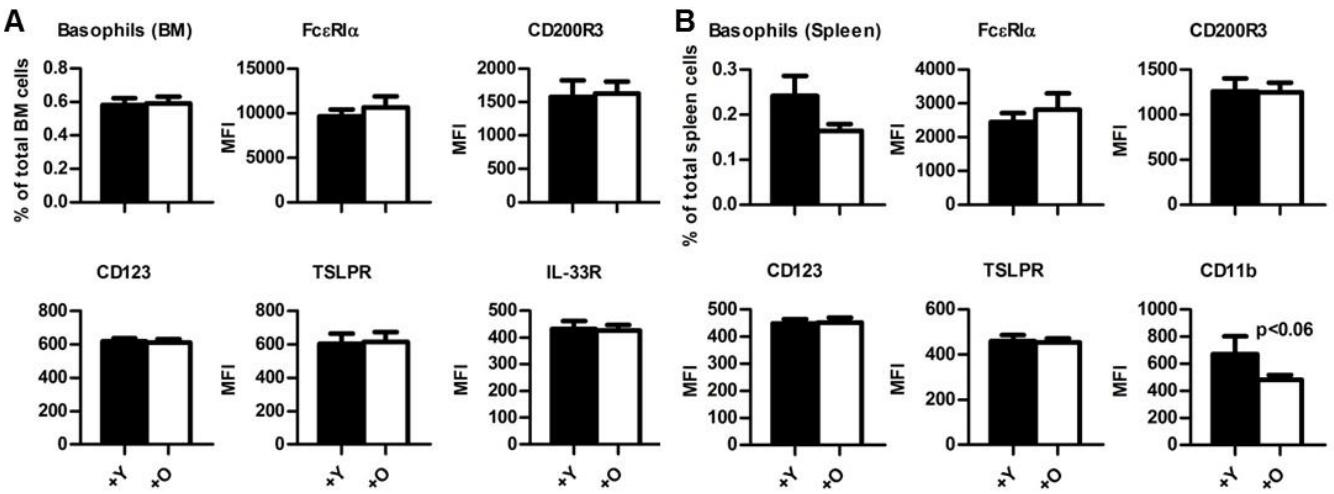

Figure 2. Effect of microbiota transfer of young and old mice to germfree mice on basophil frequencies and phenotype in the bone marrow and the spleen. A) Quantification of mean frequencies of BM basophils or median fluorescence intensity (MFI) on BM basophils of FceRla, CD200R3, IL-33R, and TSLPR. B) Quantification of mean frequencies of spleen basophils or MFI on spleen basophils of FceRla, CD200R3, TSLPR, IL-33R, and CD11b. Data represent $n=9-10$ mice per group. $B M=$ bone marrow; $O=$ microbiota derived from old mouse; $Y=$ microbiota derived from young mouse.

\section{Function of basophils is impaired in old mice in a microbiota-dependent fashion}

Although frequency and the majority of the phenotypical markers were not influenced by the age of the microbiota, we wished to exclude that other differential functional parameters of the basophils were still intact. To this end we isolated and differentiated basophils in vitro and the subsequently tested the functional response of purified basophils on several stimuli. 
Table 2. Average input, output, yield, and purity of basophils from IL-3 BMB cultures.

\begin{tabular}{|c|c|c|c|c|}
\hline Group & Input BM cells $\times 10^{6}$ & Output cultured cells $\times 10^{6}$ & Yield pure basophils $\times 10^{6}$ & Purity \% \\
\hline Young & $5.6(0.4)$ & $11.6(1.9)$ & $4.5(1.1)$ & $97(1)$ \\
\hline Old & $6.0(0.0)$ & $11.6(1.1)$ & $3.2(1.3)$ & 97 (1) \\
\hline$+Y$ & $6.0(0.0)$ & $13.7(1.6)$ & 3.7 (1.2) & $97(1)$ \\
\hline+0 & $5.6(0.4)$ & $11.0(0.7)$ & $3.4(1.0)$ & $95(2)$ \\
\hline
\end{tabular}

Data represent 5 cultures per group (with each culture derived from a different mouse). Standard error of the mean between brackets. $\mathrm{O}=$ microbiota derived from old mouse; $\mathrm{Y}=$ microbiota derived from young mouse.

Differentiation adequacy into FceRla ${ }^{+} \mathrm{CD} 117^{-}$basophils (and CD200R3 ${ }^{+}$basophils) or FceRla ${ }^{+} \mathrm{CD} 117^{+}$mast cells was determined by flow cytometry after 4,7 , and 10 days of culture (Figure 3A). No differences in expansion of the whole culture, or differentiation were observed among the experimental groups (Table 2; Figure 3B). About $98 \%$ of basophils were $\mathrm{CD}^{200 R 3^{+}}$after 10 days of culture (data not shown).

After 10 days of culturing BM cells with IL-3, we isolated the basophils (Figure 4A). Purified basophils (BMB) were overnight cultured under five different conditions: medium, IL-18+IL-33, TSLP, IgE, or CD200R3. These conditions mimic different routes of activation of basophils [289]. The five different conditions resulted in distinct basophil phenotypes. IL-18+IL-33 and CD200R3 was most potent in the induction of IL-4 and IL-13 by the basophils (Figure 4B). For Ki-67, IL-4, and IL-13, but not CD11b expression, we observed a stimulus-dependent effect (Figure 4C).

CD11b expression was decreased in BMB derived from 4-month-old mice compared with those from 18-month-old mice $(p<0.001)$. This was not microbiota-dependent, because CD11b was not altered in BMB derived from germfree recipients of old microbiota compared with recipients of young microbiota (Figure $4 \mathrm{C}$ ). We originally planned to use Ki-67 as a measure of proliferation [295], but this was not applicable as most BMB were Ki-67 $7^{+}$(Figure $4 \mathrm{~B}$ ). We therefore focused on a distinct cell population with high expression of $\mathrm{Ki}-67\left(\mathrm{Ki}-67^{++}\right)$as measure for proliferative activity. With aging, the frequency of $\mathrm{Ki}-67^{++} \mathrm{BMB}$ consistently increased under all tested conditions $(p<0.001$; Figure $4 C)$. The frequency of IL- $4^{+}$cells increased in old BMB $(p<0.05)$. This seemed to be influenced by microbiota, because similar differences were observed in BMB from recipients of old microbiota $(\mathrm{p}<0.01$; Figure $4 \mathrm{C})$. The IL- $13^{+}$frequency did not change with age, but did increase upon transfer of old versus young microbiota $(p<0.01$; Figure $4 C)$. We compared the five culture conditions in aging, and after transfer of microbiota, but found the most pronounced effects in cultures stimulated with CD200R3, IL-18+IL-33, and TSLP. 

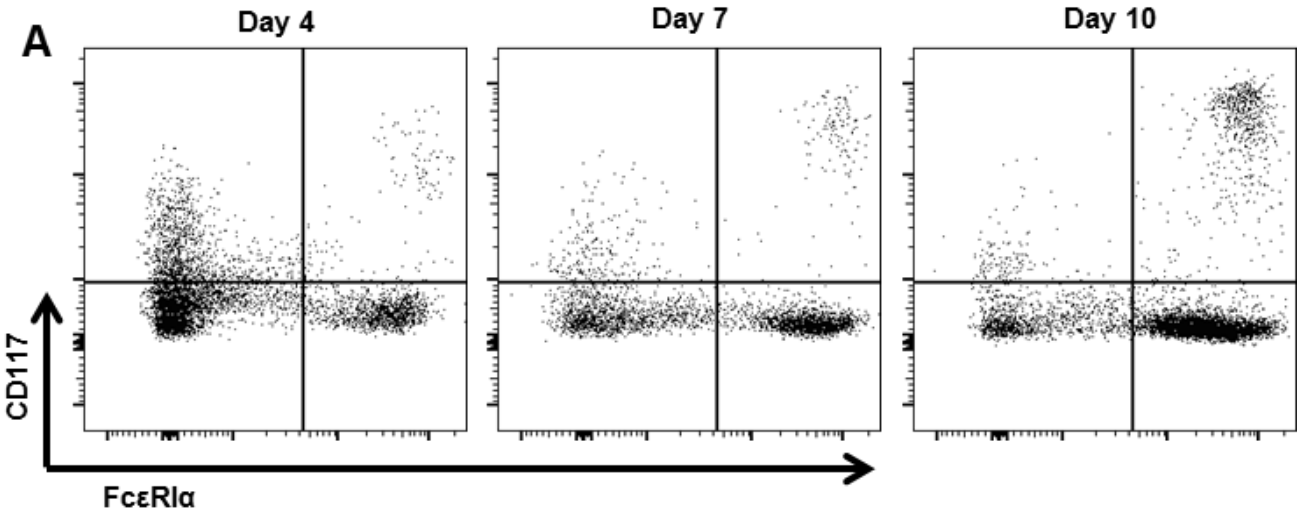

FceRla

B
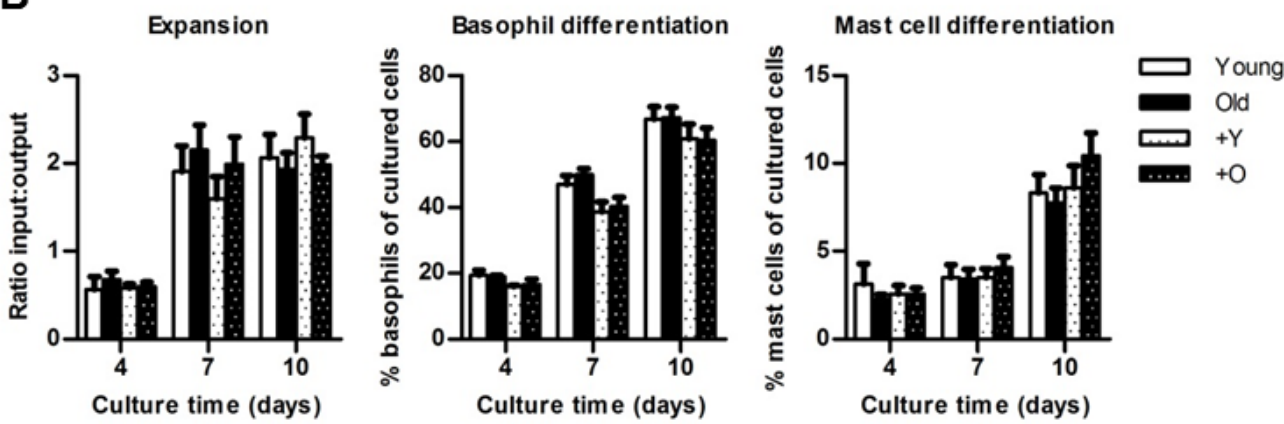

Figure 3. Effect of age and age-related microbiota on IL-3 BM cultures. A) Representative gating of IL3-driven BM culture, in which all live cells were gated for CD117 and FcERla. Basophils were defined as FcERIa $^{+} \mathrm{CD} 117{ }^{-}$and mast cells as FcERIa ${ }^{+} \mathrm{CD} 117^{+}$. Data represent $n=4$ cultures per group for day 4 and $n=5$ cultures per group for day 7 and 10 (with each culture derived from a different mouse). $\mathrm{O}=$ microbiota derived from old mouse; $Y=$ microbiota derived from young mouse.

\section{DISCUSSION}

In this study, we found that basophil frequencies and phenotype in the spleen change in mice during aging. Less effects were found in the BM. This however should not be interpreted as a suggestion that no aging effects in the BM exist, as significant effects of age were found on differentiation of basophils from precursors in the BM. Partly these effects were caused by the aging microbiota, as age-dependent changes in differentiation of basophil precursors was also observed in young germfree recipients of microbiota of 18-month-old mice.

Our report confirms age-related effects on basophils, showing for the first time that basophil phenotype changes. Intriguingly, CD123 expression by basophils from old mice consistently tended to increase. CD123 is crucial for IL-3 signaling and basophil hematopoiesis [293], and might explain the increased frequency of spleen basophils. Aged basophils showed a tendency to lower expression of CD200R3, which inhibits FcERla-mediated activation of basophils [296]. CD200R3 also activates basophils to 


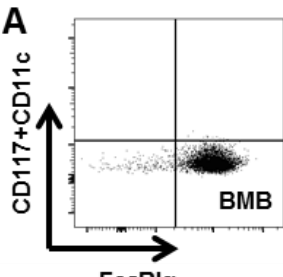

FceRla

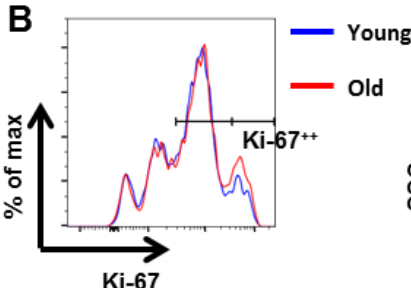

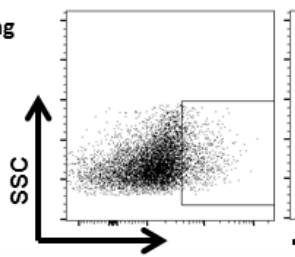

IL-4

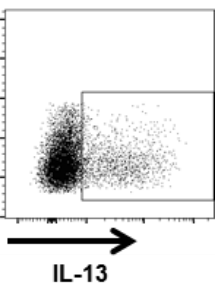

IL-13

C

CD11b

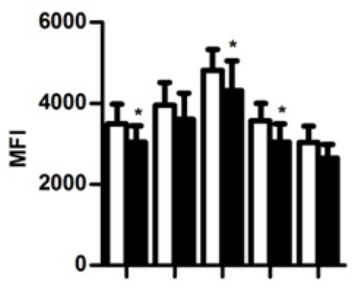

CD11b

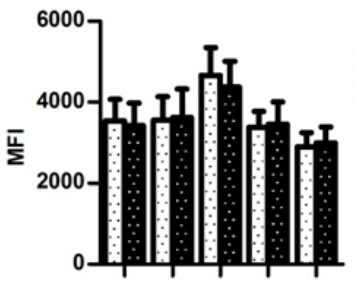

IL- $4^{+}$
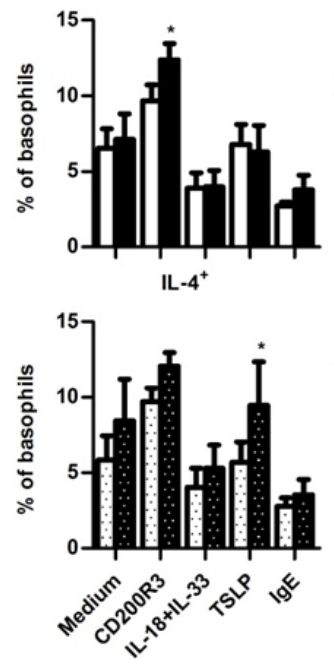

+Y

… +O

TWA

Interaction: $p=0.54$ Stimulus: $p=0.38$ Microbiota: $p=0.67$

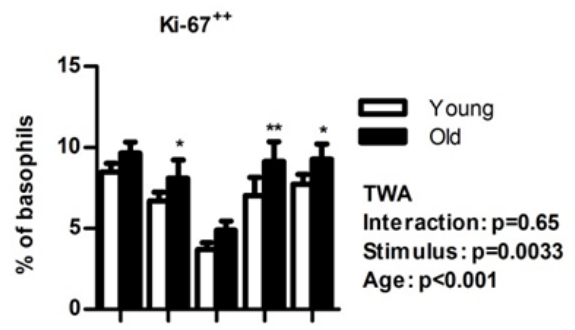

$\mathrm{Ki}-67^{++}$

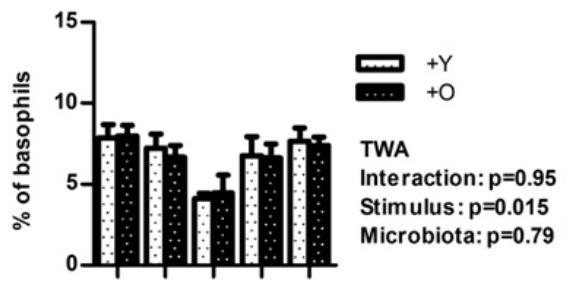

IL- $13^{+}$

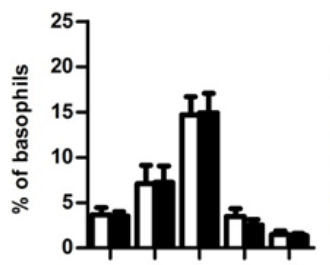

IL $-13^{+}$

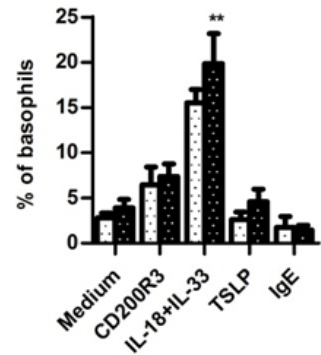

$+Y$
$+\ldots$

TWA

Stimulus: $p<0.001$

Microbiota: $p=0.0052$
Interaction: $p=0.095$

Figure 4. Effect of age and age-related microbiota on pure bone marrow-derived basophils. A) Representative example of purified BMB, defined as FcERla+CD11C-CD117-. B) Flow cytometric analysis of intracellular Ki-67, IL-4, and IL-13 staining of CD200R3-stimulated BMB. For Ki-67, a representative example of a young (blue) and an old mouse (red) is given. C) Effect of stimulation with different stimuli on expression of (extracellular) CD11b and (intracellular) Ki-67, IL-4, and IL-13. ${ }^{*}=p<0.05 ;{ }^{* *}=p<0.01$. Asterisks above bars indicate outcome of Bonferroni post hoc tests. The outcome of TWA is indicated below the legend. Data represent 5 cultures per group (with each culture derived from a different mouse). $B M B=$ bone marrow-derived basophils; $\mathrm{O}=$ microbiota derived from old mouse; TWA = two-way ANOVA; $Y=$ microbiota derived from young mouse. 
Chapter 3

produce IL-4 and to degranulate [294]. Lower CD200R3 expression by basophils from aged mice (versus basophils from young mice) might indicate that aged basophils are less readily activated [294]. Together, these age-related changes might indicate an increased sensitivity to IL-3, and at the same time an altered threshold for activation. Thus, we were able to show differences in BM and spleen basophils with age.

To gain insight into the effect of aging on the precursors of basophils, we used IL-3dependent BM cultures as a proxy (Figure 3). First, we improved the method to generate basophils by at least 70-fold compared with a recent, detailed protocol [272]. Yoshimoto et al (2012) reported using femurs and tibias of ten 9- to 12-month-old Balb/c male mice. A conservative estimation of the starting number of BM cells in their cultures is $4 \times 10^{8}$, which resulted in $20-40 \times 10^{6}$ cultured cells (culture efficiency $\leq 10 \%$ ). After purification, $1-4 \times 10^{6}$ basophils were collected (purification efficiency $\leq 10 \%$ ). Under the best conditions, the mentioned protocol ends with a $1 \%$ yield. In our hands, the culture efficiency of the improved BMB generation protocol was higher than previously reported, with each $10^{6} \mathrm{BM}$ cells generating on average $2 \times 10^{6}$ cultured cells. Taking into account the withdrawal of cells for direct assessment three times during the culture, our culture efficiency was a bit higher than $200 \%$. Our purification method, which includes dendritic cell removal, resulted in a higher numbers of pure basophils: we isolated on average $6.9 \times 10^{6}$ pure basophils per $20 \times 10^{6}$ cultured cells $(35 \%$ purification efficiency). Regardless different origins of BM (Table 2), our protocol ends with an average yield of $70 \%$. The vast difference between the yields are most likely explained by the cell density at the start of the culture. Other differences that might cause improved yield are mouse strain, fresh versus frozen BM, and the purification method. Thus, using our robust method, we were able to assess basophil function by using a few million BM cells as input. It is important to underline the importance of excluding the adherent cells during the culture and the targeted depletion of $\mathrm{CD}_{11 \mathrm{c}^{+}}$dendritic cells during the isolation of BMB. This enables to specifically look at BMB responses, without bystander effects of stromal cells or dendritic cells.

We identified additional differences between young and aged basophil precursors by using purified BMB from IL-3-dependent cultures (Figure 4). CD11b expression was decreased, whereas IL- $4^{+}$(but not IL-13 $3^{+}$) frequencies were increased in BMB from aged mice. IL- $4^{+}$basophil frequencies were particularly increased after CD200R3 stimulation, in line with previous studies [294]. BMB derived from germfree recipients receiving microbiota of aged mice (versus microbiota of young mice) also showed increased IL- $4^{+}$basophil frequencies. Thus, we found that microbiota from aged mice influence basophil precursors.

The functional implications of these findings remain to be elucidated. It is conceivable that basophils may differ in their functional response in vivo, because Hill et al (2012) showed that antibiotics under steady state conditions in vivo did not alter basophil fre- 

quencies in lymph nodes. Basophil frequencies, however, were increased after papain treatment in antibiotic-treated mice (compared with control mice) [281]. Allergic challenges or helminth infections in young versus aged mice would give insight in the functional consequences in vivo of the observed changes between young and aged basophils, and after microbiota transfers of young and aged mice.

In conclusion, our study shows age and microbiota-related changes in basophil frequencies, phenotype, differentiation, and function. Further functional in vivo studies are warranted to investigate the consequences of our findings for Th2-mediated immune responses.

\section{ACKNOWLEDGEMENTS}

We would like to thank Theo Borghuis, Mike Peters, and Stefanie Schonfeld for excellent technical assistance. 


\section{Chapter 4}

\section{Metabolic and epigenetic alterations in aging macrophages: a recipe for inflammaging?}

Adriaan A. van Beek ${ }^{1,2}$, Jan van den Bossche ${ }^{3}$, Pier G. Mastroberardino ${ }^{4}$, Menno P.J. de Winther ${ }^{3}$, Pieter J.M. Leenen ${ }^{2}$

${ }^{1}$ Cell Biology and Immunology Group, Wageningen University, De Elst 1, 6709 PG, Wageningen, The Netherlands; ' 2 Department of Immunology, Erasmus University Medical Center, Wytemaweg 80, 3015 CN, Rotterdam, The Netherlands; ${ }^{3}$ Experimental Vascular Biology, Academic Medical Center, University of Amsterdam, Meibergdreef 9, 1105 AZ, Amsterdam, The Netherlands; ${ }^{4}$ Department of Genetics, Erasmus University Medical Center, Wytemaweg 80, 3015 CN, Rotterdam, The Netherlands. 


\section{Introduction}

Aging is a complex process with impact on essentially all organs. Many diseases, including cancer, diabetes and vascular diseases, have a strong association with age, and insight into normal and abnormal aging may therefore contribute significantly to insights into disease pathogenesis. Essential characteristics of aging at the cellular level have been identified and summarized in a landmark review [297]. These features include genomic instability, epigenetic changes, telomere shortening, increased protein unfolding, mitochondrial dysfunction and dysregulated nutrient sensing. Importantly, the ability to activate cellular repair processes, such as the unfolded protein response (UPR) and autophagy decline with age [297, 298]. Cellular dysfunction, death and senescence caused by these phenomena are plausible drivers of proinflammatory cytokine production, leading to a systemic condition of chronic low-grade inflammation, also known as 'inflammaging' with increased levels of pro-inflammatory cytokines IL-1, IL-6, IL-8, TNF and C-reactive protein [299]. The main cellular sources of these mediators are not known.

Macrophages are critical regulators of processes aimed at maintaining homeostasis, and prominently contribute to inflammatory and immune responses [300], but also help maintaining metabolic stability [301]. These cells are extremely versatile to respond to environmental triggers and adapt their phenotype and function accordingly. Different stages of activation of macrophages have been identified, and so-called classically activated, or M1-polarized macrophages, and alternatively activated M2macrophages represent the ends of a full spectrum [302]. In general, M1 macrophages are catabolic, pro-inflammatory cells involved in anti-microbial host defense, while M2 macrophages are considered to be anabolic cells counteracting inflammation and stimulating tissue repair. This concept, however, is not written in stone, since also M2polarized macrophages may produce significant amounts of pro-inflammatory cytokines such as TNF, IL-1 and IL-6 upon appropriate stimulation [303] or experimental manipulation [304].

Different polarization states of macrophages are reflected in and regulated by the macrophages' metabolism [305]. Typically, M1-polarized macrophages supply their energy need from aerobic glycolysis, while M2 macrophages have higher levels of mitochondrial respiration, serving oxidative phosphorylation. Functional polarization and cellular metabolism appear to be closely intertwined as exemplified by the finding that ablating the glycolysis rate-determining enzyme PDK1 impairs inflammatory macrophage activation while enhancing M2 polarization [306].

In this review we aim to integrate current knowledge on inflammatory aspects of macrophage activation related to the aging process (Figure 1). Since macrophages are major cytokine producers and important regulators of inflammation, we approach the 
question to what extent aging-related changes in macrophages contribute to the systemic condition of inflammaging. We elaborate on how changes in autophagy, cellular metabolism, and epigenetics may contribute to or even underlie inflammaging. Insight into this matter may reveal targets for therapeutic intervention and provide directions for future research.

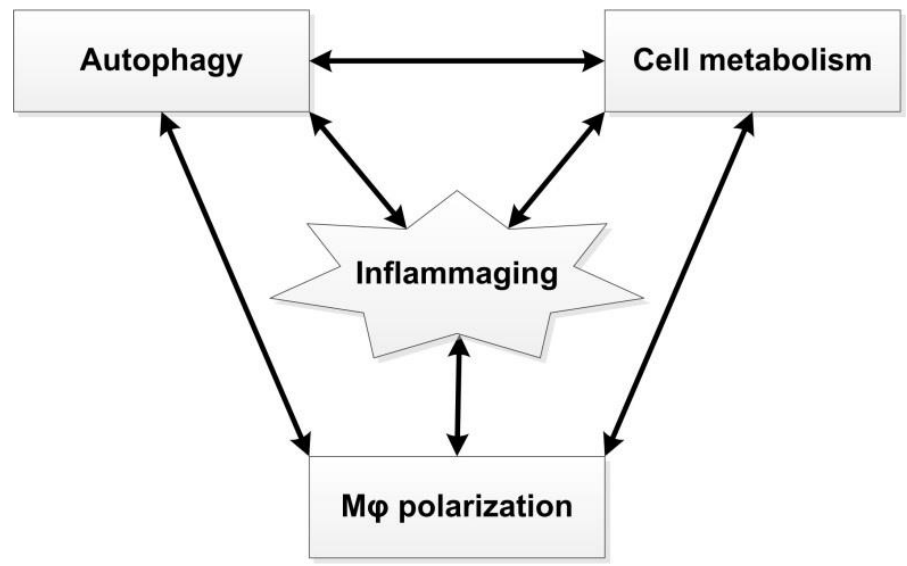

Figure 1. Aging affects autophagy, cell metabolism, and macrophage polarization. In this review we integrate current insights into changes in the cellular processes upon aging, and ask how aging macrophages contribute to the low-grade systemic inflammation known as inflammaging.

\section{Phenotypic and functional changes in aging macrophages}

Table 1 summarizes data on age-related changes in human and mouse macrophages, and it is evident that major differences are found depending on the tissue of origin. Although this may seem surprising, this observation is in line with recent notions that the local micro-environment plays a principal role in shaping the macrophage epigenetic landscape and gene expression [307, 308]. Of note, most reported age-related alterations in macrophage functions are based on ex vivo measurements, which reflect the cells' functional capacities upon challenge rather than their steady state in vivo activity.

Despite differences between distinct populations, some general messages about agerelated changes in macrophage phenotype and function may be derived. Macrophage numbers remain stable in skin, spleen, and white adipose tissue (e.g. [309]), or increase in muscle and brain (e.g. [310]). Their phagocytic capacity is unchanged or decreases with aging, which depends on the phagocytosed substances, and on the cells' origin: peritoneal macrophages show an age-related decline in phagocytosis, but this trait is unaltered in macrophages derived in vitro from bone marrow precursors (BMDM) [311, 312]. Likewise, aged splenic macrophages produce less inflammatory mediators upon stimulation compared to young macrophages, but aged and young BMDM respond similarly [313]. These results support the view that the aged microen- 
Chapter 4

vironment has a major impact on macrophage functions. However, BMDM from young and aged mice are not fully comparable in all aspects. Fei et al recently found aged BMDM to show a significantly blunted metabolic switch towards glycolysis and delayed increase in arginine metabolism upon LPS stimulation [314], indicative of imprinted changes in BM precursors, which remain demonstrable upon extensive in vitro expansion.

In general, TLR-signaling becomes less efficient with aging, although reports on agedependent alterations in TLR expression of macrophages are not uniform (e.g. [315317]). Decreased TLR signaling relates to a generally reduced ability of aged macrophages to kill micro-organisms, in conjunction with a reduced capacity to produce reactive oxygen and nitrogen species $[313,318]$. In line with this, stimulated production of pro-inflammatory cytokines IL-1 $\beta, \mathrm{IL}-6, \mathrm{IL}-12$, TNF is found to be decreased in most studies (e.g. [319-321]), although increased production of these mediators is also reported (e.g. [322, 323]). Remarkably, production of IL-10 is enhanced with aging upon stimulation of splenic macrophages and BMDM (e.g. [321, 324]. Also production of prostaglandin $(P G) E_{2}$ is generally increased in aged macrophages [325].

In sum, macrophage functions are dysregulated in aging. The causes underlying this can be divided into extrinsic factors altering the cells' environment, like changes in stromal functions, and intrinsic factors imprinted during life in mature cells and bone marrow precursors [326]. Intrinsic age-related changes are triggered by increased damage at protein, lipid and nucleic acid level, leading to senescence and organelle dysfunction [298]. We will further focus on intrinsic factors that change with age and impact significantly on normal macrophage function. Endoplasmic reticulum stress and autophagy (see Box 1) are such age-affected key processes in macrophage function.

\section{Endoplasmic reticulum stress, autophagy and inflammation}

Circumstances that cause overload of the endoplasmic reticulum (ER-) capacity, including nutrient excess, incite ER-stress and the ensuing unfolded protein response (UPR). This response is aimed at reducing this condition by diminishing protein translation, improving proper folding of newly produced proteins by increasing levels of chaperone proteins, and stimulating breakdown of misfolded proteins in cytosolic proteasomes (Box 1). Aging is associated with reduced expression of several UPR components [15]. Together with increased oxidative stress caused by mitochondrial dysfunction, this leads to increased levels of unfolded protein, thus fueling maintenance of ER-stress and consequent hampering of cellular functions.

A putative means to deal with ER-stress is to stimulate autophagy of dysfunctional cellular components, including misfolded proteins [327]. In general, autophagy is used to recycle cell material, and is also turned on during starvation to yield nutrients such 
as amino acids, fatty acids and carbohydrates [328]. However, cellular autophagy capabilities also decrease with advanced age [329].

Related to macrophages, a remarkable functional interaction exists between ERstress and macrophage polarization as ER-stress is required to generate an M2 phenotype, and suppression of ER stress causes a shift from M2 to an M1 phenotype [9]. Experimental knockdown of autophagy component Atg5 can induce M2 macrophages to produce a high levels of pro-inflammatory cytokines [304]. Moreover, several studies have shown that reduced autophagy potential, a general characteristic of aging, is related to increased production of pro-inflammatory cytokines, in particular IL-1 and IL6 [330-333]. LPS stimulation of macrophages enhances autophagy accompanied with inhibition of SIRT1 and AMPK, and induction of HIF-1a [334]. The latter conditions also activate NF-kB, thus enhancing the pro-inflammatory LPS signaling cascade. Moreover, LPS also induces ER stress in macrophages, leading to cytokine production via XBP1 [1]. Not only LPS stimulates autophagy, but also inflammatory cytokines like CCL2 and IL-6 [335]. Interestingly, these cytokines in turn stimulate M2-like polarization of macrophages [336, 337]. Together, these findings relate ER-stress and reduced autophagy to pro-inflammatory cytokine production, but in an M2- rather than classic M1-related macrophage activation profile.

The lysosomal membrane protein LAMP2a plays a role in chaperone-mediated autophagy [298], and transgenic mice with an extra copy of LAMP2a do not experience aging-associated reduction in autophagy activity [297]. Notably, LAMP2 was initially identified as the characteristic macrophage marker Mac-3 [338], likely due to the abundant presence of this protein in the lysosome-rich macrophages. It is therefore tempting to speculate that macrophages might be less sensitive to decline in autophagy potential with aging compared to other cells, but this remains to be determined.

Taken together, it can be envisaged that, also at the macrophage level, the increased ER-stress and putatively reduced autophagy potential with aging contributes to enhanced production of pro-inflammatory cytokines, and thus to systemic inflammaging. This might, however, be associated with an M2- rather than an M1- skewed profile. 


\section{BOX 1 | Concepts of ER stress and the unfolded protein response in aging}

The endoplasmic reticulum (ER) contributes to the formation of autophagosomes and is important in the proper folding of secreted proteins. A high demand for synthesis of secretory proteins is a source of stress for the ER. ER stress induces the unfolded protein response (UPR) by activating inositol-requiring protein $1 \alpha$ (IRE1 $\alpha$ ), protein kinase RNA-like ER kinase (PERK), and activating transcription factor 6 (ATF6). To restore equilibrium in the ER, information about the folding status of the ER is transduced by these three stress sensors to the cytosol and nucleus. Chronic ER stress leads to apoptosis of damaged cells [1].

Of note, the UPR is involved in many processes, such as glucose and lipid metabolism, cell differentiation, and inflammation [2]. X box-binding protein 1 (XBP1), downstream of IRE1 $\alpha$, inhibits the transcription factor forkhead box 01 (FOXO1), which is crucial in glucose homeostasis [3]. The UPR activates macroautophagy [6]. mTORC1 selectively suppresses IRE1 $\alpha$ activation [7]. TLR2 and TLR4 agonists trigger $X B P 1$ mRNA splicing in macrophages to enhance IL-6 production [8]. Elevated ER stress drives M2 macrophage polarization in a c-Jun N-terminal kinase (JNK)-dependent manner [9], and it contributes to inflammation of adipose tissue in aging [10]. Adipose tissue macrophages showed increased ER stress, accompanied with increased TNF production [10]. With aging, many components of the UPR show reduced expression and activity [15]. ER stress is implicated in metabolic and age-related diseases, such as diabetes, atherosclerosis, Alzheimer's, and Parkinson's disease [15, 16].

Taken together, ER stress and the UPR play a role in glucose metabolism, autophagy, and macrophage polarization, and are altered with aging.

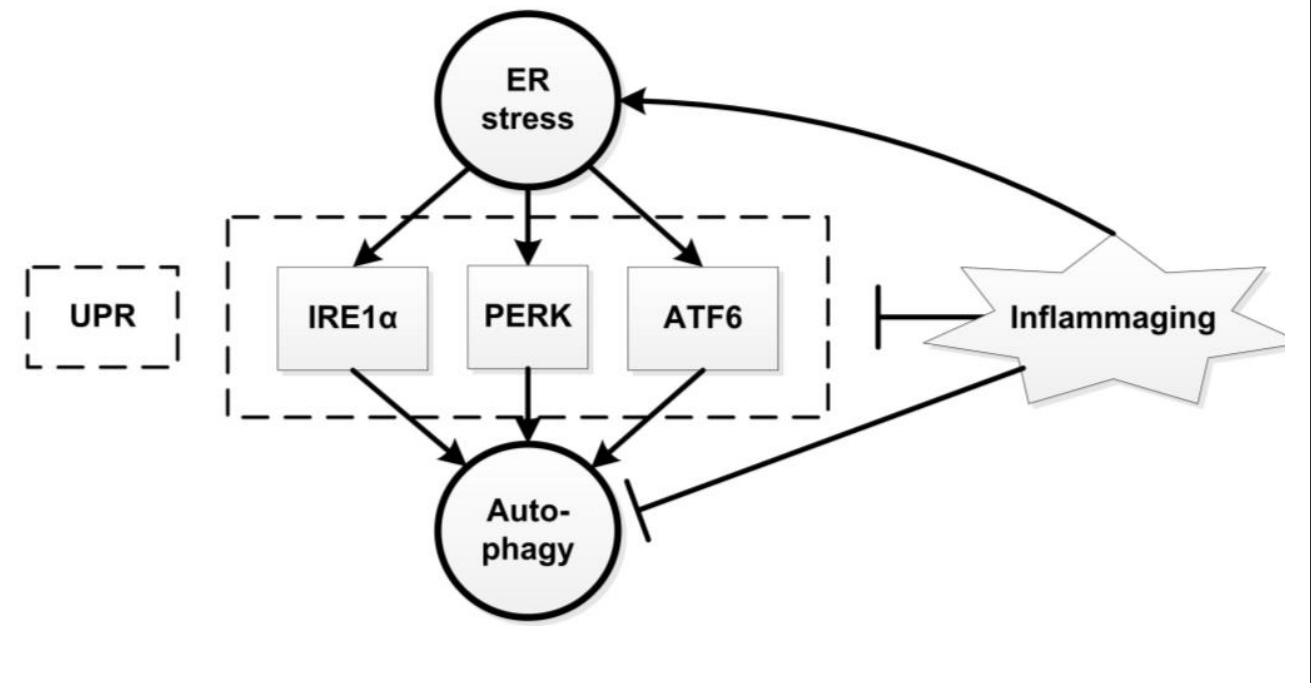




\section{Deregulated nutrient sensing and mitochondrial dysfunction in macrophage- mediated inflammaging}

Secretion of pro-inflammatory cytokines has been typically associated with M1 activation of macrophages, although also M2-polarized cells may produce these mediators, as argued above. Recent findings show that macrophage energy metabolism and inflammatory function are tightly linked [305, 339-341]. M1 activation enhances glycolysis and thereby fuels the macrophages with fast energy and biosynthetic precursors for the rapid killing of microbes. Simultaneously, glycolysis drives inflammatory responses in macrophages [342-344]. Conversely, M2-polarized cells primarily utilize mitochondrial oxidative phosphorylation (oxphos) as ATP source, and oxidative mitochondrial metabolism attenuates macrophage-mediated inflammation [306, 345, 346]. Interestingly, distinct hallmarks of aging, including decreased autophagy, deregulated nutrient sensing and mitochondrial dysfunction can increase glycolysis and suppress oxphos, thus favoring inflammatory (M1-like) activation and blunting anti-inflammatory M2 activities. In accordance, M2 macrophage numbers decrease with aging in white adipose tissue, while M1 and inflammatory macrophages remain stable or tend to increase [309]. The 'insulin and insulin-like growth factor-1 (IGF-1) signaling' (IIS) is the central pathway in nutrient signaling, and its activity declines with aging[297]. Paradoxically, further decrease of function of IIS or downstream regulators, such as AKT and mTOR, extends longevity. In macrophages, constitutively reduced ISS activity could dampen age-associated inflammatory cues since myeloid cell-specific insulin/IGF-1 receptor deficiency dampens skin inflammation and obesity-induced inflammation [347, 348]. This, however, may be only part of the operating mechanisms. The FOXO transcription factor family is another downstream target of the IIS pathway, and is inhibited by it [297]. FOXO activity is known to extend lifespan in worms and flies. While the role of FOXO in mammalian aging remains elusive, it is tempting to speculate that it facilitates inflammation in aging macrophages since FOXO1 promotes TLR4 signaling and IL-1 $\square$ production [349, 350]. Simultaneously, FOXO1 stimulates a pro-inflammatory M2 profile as well as IL-10 expression [351, 352]. Together, these studies suggest that FOXO1 promotes an inflammatory profile as well as IL-10 production in aging macrophages as a consequence of the release of FOXO inhibition with decreasing IIS signaling. 


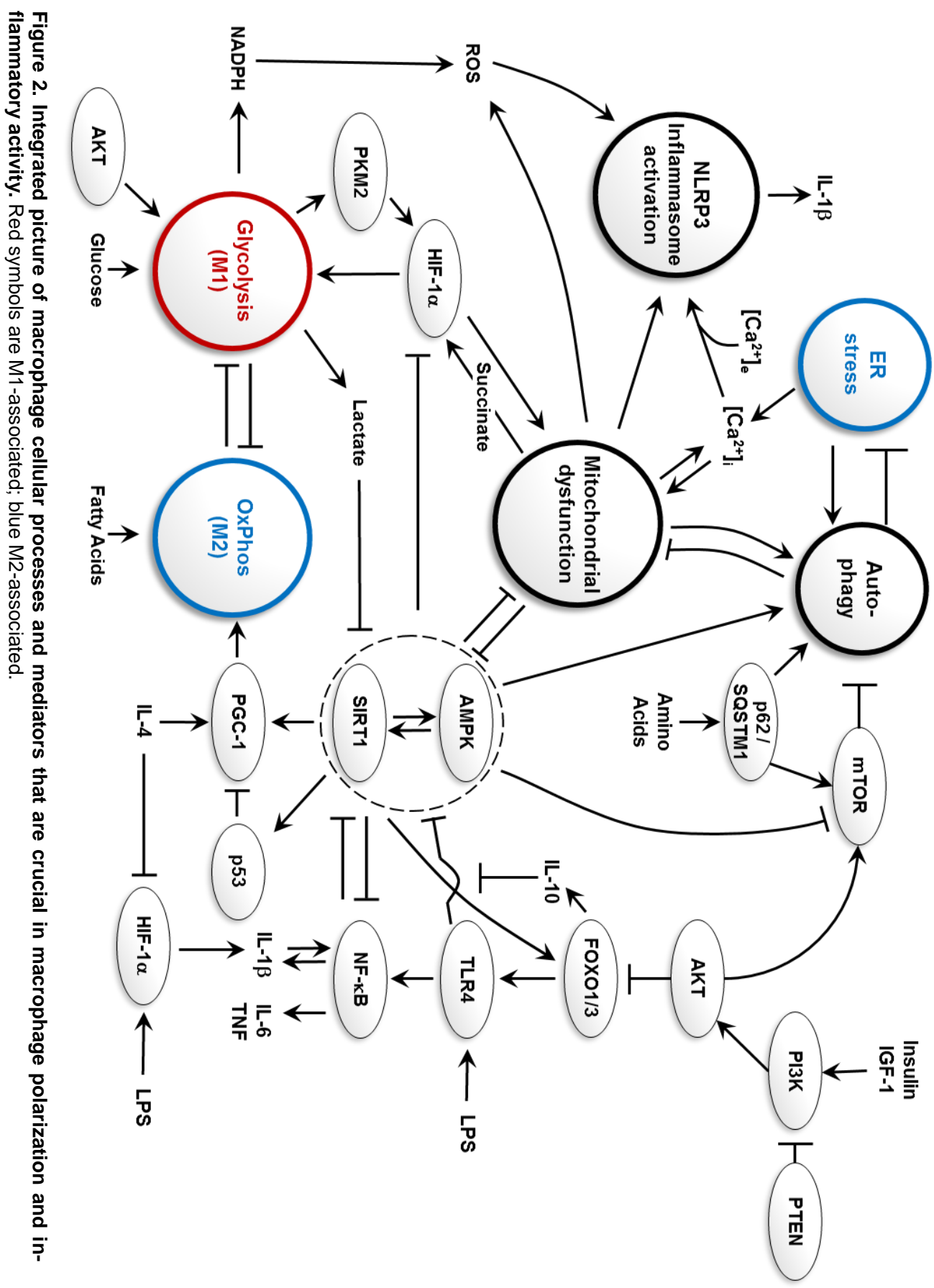


Mitochondrial dysfunction also likely promotes macrophage inflammatory activation. Several molecules (including AMPK, SIRT1 and PGC-1) with anti-aging properties promote mitochondrial function and at the same time potentiate the M2 phenotype [353-355]. AMPK activates sirtuins SIRT1 and -3, and vice versa. Subsequently,

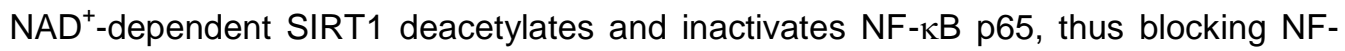
$\kappa B$-mediated inflammation [356]. In addition, SIRT1 activates PGC-1 $\beta$ and thereby promotes a switch from glycolysis to mitochondrial fatty acid oxidation that supports M2 polarization and blunts M1 activation [357]. In agreement, the M2-polarizing cytokine IL-4 promotes PGC-1 $\beta$ function, resulting in mitochondrial biogenesis, fatty acid oxidation and oxphos [346]. Interestingly, aging is associated with decreased SIRT1 transcriptional activity [358], lower nuclear $\mathrm{NAD}^{+}$levels and accumulation of HIF-1 $\alpha$ under normoxic conditions, leading to a pseudo-hypoxic state with accompanying decline in mitochondrial function [359]. HIF-1 $\alpha$ activation, as occurs in hypoxia, provides a strong pro-inflammatory stimulus for macrophages [360], thus contributing to a putative mechanistic link between elevated macrophage inflammatory status and mitochondrial dysfunction in aging.

Nevertheless, despite significant recent progress in the immunometabolism field, the molecular mechanisms by which metabolic changes affect macrophage function remain largely unexplored. Since various intermediates of energy metabolism affect histone-modifying enzymes, epigenetic mechanisms likely translate altered energy metabolism into macrophage phenotype. Interestingly, an enhanced response of monocytes to repeated exposure to Candida-derived $\beta$-glucan - termed 'trained innate immunity' - was found to be associated with the induction of enhanced aerobic glycolysis governed by a specific epigenetic repertoire [361, 362].

\section{Epigenetic regulation of macrophage activation}

Epigenetic processes, such as DNA methylation and histone methylation and acetylation, modify chromatin structure and thus play major roles in the regulation of macrophage inflammatory gene expression. During differentiation of macrophages a unique enhancer landscape is shaped that determines cell identity and collaborates with gene promoters [363]. Upon macrophage activation, signal-dependent transcription factors, such as NF- $\kappa B$, STATs, IRFs, LXR and PPARy, supplement generic and lineagedetermining transcription factors like PU.1 to mediate an inflammatory stimulusspecific transcriptional response [364].

Specific chromatin-modifying enzymes have been associated with macrophage activation and polarization, and inflammation can be modulated by targeting these [365]. The histone demethylase Jmjd3 is highly induced by bacterial products and inflammatory cytokines, and erases the repressive trimethylation mark of H3K27, thus controlling cell differentiation and inflammatory response [366, 367]. In accordance, a selec- 
tive H3K27 demethylase inhibitor decreases macrophage LPS-induced inflammatory responses [368]. Interestingly, also IL-4 stimulates Jmjd3 expression in macrophages [369], and Jmjd3 appears to be essential for normal BMDM development and subsequent M2 polarization by regulating Irf4 trimethylation, while being dispensable for M1 polarization [370]. M2 marker genes are thus epigenetically regulated by reciprocal changes in repressive H3K27 trimethylation and activating H3K4 methylation [369]. As another example, macrophage-specific deletion of the histone deacetylase HDAC3 leads to an M2-like phenotype [371], which is associated with improved lipid handling and increased plaque stability in an atherosclerosis model [372].

Besides histone modification, the DNA methylation status is key in regulating DNA accessibility as DNA hypomethylation confers accessibility to transcription factors [373]. The DNA CpG methylation status is more stable than the histone marks but also regulated dynamically [374]. Aging is characterized by general DNA hypomethylation, although site-specific hypermethylation occurs as well [375]. The DNA methylation epigenome can be interpreted as a tissue-specific measure of aging, and the rate of changes found in an individual's methylome is directly linked to alterations in transcriptional responses and may thereby influence age-related diseases [376, 377]. Alterations in epigenetic status with advancing age are directly connected to processes of cellular energy metabolism, which also change [378].

Interestingly, metabolites of common catabolic pathways supply several of the necessary co-factors that are used by epigenetic enzymes. For example, acetyl-CoA is a cofactor for histone acetyltransferases, and nuclear levels of acetyl-CoA alter the acetylation state of histones and thus provide a mechanism of epigenetic regulation [379]. Conversely, SIRT1 and other HDACs contribute to epigenetic regulation by deacetylating histones [358, 365]. Additional intracellular metabolites, like Sadenosylmethionine (SAM), $\alpha$-ketoglutarate or NAD, influence the activity of histoneand DNA-modifying enzymes and thereby potentially affect the regulation of epigenetic patterns in inflammatory cells [380].

Pathogenic triggers but also physiological and lifestyle factors can modulate epigenetic processes, thereby influencing cellular functionality. Intake of folate or ethanol, for instance, differentially influences the bioavailability of S-adenosylmethionine (SAM), a methyl donor used by histone- and DNA-methyltransferases [381]. Furthermore, carbohydrates and organo-sulphur compounds from garlic and other vegetables can stimulate butyrate generation, which acts as a general HDAC inhibitor and reduces production of pro-inflammatory cytokines [382]. Sirtuins, which can also deacetylate histones but are not inhibited by butyrate [383], are activated by polyphenols in red wine. Their activity is highly regulated by cellular NAD+/NADH levels reflecting dietary calorie levels. Interestingly, omega-3-fatty acids suppress NF- $\mathrm{kB}$ responses in macrophages by influencing activating histone methyl marks [384], which might underlie the 
observed SIRT1 activation [385]. Taken together, environmental and metabolic influences provide triggers that affect epigenetic patterns and thereby change cellular inflammatory phenotype and function upon aging.

\section{Targets to modulate inflammaging}

The mechanisms outlined above provide multiple access points to reduce inflammaging in general, and the contribution of macrophages particular (summarized in Figure 3). Dietary restriction (DR; also called calorie or caloric restriction) is well known for its life-extending effects, which are mediated by suppressing the mTOR- and IIS pathways, and enhancing autophagy [297]. DR diminishes age-dependent increase of IL-6 and TNF serum levels [386]. At high levels of DR (60\% reduction of normal food intake), however, macrophages showed dysregulated inflammatory responses [387]. A lower level of DR (40\%) led to better survival of mice after abdominal polymicrobial sepsis or endotoxemia, associated with reduced IL-6 production and WAT macrophage numbers [388]. In addition, DR might affect macrophage migration [389]. It also increases adiponectin secretion, which reduces TNF expression by macrophages and directs polarization towards M2 [389, 390]. These findings indicate that DR prevents inflammaging, also at the level of macrophages. They also underline the need for studies addressing the questions how long and at which level DR might extend life, and at the same time properly maintain macrophage function.

DR mimetics, reaching similar effects as DR without restricting energy intake, have been extensively studied. Rapamycin, which acts via mTOR, induces autophagy [391]. However, a recent study in macaques showed that rapamycin did not enhance autophagic killing of M. tuberculosis [392]. Metformin (used as treatment in type 2 diabetes, and extending mouse life span), phenformin, or berberine activate AMPK [353]. Resveratrol is a polyphenolic compound, that mimics DR in some ways. It also activates AMPK and SIRT1 (in turn activating PGC-1a) [297, 393], and it blocks LPSmediated inhibition of SIRT1 and activation of HIF-1 $\alpha$ [334]. Not surprisingly, resveratrol reduces the M1/M2 ratio in WAT after chronic intermittent hypoxia [394].

Restriction of essential amino acids like methionine or tryptophan might be another way to improve macrophage function. Methionine and tryptophan restriction are both implicated in extending lifespan in mammals [389, 395]. The tryptophan-degrading enzyme IDO expression is induced by IFN- $\gamma$, but its expression may drive M2 polarization [396]. TNF, in turn, reduces IDO expression [397]. Methionine restriction seems to decrease macrophage migration and infiltration [389]. Together, the effects of amino acid restriction on macrophage function appear to be complex and warrant further studies.

M2 macrophages produce polyamines like putrescine and spermidine, which are needed for IL-4-induced expression of several M2 markers [398]. Additionally, they 
inhibit the expression of pro-inflammatory factors in M1 macrophages. Interestingly, a probiotic strain that increases polyamine concentrations increases longevity in mice, possibly by suppression of inflammaging [165]. Along the same line, a polyamine-rich diet was shown to inhibit age-associated pathologies [399]. Based on these findings, it would be worthwhile to study whether a polyamine-rich diet in humans would extend longevity by inhibiting macrophage-mediated inflammaging.

Several other treatments have been explored, and are worthwhile to study in more detail. These include exercising, which reduces inflammaging [400], potentially by redirecting macrophage polarization into M2 direction [401]. Also exercise-mimetics like the AMPK-stimulators 5-aminoimidazole-4-carboxamide riboside (AICAR) and metformin deserve further attention [402, 403]. Counter-intuitively, these agents do not support, and may even inhibit M2 polarization of macrophages [404, 405].

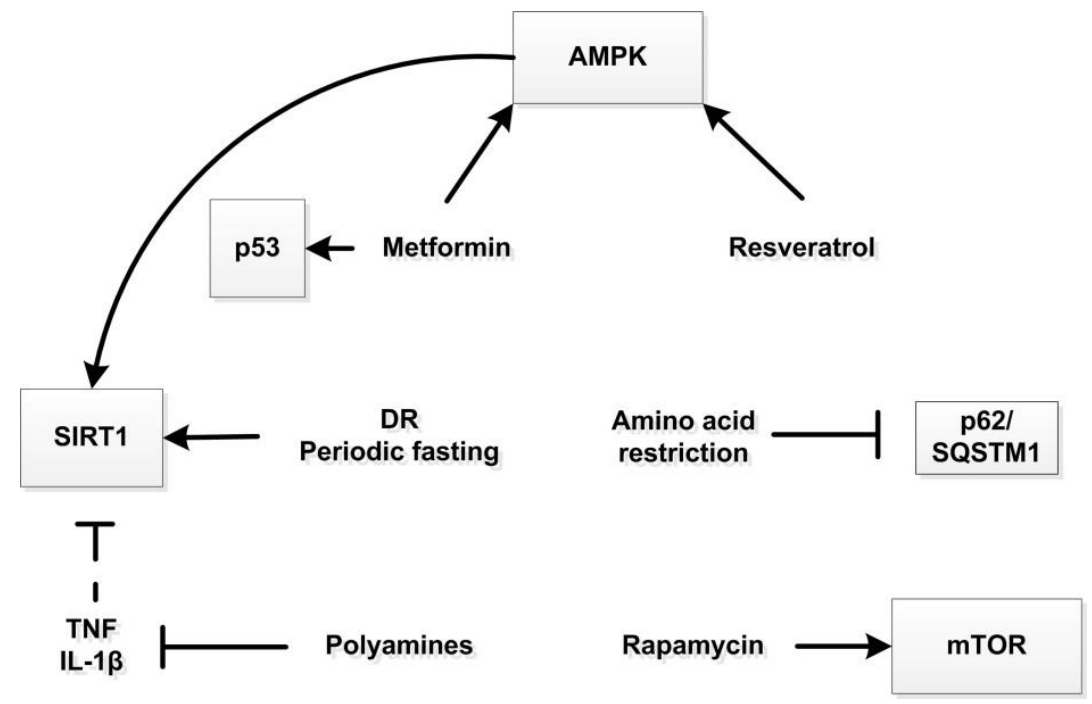

Figure 3. Putative targets in aging macrophages to modulate inflammaging.

\section{Concluding remarks}

In this review, we have aimed to address whether aging-related changes in macrophages may contribute significantly to the systemic condition of inflammaging. As major initiators, effectors and regulators of inflammatory responses, macrophages are potentially central players in this process. Taking together the aging-related changes in macrophages discussed above, and in view of the purpose of this review, two general questions come to mind: (1) Are macrophages in aging individuals the main sources of inflammatory cytokines? (2) Which functional changes in macrophages contribute to inflammaging?

The first question is most difficult to answer, as the cellular sources of increased inflammatory cytokines are essentially not known. The vast majority of studies on agingrelated phenomena reviewed here have been performed on macrophages isolated 
from their tissue environment, and especially inflammatory features have been investigated mostly after in vitro stimulation of the cells. This approach addresses the inflammatory potential of the cells rather than their activity in vivo. Macrophages are major, but not exclusive producers of inflammatory cytokines. Secretion of inflammatory cytokines is a general phenomenon of senescent cells, called the senescenceassociated secretory phenotype, and the presence of such cells increases with aging [406].

A decrease in gut barrier function with aging has been proposed as a major cause of increasing levels of TLR ligands in circulation [407]. Irrespective of changes at the cellular level in macrophages, this might already provide a major stimulating source for cytokine production by these cells and other responders. In addition, decreasing vascular function with age gives rise to increased tissue hypoxia Zhang, 2011 \#176\}, which is known as strong activator of macrophages [360]. This is mediated via HIF-1 $\alpha$ stabilization, simulating NF-kB activation. Macrophage activation by this means, however, is not easily interpreted in terms of classic M1 vs. alternative M2 polarization [360]. This stresses the notion that describing macrophage polarization in a dichotomous manner is an oversimplification, as has been confirmed by molecular profiling of macrophages activated with a wide variety of stimuli [408]. Moreover, we would advocate to separate the concepts of macrophage polarization - indicating a direction of the functional and phenotypic response to an external stimulus - and actual activation of the cells, which indicates the magnitude of the cellular response beyond its steady state activity.

Which functional changes in macrophages contribute to inflammaging? Macrophages do not escape general cellular alterations that come with aging, especially since most of the tissue-resident cells are long-lived. Thus, decreased autophagy, mitochondrial function, increased ER-stress, oxygen radical levels, etc. all trigger cellular responses in tissue macrophages that may be considered pro-inflammatory. In the sections above, the impact of these changes on the macrophages' activity have been outlined in detail. A specific functional change worthwhile elaborating here is the generally decreased phagocytic activity of aged macrophages. Since macrophages play an important role in clearance of senescent cells [409], accumulation of the latter during aging may be related to diminished macrophage function. Intriguingly, experimental removal of p16-expressing senescent cells by induction of apoptosis has been shown recently to extend the lifespan of mice [410]. It might be argued that removal of apoptotic cells is a macrophage function as well that is affected upon aging, but detailed analysis of the mechanisms by which macrophages in situ recognize and deal with senescent vs. apoptotic cells would be worthwhile investigating in the context of aging. And in general, the relative paucity of data related to in situ analysis of macrophage function during aging calls for increased efforts in this direction. 
Table 1. Age-dependent ${ }^{\star}$ alterations in human and mouse macrophages.

\begin{tabular}{|c|c|c|c|c|c|}
\hline Species & Tissue $M \varphi$ & $\#^{1,2}$ & Phenotype $^{2}$ & Functions $^{2}$ & Ref. \\
\hline \multirow[t]{5}{*}{ Human } & MDM & & $\downarrow$ TLR3 & $\uparrow$ WNV-induced TLR3, IL-6, TNF & [411] \\
\hline & & & & $\begin{array}{l}\downarrow \text { S. pneumoniae-induced IL-6, TNF, } \\
\text { P-AKT/AKT1, killing }\end{array}$ & [320] \\
\hline & & & & $\uparrow$ M. tuberculosis-induced IL-6 & [322] \\
\hline & Muscle & $\uparrow$ & & $\downarrow$ Exercise-induced IL-1 $\beta$ and IL-1RA & [412] \\
\hline & Skin & $=$ & & $\downarrow$ DTH-induced TNF & [319] \\
\hline \multirow[t]{28}{*}{ Mouse } & BMDM & & $\downarrow R X R \alpha$ & $\downarrow$ Phagocytosis of myelin fragments & [311] \\
\hline & & & & $=$ Phagocytosis of particles & [312] \\
\hline & & & & $\begin{array}{l}\uparrow \mathrm{sCD} 178 \text {-induced VEGF, } \uparrow \text { LPS-induced } \\
\mathrm{IL}-10\end{array}$ & [413] \\
\hline & & & & $\begin{array}{l}\uparrow \text { LPS-induced CCL2, } \uparrow \text { probiotic-induced } \\
\text { IL-10 }\end{array}$ & [321] \\
\hline & & & & $\downarrow$ LPS+IFN- $\gamma$-induced IL-6 & \\
\hline & & & & $\begin{array}{l}\downarrow \text { LPS-induced glycolytic shift, -arginine me- } \\
\text { tabolism }\end{array}$ & [314] \\
\hline & & & $\uparrow \mathrm{FIZZ1}$ & & [313] \\
\hline & Brain & & & $\downarrow$ Phagocytosis of myelin fragments & [311] \\
\hline & & $\uparrow^{3}$ & & & [414] \\
\hline & & & & $\uparrow\left(\right.$ LPS-induced) TNF, IL-1 $\beta$, IL-6, TGF- $\beta 1^{4}$ & [415] \\
\hline & & & $\uparrow \mathrm{MHC}-\mathrm{II}$ & $\uparrow$ LPS-induced TLR2, IDO, IL-1 $\beta$, IL-10 & [323] \\
\hline & & & & $\begin{array}{l}\downarrow \text { LPS-induced IL-4Ra and M2-related genes } \\
\text { (Arg, Ym1) }\end{array}$ & [416] \\
\hline & & & & $\downarrow \mathrm{CX}_{3} \mathrm{CR}_{1}$, LPS-induced $\mathrm{CX}_{3} \mathrm{CR}_{1}$ & [417] \\
\hline & Eye & & & $\begin{array}{l}\uparrow \text { LI-induced IL-10 } \\
\downarrow \text { LI-induced CD178, IL-12p40, TNF }\end{array}$ & [418] \\
\hline & Lung & & & $\uparrow$ LPS \pm IFN- $\gamma$-induced IL-1, IL-12, TNF, NO & [419] \\
\hline & & $=$ & $\uparrow$ TLR2 & $=M$. tuberculosis-induced cytokine response & [420] \\
\hline & & & $\uparrow$ TLR2 & $\begin{array}{l}\uparrow \text { S. pneumoniae-induced P-ERK } \\
\downarrow \text { S. pneumoniae-induced NF-KB, (P-)p38- } \\
\text { MAPK, IL-6, TNF }\end{array}$ & [316] \\
\hline & & & & $\begin{array}{l}\uparrow M . \text { tuberculosis-induced uptake, intracellu- } \\
\text { lar growth } \\
\downarrow \text { IFN- } \gamma \text {-responsiveness after M. tuberculosis } \\
\text { infection }\end{array}$ & [421] \\
\hline & & & $\begin{array}{l}\uparrow \mathrm{CD} 11 \mathrm{c}, \mathrm{MR}, \\
\mathrm{MHC}-\mathrm{II}\end{array}$ & $\begin{array}{l}\downarrow S \text {. pneumoniae-induced TRAF6 ubiquitina- } \\
\text { tion }\end{array}$ & [422] \\
\hline & & $\uparrow$ & $\uparrow \mathrm{A} 20 \downarrow \mathrm{CYLD}$ & $\uparrow$ LPS-induced P-p38 & [423] \\
\hline & Peritoneum & $=$ & & $\begin{array}{l}\downarrow \text { Phagocytosis of secondary necrotic neu- } \\
\text { trophils }\end{array}$ & [424] \\
\hline & & & & $\downarrow$ Phagocytosis of myelin fragments & [311] \\
\hline & & & & $\uparrow\left(\right.$ LPS-induced) $\mathrm{PGE}_{2}$ & [425] \\
\hline & & & & $\begin{array}{l}\uparrow \text { LPS+IFN- } \gamma \text {-induced IL-12 } \\
\downarrow \text { LPS } \pm \text { IFN- }- \text {-induced TNF, NO; HSV1 intrin- } \\
\text { sic resistance }\end{array}$ & [419] \\
\hline & & & $\downarrow$ STAT1 $1 \alpha$ & $\downarrow$ IFN- $\gamma$-induced (P-)STAT1a & [426] \\
\hline & & & & $\uparrow$ TLR4-induced and IL-1 $\beta$-induced $\mathrm{PGE}_{2}$ & [325] \\
\hline & & & & $\downarrow$ Phagocytosis of particles & [312] \\
\hline & & $\uparrow$ & $\downarrow$ TLR2 & $\begin{array}{l}=\text { Phagocytosis of } C \text {. albicans and } C \text {. albi- } \\
\text { cans-induced IL- } 10 \\
\downarrow \text { C. albicans-induced IL-1 } \beta \text {, IL-6, TNF, MIP- } \\
2\end{array}$ & [427] \\
\hline
\end{tabular}


Metabolic and epigenetic alterations in aging macrophages: a recipe for inflammaging?

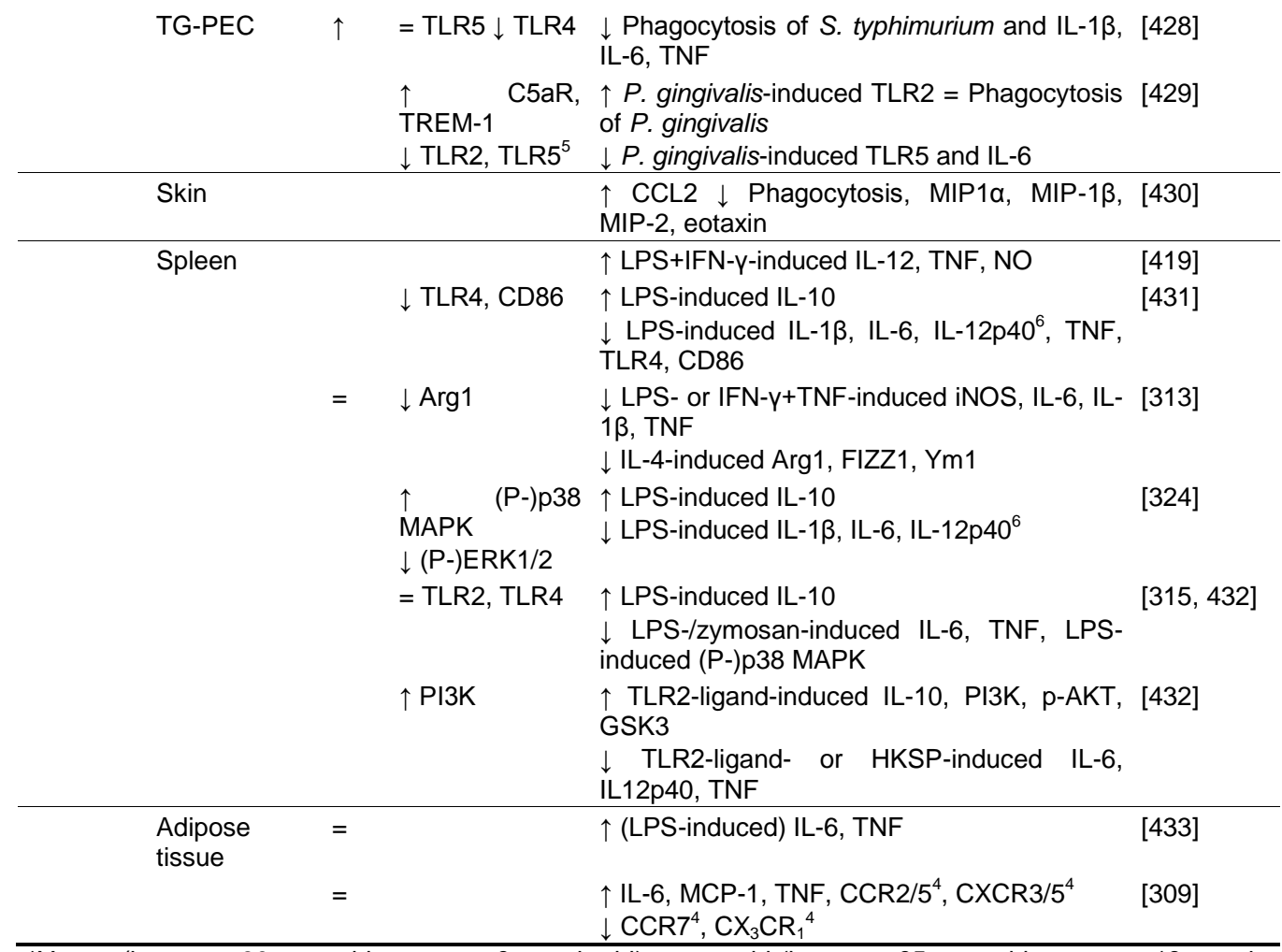

*Young (human: 30-year-old; mouse: $\leq 6$-month-old) versus old (human: $\geq 65$-year-old; mouse: $\geq 18$-monthold). ${ }^{1}$ Absolute counts. ${ }^{2}$ Increase or decrease as compared with young subjects. ${ }^{3}$ Counts in corpus callosum and cerebellum increased, non-significant increase in hippocampus, and no change in cortex. ${ }^{4} \mathrm{RNA}$ levels, no protein levels measured. ${ }^{5}$ Decreased on RNA, but not protein level. ${ }^{6}$ Personal communication. ${ }^{7}$ Trend, supported by other data. ${ }^{8}$ Three cytoskeleton components: actin, myosin, vimentin.

Abbreviations: $\mathrm{Arg}=$ arginase; $\mathrm{BM}=$ bone marrow; $\mathrm{BMDM}=$ bone marrow-derived macrophages; $\mathrm{CYLD}=$ Cylindromatosis deubiquitinase; FIZZ = found in inflammatory zone; GSK = glycogen synthase kinase; $\mathrm{HKSP}=$ heat-killed $S$. pneumoniae; $\mathrm{HO}=$ heme oxygenase; $\mathrm{HSV}=$ herpes simplex virus; $\mathrm{LI}=$ laser injury; MCP-1 = monocyte chemotactic protein 1 (CCL2); MDM = monocyte-derived macrophages; $\mathrm{MR}$ = mannose receptor; $\mathrm{P}-$ = phosphorylated; $\mathrm{PI} 3 \mathrm{~K}=$ phosphatidyl inosital 3-kinase; $\mathrm{PGE}_{2}=$ prostaglandin $\mathrm{E}_{2} ; \mathrm{TG}-\mathrm{PEC}=$ thioglycollate-elicited peritoneal exudate cells; TLR = toll-like receptor; TNF = tumor necrosis factor; TomL = tomato lectin; TREM = triggering receptor expressed on myeloid cells; VEGF = vaso-endothelial growth factor; WAT = white adipose tissue; WNV = West Nile virus . 


\title{
Chapter 5
}

\section{Interaction of mouse splenocytes and mac- rophages with bacterial strains in vitro: the effect of age in the immune response}

\begin{abstract}
A.A. van Beek ${ }^{1,2,3}$, J.A. Hoogerland ${ }^{1,2,3}$, C. Belzer ${ }^{4}$, P. de Vos ${ }^{1,5}$, W.M. de Vos ${ }^{4}$, H.F.J. Savelkoul $^{2}$, P.J.M. Leenen ${ }^{3}$

${ }^{1}$ Top Institute Food and Nutrition, Nieuwe Kanaal 9A, 6709 PA, Wageningen, The Netherlands; ${ }^{2}$ Cell Biology and Immunology Group, Wageningen University, De Elst 1, 6709 PG, Wageningen, The Netherlands; ${ }^{3}$ Department of Immunology, Erasmus University Medical Center, Wytemaweg 80, $3015 \mathrm{CN}$, Rotterdam, The Netherlands; ${ }^{4}$ Laboratory of Microbiology, Wageningen University, Dreijenplein 10, $6703 \mathrm{HB}$, Wageningen, The Netherlands; ${ }^{5}$ University of Groningen, Pathology and Medical Biology, Hanzeplein 1, 9713 GZ, Groningen, The Netherlands.
\end{abstract}

Published in Beneficial Microbes, 2016; 7(2): 275-287. 
Chapter 5

\section{ABSTRACT}

Probiotics influence the immune system, both at the local and systemic level. Recent findings suggest the relation between microbiota and the immune system alters with age. Our objective was to address direct effects of six bacterial strains on immune cells from young and aged mice: Lactobacillus plantarum WCFS1, Lactobacillus casei BL23, Lactococcus lactis MG1363, Bifidobacterium breve ATCC15700, Bifidobacterium infantis ATCC15697, and Akkermansia muciniphila ATCC BAA-835. We used splenocytes and naïve or IFN- $\gamma$-stimulated bone marrow-derived macrophages (BMDM) as responder populations. All tested bacterial strains induced phenotypic and cytokine responses in splenocytes and BMDM. Based on magnitude of the cellular inflammatory response and cytokine profiles, two subgroups of bacteria were identified, i.e. L. plantarum and $L$. casei versus $B$. breve, $B$. infantis, and $A$. muciniphila. The latter group of bacteria induced high levels of cytokines produced under inflammatory conditions, including TNF, IL-6 and IL-10. Responses to L. lactis showed features of both subgroups. In addition, we compared responses by splenocytes and BMDM derived from young mice to those of aged mice, and found that splenocytes and BMDM derived from aged mice had an increased IL-10 production and dysregulated IL-6 and TNF production compared to young immune cells. Overall, our study shows differential inflammatory responses to distinct bacterial strains, and profound age-dependent effects. These findings, moreover, support the view that immune environment importantly influences bacterial immune effects. 


\section{INTRODUCTION}

The human digestive tract is colonized by about $10^{14}$ commensal bacteria [123, 434]. Commensals digest fibers, inhibit pathogen growth, and modulate immunity [125]. These commensals thus contribute to immune homeostasis in the gut but also for instance in the bone marrow (BM) [126]. Live microorganisms that are present in foods and dietary supplements and that confer health benefits to the host are defined as probiotics [435]. Probiotics affect the course of gastrointestinal inflammatory diseases like inflammatory bowel disease (IBD) in a strain-dependent manner [161]. Inflammatory conditions also characterize the aging process. The complex process of aging is accompanied, on the one hand, by decreased immune competence, and, on the other hand, by low-grade inflammation [11, 436]. Bacterial supplementations, therefore, might be beneficial to restore immune balance in aged individuals. Indeed, beneficial effects for health in elderly subjects were shown to be induced by several LactobacilIus and Bifidobacterium strains [132, 133, 170, 171]. In addition, long-term supplementation with B. animalis subsp. lactis LKM512 extended lifespan and improved quality of life in mice [165].

Bacterial supplementations can increase intestinal epithelial barrier function and compete with colonization of pathogens [125]. One of the mechanisms underlying the crosstalk between bacteria and the host, is the presence of microbe-associated molecular patterns (MAMP) in bacteria. MAMP bind to pattern recognition receptors (PRR) present on host cells, among which mononuclear phagocytes, comprising macrophages and dendritic cells (DC), are prime responders. The activation of PRR results in cell maturation, cytokine secretion and upregulation of costimulatory molecules. By presenting antigens and secreting cytokines, macrophages and DC activate or suppress other immune cells and induce e.g. regulatory $T$ cells in order to maintain gut homeostasis [162-164]. Macrophages and DC are able to sample the gut lumen directly, in response to luminal bacteria [437-439] and thus are thought to be important regulators of gut immunity.

The direct effects of probiotics on innate immunity in aging have not been studied. Our objective, therefore, was to address direct effects of different bacterial strains on immune cells derived from young and aged mice. We used splenocytes as a complex mix of immune cells (as a 'PBMC' collection) and macrophages as a versatile responder. To test the influence of exposure of the cells to immune stimuli, we included naïve (M0) and IFN- $\gamma$-stimulated (M-IFN) macrophages [440], generated from young and aged mice. Aged splenocytes and macrophages were included to gain insight in cell-intrinsic properties that alter in aging, affecting responses to bacterial supplementation.

We selected six bacterial strains, five of which have shown probiotic activity. Lactobacillus plantarum WCFS1 is a single colony isolate of strain NCIMB8826, isolated from 
Chapter 5

human saliva [441]. Proteins derived from Lactobacillus casei BL23 have been identified to be responsible for its beneficial health effects [442, 443]. Lactococcus lactis MG1363 is originating from cheese starter derivatives and is studied extensively for its use as prototype for bioactive molecule delivery in the gut [444, 445]. Bifidobacterium breve ATCC15700 is not widely studied. Other B. breve subspecies, however, induce anti-inflammatory responses after allergic sensitization [446]. Bifidobacterium infantis ATCC15697 increases epithelial barrier function in the gut, and attenuates induced colitis in mice [447]. Presence of Akkermansia muciniphila has been inversely correlated to acute appendicitis [448]. This bacterium is known as an intestinal mucus degrader and it is abundant in the human digestive tract $[449,450]$. The important in vivo findings are summarized per strain in Table 1.

In this study, we addressed the effect of immune environment and age on the in vitro response by splenocytes and macrophages to bacterial supplementations.

Table 1. Properties of tested bacterial strains.

\begin{tabular}{|c|c|c|c|c|}
\hline Species & Strain & Origin & In vivo activities & Ref. \\
\hline $\begin{array}{l}\text { Lactobacillus } \\
\text { plantarum }\end{array}$ & $\begin{array}{l}\text { WCFS1 } \\
\text { DSMZ20174 } \\
\text { ATCC BAA-793 }\end{array}$ & $\begin{array}{l}\text { Human } \\
\text { pharynx }\end{array}$ & $\begin{array}{l}\text { Anti-allergic (Betv1) } \\
\text { Pro-allergic (peanut) } \\
\text { Increased \# splenic Tregs } \\
\text { Reduced HFD-induced pathology } \\
\text { Trend to protection in TNBS colitis }\end{array}$ & [451-458] \\
\hline $\begin{array}{l}\text { Lactobacillus } \\
\text { casei }^{T}\end{array}$ & $\begin{array}{l}\text { BL23 } \\
\text { plasmid-cured } \\
\text { ATCC393 }\end{array}$ & $\begin{array}{l}\text { Dairy } \\
\text { products }\end{array}$ & Protective in TNBS colitis & {$[443,444]$} \\
\hline $\begin{array}{l}\text { Lactococcus } \\
\text { lactis }\end{array}$ & MG1363 & $\begin{array}{l}\text { Dairy } \\
\text { products }\end{array}$ & Not reported & $\begin{array}{l}{[444,459,} \\
460]\end{array}$ \\
\hline $\begin{array}{l}\text { Bifidobacterium } \\
\text { breve }^{T}\end{array}$ & $\begin{array}{l}\text { ATCC15700 } \\
\text { DSM20213 }\end{array}$ & $\begin{array}{l}\text { Infant } \\
\text { intestine }\end{array}$ & ${ }_{1}^{\text {No protection against } P \text {. aeruginosa }}$ & {$[461]$} \\
\hline $\begin{array}{l}\text { Bifidobacterium } \\
\text { infantis }^{T}\end{array}$ & $\begin{array}{l}\text { ATCC15697 } \\
\text { DSM20088 }\end{array}$ & $\begin{array}{l}\text { Infant } \\
\text { intestine }\end{array}$ & 2 & \\
\hline $\begin{array}{l}\text { Akkermansia } \\
\text { muciniphila }^{T}\end{array}$ & ATCC BAA-835 & $\begin{array}{l}\text { Adult } \\
\text { intestine }\end{array}$ & $\begin{array}{l}\text { Protection against obesity and T2D } \\
\text { Increased \# goblet cells and } \\
\text { VAT Tregs }\end{array}$ & $\begin{array}{l}{[452,462-} \\
464]\end{array}$ \\
\hline
\end{tabular}

\footnotetext{
T type strain defines a species and is representative of that species. Literature findings referring to the same species but with other strain numbers is indicated with a number: ${ }^{1}$ anti-allergic [446]; ${ }^{2}$ protection against Rotavirus and protection against DSS colitis [465, 466]. DSS = dextran sulfate sodium; GF = germfree; HFD = high fat diet; T2D = type 2 diabetes; TNBS = 2,4,6-trinitrobenze sulfonic acid; Treg = regulatory $\mathrm{T}$ helper cell; VAT = visceral adipose tissue.
} 


\section{MATERIALS AND METHODS}

\section{Bacterial cultures}

L. plantarum, L. casei, L. lactis, B. breve, B. infantis and A. muciniphila were grown until stationary phase was reached. L. lactis was grown on M17 medium (Merck, Darmstadt, Germany), A. muciniphila was grown on mucin-based medium [467], whereas the other four strains were grown on MRS medium (Merck). B. breve, B. infantis, and $A$. muciniphila were cultured under strictly anaerobic conditions. Viability and colony forming units (CFU) were checked by plating and measuring the $\mathrm{OD}_{600}$. The bacterial batches were freshly cultured for each individual experiment.

\section{Mice}

Male 7-week-old C57BI/6J mice were purchased from Harlan (The Netherlands). Male 18-month-old C57BI/6J mice were purchased from Janvier (France) and housed for 7 months at the animal facility of Wageningen University. All animals were specific pathogen free, and had free access to water and feed. Mice were fed D12450B diet (Research Diet Services, Wijk bij Duurstede, The Netherlands). All experiments were performed with approval of the animal care and use committee of Wageningen University. Young mice were sacrificed between 8-12 weeks of age and aged mice at 25 months of age.

\section{Spleen cultures}

Mice were sacrificed by anesthesia with isoflurane. Spleen single cells suspensions were obtained by disrupting the organs and passing cells through a cell strainer. Lysis of erythrocytes was performed using RBC lysis buffer (eBioscience, San Diego, CA, USA). About $10^{6}$ fresh total spleen cells were cultured in 48-wells plates and stimulated with $200 \mathrm{ng} / \mathrm{mL}$ LPS (E. coli 055:B5, Sigma-Aldrich, Zwijndrecht, The Netherlands) $+50 \mathrm{ng} / \mathrm{mL}$ recombinant mouse IFN- $\gamma$ (BioLegend, San Diego, CA, USA) or $0.1,1$ or $10 \mathrm{CFU}$ of viable bacterial strain cultures per splenocyte in RPMI 1640 medium (Gibco, Breda, The Netherlands) supplemented with $10 \%$ FCS (Gibco), $100 \mathrm{U} / \mathrm{mL}$ penicillin-streptomycin (Gibco), and $50 \mu \mathrm{M} \beta$-mercaptoethanol (Sigma-Aldrich). IFN- $\gamma$ was included to mimic an immune-activated state and to enhance the response by e.g. macrophages and $T$ cells present in the culture. Supernatants were harvested after 24-hour stimulation and stored maximally one month at $-20^{\circ} \mathrm{C}$ for cytokine analysis. 
Chapter 5

\section{Macrophage cultures}

Femora were flushed to obtain BM cells, which were passed through a cell strainer. Half a million BM cells were cultured in 24-well plates in the presence of $10 \%$ Ladmacconditioned medium [468] and $10 \mathrm{ng} / \mathrm{mL}$ recombinant mouse M-CSF (CSF-1, eBioscience) to generate bone marrow-derived macrophages (BMDM, hereafter called M0 macrophages). After 6 days, BMDM were stimulated overnight with $200 \mathrm{ng} / \mathrm{mL}$ LPS as a positive control or with viable bacterial strains (1 CFU per $1 \mathrm{BMDM}$ ). To generate BMDM-IFN (hereafter called M-IFN macrophages), $50 \mathrm{ng} / \mathrm{mL}$ IFN- $\mathrm{y}$ was added to the standard culture medium along with overnight (18-hour) stimulation. Cells were harvested for flow cytometry and supernatants were stored frozen for later cytokine analysis.

\section{Flow cytometry}

Flow cytometry was performed using standard procedures. Macrophages were stained with monoclonal antibodies for MHC-II-FITC (M5/114.152, eBioscience), F4/80-PerCP-Cy5.5 (BM8, eBioscience), CD11C-PE-Cy7 (N418, eBioscience), CD86APC (GL1, eBioscience), CD11b-APC-Cy7 (M1/70, BD Biosciences, Erembodegem, Belgium), and CD54-PB (ICAM-1; YN1/1.7.4, BioLegend). Fluorescent signals were acquired using a BD FACSCanto II (BD Biosciences). Data were analyzed with FlowJo vX.07 (Tree Star) software.

\section{Cytokine measurements}

IL-12p70, TNF, IFN- $\gamma$, CCL2/MCP-1, IL-10, and IL-6 concentrations in the supernatants of splenocyte and BMDM cultures were determined using the Cytometric Bead Array (CBA) Mouse Inflammation Kit (BD Biosciences), according to the manufacturer's instructions.

\section{Statistical analysis}

Data are expressed as average \pm SEM, unless otherwise stated. One-way ANOVA and subsequent Bonferroni post hoc tests were performed to test differences between bacterial strains. GraphPad Prism version 5.0.3 (San Diego, CA, USA) was used to perform statistical tests. Significant differences were indicated by asterisks: ${ }^{*}=p<0.05$; ${ }^{* *}=p<0.01 ;{ }^{* * *}=p<0.001$. 


\section{RESULTS}

\section{Dose-response relation for bacterial stimulation on splenocytes}

To determine the dose-response effects of bacterial strains on the immune cells studied, we used cultures of splenocytes, and after 24-hour stimulation measured production of cytokines produced under inflammatory conditions. Splenocytes were incubated with three concentrations of bacteria (ratio bacteria:splenocytes of 1:10, 1:1, 10:1). A positive dose-response relation was observed for all bacterial strains (Figure 1). Both Bifidobacterium strains and $A$. muciniphila induced the highest levels of IL-10 (to $200 \mathrm{pg} / \mathrm{mL}$ ), TNF (to $1000 \mathrm{pg} / \mathrm{mL}$ ), IL-6 (to $100 \mathrm{pg} / \mathrm{mL}$ ), as well as CCL2/MCP-1 (to $100 \mathrm{pg} / \mathrm{mL}$ ). After applying the highest dose of some of the bacterial strains, or after applying positive control LPS+IFN- $y$, only low levels of IL-12p70 were detected. These low levels of IL-12p70 were expected, as IFN- $y$ is necessary to induce IL-12p70 (instead of IL-12p40) production [469, 470].

We calculated the IL-10/TNF ratio for each bacterial dose as a measure of anti- and pro-inflammatory cytokine balance (Figure 1). The Bifidobacterium strains and $A$. $m u$ ciniphila showed an increasing IL-10/TNF ratio with increasing dose, implicating that higher doses of those strains preferentially stimulated IL-10 production rather than TNF production. Both Lactobacillus strains and L. lactis, in contrast, showed a decreasing IL-10/TNF ratio with increasing bacterial dose.

These data indicate that two distinct subgroups of bacterial strains are identified, independent of the applied dose: Lactobacillus strains and L. lactis, inducing lower levels of inflammatory cytokines than the Bifidobacterium strains and $A$. muciniphila. These different profiles are also reflected in decreasing and increasing IL-10/TNF ratios, with increasing bacterial dose.

\section{Differential phenotypic response by macrophages on different bacterial strains}

Next we studied the effects of the distinct types of bacterial strains on naïve (M0) and IFN-Y-stimulated (M-IFN) BMDM in order to determine whether the immune environment of the macrophages is of influence on the responsiveness. As sentinel cells, macrophages are known to be fast and potent in their responses (as confirmed in our experiments). To detect differences between bacterial strains at the most sensitive level, we incubated the macrophages overnight with 1 CFU per macrophage, and assessed phenotypic changes by flow cytometry. Mature macrophages were defined as

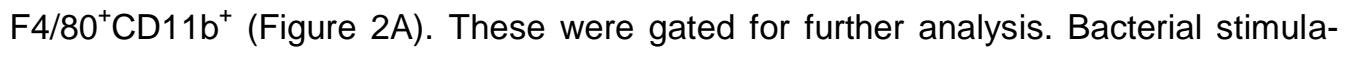
tion and IFN-y stimulation did not affect CD11b expression of macrophages from young mice, allowing appropriate gating after all stimulations (Supplementary 

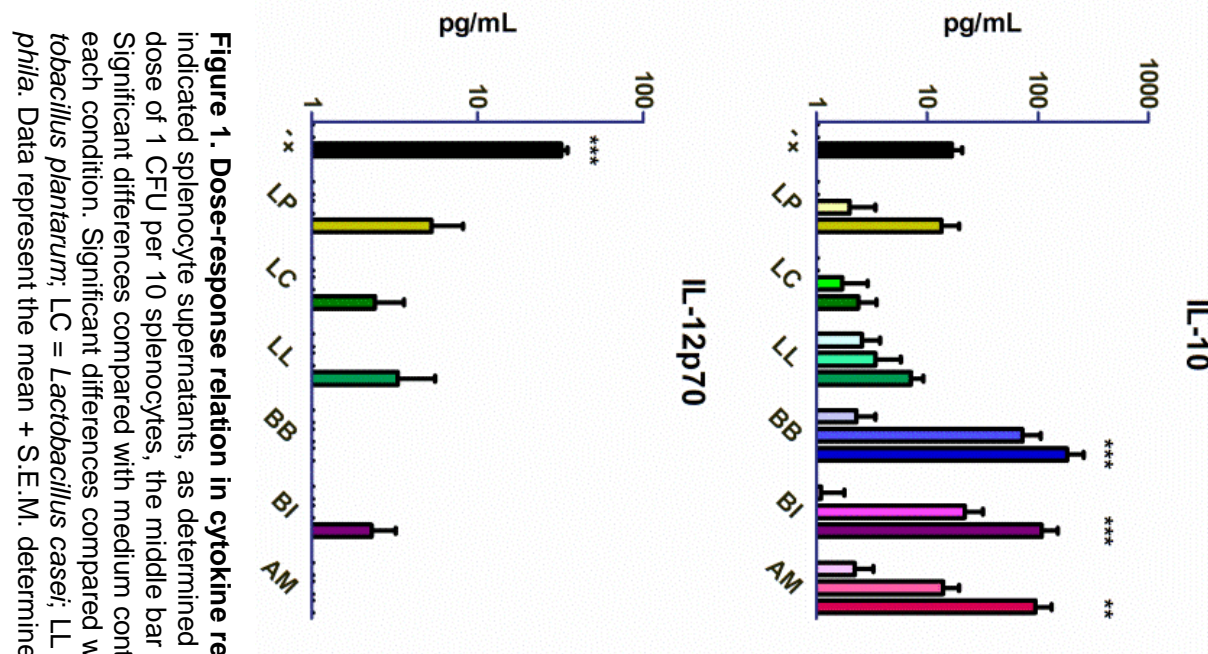

₹
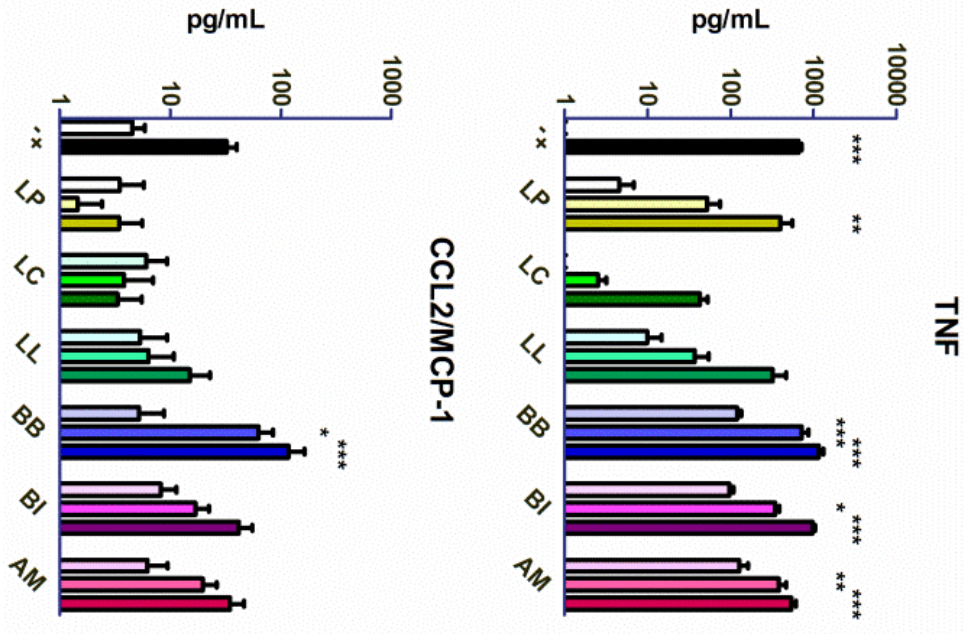

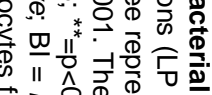

言舟

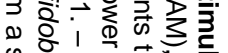

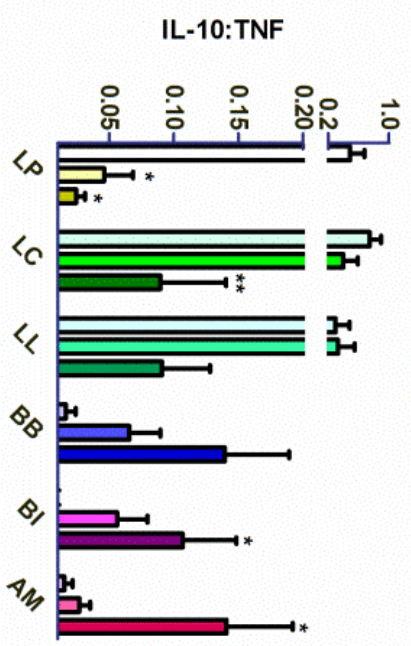

$\mathrm{pg} / \mathrm{mL}$

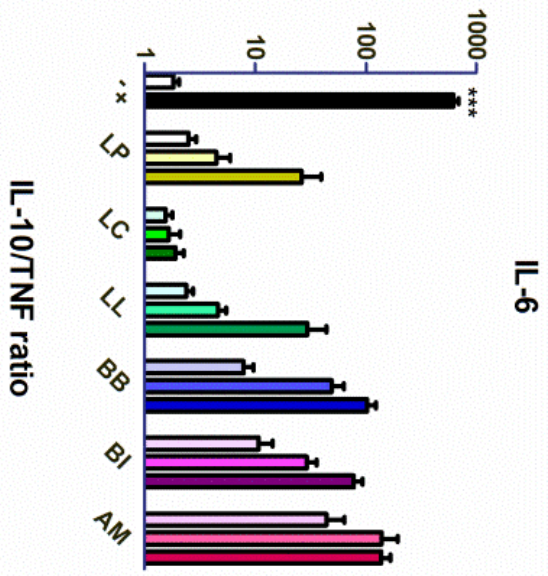




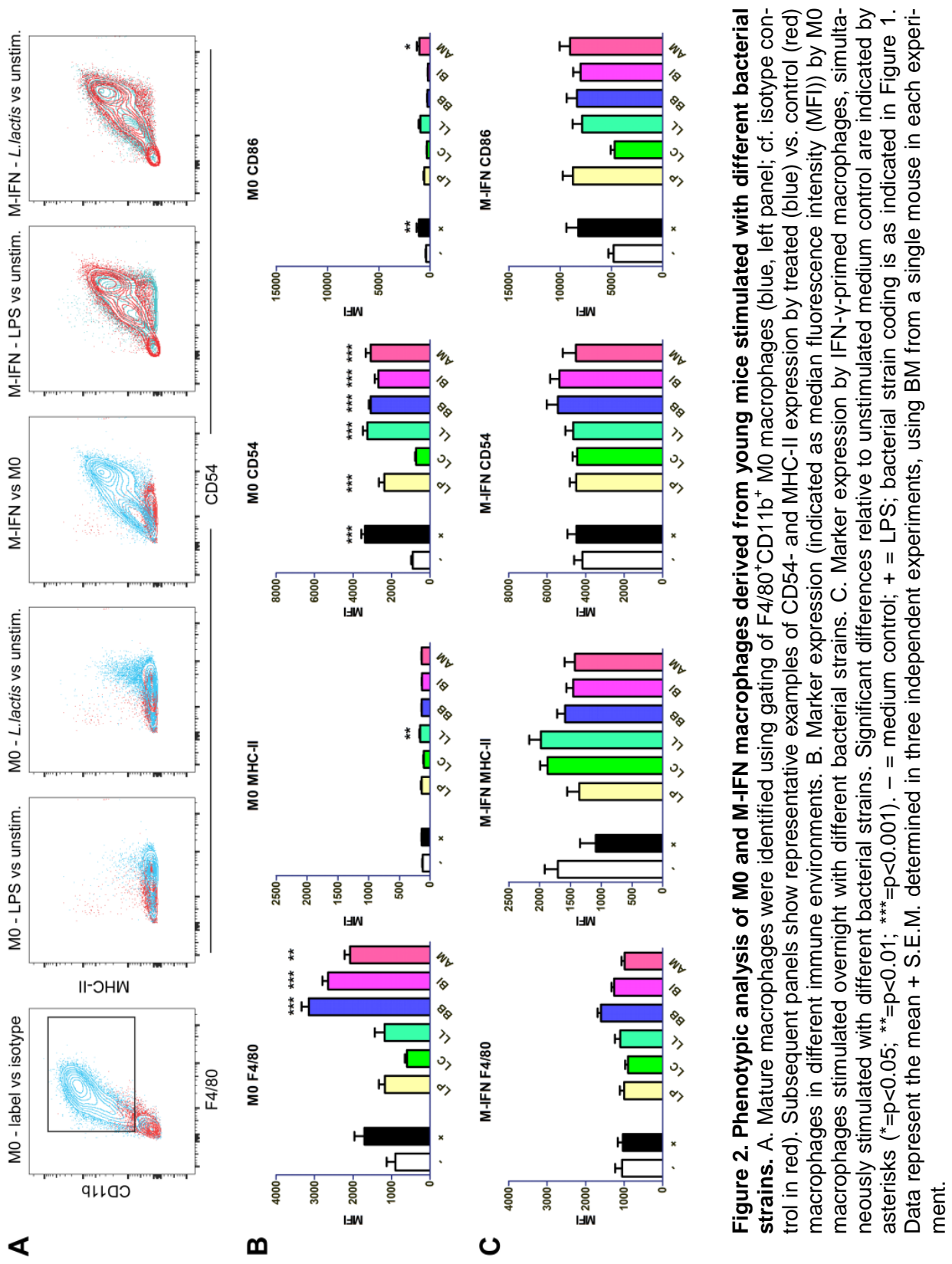


Figure 1). M0 macrophages responded phenotypically upon incubation with LPS or bacteria by increasing CD54 expression, and frequently up-regulating F4/80. In incidental cases MHC class II or CD86 expression was found to be increased in M0 macrophages (Figure 2B). Most marked changes were observed with $A$. muciniphila (CD54, CD86, and F4/80), L. lactis (CD54 and MHC-II; Figure 2B) and both Bifidobacterium strains (CD54 and F4/80). L. plantarum induced higher expression of only CD54, as compared with the control. L. casei stimulation caused no phenotypic changes in $\mathrm{M} 0$ macrophages. Priming of macrophages with IFN- $y$ caused a prominent increase in CD54, CD86, and MHC-II expression (Figure 2C). In general, simultaneous stimulation with bacteria and IFN- $y$ did not further change surface marker expression on M-IFN of CD11b, CD54, F4/80, and MHC-II (Figure 2C). One-way ANOVA for analysis of variance revealed differences in CD86 expression, comparing all conditions $(p=0.002)$. A trend for increasing CD86 expression was observed for all bacterial stimulations except $L$. casei.

Together, these data demonstrate that M0 macrophages respond phenotypically to bacterial stimulation. IFN-y stimulation exerts a prominent effect on macrophage phenotype, which is hardly influenced by simultaneous bacterial stimulation. Furthermore,

a clear qualitative difference between bacterial strains is observed, with $L$. casei being the only bacterial strain not changing macrophage phenotype at all. Finally, based on changes in $\mathrm{F} 4 / 80$ expression induced in $\mathrm{M} 0$ macrophages, the same two subgroups of bacteria (L. plantarum, L. casei, and L. lactis versus B. breve, B. infantis, and A. muciniphila) were observed as identified before.

\section{Bacterial exposure differentially stimulates macrophage cytokine secretion}

Upon activation, macrophages are capable of producing high amounts of cytokines. Therefore, we studied cytokine secretion by differentially stimulated macrophages. IL10 secretion was only detectable when M0 macrophages were stimulated with LPS, the bifidobacteria or A. muciniphila (Figure 3). This secretion profile was similar in MIFN macrophages with IL-12p70 instead of IL-10 secretion. The highest IL-10 production in M0 was observed upon stimulation with $A$. muciniphila. TNF and IL-6 production was observed in both $\mathrm{M} 0$ and M-IFN macrophages, in particular after incubation with the bifidobacteria and $A$. muciniphila. CCL2/MCP-1 production was increased upon $B$. infantis and $A$. muciniphila incubation, in $\mathrm{MO}$ macrophages as well as in $\mathrm{M}$ IFN macrophages. The IL-10/TNF ratio of M0 macrophages revealed that $A$. muciniphila mostly increased IL-10 production (compared to TNF production; Figure 4). Next to the IL-10/TNF ratio, we calculated a production index, by normalizing IL-10+TNF production upon bacterial stimulation against IL-10+TNF production upon LPS stimulation. The production index indicates a clear difference in induction of IL-10 and TNF production between the tested lactobacilli and Lactococcus on one side, and the 
bifidobacteria and Akkermansia on the other side. Application of IL-10/TNF ratio for MIFN is impossible, because IFN- $\gamma$ priming blocks IL-10 secretion nearly completely in most conditions.

These data show differential effects of bacterial strains on cytokine production by macrophages. As in splenocyte cytokine production and macrophage phenotype, a division between the Lactobacillus strains and Lactococcus on one hand, and the Bifidobacterium strains and Akkermansia on the other hand is indicated by the greater capacity of the latter to induce inflammatory cytokines, in particular TNF and IL-6.

\section{Age-dependent shift in cytokine production by splenocytes}

To investigate whether the response to bacterial strains does change during aging, we isolated spleens from aged (25-months-old) mice, and compared the reactivity of splenocytes to that of young (8-12-weeks-old) mice. Splenocytes were incubated with bacteria in a 1:1 ratio. Aged splenocytes produced markedly higher levels of IL-10, as compared with young splenocytes, upon stimulation with virtually any of the bacterial strains (Figure 5, Table S1). Upon LPS+ IFN-y stimulation, used as positive control for inflammatory cytokine induction, production of TNF, IL-6, IL-12p70, and CCL2/MCP-1 was lower in aged splenocytes compared with young splenocytes. This downmodulation was not generally observed upon stimulation with bacteria. In particular, TNF production was enhanced in aged splenocytes in response to bacteria (Table S1). The response to $L$. lactis, the Bifidobacterium strains and $A$. muciniphila slightly changed with age. $B$. breve-stimulated splenocytes from aged mice in particular did not show changes in cytokine responses compared to young splenocytes; only CCL2/MCP-1 production was decreased. The IL-10/TNF ratio was greater in aged splenocytes than in young splenocytes for most of the conditions, except for $L$. casei (Figure 5). L. casei showed an increased cytokine induction in aged splenocytes, as compared with young splenocytes. The levels induced by L. casei, however, did not reach those induced by the Bifidobacterium strains or Akkermansia (Table S1).

These data show an age-dependent shift of cytokine responses towards IL-10. The increase is stronger when comparing IL-10 to TNF, IL-6, and CCL2/MCP-1 production. It also indicates that certain bacterial strains exert a different effect on aged immune cells than on young immune cells. Particularly, L. casei showed increased induction of responses in aged splenocytes, while it was non-responsive in young immune cells. 
Chapter 5

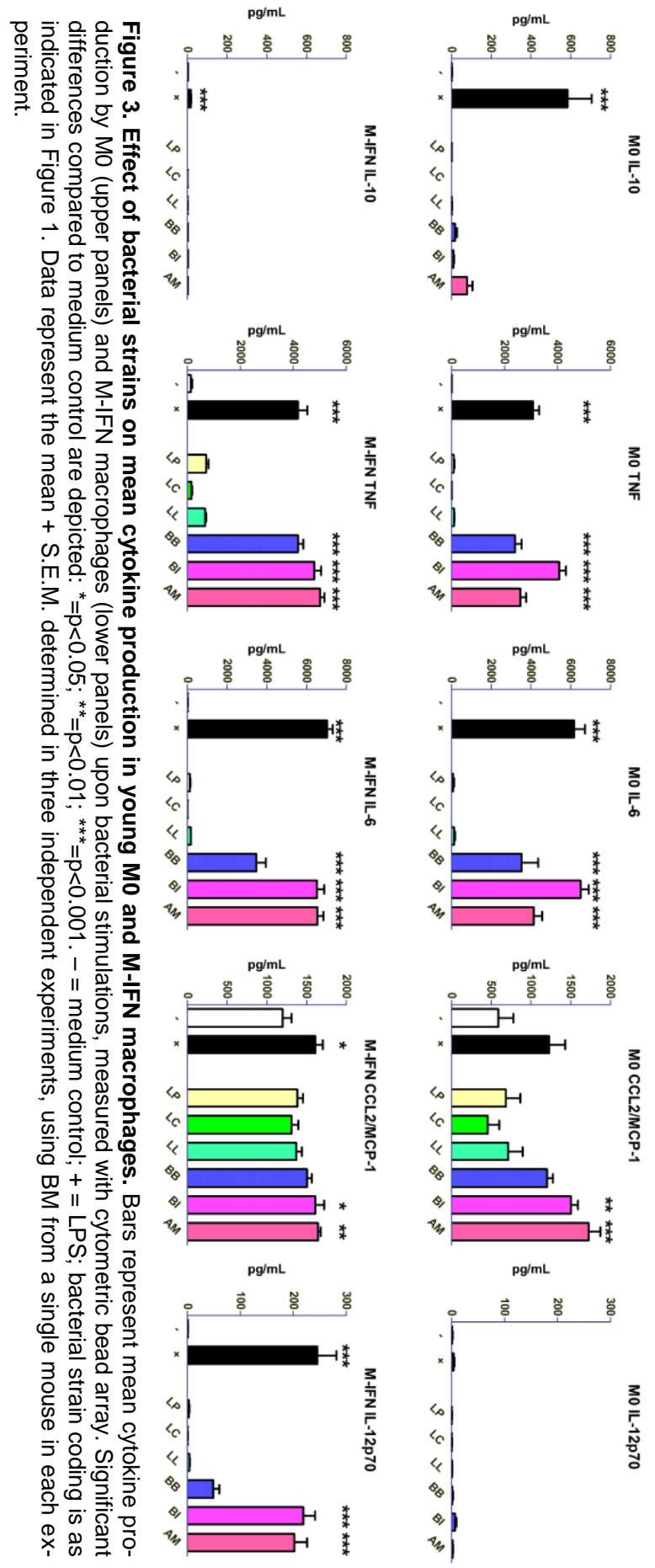


IL-10/TNF ratio M0

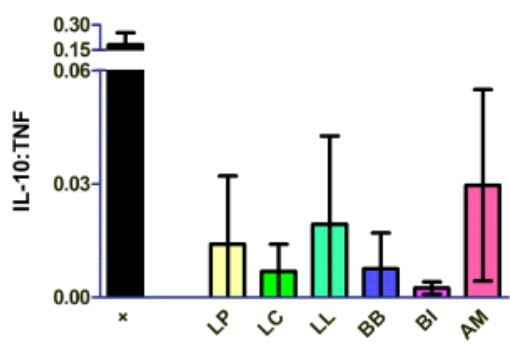

Production index MO

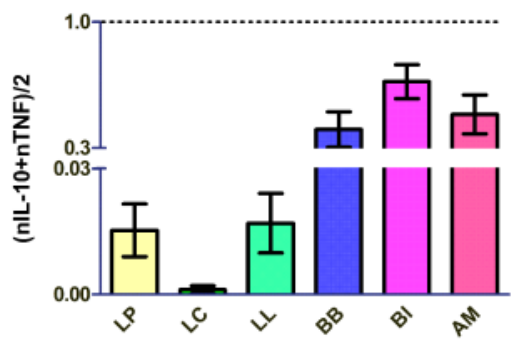

Figure 4. IL-10/TNF ratio and cytokine production index of young M0 macrophages stimulated with different bacterial strains. The production index indicates normalized IL-10 + normalized TNF levels, divided by 2. Normalization was performed against LPS (resulting in a value of 1 for LPS). + = LPS; bacterial strain coding is as indicated in Figure 1. Error bars represent $95 \%$ confidence intervals.

IL-10

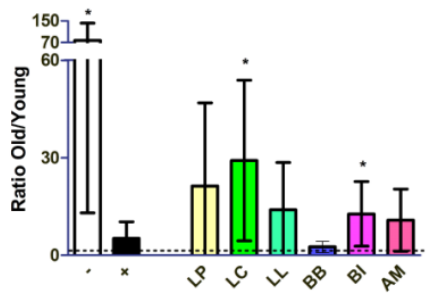

CCL2/MCP-1

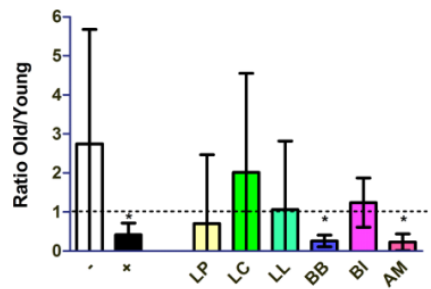

TNF

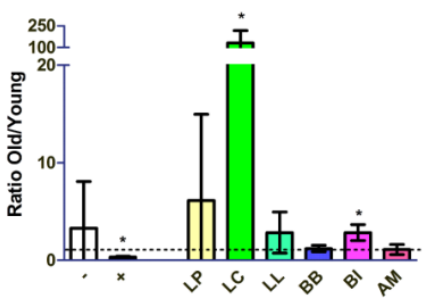

IL-10/TNF ratio

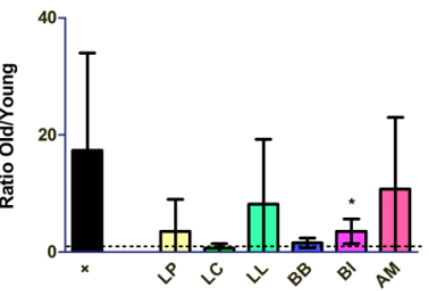

IL-6

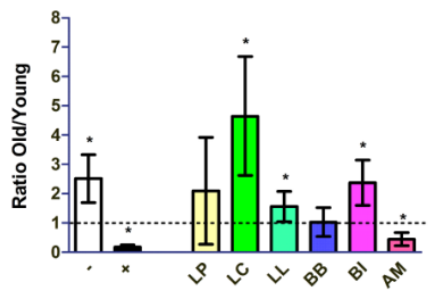

Figure 5. Aging affects cytokine responses of splenocytes, in particular by increasing IL-10 levels. Cytokine levels measured in cultures of aged splenocytes stimulated with different bacterial strains are expressed as a ratio to cytokine levels from similarly stimulated young splenocytes. $-=$ medium control; $+=$ LPS+IFN- $\gamma$; bacterial strain coding is as indicated in Figure 1. Data represent the means of six independent experiments. Error bars represent $95 \%$ confidence intervals.

\section{Aging affects macrophage phenotype and cytokine responses to bacteria}

Next, we compared the effects of bacterial strains on macrophages from aged mice to those of young in surface marker expression and cytokine production. Due to altered signal acquisition in flow cytometry (caused by technical maintenance in between experiments), we were unable to compare values directly side-by-side, but approached this by normalizing MFI values for each surface marker against the stable surface marker CD11b. F4/80 and MHC-II were upregulated with age under all conditions (Figure 6). The division between the Lactobacillus strains and L. lactis stimulation on the one hand, and the Bifidobacterium strains and A. muciniphila stimulation on the other hand, was also observed in various aspects in aged macrophage phenotypic 
Chapter 5

changes. Compared to unstimulated control, stimulation with L. lactis, both Bifidobacterium strains, or $A$. muciniphila resulted in a relatively lower increase of F4/80 expression in aged M0 macrophages than in young M0 macrophages, and a lower increase of MHC-II, CD54, and CD86 expression in aged M-IFN macrophages than in young M-IFN macrophages (Figure 6). In contrast, stimulation with either of the Lactobacillus strains resulted in a relatively higher increase of F4/80 expression in aged M0 macrophages than in young M0 macrophages.

Changes for different stimuli were also observed in cytokine profiles when macrophages from young and aged mice were compared. IL-10 secretion by M0 macrophages increased with age in response to most bacterial stimulations (Figure 7, Table S2). In response to LPS, however, IL-10, TNF, and IL-6 levels were lower in aged BMDM, whereas CCL2/MCP-1 levels were higher, as compared with young BMDM. Relative production of TNF was reduced in aged M0 macrophages upon exposure to the Bifidobacterium strains and A. muciniphila incubation, but not in L. plantarum and L. casei-stimulated M0 macrophages (Figure 7). L. lactis-induced cytokine responses by aged macrophages were without exception down or similar compared to young macrophages. Similarly, IL-6 production was reduced with aging in M0 and M-IFN macrophages upon some stimulations (except for $L$. casei-stimulated macrophages and $L$. plantarum-stimulated M-IFN macrophages; Figure 7 ). It is noteworthy that $L$. casei induced an increased cytokine response in macrophages derived from old mice compared to those from young mice. However, in absolute values, the differences are rather small (Table S2, S3).

In general, the differential effects of the previously identified subgroups of bacteria are also evident in macrophages from aged mice in the relative increase (Lactobacillus strains) or decrease (Bifidobacterium strains and Akkermansia) of surface markers and cytokines. However, the difference between the various bacterial strains observed in young cultures (Figure 1-4), is less pronounced in aged cultures (Table S2, S3).

These data indicate that aging impacts the response of splenocytes and macrophages to bacteria. 
Effect of age on interaction between immune cells and bacterial strains in vitro
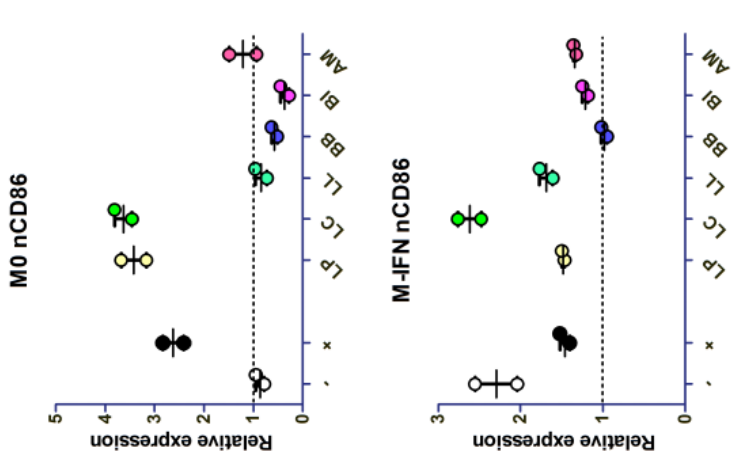

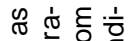

이응 은

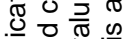

중

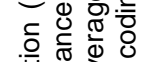

证

Nㅗㅇ 을

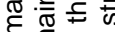

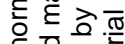

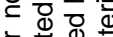

Ф) 은 은

更

월 웜
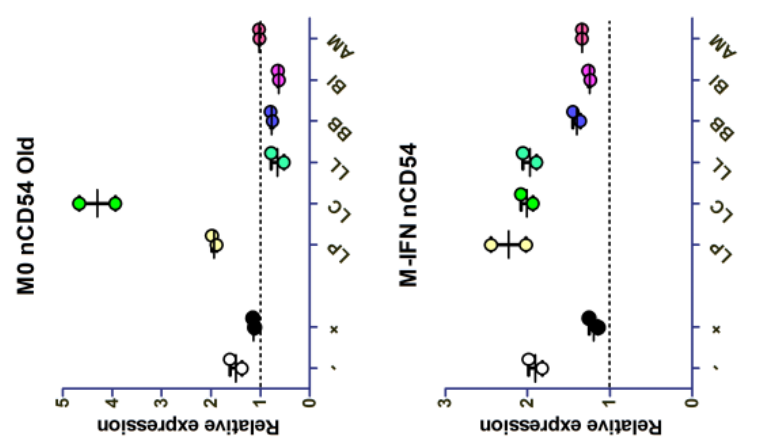

कृ

d $\mathrm{O}+$

원 흥 등

․ㅡ은

สั

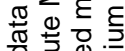

잉

응

충

는

\& \& $\&$ व

등 그을

\$히

車
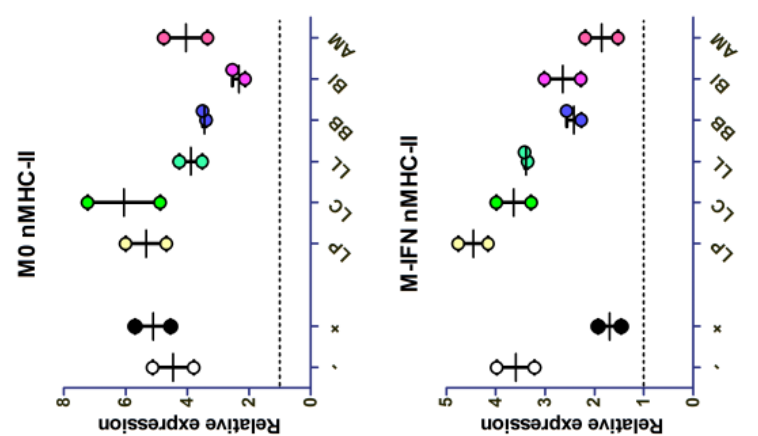

응유 중

운 을 흫

저읃

है

z들

훈웜은

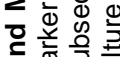

ธั

을 ⿺⿻一𠃋火

ডั

क क

옹응 잉

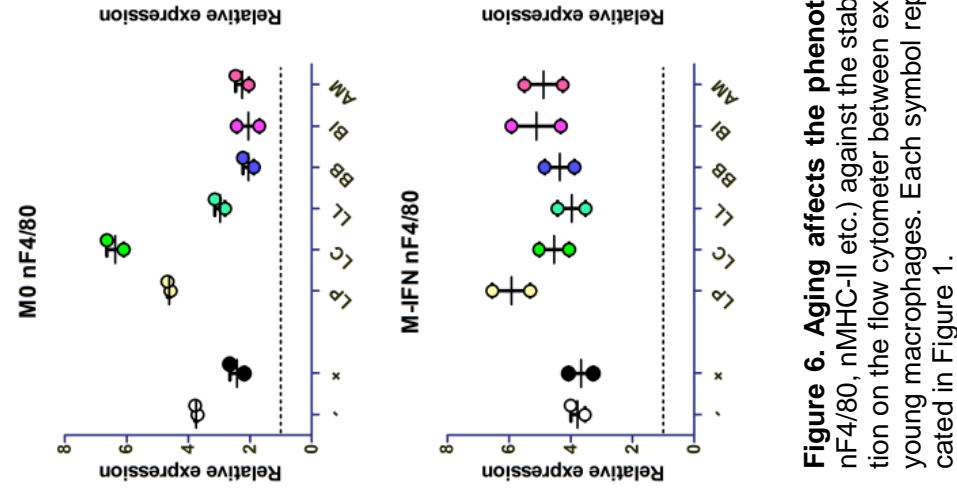




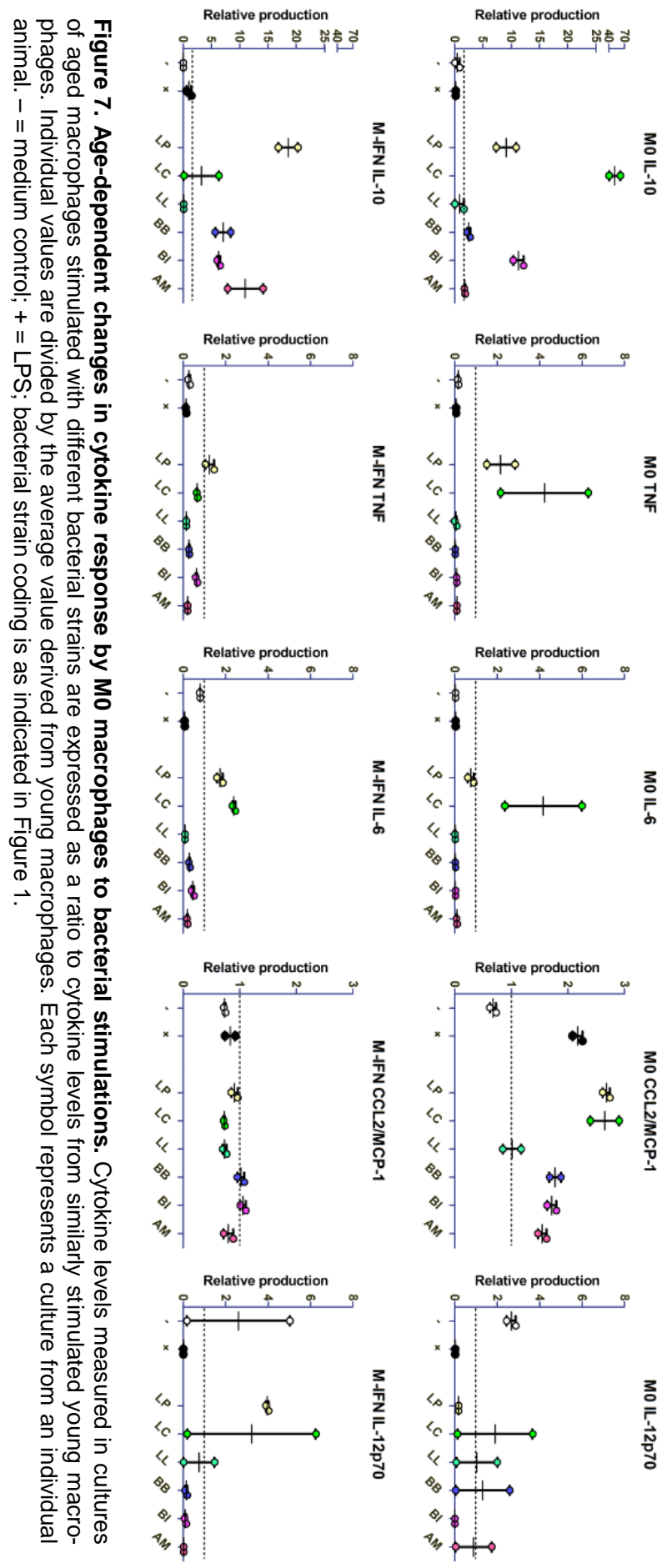




\section{DISCUSSION}

In this study, we used splenocyte and BM-derived macrophage cultures to investigate the effect of immune environment and age on immune activation induced by bacteria potentially used as food supplement. Differential reactivity to two groups of bacterial strains was consistently observed in splenocyte and macrophage cultures, i.e. the Lactobacillus plantarum WCFS1 and Lactobacillus casei BL23 strains and Lactococcus lactis MG1363 inducing rather moderate responses compared to the positive control (LPS \pm IFN- $\gamma$ ), the Bifidobacterium breve ATCC15700 and Bifidobacterium infantis ATCC15697 strains, and Akkermansia muciniphila ATCC BAA-835. Aging alters the inflammatory response of immune cells to bacteria. The IL-10 secretion, in particular, by aged splenocytes was greater than the IL-10 secretion by young splenocytes. The same trend for greater IL-10 production was observed in BMDM, in particular when comparing IL-10 production to IL-6 and TNF production.

In probiotic research PBMCs and dendritic cells are commonly used to select immuneactive bacterial strains [444, 471, 472]. These studies did not take into account different immune environment and age of responder cells. The rationale in the current study was to compare the responsiveness of $\mathrm{MO}$ and $\mathrm{M}$-IFN, i.e. different immune environments. M0 macrophages, i.e. naïve or M-CSF-primed macrophages reacted differently to the same bacterial strains as M-IFN macrophages. Our data therefore suggest to focus not only on one type of immune environment but on different types of mononuclear phagocytic cells to gain insight into the immune-potentiating effects of bacterial strains.

Many previous studies testing direct bacteria-immune interactions in vitro made use of human PBMC in which IL-10/IL-12 ratios were taken as a measure for pro- or antiinflammatory capacity [457, 472]. In the current study, we used splenocytes as a PBMC collection. We could not apply IL-10/IL-12 ratios as IL-12p70 is produced in very low amounts in mice, and only induced in the presence of IFN-y plus a simultaneous stimulation such as LPS. We considered measuring IL-12p40 values instead, but deemed such results difficult to interpret in inflammatory terms, since p40 subunits may occur either as single chains, as heterodimers with p35, p19 or other partners with pro- or unknown inflammatory activity, or even as inhibitory p80 dimers [473, 474]. Therefore, we applied IL-10/TNF ratios. From a previous study, it is clear that TNF and IL-12p70 production by human monocyte-derived DC is closely correlated [471], and also in our study we have an indication for such a correlation (Figure 3).

Despite the conceptual differences, comparing responses by freshly isolated spleen cells with those by BMDM, the results obtained in these culture systems generally were in close agreement. The bacterial strains that induced the greatest responses upon direct contact, responded similarly in splenocyte and in macrophage cultures. However, calculated per cell, splenocyte cultures produced much less cytokines than 
Chapter 5

macrophage cultures. Therefore, higher doses might be needed to measure certain cytokines in splenocyte supernatants. Most cytokine levels were below or around detection limits when using the lowest dose of certain bacterial strains, but most of the cytokines could be detected when using a higher dose. This dose-response relation is important when applying bacteria in vivo. A limitation of this study is that we only determined the response of macrophages to a single bacterial dose, and thus could not establish whether the two groups of bacteria showed a similar decreasing and increasing IL-10/TNF ratio, respectively, upon stimulation with increasing bacterial doses. However, it is evident that BMDM are more potent in their response to bacteria compared to splenocytes. Therefore, the optimal dose for stimulating splenocyte cultures is probably much higher than for macrophages. In addition, measuring multiple time points after addition of bacterial stimulations would have given insight in the kinetics of the observed immune responses.

Based on macrophage phenotype, and splenocyte and macrophage cytokine responses, we identified two subgroups of bacteria (L. plantarum WCFS1 and L. casei BL23 versus $B$. breve ATCC15700, B. infantis ATCC15697 and A. muciniphila ATCC BAA-835). It is remarkable, that $A$. muciniphila, as the only tested gram-negative species, induces similar responses as the Bifidobacterium strains. Furthermore, the first group represents facultative anaerobic bacteria, whereas the second group includes strictly anaerobic bacteria. L. lactis MG1363 resides mostly in the first group, but sometimes induced different responses, depending on the culture system we used. It is the only included strain which is generally recognized as safe for human consumption, but non-probiotic [459]. We demonstrate that this strain is inducing a weak inflammatory response, which could support its widely studied function in bioactive molecule delivery in the gut [445]. With regard to the differences between bacterial strains, the choice for standardizing the doses based on CFU might have influenced the response triggered by the different strains. For instance, the biomass per CFU will most likely differ between bacterial strains, which might have had an impact on the triggered response.

IL-10/IL-12 or IL-10/TNF ratios can be valuable to assess pro- or anti-inflammatory capacities of bacterial strains in order to predict in vivo responses [444]. They are, however, inappropriate when applying to e.g. aging individuals, considering the fact that Toll-like receptor (TLR)-mediated responses are affected by aging [475]. We also observed that the IL-10/TNF ratio in response to bacterial stimulations changes with age, which is probably mediated by age-acquired TLR signaling defects $[476,477]$ and dysregulated expression of the negative feedback regulator of TLR signaling miR146a [478]. Besides, responsiveness to LPS is reduced, supporting the evidence for TLR4-signalling defects in aged immune cells [479]. In general, IL-10 production was markedly increased in splenocytes and macrophages. This was reported by several 
previous studies $[475,476]$. Further research is necessary to investigate the underlying mechanisms. On the bacterial side, mutants could play an important role by elucidating the role of surface molecules or secreted factors (e.g. short-chain fatty acids) by bacteria [480]. On the host side, IL-10 blocking or IL-12p70 supplementation might be interesting treatments to restore the response to bacterial supplementation in aged cells. A technical note of caution related to the interpretation of age-related differences is that the supplier of aged mice was different from the supplier of young mice, which most likely resulted in a difference in the microbiota composition. The microbiota composition has a crucial role in priming several immune cell types [126]. The fact that the obtained cells from young and aged mice could have been differently primed in vivo, might have influenced the outcome of this study.

Aging effects found in splenocytes (isolated as primary cells from aged mice) are similar to those observed in BM-derived macrophages. The latter are derived from aged BM precursor cells, which have undergone multiple cell cycles in vitro, up to 8-9, before being exposed to bacteria. The finding that similar age-related changes have been retained in these cells compared to freshly isolated splenocytes implies that differences, probably due to epigenetic changes during aging [481-483], are preserved during in vitro multiplication and differentiation of the cells. In accordance, it has been reported that hematopoietic stem cells are epigenetically dysregulated with age [65, 484]. Another study, using a spermatogonial stem cell culture of over two years, demonstrated a remarkable imprinting potential and a resilience to epigenetic modifications in vitro [485].

Taking the differential effects of age on the immune response into account, it can be envisaged that probiotics that are beneficial in children, might have no favorable effect in elderly, and vice versa. For example, the reported beneficial effect of probiotic cheese containing L. rhamnosus HN001 and L. acidophilus NCFM in elderly [170], should be tested in children and adults to confirm its positive effect in different age classes. Acknowledging the generic adverse effects of age on functions of all body cells, including immune cells, we expect different effects of probiotics in children, adults, and elderly. A side-by-side study with children, adults, and elderly would thus provide insight into the in vivo interaction between probiotics and host, and the effect of age on this interaction.

In conclusion, we observed that the response of splenocytes and macrophages on stimulation with different bacterial strains is altered with aging. It underlines the caution which is needed when translating findings in young immune cells or individuals to aged cells or elderly individuals. 
Chapter 5

\section{ACKNOWLEDGEMENTS}

Ben Meijer and Steven Aalvink are acknowledged for support during experimental work.

\section{SUPPLEMENTAL INFORMATION}
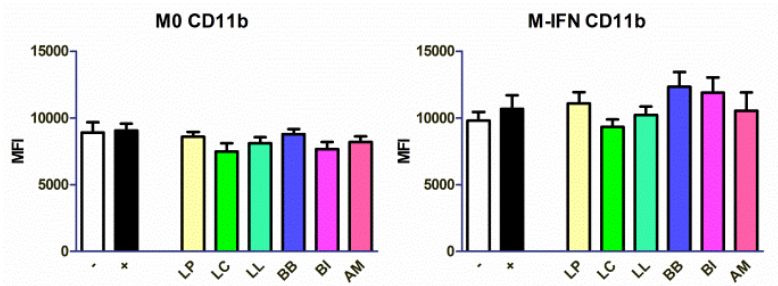

Figure S1. CD11b expression unaltered after stimulation of M0 and M-IFN macrophages derived from young mice with bacterial strains stimulated with different bacterial strains. CD11b expression is indicated as median fluorescence intensity (MFI). $-=$ medium control; + = LPS; bacterial strain coding is as indicated in Figure 1. Data represent the mean + S.E.M. determined in three independent experiments. 


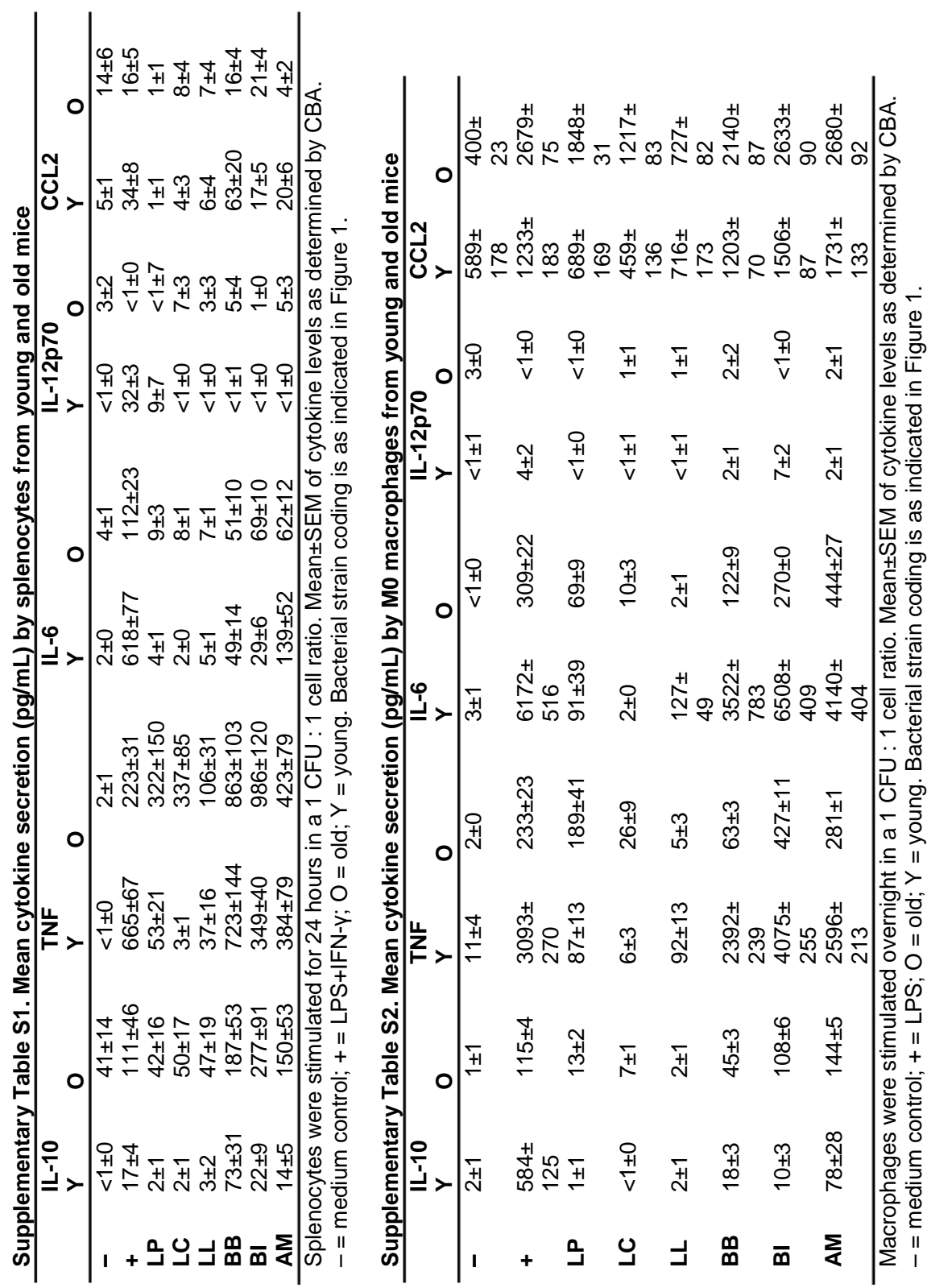




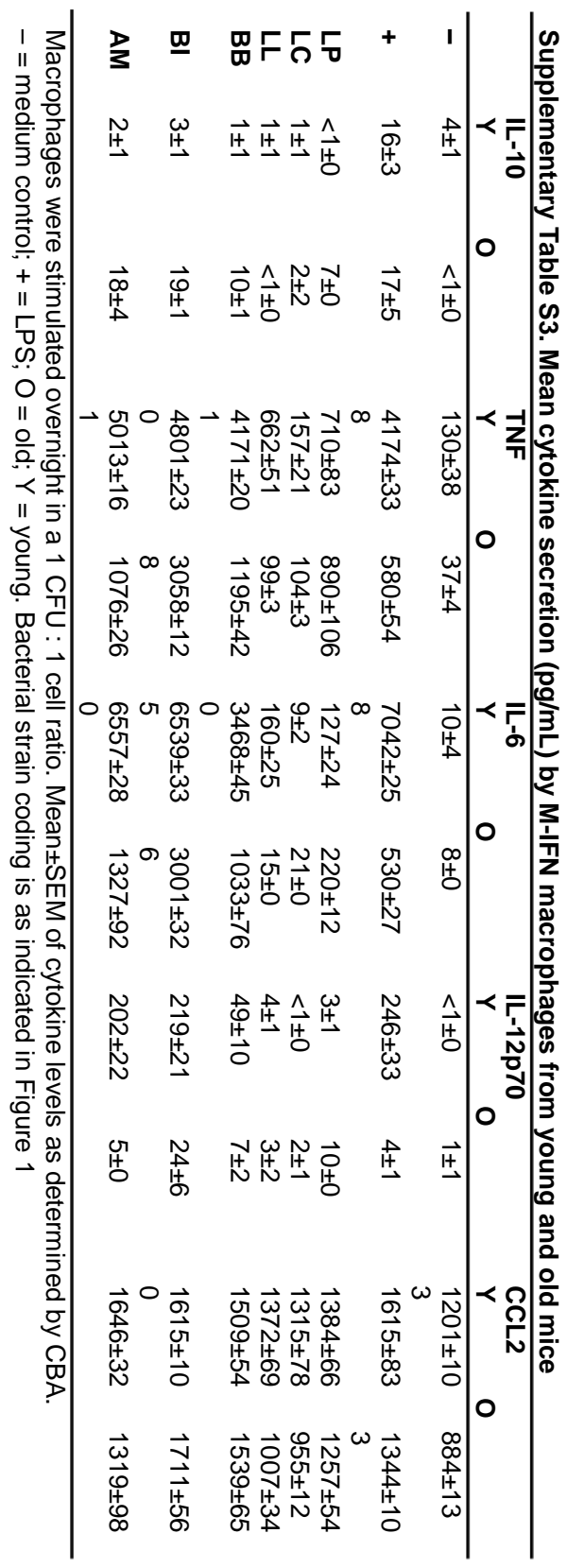


Effect of age on interaction between immune cells and bacterial strains in vitro 


\section{Chapter 6}

\section{Supplementation with Lactobacillus plantarum WCFS1 prevents age-related decline of mucus barrier in colon of accelerated aging $\operatorname{Ercc1}^{-/ \Delta 7}$ mice}

Adriaan A. van Beek ${ }^{1,2,3}$, Bruno Sovran ${ }^{2,4}$, Floor Hugenholtz ${ }^{2,5}$, Ben Meijer ${ }^{1}$, Joanne A. Hoogerland ${ }^{1}$, Violeta Mihailova ${ }^{1}$, Corine van der Ploeg ${ }^{1}$, Clara Belzer ${ }^{2,5}$, Mark V. Boekschoten $^{2,6}$, Jan J.H. Hoeijmakers ${ }^{7}$, Wilbert P. Vermeij ${ }^{7}$, Paul de $\operatorname{Vos}^{2,8}$, Jerry M. Wells $^{2,4}$, Pieter J.M. Leenen ${ }^{9}$, Rudi W. Hendriks ${ }^{10}$, Huub F.J. Savelkoul ${ }^{1,2}$

${ }^{1}$ Cell Biology and Immunology Group, Wageningen University, Wageningen, The Netherlands; ${ }^{2}$ Top Institute Food and Nutrition, Wageningen, The Netherlands; ${ }^{3}$ Gut Health and Food Safety, Institute of Food Research, Norwich, United Kingdom; ${ }^{4}$ Host Microbe Interactomics; ${ }^{5}$ Laboratory of Microbiology; ${ }^{6}$ Human Nutrition, Wageningen University, Wageningen, The Netherlands; ${ }^{7}$ Department of Molecular Genetics; ${ }^{9}$ Department of Immunology; ${ }^{10}$ Department of Pulmonary Medicine, Erasmus University Medical Center, Rotterdam, The Netherlands; ${ }^{8}$ University of Groningen, Groningen, The Netherlands. 
Chapter 6

\section{ABSTRACT}

Although it is clear that probiotics improve intestinal barrier function, little is known about the effects of probiotics on the aging intestine. We investigated effects of 10 -wk bacterial supplementation of Lactobacillus plantarum WCFS1, Lactobacillus casei BL23, or Bifidobacterium breve DSM20213 on gut barrier and immunity in 16-week-old accelerated aging $E r c c 1^{-/ \Delta 7}$ mice, which have a median lifespan of $\sim 20 \mathrm{wk}$, and their wild-type littermates.

The colonic barrier in Ercc $1^{-/ \Delta 7}$ mice was characterized by a thin $(<10 \mu \mathrm{m})$ mucus layer. $L$. plantarum prevented this decline in mucus integrity in $E r c c 1^{-1 / 4}$ mice, whereas $B$. breve exacerbated it. Bacterial supplementations affected the expression of immunerelated genes, including Toll-like receptor 4 . Regulatory $T$ cell frequencies were increased in the mesenteric lymph nodes of $L$. plantarum- and L. casei-treated $E r c c 1^{-/ \Delta 7}$ mice. L. plantarum- and L. casei-treated Ercc1 ${ }^{-/ \Delta 7}$ mice showed increased specific antibody production in a T cell-dependent immune response in vivo. By contrast, the effects of bacterial supplementation on wild-type control mice were negligible.

Thus, supplementation with L. plantarum - but not with L. casei and B. breve - prevented the decline in the mucus barrier in $E r c c 1^{-1 \Delta 7}$ mice. Our data indicate that age is an important factor influencing beneficial or detrimental effects of candidate probiotics. These findings also highlight the need for caution in translating beneficial effects of probiotics observed in young animals or humans to the elderly. 
Supplementation with L. plantarum WCFS1 prevents decline of mucus barrier in colon of $E r c c 1^{-1 \Delta 7}$ mice

\section{INTRODUCTION}

Aging is accompanied by multiple age-related diseases [17], posing a major burden to public health care [14]. With age, a decline in the regenerative potential of tissues due to stem cell exhaustion occurs [11]. Turnover in epithelial cells is rapid, and mounting evidence indicates that intestinal stem cells are compromised with aging [486]. For example, a crucial component of the intestinal barrier is mucus secreted by goblet cells [135]. The Muc2 glycoprotein regulates immunity by inducing tolerogenic signals in mucosal dendritic cells [138] and is important in host-microbe interactions [487]. Thus, changes in mucus quantity and integrity influence immunity $[137,138]$.

Aging is accompanied by the development of a low-grade inflammation ('inflammaging'), which is characterized by elevated IL-6 and TNF serum levels in elderly [71]. Involution of the thymus and the bone marrow (BM) leads to decreased $T$ and $B$ cell production [55, 488]. In contrast, the production of myeloid cells is enhanced with aging, characterized by a progressive increase of neutrophil frequencies in the circulation [73].

Probiotics are defined as live bacteria that confer health benefits to the host, for example by competing with pathogens, enhancing intestinal barrier function, and regulating immunity [163, 164]. They might therefore prevent some of the undesired agerelated intestinal barrier and immune effects. Probiotic supplementation of elderly subjects led to changes in fecal microbiota composition [489-491], and affected the distribution and function of NK cells, macrophages, granulocytes, and T cells in the circulation [492, 493]. Supplementation of aged mice with Lactobacillus paracasei resulted in increased IgG2a serum titers after antigenic challenge [494]. Middle-aged mice that were supplemented with Bifidobacterium animalis showed decreased colon permeability, extended lifespan, and improved quality of life [165]. Besides these studies, little is known about how exposure to probiotics impacts on the aging intestinal barrier and immune system. Moreover, it is unknown whether the beneficial effects of probiotics are age-dependent.

In this report, we have used an accelerated aging mouse model to evaluate the effects of candidate probiotics in aging. Based on a variety of histological, functional, metabolomic, and proteomic data, it has been concluded that $\operatorname{Ercc}^{-/ \Delta 7}$ mice resemble normal murine aging [495]. Recently, we have shown that the immune system of $E r c c 1^{-1 / 7}$ mice resembles the immune system of aged WT mice. For instance, we showed a similar decrease in B cell precursors and naïve $T$ cells, and a similar increase in memory T cells and regulatory T cells [496]. The ERCC1 protein is involved in multiple DNA repair pathways. Ercc1 ${ }^{-1 \Delta 7}$ mice (median lifespan $\sim 20$ weeks) are deficient for fully functional ERCC1 protein. The expression of ERCC1-XPF (excision repair crosscomplementation group 1-xeroderma pigmentosum group F) DNA repair endonuclease is reduced to $\sim 5 \%$ compared with $\mathrm{ErCC}^{+/+}$mice. Moreover, the residual ERCC1- 
$\mathrm{XPF}$ protein present is expressed from a truncated allele, and lacks the last 7 amino acids. A reduction of ERCC1 protein activity leads to increased accumulation of DNA damage, and hence results in an accelerated aging phenotype [60, 62].

The aim of this study was to investigate the potential of supplementation with candidate probiotic strains to ameliorate the effects of aging on the intestinal barrier and the immune system. Previously, probiotic activity was documented for Lactobacillus plantarum WCFS1 [453, 456, 457], Lactobacillus casei BL23 [442, 444], and relatives of Bifidobacterium breve DSM20213 [446]. We selected these strains on the basis of induced IL-10/TNF ratios in young and aged immune cells in vitro [497]. The three strains can be classified as potential pro-inflammatory ( $L$. plantarum), regulatory ( $L$. casel), or anti-inflammatory (B. breve), based on low, intermediate, or high IL-10/TNF ratios, respectively.

For this study, we supplemented 6-week-old $E r c c 1^{+/+}$mice and $E r c c 1^{-/ \Delta 7}$ mice with $L$. plantarum, L. casei, or B. breve for 10 weeks. Mucus barrier, microbiota composition, and gene regulation in the colon were analyzed, as well as the distribution of immune cells in various mucosal and peripheral lymphoid organs. We determined immune competence by antigenic challenge.

\section{MATERIALS AND METHODS}

\section{Mice}

The generation and characterization of $\operatorname{Ercc1}^{+/ \Delta 7}$ and $\operatorname{Erccl}^{-/+}$mice has been previously described [60]. Ercc1 $1^{-/ \Delta 7}$ mice were obtained by crossing $E r c c 1^{+/ \Delta 7}$ with $E r c c 1^{-/ t}$ mice of pure C57BI6/J and FVB backgrounds to yield $\mathrm{ErCC1}^{-1 / \Delta 7}$ with an F1 C57BI6J/FVB hybrid background. Genotyping was performed as described previously [498]. Wildtype littermates (C57BI/6xFVB F1) were used as controls. Four-month-old and 18month-old C57BI6/J mice were purchased from Harlan (Horst, The Netherlands; only used in Figure 1).

Animals were housed in individual ventilated cages under SPF conditions. Experiments were performed in accordance with the Principles of Laboratory Animal Care and with Dutch legislation. This study was carried out in accordance with the recommendations of the Dutch Ethical Committee of Wageningen that approved the work. Blood was taken from mice being sacrificed, and serum was frozen in $-80^{\circ} \mathrm{C}$ for later use. After mice $(n=4-6)$ were sacrificed, feces from colon was collected and snapfrozen. Distal ileum and proximal colon sections were isolated and fixed in Carnoy or snap-frozen in liquid nitrogen. Bone marrow, thymus, spleen, mesenteric lymph nodes, and Peyer's patches were isolated. 
Supplementation with L. plantarum WCFS1 prevents decline of mucus barrier in colon of $\operatorname{Ercc} 1^{-1 \Delta 7}$ mice

\section{Bacterial cultures and supplementation}

Lactobacillus plantarum WCFS1, Lactobacillus casei BL23, and Bifidobacterium breve DSM20213 were grown on MRS medium (Merck, Darmstadt, Germany) until stationary phase, frozen in glycerol, and stored in $-80^{\circ} \mathrm{C}$ until use. Upon use, bacteria were thawed and 10x diluted in NaHCO3/PBS buffer. Around $2 * 10^{8} \mathrm{CFU}$ in $200 \mu \mathrm{L}$ were administered to mice by gavage, three times per week. Treatment of mice started at 6 weeks of age until one day before sacrifice at 16 weeks or until death.

\section{Histology and fluorescence in situ hybridization (FISH)}

Carnoy-fixed proximal colon sections were embedded in paraffin. Paraffin sections (5 $\mu \mathrm{m})$ were attached to poly-L-lysine-coated glass slides (Thermo Scientific, Germany). After overnight incubation at $37^{\circ} \mathrm{C}$, slides were de-waxed and rehydrated. Sections were stained with hematoxylin and eosin (H\&E) and PAS/Alcian blue. Mucus layer thickness was measured using ImageJ software ( $\mathrm{NIH}$, Maryland, USA), as previously published [499]. For detection of bacteria, tissue sections were used for FISH, as previously published [137].

\section{MIT-Chips/16S Sequencing}

Microbiota composition in colonic content was analyzed by Mouse Intestinal Tract Chip (MITChip), as described previously [500]. The data were normalized and analyzed using a set of R-based scripts in combination with a custom-designed relational database, which operates under the MySQL database management system. For the microbial profiling, the Robust Probabilistic Averaging signal intensities of 2667 specific probes for the 94 genus-level bacterial groups detected on the MITChip were used [501]. Diversity calculations were performed using a microbiome R-script package (https://github.com/microbiome). Multivariate statistics, redundancy analysis, and principal response curves were performed in Canoco 5.0 and visualized in triplots or a principal response curves plot [502].

\section{RNA isolation and transcriptome analysis}

Total RNA was isolated from proximal colon ( $n=3-6$ per group) using the RNeasy kit (Qiagen) with a DNase digestion step according to the manufacturer's protocol. Transcriptome analysis on individual samples was performed as previously described [137]. 
Chapter 6

\section{General flow cytometry procedures}

Single-cell suspensions of bone marrow (BM) were obtained by crushing femurs, tibias, iliac crests, and sternum with mortar and pestle. BM cells were then filtered on a $40 \mu \mathrm{m}$ cell strainer. A proportion of the BM cells was frozen for later use in in vitro cultures. Spleen, mesenteric lymph nodes (MLN), Peyer's patch (PP), thymus and peritoneal cavity single-cell suspensions were obtained by gently pushing cells through a $40 \mu \mathrm{m}$ cell strainer with a syringe. All cells were stained for extracellular markers and dead cells were identified with fixable live/dead stain (Ebioscience, San Diego, CA, USA), after which intracellular staining was enabled by fixing and permeabilizing cells with Fix/Perm buffer (Ebioscience) according to manufacturer's instructions. Antibodies used for flow cytometric measurements are listed in Supplementary Table 1. All flow cytometric measurements were performed on a Canto II flow cytometer (BD Biosciences, Erembodegem, Belgium). FlowJo vX.07 software (Tree Star) was used for data analysis. Gating of all presented immune cell populations was based on single live cells.

\section{Spleen cell cultures}

Splenic cells were cultured at $10^{6}$ cells $/ \mathrm{mL}$ for four days in the absence or presence of $5 \mu \mathrm{g} / \mathrm{mL}$ concanavalin A (ConA). Proliferation was measured by Ki-67 (Ebioscience). Supernatants were stored at $-20^{\circ} \mathrm{C}$. After thawing, levels of IL-2, IL-4, IL-6, IL-10, IL17A, IFN- $\gamma$, and TNF were measured with the Cytometric Bead Array Th1/Th2/Th17 Kit (BD Biosciences), according to manufacturer's instructions. Samples were acquired on a Canto II flow cytometer. Data were analyzed using FCAP Array version 3.0 (BD Biosciences) software.

\section{Antibody titers in serum}

Levels of $\lg M, \lg G 1, \lg G 2 a, \lg G 2 b, \lg G 3, \lg E$, and $\lg A$ were analyzed in serum using ProcartaPlex Mouse Antibody Isotyping Panel kit on the Luminex platform (Affymetrix, Santa Clara, CA, USA) according to the manufacturer's instructions. Data were acquired on a BioPlex 200 (Bio-Rad, Hercules, CA, USA) and analyzed with BioPlex software (version 5.0, Bio-Rad).

\section{In vivo immunization and antibody detection}

Primary and secondary T-cell dependent (TD) immune responses against TNP-KLH were measured 7 days after primary i.p. immunization and 7 days after i.p. booster immunization. The primary immunization was performed at 8 weeks of age (TNP-KLH in alum), booster doses were injected at 12 weeks of age (TNP-KLH in PBS). Total and TNP-specific Ig subclasses were determined by sandwich ELISA as previously described [503]. 
Supplementation with L. plantarum WCFS1 prevents decline of mucus barrier in colon of $E r c c 1^{-1 \Delta 7}$ mice

\section{Statistical analysis}

Values are expressed as mean + SEM. Normal distribution of the data was confirmed using the Kolmogorov-Smirnov test. Statistical comparisons were performed using the two-sided Student's $t$ test. Where non-Gaussian distribution was demonstrated, we applied the nonparametric Mann-Whitney $U$ test. Where no equal variances were observed, we applied the two-sided Student's t test with Welch's correction. Statistical comparisons for lifespan data were performed using the log-rank (Mantel-Cox) test. Statistical comparisons for serum immunoglobulins were performed using two-way ANOVA, with subsequent Bonferroni posttests. Values of $p<0.05$ were considered to be statistically significant. Values between $p>0.05$ and $p<0.10$ were considered as a trend.

\section{RESULTS}

\section{The mucus layer in the colon declines with age}

To assess the mucus barrier in normal and accelerated aging, we compared the proximal colon of 4-month-old (young) with 18-month-old (aged) WT mice, and of 6-weekold (young) with 16-week-old (aged) Ercc $1^{-1 \Delta 7}$ mice. We observed that in aged WT and $E_{\text {rcc1 }}{ }^{-1 \Delta 7}$ mice, a thinner mucus layer was present, compared with young WT and $\operatorname{Ercc1}^{-1 / \Delta 7}$ mice (Figure 1A). With ImageJ, we measured the thickness of the mucus layer. In young WT and $E r c c 1^{-1 \Delta 7}$ mice, a mucus layer of around $20 \mu \mathrm{m}$ was present, whereas in normal and accelerated aged mice, a significantly thinner mucus layer of less than $10 \mu \mathrm{m}$ was observed $(p<0.001$; Figure 1B).

\section{Bacterial supplementations do not change the mucus layer in colon of young $\mathrm{ErCc1}^{+/+}$mice}

To determine the effects of the three selected bacterial strains in the young intestine, we analyzed proximal colon tissues of 10 -wk treated $\mathrm{ErCC}^{+/+}$mice. No change in tissue integrity (H\&E) or mucus layer (PAS/Alcian Blue) was observed in the colon after supplementation with bacterial strains (Figure 1C).

\section{Age-related decline in the mucus barrier is prevented by supplementation of Ercc1 $^{-/ \Delta 7}$ mice with L. plantarum}

Because the mucus layer declines with age, we questioned whether bacterial supplementation of $\mathrm{ErCC}^{-1 \Delta 7}$ mice prevents the decline in mucus barrier. Colon tissue of 10wk treated $E r c c 1^{-1 / 7}$ mice was checked for tissue integrity and mucus layer. In contrast to our findings in $\mathrm{ErcC1}^{+/+}$mice, bacterial supplementation had significant effects on tissue integrity and the mucus layer. In Ercc ${ }^{-1 \Delta 7}$ mice supplemented with L. plantarum, the colon showed a thicker mucus layer than in their controls (Figure 1D). On the con- 
trary, $E r c c 1^{-1 / \Delta 7}$ mice supplemented with $L$. casei or $B$. breve showed loss of tissue integrity, and in the case of $B$. breve supplementation also a deteriorated mucus layer. L. plantarum supplementation prevented age-related decline in the mucus layer compared with controls $(p<0.001$; Figure $1 \mathrm{E})$, with a mucus thickness comparable to young $\mathrm{ErcC1}^{+/+}$mice. No difference in mucus thickness was observed after supplementation with $L$. casei. A significant loss in mucus thickness, however, was observed after supplementation with $B$. breve $(p<0.001)$. Treatment with $L$. plantarum, in contrast to $L$. casei and $B$. breve, resulted in improved spatial compartmentalization of bacteria in the colon of $\operatorname{Ercc}^{-1 / 4}$ mice (Figure $1 \mathrm{~F}$ ).

Collectively, these data show that $L$. plantarum supplementation improves the mucus layer in the aged (but not young) colon. In addition, supplementation with $L$. casei or $B$. breve exacerbates the age-related decline of mucus barrier in the colon.

\section{Bacterial supplementation associated with minor alterations in colonic microbi- ota composition}

As we introduced bacteria by bacterial supplementations into the intestinal microbial community, we investigate whether changes in the microbiota composition were underlying the observed changes in the mucus barrier of $\operatorname{Ercc}^{-/ \Delta 7}$ mice. Microbiota composition was determined by performing 16S rRNA gene microbiota profiles of feces. The bacterial supplementations did not significantly alter microbial diversity nor richness (data not shown).

Redundancy analysis (RDA) showed that $10.1 \%$ of the total variability of the gut microbiota can be related to the bacterial supplementations (Figure 2). No statistical significance was established. The first ordination axis explained $4.9 \%$ of the variability and separated $E r c c 1^{-1 \Delta 7}$ mice supplemented with either of the three bacterial strains from the control $\operatorname{Ercc}^{-/ \Delta 7}$ mice. The second ordination axis explained $3.6 \%$ of the variability but did not result in a separation between groups. The third ordination axis explained an additional $1.6 \%$ of the variability (data not shown).

To assess whether significant changes in the microbial genus-like bacterial groups existed after different bacterial supplementations, we performed the Wilcoxon test. Subdoligranulum was higher in mice supplemented with $L$. casei $(p<0.05)$, whereas it 


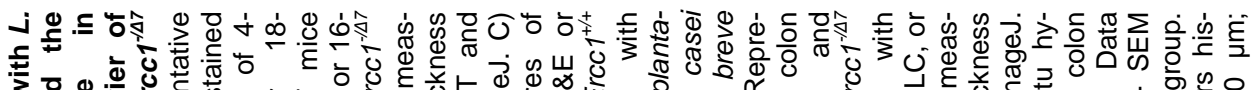

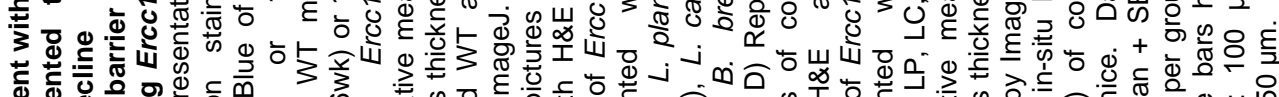

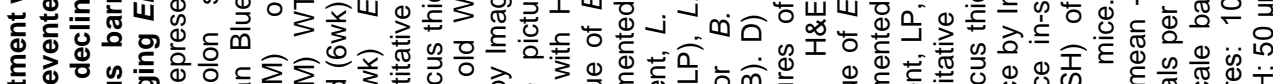

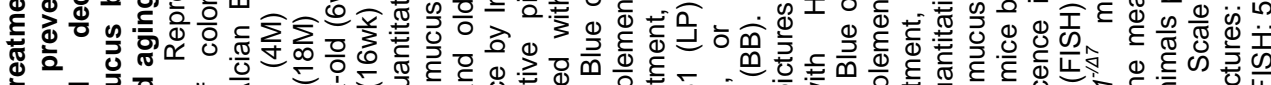

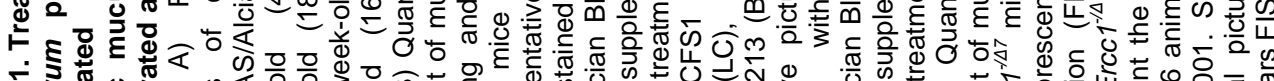

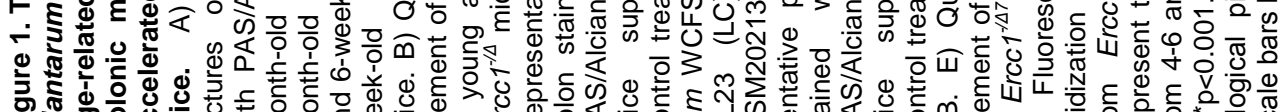

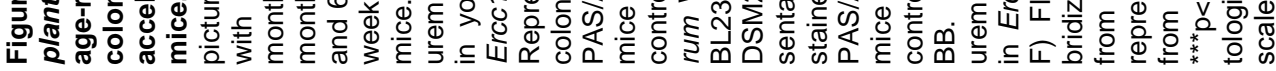

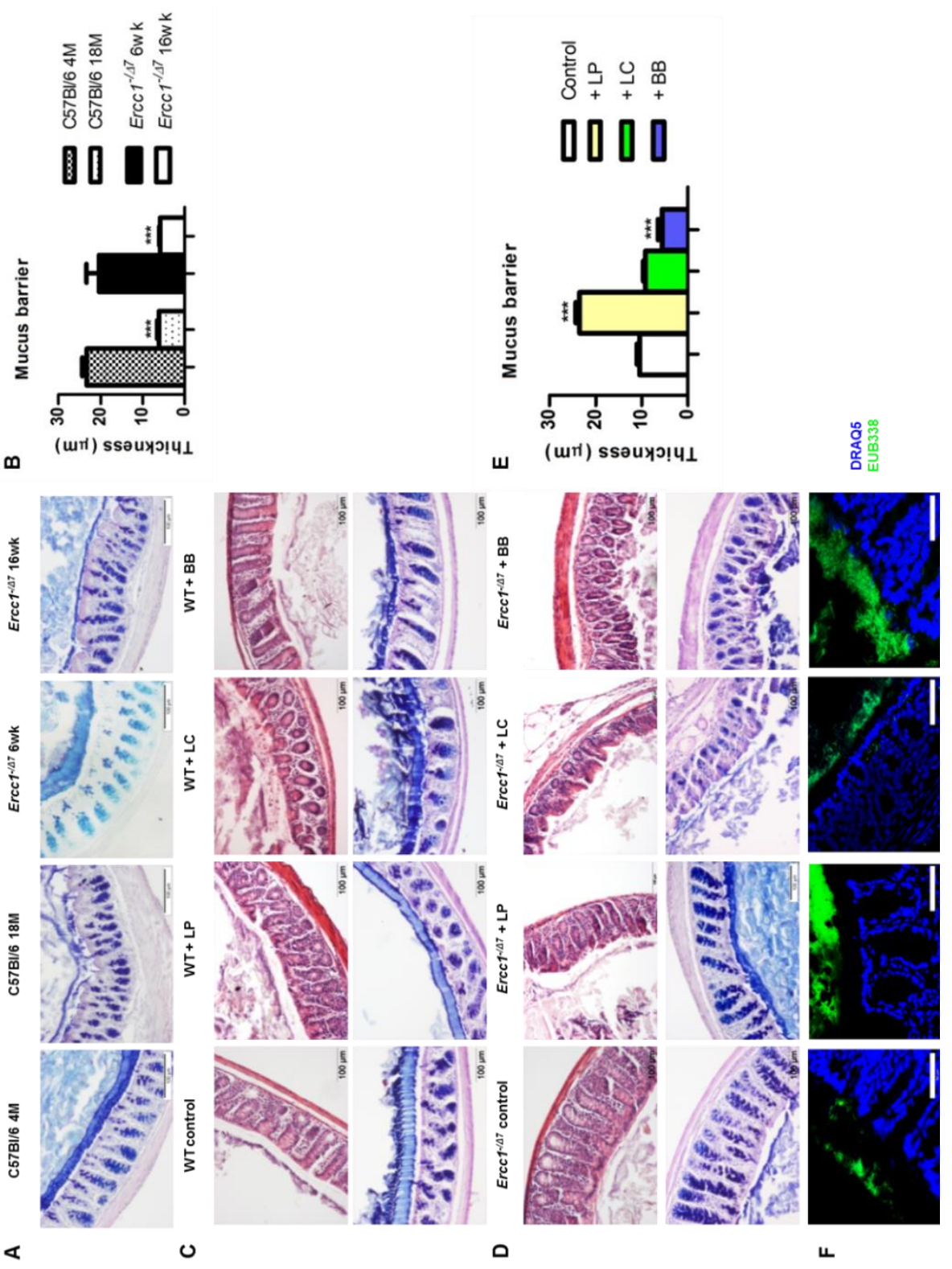




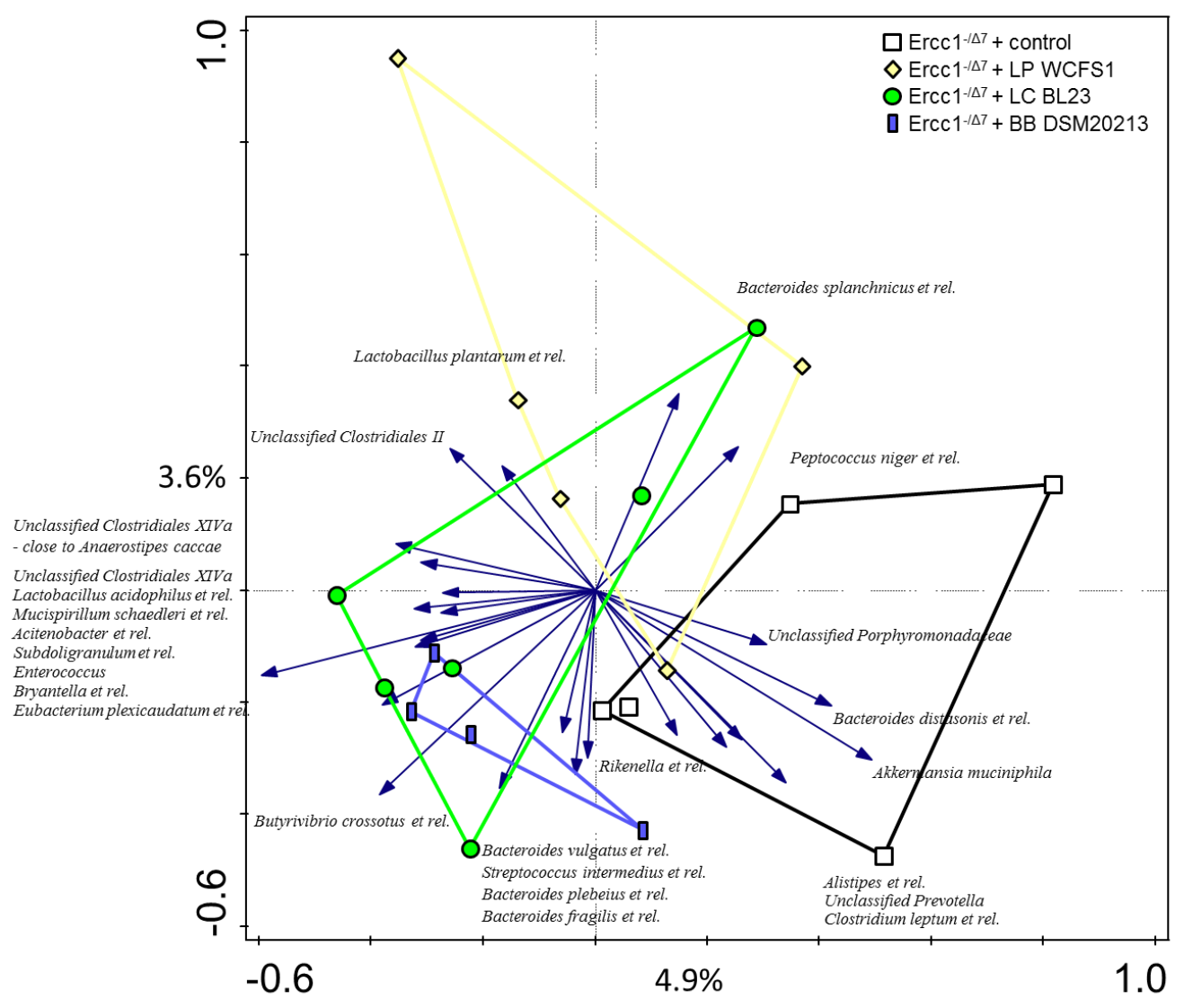

Figure 2. The effect of bacterial supplementations on colonic microbiota composition in Ercc1 $1^{-/ \Delta 7}$ mice. Redundancy analysis of the microbial composition after bacterial supplementations, on genus-like level of the MITChip analysis. Mice belonging to control-, LP-, LC-, and BB-treated groups are indicated by white squares, yellow diamonds, green circles, and blue rectangles, respectively. First and second ordination axes are plotted, showing $4.9 \%$ and $3.6 \%$ of the variability in the dataset, respectively. No significant changes were observed. $\mathrm{LP}=$ L. plantarum $\mathrm{WCFS1} ; \mathrm{LC}=$ L. casei $\mathrm{BL23} ; \mathrm{BB}=$ B. breve DSM20213.

tended to be higher in mice supplemented with $B$. breve $(p=0.05)$, as compared with control mice (Figure 3). Akkermansia muciniphila tended to be less present $(p=0.06)$ in mice supplemented with L. plantarum compared with control mice. Eubacterium plexicaudatum and a close relative to Anaerostipes caccae tended to be higher $(p=0.06)$ in $\operatorname{Ercc1}^{-1 / 47}$ mice supplemented with $L$. casei.

These data suggest that colonic microbial differences between control-treated Ercc1 ${ }^{1 \Delta 7}$ mice and $\operatorname{Ercc}^{-1 / \Delta 7}$ mice treated with bacterial supplementations do not explain observed changes in colon. 
Supplementation with L. plantarum WCFS1 prevents decline of mucus barrier in colon of $E r c c 1^{-1 / \Delta 7}$ mice

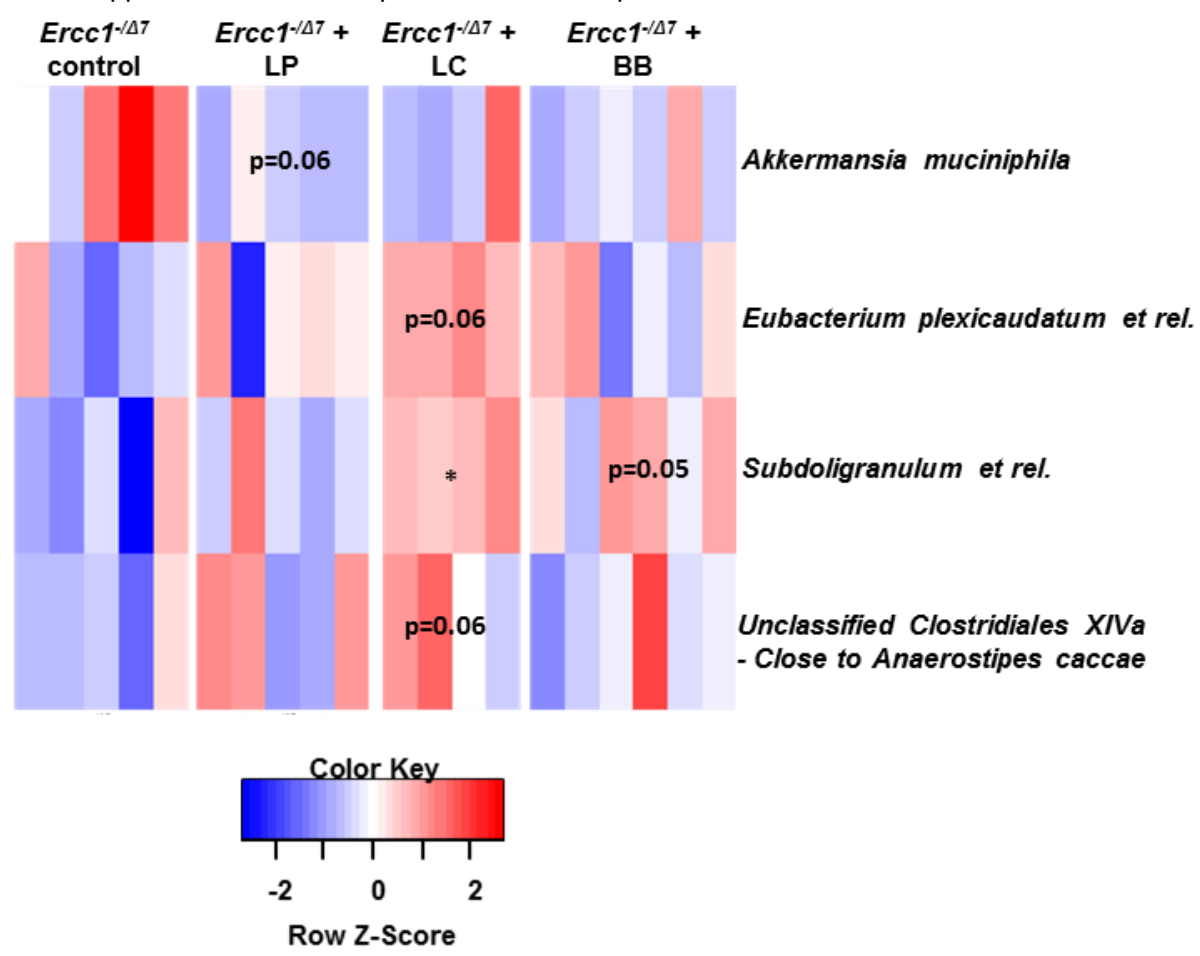

Figure 3. Bacterial supplementation induced changes in bacterial taxa in the colon of Ercc $1^{-1 \Delta 7}$ mice. Wilcoxon tests comparing mice treated with bacterial strains with control group. Data represent $n=4-6$ mice per group. ${ }^{*}=p<0.05$.

\section{Distinct gene expression profiles in colon after each bacterial supplementation}

To understand the mechanisms by which bacterial supplementation changes the mucus barrier, we performed transcriptome analysis on the proximal colon of $E r c c 1^{-1 / 4}$ mice. Gene expression microarrays revealed relatively low numbers of regulated genes: 84 by L. plantarum, 238 by L. casei, and 384 by B. breve. Only a few genes were overlapping between two or three different bacterial supplementations, whereas most of the regulated genes were distinctly regulated by one of the treatments (Figure 4).

Several growth- and immune-related genes were differentially expressed after bacterial supplementation. Apolipoprotein (APO) A-1, APOA-4, suppressor of cytokine signaling (SOCS) 3, and Toll-like receptor (TLR) 4 were upregulated more than 1.2-fold after L. plantarum supplementation (data not shown). Several immunoglobulin variable genes and TLR13 were upregulated after administration of $L$. casei, whereas defensin $40 \beta$ was 1.3-fold downregulated. Defensin $24 \alpha$, amphiregulin, and keratinocyte growth factor 7 (FGF7) were upregulated more than 1.4-fold after administration of $B$. breve, while TLR6, TLR7, and CCL3 (MIP-1 $\alpha$ ) were more than 1.2-fold downregulated (data not shown). 


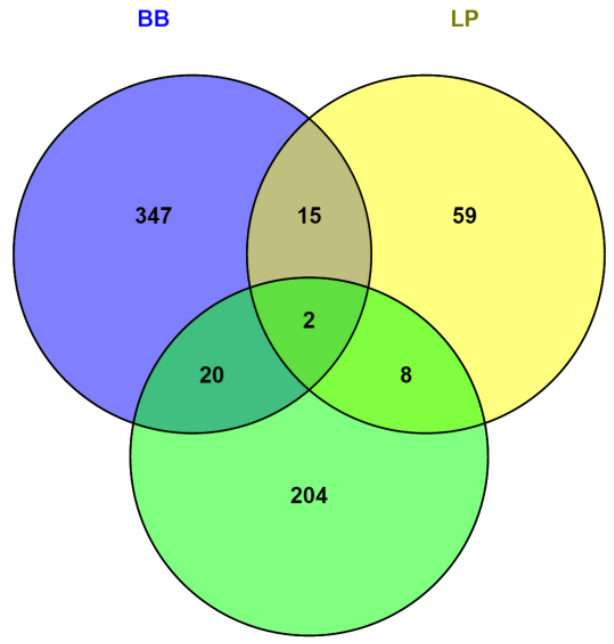

LC
Figure 4. Venn diagrams of differentially regulated genes in colon of Ercc $^{-/ \Delta 7}$ mice after bacterial supplementations. Total number of genes altered in the proximal colon of $E r c c 1^{-1 / \Delta 7}$ mice treated with $L$. plantarum WCFS1 (LP), L. casei BL23 (LC), or B. breve DSM20213 (BB) compared with control-treated Ercc ${ }^{-1 / 47}$ mice. Venn diagram of the total number of genes up-regulated and down-regulated in the proximal colon of Ercc $1^{-1 / 47}$ mice treated with LP, BB, or LC $(p<0.05$ and $>1.2$-fold difference).

\section{Bacterial supplementation alters growth- and immune-related pathways in colon}

We applied a gene set enrichment analysis (GSEA)[504] to gain insight into the regulated pathways by bacterial supplementations. GSEA revealed that $L$. plantarum and L. casei supplementation enhanced several processes involved in growth and cell cycle, and immunity (Supplementary Table 2), whereas $B$. breve supplementation inhibited several processes in immunity (Supplementary Table 3). Of note, supplementation with $L$. casei and $B$. breve enhanced the processes involved in the unfolded protein response (UPR). Moreover, L. plantarum enhanced DNA repair pathways.

Upstream regulators that can explain the observed changes in gene expression were identified using Ingenuity Upstream Regulator Analysis. Upstream regulators predicted to be activated or inhibited upon bacterial supplementation are listed in Table 1. Several growth factors were activated after $L$. plantarum supplementation: leptin, epidermal growth factor (EGF), platelet-derived growth factor (PDGF) BB, early growth response protein (EGR) 1, and insulin-like growth factor (IGF) 1. Resistin-like $\beta$ (RTNLB) was activated in colon of mice supplemented with $L$. casei, whereas GATA3 was inhibited. B. breve supplementation inhibited fibroblast growth factor (FGF) 2. Inflammatory cytokines (IFN- $y$, IL-1 $\beta$, IL-4, TNF) and CD40L (CD154) were activated in colon of mice supplemented with $L$. plantarum, compared with colon of mice supplemented with control. In contrast, IFN-y, IgG, GATA2, and ITK were inhibited in colon of mice supplemented with $B$. breve, EGF, insulin, and platelet-derived growth factor (PDGF) $\mathrm{BB}$ were activated by both $L$. plantarum and $B$. breve supplementation. 
Supplementation with L. plantarum WCFS1 prevents decline of mucus barrier in colon of $E r c c 1^{-1 \Delta 7}$ mice

Table 1. Activation z-scores of upstream regulators in proximal colon of $\mathrm{Ercc1}^{-/ \Delta 7} \mathrm{mice}$ after bacterial supplementations L. plantarum WCFS1 (LP), L. casei BL23 (LC), or B. breve DSM20213 (BB) as determined by Ingenuity. Upstream regulators involved in immunity are highlighted in blue; upstream regulators involved in growth and cell cycle are highlighted in orange. Cut-off values for activation $z$-score $\geq 1.5$ or $\leq-1.5$ combined with $p<0.05$. Activated in blue, inhibited in red.

\begin{tabular}{|c|c|c|c|}
\hline Upstream Regulator & LP & LC & BB \\
\hline Leptin & 2.41 & & \\
\hline EGF & 2.36 & & 3.36 \\
\hline IL4 & 2.18 & & \\
\hline IFN- $\nu$ & 2.00 & & -1.35 \\
\hline PDGF BB & 2.00 & & 1.15 \\
\hline P38 MAPK & 1.97 & & \\
\hline CD40L & 1.96 & & \\
\hline palmitic acid & 1.96 & & \\
\hline EGR1 & 1.95 & & \\
\hline IGF1 & 1.82 & & \\
\hline IL1 $1 \beta$ & 1.77 & & \\
\hline ethanol & 1.76 & & \\
\hline CREB1 & 1.55 & & \\
\hline CREBBP & 1.54 & & \\
\hline TNF & 1.53 & & \\
\hline KLF4 & & 2.04 & \\
\hline Resistin-like $\beta$ & & 2.00 & \\
\hline PML & & -1.73 & \\
\hline miR-4800-5p & & -1.98 & \\
\hline GATA3 & & -1.98 & \\
\hline MTOR & & -2.00 & \\
\hline $\operatorname{miR}-4455$ & & -2.22 & \\
\hline ADCYAP1 & & & 2.60 \\
\hline EDN1 & & & 2.17 \\
\hline WNT3A & & & 2.16 \\
\hline VIP & & & 1.95 \\
\hline FGF2 & & & 1.74 \\
\hline GLI1 & & & 1.63 \\
\hline miR-6967-5p & & & -1.58 \\
\hline Klra7 (includes others) & & & -1.87 \\
\hline $\lg G$ & & & -1.89 \\
\hline $\mathrm{EZH} 2$ & & & -1.96 \\
\hline GATA2 & & & -2.00 \\
\hline ANXA7 & & & -2.00 \\
\hline$m i R-4707-5 p$ & & & -2.16 \\
\hline ITK & & & -2.19 \\
\hline miR-4459 & & & -2.63 \\
\hline
\end{tabular}

ADCYAP $=$ adenylate cyclase activating polypeptide; $A N X=$ annexin CREB $(B P)=$ cAMP-responsive element (binding protein); EDN = endothelin; EGF = epidermal growth factor; $E G R=$ early growth response protein; $\mathrm{EHZ}=$ enhancer of zeste homolog; FGF = fibroblast growth factor; $\mathrm{GLI}$ = glioma-associated oncogene family zinc finger; IFN = interferon; IGF = insulin-like growth factor; ITK = IL-2-inducible T cell kinase; $\mathrm{KLF}=$ kruppel-like factor; KIra = killer cell lectin-like receptor, subfamily A; LEP = leptin; MTOR = mechanistic target of rapamycin; PDGF = platelet-derived growth factor; PML = promyelocytic leukemia protein; RETNLB = resistin-like $\beta$; TNF = tumor necrosis factor; VIP = vasoactive intestinal peptide; WNT = wingless-type MMTV integration site family. 
These data indicate that the immune system in the colon is regulated by bacterial supplementations.

\section{L. plantarum and $L$. casei supplementation induce regulatory $T$ cells in MLN}

Based on the regulation of immune genes by bacterial supplementations, we tested whether the distribution of immune cells was altered in mucosal immune organs of $\operatorname{ErCC1}^{-1 / 47}$ mice.

First, we evaluated changes in distribution of immune cells in Peyer's patches (PP) and mesenteric lymph nodes (MLN). B cell frequencies were reduced in PP and MLN $(p<0.05)$ after $L$. casei supplementation in $\operatorname{Ercc}^{-1 / \Delta 7}$ mice (Figure $\left.5 A\right)$. In contrast, frequencies of $T$ cells were increased in PP $(p<0.01)$ and $M L N(p<0.05$; Figure $5 B)$. The frequencies of regulatory $T$ (Treg) cells in MLN were increased after $L$. plantarum and L. casei supplementation $(p<0.05$; Figure $5 C, 5 D)$. No changes in distribution of $B$ and $\mathrm{T}$ cells was observed upon bacterial supplementation in $\mathrm{ErCC}^{+/+}$mice, except for a tendency to decreased Treg cells after $L$. casei supplementation ( $p=0.09$; Supplementary Figure 1).

A

B cells (PP)

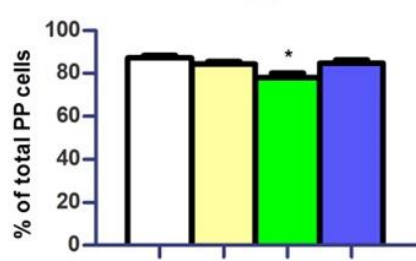

B

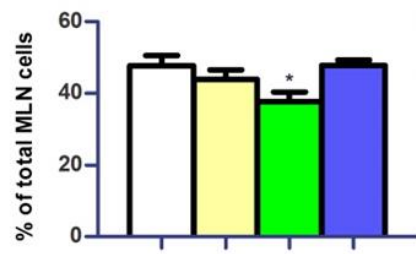

C

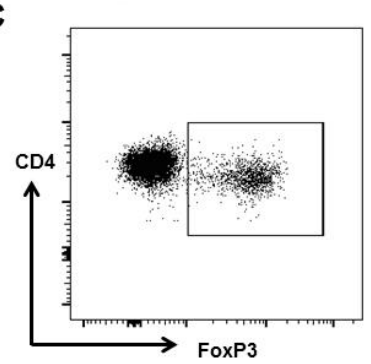

T cells (PP)

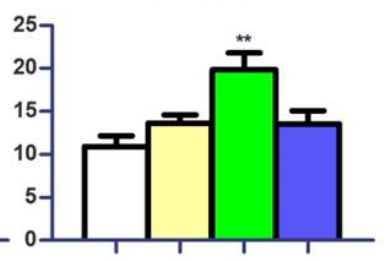

T cells (MLN)

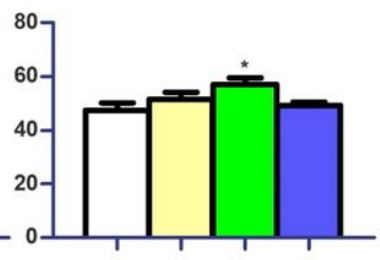

D

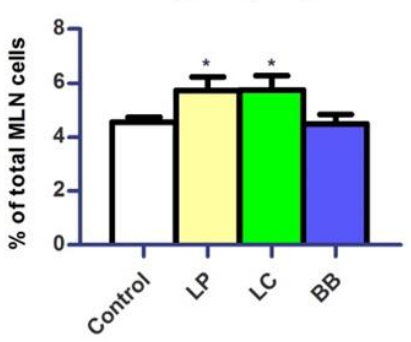

Figure 5. Distribution of B cells and $T$ cells in Peyer's patches (PP) and mesenteric lymph nodes (MLN) upon bacterial supplementation in $\operatorname{Ercc1}^{-/ \Delta 7}$ mice. $A / B$ ) Mean frequencies of $B$ and $T$ cells in PP and MLN were determined by flow cytometry. B cells were defined as $\mathrm{CD}^{+}{ }^{+}, \mathrm{T}$ cells were defined as $\mathrm{CD}^{+}$. C) Flow cytometric analysis of $\mathrm{CD}^{+} \mathrm{CD} 4^{+} \mathrm{CD} 8^{-}$regulatory $\mathrm{T}$ (Treg) cells in MLN. D) Mean frequencies of Treg cells in MLN. Data represent the mean + S.E.M. from 4-6 animals per group. $\mathrm{LP}=L$. plantarum WCFS1; LC = L. casei BL23; BB $=B$. breve DSM20213. ${ }^{*}=p<0.05$; ${ }^{* *}=\mathrm{p}<0.01$. 


\section{L. casei raises systemic inflammatory markers}

Next, we assessed distribution of immune cells in the spleen. First, we noted that the relative spleen weight increased after $L$. casei supplementation in $E r c c 1^{-1 \Delta 7}$ mice (Supplementary Figure 2A). Splenic B cell frequencies tended to be decreased after $L$. casei supplementation ( $p=0.06$; Figure $6 A$ ), but no changes in $T$ cell frequencies were observed (Figure 6B). Treg cell frequencies in the spleen were increased after $L$. casei supplementation in $\operatorname{ErCc} 1^{-1 \Delta 7}$ mice $(\mathrm{p}<0.05$; Figure $6 \mathrm{C})$.

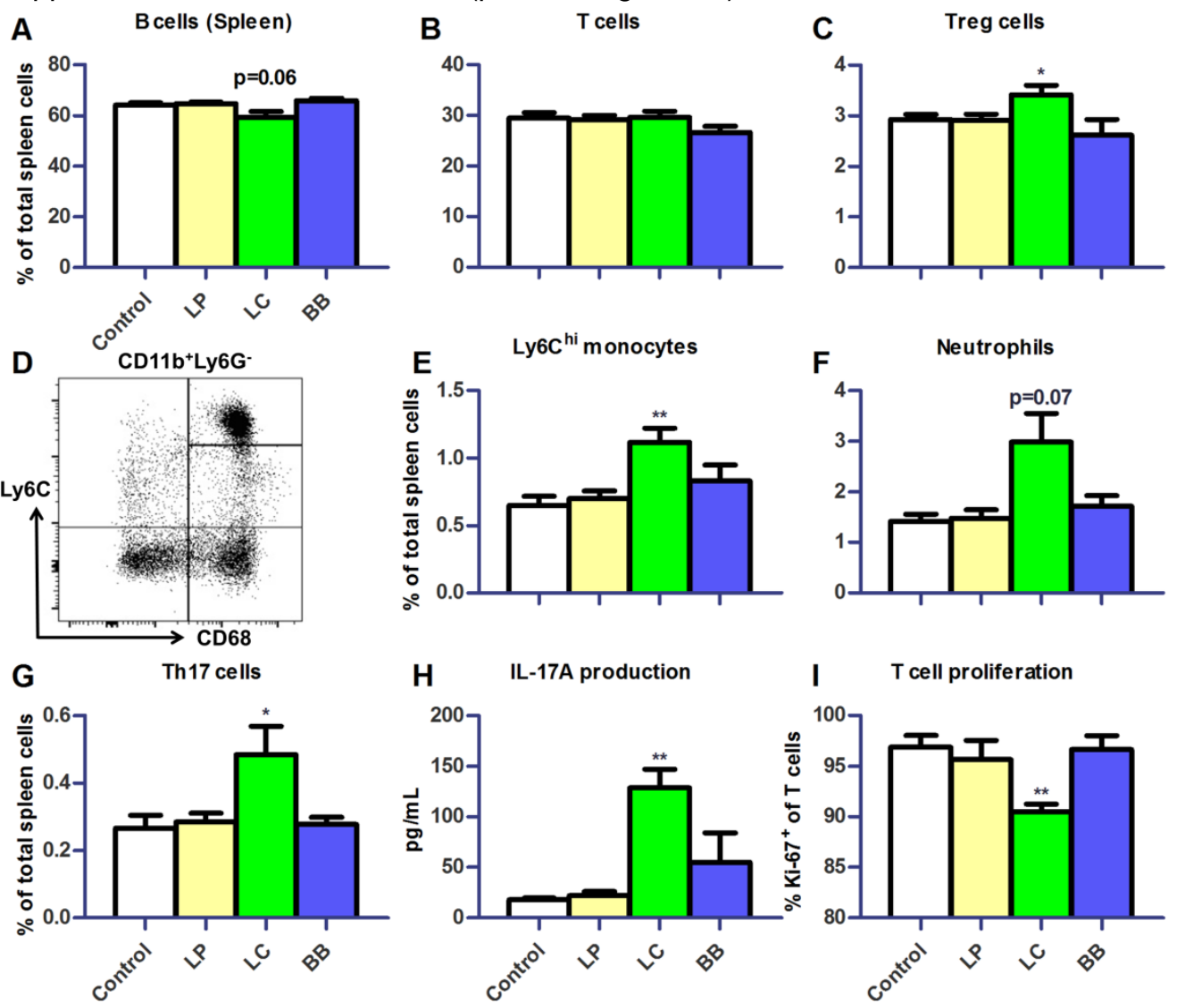

Figure 6. L. casei supplementation of $E r c c 1^{-1 / 47}$ mice raised inflammatory markers in spleen. A/B) Mean frequencies of $B$ and $T$ cells in spleen were determined by flow cytometry. $B$ cells were defined as $\mathrm{CD}_{19}{ }^{+}, \mathrm{T}$ cells were defined as $\mathrm{CD}^{+}$. C) Mean frequencies of Treg cells in spleen. D) Flow cytometric analysis of splenic monocytes. CD11 $\mathrm{b}^{+} \mathrm{Ly} 6 \mathrm{G}^{-} \mathrm{CD} 68^{+}$cells were divided in Ly6C $\mathrm{C}^{\text {hi }}$, Ly6C $\mathrm{C}^{\text {int }}$, and Ly6 $\mathrm{C}^{10}$ monocytes. E-G) Mean frequencies of Ly6C ${ }^{\text {hi }}$ monocytes, neutrophils, and CD3 ${ }^{+} C D 4^{+} C D 8$ Roryt $^{+}$Th17 cells were determined by flow cytometry. H) Mean concentration of IL-17A production by splenocytes stimulated with ConA for four days, as determined by Cytometric Bead Array. I) Mean proliferating T cells (Ki-67+) in splenocyte culture stimulated with ConA for four days, as determined by flow cytometry. Data represent the mean + S.E.M. from $4-6$ animals per group. ${ }^{*}=p<0.05 ;{ }^{* *}=p<0.01$. LP $=$ L. plantarum WCFS1; LC $=$ L. casei $\mathrm{BL} 23 ; \mathrm{BB}=$ B. breve DSM20213.

Increased frequencies of $C D 11 b^{+}$Ly6G $\mathrm{CD}^{-} 68^{+}$Ly6 $\mathrm{C}^{\text {hi }}$ monocytes $(\mathrm{p}<0.01$; Figure $6 \mathrm{D}$, $6 \mathrm{E})$ and a tendency to increased frequencies of $\mathrm{CD} 11 \mathrm{~b}^{+} \mathrm{CD} 68^{\text {int }} \mathrm{Ly} 6 \mathrm{C}^{\mathrm{int}} \mathrm{Ly}_{6 \mathrm{G}}{ }^{+}$neutrophils were observed after $L$. casei supplementation ( $p=0.07$; Figure $6 F$ ). In addition, 
$\mathrm{CD}^{+} \mathrm{CD} 4^{+} \mathrm{RORyt}^{+}$Th17 cells (Supplementary Figure 2B) were increased after L. casei supplementation $(p<0.05$; Figure $6 \mathrm{G})$. A four-day culture of splenocytes stimulated with concanavalin $A$ (ConA), showed increased IL-17A production $(p<0.01$; Figure $6 \mathrm{H})$ and decreased $T$ cell proliferation in splenocytes derived from $L$. casei-treated mice $(p<0.01$; Figure $6 \mathrm{I})$. None of these changes were observed in $\mathrm{Ercc}^{+/+}$mice treated with bacterial supplementations (Supplementary Figure 3).

\section{Immune cell development affected after Lactobacillus supplementation}

We subsequently investigated the development of $B$ cells and myeloid cells in bone marrow (BM) and of $\mathrm{T}$ cells in thymus of $E r c c 1^{-1 \Delta 7}$ mice, as the observed changes in cell distribution in PP, MLN, and spleen might be explained by an altered migration or production. In the $\mathrm{BM}$, we observed significantly higher $\operatorname{Lin}^{-} \mathrm{CD} 117^{\mathrm{hi}} \mathrm{CD} 11 \mathrm{C}^{-} \mathrm{CD} 135^{-}$ CD16/32 $2^{+}$granulocyte-monocyte precursor (GMP), CD11 b ${ }^{+} L y 6 G^{+}$neutrophil, and Ly6C ${ }^{\text {hi }}$ CD31- monocyte frequencies after $L$. casei supplementation (Figure 7A-7C). Frequencies of total $C D 19^{+} C D 45 R^{+} B$-lineage cells were decreased after $L$. plantarum $(p<0.05)$ and $L$. casei supplementation $(p<0.001)$, but not after $B$. breve supplementation (Figure 7D).
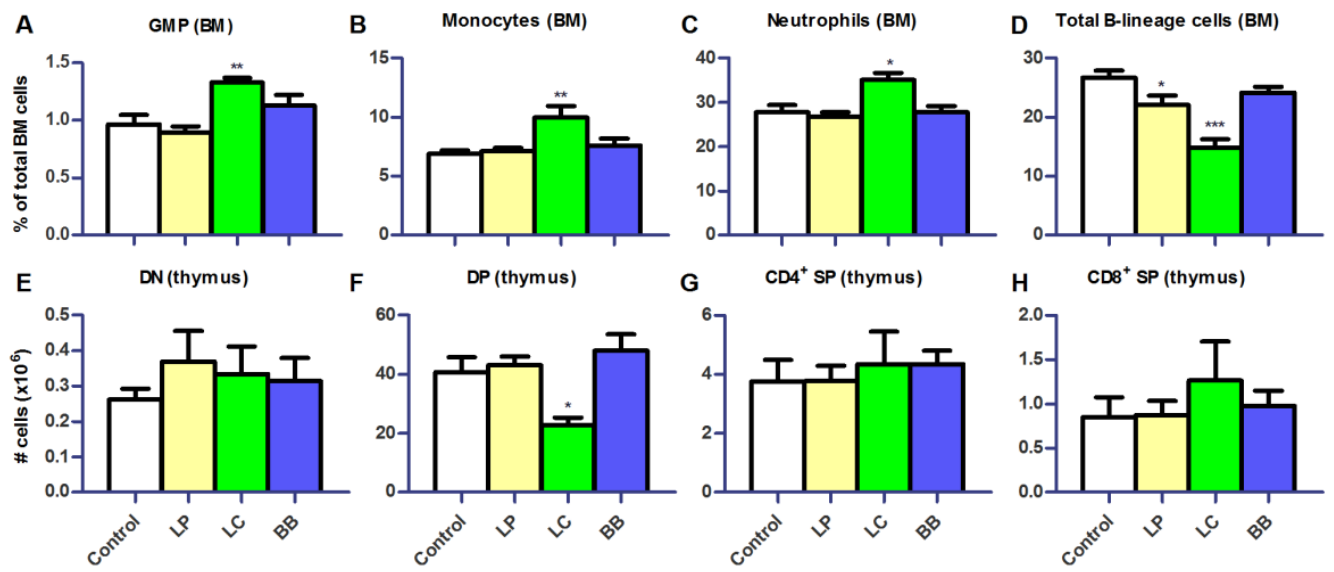

Figure 7. L. casei or L. plantarum supplementation altered myeloid and lymphoid development in bone marrow and thymus of Ercc1 $1^{-1 \Delta 7}$ mice. A-D) Mean frequencies in bone marrow (BM) were determined by flow cytometry. Granulocyte-monocyte precursors (GMP) were defined as Lin CD117 ${ }^{\text {hi }} \mathrm{CD} 11 \mathrm{c}$ CD135 CD16/32+, neutrophils as CD11 b ${ }^{+} L y 6 G^{+}$, monocytes as Ly6C ${ }^{\text {hi }} C D 31^{-}$, and B-lineage cells as $\mathrm{CD}_{19}{ }^{+} \mathrm{CD} 45 \mathrm{R}^{+}$. E-H) Mean absolute numbers were determined by cell counts and flow cytometry. Double negative (DN) cells were defined as $\mathrm{CD}^{-} \mathrm{CD} 4^{-} \mathrm{CD} 8^{-}$, double positive (DP) cells as $\mathrm{CD} 3^{-} \mathrm{CD} 4^{+} \mathrm{CD} 8^{+}, \mathrm{CD} 4^{+}$ single positive (SP) as $\mathrm{CD}^{+} \mathrm{CD} 4^{+} \mathrm{CD} 8^{-}$, and $\mathrm{CD} 8^{+} \mathrm{SP}$ as $\mathrm{CD} 3^{+} \mathrm{CD} 4^{-} \mathrm{CD} 8^{+}$. Data represent the mean + S.E.M. from 4-6 animals per group. ${ }^{*}=p<0.05 ;{ }^{* *}=p<0.01{ }^{* \star *}=p<0.001$. LP $=$ L. plantarum WCFS1; $L C=L$. casei $\mathrm{BL23}$; $\mathrm{BB}=$ B. breve DSM20213.

L. casei supplementation significantly reduced $\operatorname{clgM}^{+} \mathrm{CD} 2^{+}$small resting pre-B slgk $/ \lambda^{+} \operatorname{clg} M^{+} \lg D^{10}$ immature, and $\operatorname{slg} K / \lambda^{+} \operatorname{clg} M^{+} \lg D^{\text {hi }}$ recirculating mature $B$ cells, but did not significantly reduce clgM-CD2 pro- $B$ and clgM $^{+} C D 2^{-}$large cycling pre-B cells (Supplementary Figure 4). In thymus, only L. casei supplementation caused changes 

in cell distribution, with significantly reduced $\mathrm{CD} 3^{-} \mathrm{CD} 4^{+} \mathrm{CD} 8^{+}$double positive (DP) cell numbers (Figure 7E-7H).

No significant changes in GMP, monocytes, neutrophils, and thymocytes were noted in $\mathrm{ErCC1}^{+/+}$mice after bacterial supplementations (Supplementary Figure 5). A significant decrease in B-lineage cells was also observed in $\mathrm{Ercc1}^{+/+}$mice supplemented with L. casei (Supplementary Figure 5D), which predominantly was explained by a decrease in small resting pre-B cells $(p<0.05$; Supplementary Figure $5 E$ ). A tendency to decreased small resting pre-B cells was also observed in $E r c c 1^{+/+}$mice supplemented with L. plantarum $(\mathrm{p}=0.08)$.

\section{L. casei supplementation increases IgG serum titers}

Because $L$. casei supplementation lead to decreased B cell levels in several immune organs of $E r c c 1^{-/ \Delta 7}$ mice, we tested whether serum antibody titers in $E r c c 1^{-/ \Delta 7}$ mice were altered. IgG1 and $\lg G 2 b$ (but not $\lg G 2 a$, $\lg G 3$, $\lg E$, and $\lg A$ ) titers were significantly increased after L. casei supplementation (Figure 8). L. plantarum and B. breve supplementation did not significantly change titers of any $\lg$ subclass.

\section{Serum Ig titers}

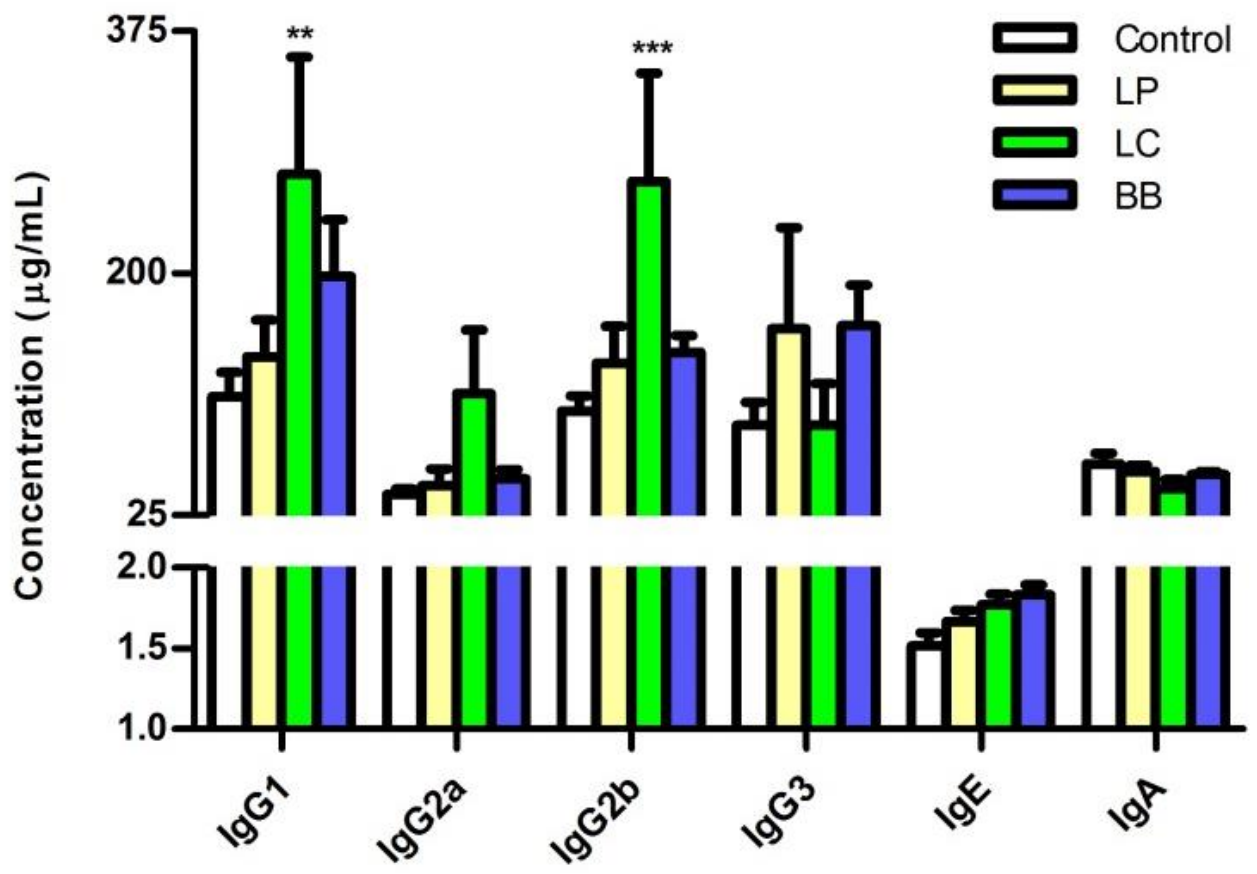

Figure 8. L. casei supplementation increased IgG1 and IgG2b titers in Ercc1 ${ }^{-/ \Delta 7}$ mice. Mean titers of $\lg$ 1, IgG2a, IgG2b, IgG3, IgE, and IgA in serum, as determined by Luminex. Data represent the mean + S.E.M. from 3-6 animals per group. ${ }^{* *}=p<0.01^{* * *}=p<0.001$. LP $=$ L. plantarum WCFS1; $L C=L$. casei BL23; $\mathrm{BB}=$ B. breve DSM20213. 


\section{Bacterial supplementations do not alter lifespan of $\operatorname{Ercc1}^{-/ \Delta 7}$ mice}

The accelerated aging of $E r c c 1^{-1 \Delta 7}$ mice enables to expedite assessment of potential life-extending properties of the bacterial strains. No significant change in lifespan was observed after treating $E r c c 1^{-1 \Delta 7}$ mice lifelong with $L$. plantarum or L. casei (Supplementary Figure 6).

\section{Immune competence improved by $L$. casei and $L$. plantarum supplementation}

To test whether changes in immune cell distribution also impact immune competence, we analyzed the immune response of $E r c c 1^{-1 \Delta 7}$ mice to TNP-KLH. Specific anti-TNP$\mathrm{KLH}$ titers of the three tested isotype classes (IgM, IgG1, IgG2a) after primary and booster immunization were consistently higher after L. plantarum and L. casei supplementation (Figure 9). In particular, IgG1 titers after booster immunization increased in both L. plantarum- and L. casei-supplemented mice compared with control-treated mice $(p<0.001)$.

\section{Anti-TNP-KLH titers}

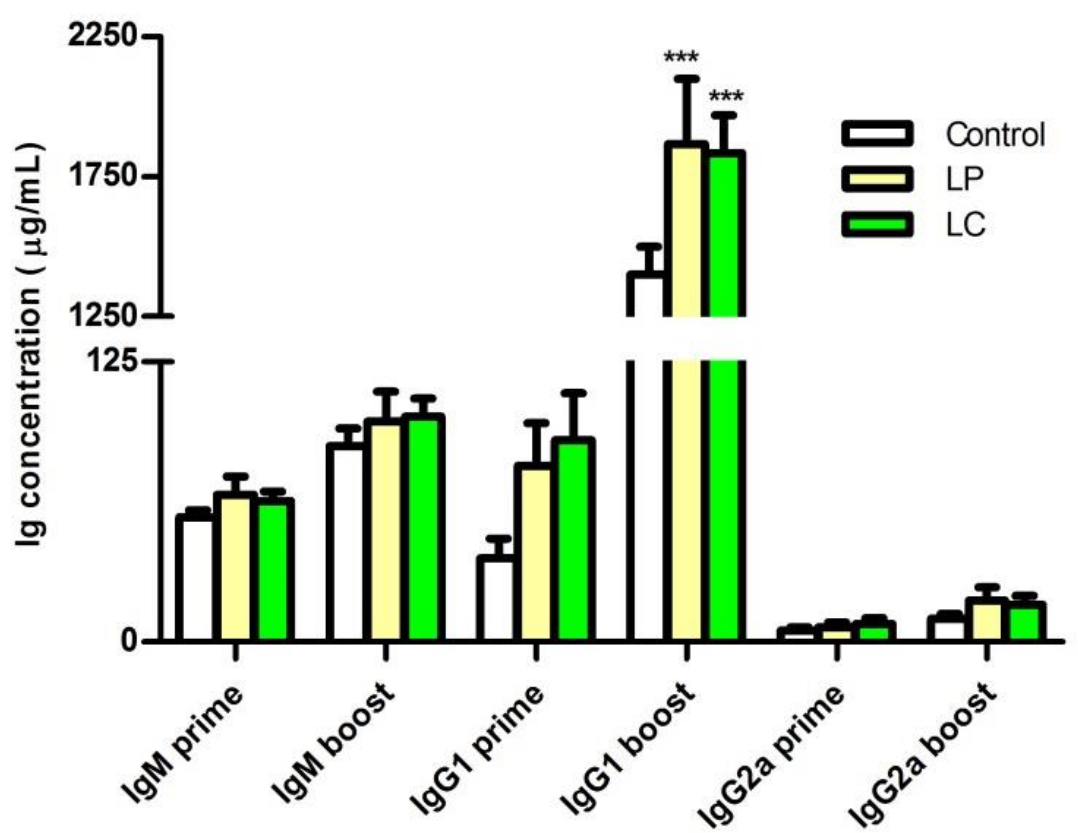

Figure 9. Supplementation of $L$. plantarum and $L$. casei increased specific anti-TNP-KLH antibody responses of $\operatorname{Ercc1}^{-/ \Delta 7}$ mice. Mean TNP-specific $\operatorname{lgM}$, IgG1, and IgG2a concentrations in serum were determined by ELISA, 7 days after primary immunization (prime, age 9 weeks), or 7 days after booster immunization (boost, age 13 weeks). Data represent the mean + S.E.M. of 6-12 animals per group. ${ }^{* * *}=p<0.001$. $\mathrm{LP}=$ L. plantarum WCFS1; LC = L. casei BL23. 


\section{DISCUSSION}

The effects of bacterial supplementations on the intestinal barrier and cellular parameters of immunity were studied in fast aging $\operatorname{Ercc}^{-/ \Delta 7}$ mice. We observed that the mucus layer in the colon declines with age and that bacterial supplementation may prevent or exacerbate the age-related decline in the mucus layer, dependent on the specific bacterial strain. Additionally, we demonstrated a marked difference in the response to bacterial supplementations between $E r c c 1^{-1 \Delta 7}$ mice and WT mice. Finally, supplementation with $L$. casei BL23 profoundly changed the distribution of immune cells and supplementation with L. plantarum WCFS1 or L. casei BL23 improved immune competence in $\mathrm{ErCC}^{-1 / 47}$ mice.

Recently, we showed the age-related decline in mucus barrier of C57BI/ 6 mice as well (Sovran et al, unpublished data). Importantly, we report that the mucus barrier declines with age, in aged $\mathrm{C} 57 \mathrm{BI} / 6$ and $E r c c 1^{-/ \Delta 7}$ mice (Figure 1). This finding adds another age-related phenotype to the wide spectrum of age-related phenotypes observed in $\operatorname{Ercc1}^{-1 / 47}$ mice [58]. Moreover, we report that the age-related decline in mucus barrier can be modulated by bacterial supplementation. L. plantarum prevented the decline in mucus barrier. L. plantarum is able to bind to mucus with a mannosespecific adhesin, which is described as a potential probiotic feature [505]. In total, $L$. plantarum harbors four mucus-binding proteins [506]. Based on the improved spatial compartmentalization of bacteria after $L$. plantarum supplementation, we postulate that $L$. plantarum adheres to the mucus. In addition, we found that $L$. plantarum supplementation tended to decrease the abundance of Akkermansia muciniphila (Figure 3 ), which is known as a mucus degrader [467]. Thus, it would be conceivable that mucus degradation is decreased after $L$. plantarum supplementation. In contrast, $B$. breve is known as a mucus degrader [507], and could therefore be directly responsible for the decrease in mucus thickness in the colon of $B$. breve-treated mice. Interestingly, several pathways involved in protein folding and the UPR were upregulated after $L$. casei and B. breve supplementation (Supplementary Table 2). A high demand for synthesis of secretory proteins (like mucins) induces endoplasmatic reticulum (ER) stress, which in turn induces the UPR [508]. The close proximity of bacteria to the epithelium in $L$. casei- and B. breve-treated mice might induce a high demand for mucin production and secretion, leading to induction of ER stress and UPR. There is indeed evidence that defects in MUC2 mucin and a subsequent defective mucus layer lead to ER stress and UPR [509].

Microbiota profiling showed that only few microbial species are slightly altered by bacterial supplementation (Figure 2, 3). Therefore, most of the observed effects in the mucus barrier and immune system may be directly linked to the supplementation of each of the bacterial strains. 
We found that the different bacterial strains elicited characteristically different responses in gene regulation in the colon (Figure 4). L. plantarum is known for its moderately pro-inflammatory profile, and relatively high IL-10 induction, when tested in human PBMC cultures [457, 472]. In line with these studies, several upstream regulators predicted to be activated after $L$. plantarum supplementation included the inflammatory cytokines IFN- $\gamma$, IL-1 $\beta$, IL-4, and TNF. The association between increased activation of inflammatory cytokines and the improved integrity of the colon after $L$. plantarum supplementation raises the possibility that it might be beneficial to locally increase inflammatory cytokine levels. This suggestion is corroborated by the absence of activation of these inflammatory cytokines after $L$. casei or B. breve supplementation, which did not improve or exacerbate the age-related decline in mucus integrity. A 'tonic' level of constitutive TLR activation by commensal bacteria was previously shown to be crucial in the recovery from DSS-induced epithelial damage due to the role of NF-KB in epithelial repair processes [510]. This notion that "physiological proinflammatory signals" is required for intestinal homeostasis is also supported by studies using epithelium-specific iкB kinase-y (or NEMO) ablation in mice. These mice develop spontaneous colitis due to the failure of NF-KB to induce epithelial repair and steady-state production of innate effector mechanisms in the intestine [511]. TLR2 signaling has been implicated in tight junction regulation in vivo and in vitro [163]. Thus is it possible that aged mice have sub-optimal level of TLR stimulation in the intestine to promote innate barrier defenses and that this is enhanced by L. plantarum, but not by $L$. casei and $B$. breve.

Remarkably, none of the significantly regulated genes were directly linked to mucus production. However, while performing Upstream Regulator Analysis, growth factors like EGF, IGF1, and EGR1 were predicted to be activated after Lactobacillus plantarum supplementation. Together, these findings may indicate that mucus production by goblet cells is not directly enhanced, but is part of general epithelial integrity, supported by a number of growth factors.

Because many regulated genes involved immune-related genes, we additionally analyzed the makeup of the immune system after bacterial supplementation. Whereas supplementation with $B$. breve exacerbated the age-related decline in mucus barrier in colon, it did not cause any changes in mucosal or systemic immunity (Figure 5-8). Oppositely, $L$. casei supplementation caused various signs of inflammation, such as Ly6C $\mathrm{C}^{\text {hi }}$ monocyte and neutrophil influx and production in spleen and BM, respectively. These inflammatory signs were coincided with the general decrease in $B$ cell frequencies (also in the BM) and double-positive thymocytes. There is evidence that neutrophils in the BM are primed by microbial ligands [280]. The effects of microbiotaderived signals on priming $B$ and $T$ cells in the BM have not been previously described. Our study suggests an, up to now, unknown link between microbiota, intesti- 
Supplementation with L. plantarum WCFS1 prevents decline of mucus barrier in colon of $E r c c 1^{-1 \Delta 7}$ mice nal barrier, and B and T cell precursors. Specific precursor stages (i.e. small resting pre-B cells) were significantly decreased after $L$. casei supplementation, and to a lesser extent after L. plantarum supplementation. In the case of L. plantarum supplementation, we suggest that improved intestinal barrier function might alter circulating microbiota-derived products such as peptidoglycan (PGN) and lipopolysaccharide (LPS). For instance, hematopoietic stem cells are damaged after chronic exposure to LPS [512]. Interestingly, the decrease in small resting pre-B cells after L. casei supplementation (and to lesser extent by L. plantarum) was the only finding that could be reproduced in WT mice supplemented with these bacterial strains (Supplementary Figure 4). This may indicate that the effect of $L$. casei and L. plantarum supplementation on $B$ cell development is independent of age.

A previous study showed lifespan extension after B. animalis supplementation [165]. Therefore, we performed a lifespan study for L. plantarum and L. casei, which indicated that neither of them is shortening or extending lifespan (Supplementary Figure 5). Surprisingly, anti-TNP-KLH IgG1 titers in serum increased not only after L. plantarum, but also after $L$. casei supplementation (Figure 9). This increase suggests that a demise in B cell development and B cell distribution does not necessarily translate into impaired B cell function. Previously, it has been shown that antigen-specific antibody titers can be enhanced by probiotic supplementation in aged mice [494], but data on B cell development are lacking.

The effects of the candidate probiotic strains were pronounced on the mucus barrier in the colon of $E r c c 1^{-/ \Delta 7}$ mice compared with WT mice. It has been shown in previous studies that strains such as $L$. casei and $B$. breve have beneficial effects on immunological parameters and intestinal barrier function in young mice [442, 444, 446]. In our hands, $L$. casei and $B$. breve had no effect on mucus barrier or systemic immunity in young WT mice (except for the above-discussed finding on B cell development). A severe deteriorating effect, however, was observed on the mucus barrier or systemic immunity in $\operatorname{Ercc1}^{-/ \Delta 7}$ mice. These findings highlight the need for caution in translating beneficial effects of probiotics observed in young animals or humans to the elderly.

Our study has a number of limitations. We observed remarkable changes in the mucus layer, but could not pinpoint a single gene that is directly linked to the mucus layer. Furthermore, we did not include commercially available probiotic bacterial strains, such as Lactobacillus rhamnosus GG, or a non-probiotic bacterial strain. Nevertheless, our study reveals a previously unknown effect of age on the mucus barrier. We also show that it is possible to modulate this age-related decline in the mucus barrier by supplementation of bacterial strains, with coinciding effects on systemic immunity. More research is warranted to elucidate the interplay between bacteria, the aged gut epithelium, and the immune system. 
Chapter 6

Our data provide evidence that a comprehensive analysis of the intestinal barrier and immunity are needed in order to evaluate how bacterial supplementation contributes to the restoration of the age-related decline in intestinal barrier. A positive finding was that probiotic strains such as $L$. plantarum might contribute to maintenance of intestinal integrity by preventing age-related deterioration of the colonic mucus layer.

\section{ACKNOWLEDGEMENTS}

Authors thank Steven Aalvink, Marjolein de Jong-de Bruijn and Jenny Jansen for technical help. 
Supplementation with L. plantarum WCFS1 prevents decline of mucus barrier in colon of $E r c c 1^{-1 \Delta 7}$ mice

\section{SUPPLEMENTAL INFORMATION}

Supplementary Table 1. Used antibodies in flow cytometry.

\begin{tabular}{|c|c|c|c|}
\hline Target & Format & Clone & Company \\
\hline CD2 & $\mathrm{PE}$ & RM2-5 & $\mathrm{BD}$ \\
\hline \multirow[t]{3}{*}{ CD3e } & APC-Efluor780 & $17 \mathrm{~A} 2$ & Ebioscience \\
\hline & FITC* & $145-2 C 11$ & \\
\hline & PerCP-Сy5.5 & $145-2 \mathrm{C} 11$ & $\mathrm{BD}$ \\
\hline \multirow{2}{*}{ CD4 } & APC-H7 & GK1.5 & BD \\
\hline & FITC & H129.19 & $\mathrm{BD}$ \\
\hline \multirow[t]{3}{*}{ CD8a } & FITC & $53-6.7$ & $\mathrm{BD}$ \\
\hline & $\mathrm{PE}$ & & \\
\hline & V450 & & \\
\hline \multirow[t]{2}{*}{ CD11b } & APC-Cy7 & $\mathrm{M} 1 / 70$ & $\mathrm{BD}$ \\
\hline & PE-Cy7 & & Ebioscience \\
\hline \multirow{2}{*}{$\begin{array}{l}\text { CD11c } \\
\text { CD16/32 }\end{array}$} & PE-Cy7 & N418 & Ebioscience \\
\hline & $\begin{array}{l}\text { APC-Cy7 } \\
\text { Purified }\end{array}$ & $2.4 \mathrm{G} 2$ & $\mathrm{BD}$ \\
\hline \multirow[t]{2}{*}{ CD19 } & $\begin{array}{l}\text { APC-Efluor780 } \\
\text { FITC** }^{*}\end{array}$ & 1D3 & Ebioscience \\
\hline & PerCP-Cy5.5 & & \\
\hline \multirow[t]{2}{*}{ CD25 } & APC & $3 \mathrm{C} 7$ & $\mathrm{BD}$ \\
\hline & PE-Cy7 & PC61.5 & Ebioscience \\
\hline \multirow{3}{*}{$\begin{array}{l}\text { CD31 } \\
\text { CD45R/B220 }\end{array}$} & APC & 390 & Ebioscience \\
\hline & BV421 & RA3-6B2 & $\mathrm{BD}$ \\
\hline & FITC $^{*}$ & & \\
\hline \multirow{7}{*}{$\begin{array}{l}\text { CD68 } \\
\text { CD117 } \\
\text { CD135 } \\
\text { FcERla } \\
\text { FoxP3 } \\
\text { IgD }\end{array}$} & FITC & FA-11 & BioLegend \\
\hline & BV421 & 2B8 & BioLegend \\
\hline & APC & A2F10.1 & $\mathrm{BD}$ \\
\hline & FITC $^{*}$ & MAR-1 & Ebioscience \\
\hline & PE & FJK-16s & Ebioscience \\
\hline & PE & $11.26 .2 \mathrm{ca}$ & BD \\
\hline & PerCP-Efluor710 & $11-26 c$ & Ebioscience \\
\hline Igk & FITC & 187.1 & $\mathrm{BD}$ \\
\hline \multirow{2}{*}{$\begin{array}{l}\lg \lambda \\
\lg M\end{array}$} & FITC & R26-46 & $\mathrm{BD}$ \\
\hline & $\begin{array}{l}\text { APC } \\
\text { Efluor450 }\end{array}$ & $\mathrm{II} / 41$ & Ebioscience \\
\hline \multirow{3}{*}{$\begin{array}{l}\text { Ki-67 } \\
\text { Ly6C }\end{array}$} & PE-Cy 7 & SolA15 & Ebioscience \\
\hline & AF488 & ER-MP20 & AbD Serotec \\
\hline & PerCP-Сy5.5 & HK1.4 & Ebioscience \\
\hline Ly6G & $\begin{array}{l}\text { BV421 } \\
P E\end{array}$ & $1 \mathrm{~A} 8$ & BD \\
\hline Ly6C+Ly6G (GR-1) & FITC* & RB6-8C5 & $\mathrm{BD}$ \\
\hline NK1.1 & FITC* & PK136 & Ebioscience \\
\hline RORyt & AF647 & Q31-378 & $\mathrm{BD}$ \\
\hline TER-119 & FITC* & TER-119 & $\mathrm{BD}$ \\
\hline
\end{tabular}

*Included in lineage cocktail for GMP staining. 
Supplementary Table 2. Top-10 biological processes upregulated (as determined with GSEA) by bacterial supplementations in proximal colon of rcc $^{-1 / 4}$ mice treated with $L$. plantarum WCFS1 (LP), L. casei BL23 (LC), or B. breve DSM20213 (BB). Gene sets significantly regulated ( $<<0.05$, FDR $<0.2$ ) by bacterial supplementations compared with control were determined by gene set enrichment analysis (GSEA). Gene sets involved in immunity are highlighted in orange. Gene sets involved in growth and cell cycle are highlighted in blue.

\begin{tabular}{lllll}
$\mathrm{Nr}$ & Pathway & NES & p-value & FDR q-value \\
1 LP & Malaria (KEGG) & 2.15 & 0.00 & 0.03 \\
2 LP & Fanconi pathway (NCl) & 2.06 & 0.00 & 0.05 \\
3 LP & Glycogen metabolism (WIP) & 2.05 & 0.00 & 0.04 \\
4 LP & ATRBRCA pathway (BIOC) & 1.97 & 0.00 & 0.08 \\
\hline LP & Type II Interferon signaling (WIP) & 1.95 & 0.00 & 0.09 \\
6 LP & VIP Pathway (BIOC) & 1.94 & 0.00 & 0.08 \\
7 LP & ARF6 trafficking pathway (NCI) & 1.92 & 0.00 & 0.09 \\
8 LP & Statin pathway (WIP) & 1.91 & 0.00 & 0.09 \\
9 LP & Fanconi anemia pathway (KEGG) & 1.85 & 0.00 & 0.14 \\
10 LP & IL8/CXCR1 pathway (NCI) & 1.83 & 0.00 & 0.15
\end{tabular}

\begin{tabular}{|c|c|c|c|c|}
\hline $1 \mathrm{LC}$ & Mitotic G1-G1 S Phases (REACT) & 1.89 & 0.00 & 0.08 \\
\hline $2 \mathrm{LC}$ & Unfolded protein response (REACT) & 1.90 & 0.00 & 0.10 \\
\hline $3 \mathrm{LC}$ & S Phase (REACT) & 1.83 & 0.00 & 0.12 \\
\hline 4 LC & DNA replication (KEGG) & 1.91 & 0.00 & 0.12 \\
\hline $5 \mathrm{LC}$ & NOD-like receptor signaling pathway (KEGG) & 1.80 & 0.00 & 0.13 \\
\hline $6 \mathrm{LC}$ & Cone pathway (NCl) & 1.81 & 0.00 & 0.13 \\
\hline 7 LC & DNA replication (WIP) & 1.84 & 0.00 & 0.13 \\
\hline 8 LC & Synthesis of DNA (REACT) & 1.78 & 0.00 & 0.14 \\
\hline 9 LC & Glycosphingolipid biosynthesis - lacto (KEGG) & 1.73 & 0.01 & 0.14 \\
\hline $10 \mathrm{LC}$ & G1 S transition (REACT) & 1.78 & 0.00 & 0.14 \\
\hline $1 \mathrm{BB}$ & $\begin{array}{l}\text { Citrate (TCA) cycle (KEGG) } \\
\text { Prefoldin mediated transfer of substrate to CCT TRIC }\end{array}$ & 2.00 & 0.00 & 0.07 \\
\hline $2 \mathrm{BB}$ & (REACT) & 1.88 & 0.00 & 0.10 \\
\hline $3 \mathrm{BB}$ & $\begin{array}{l}\text { Glycolysis gluconeogenesis (KEGG) } \\
\text { Pyruvate metabolism and citric acid (TCA) cycle (RE- }\end{array}$ & 1.89 & 0.00 & 0.11 \\
\hline $4 \mathrm{BB}$ & ACT) & 1.92 & 0.00 & 0.11 \\
\hline $5 \mathrm{BB}$ & Protein folding (REACT) & 1.75 & 0.00 & 0.12 \\
\hline $6 \mathrm{BB}$ & Fructose and mannose metabolism (KEGG) & 1.82 & 0.00 & 0.12 \\
\hline $7 \mathrm{BB}$ & TCA cycle (WIP) & 1.74 & 0.01 & 0.13 \\
\hline $8 \mathrm{BB}$ & Chaperone-mediated protein folding (REACT) & 1.76 & 0.00 & 0.13 \\
\hline $9 \mathrm{BB}$ & Valine, leucine and isoleucine degradation (KEGG) & 1.77 & 0.00 & 0.13 \\
\hline $10 \mathrm{BB}$ & Butanoate metabolism (KEGG) & 1.72 & 0.01 & 0.14 \\
\hline
\end{tabular}

$\mathrm{NES}=$ normalized enrichment score 
Supplementation with L. plantarum WCFS1 prevents decline of mucus barrier in colon of $E r c c 1^{-1 \Delta 7}$ mice

Supplementary Table 3. Top-10 biological processes downregulated (as determined with GSEA) by bacterial supplementations in proximal colon of rcc $^{-1 / 47}$ mice treated with $L$. plantarum WCFS1 (LP), L. casei BL23 (LC), or B. breve DSM20213 (BB). Gene sets significantly regulated $(p<0.05$, FDR $<$.2) by bacterial supplementations compared with control were determined by gene set enrichment analysis (GSEA). Gene sets involved in immunity are highlighted in orange. Gene sets involved in growth and cell cycle are highlighted in blue.

\begin{tabular}{|c|c|c|c|c|c|c|}
\hline Nr. & Name & Size & ES & NES & $p$-value & FDR q-value \\
\hline $1 \mathrm{LP}$ & GPCRs (WIP) & 163 & -0.47 & -2.12 & 0.00 & 0.00 \\
\hline $2 \mathrm{LP}$ & $\begin{array}{l}\text { Odorant GPCRs (WIP) } \\
\text { Olfactory signaling pathway (RE- }\end{array}$ & 216 & -0.42 & -1.92 & 0.00 & 0.04 \\
\hline 3 LP & ACT) & 44 & -0.53 & -1.89 & 0.00 & 0.04 \\
\hline $1 \mathrm{BB}$ & $\begin{array}{l}\text { IL2 STAT5 pathway (NCI) } \\
\text { Immunoregulatory interactions be- } \\
\text { tween lymphoid/non-lymphoid cells } \\
\text { (REACT) }\end{array}$ & 30 & -0.58 & -2.03 & 0.00 & 0.05 \\
\hline $3 \mathrm{BB}$ & Angiopoietin receptor pathway (NCl) & 47 & -0.50 & -1.91 & 0.00 & 0.05 \\
\hline $4 \mathrm{BB}$ & $\begin{array}{l}\text { Activation of the pre-replicative } \\
\text { complex (REACT) }\end{array}$ & 21 & -0.61 & -1.91 & 0.00 & 0.05 \\
\hline $5 \mathrm{BB}$ & BARD1 pathway (NCI) & 27 & -0.56 & -1.89 & 0.00 & 0.05 \\
\hline $6 \mathrm{BB}$ & Type II Interferon signaling (WIP) & 30 & -0.56 & -1.97 & 0.00 & 0.05 \\
\hline 7 BB & $\begin{array}{l}\text { GPVI-mediated activation cascade } \\
\text { (REACT) } \\
\text { Generation of second messenger }\end{array}$ & 19 & -0.62 & -1.94 & 0.00 & 0.06 \\
\hline $8 \mathrm{BB}$ & molecules (REACT) & 16 & -0.64 & -1.87 & 0.00 & 0.06 \\
\hline $9 \mathrm{BB}$ & IL4 2 pathway ( $\mathrm{NCl}$ ) & 57 & -0.48 & -1.97 & 0.00 & 0.07 \\
\hline $10 \mathrm{BB}$ & IL6 7 pathway (NCI) & 45 & -0.47 & -1.81 & 0.00 & 0.07 \\
\hline
\end{tabular}

$\mathrm{NES}=$ normalized enrichment score. 
A

B cells (PP)

T cells (PP)
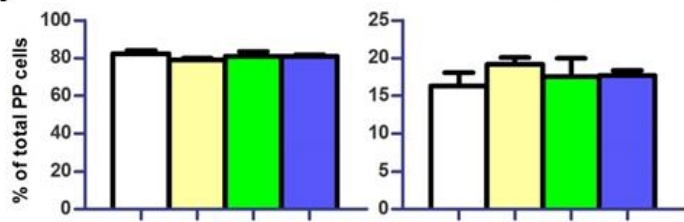

B

B cells (MLN)

T cells (MLN)
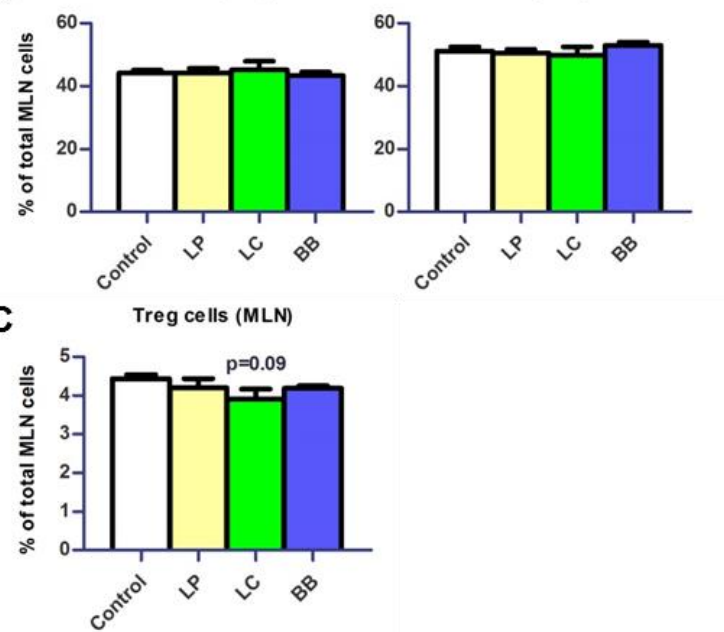

Supplementary Figure 1. Distribution of B cells and T cells in Peyer's patches and mesenteric lymph nodes not changed upon bacterial supplementation in Ercc $^{+1+}$ mice. A/B) Mean frequencies were determined by flow cytometry. B cells were defined as $\mathrm{CD} 19^{+}, \mathrm{T}$ cells were defined as $\mathrm{CD}^{+}$. C) Mean frequencies of $\mathrm{CD}^{+} \mathrm{CD} 4^{+} \mathrm{CD} 8{ }^{-} \mathrm{FoxP} 3^{+}$regulatory $\mathrm{T}$ (Treg) cells in MLN. Data represent the mean + S.E.M. from 4-6 animals per group. $\mathrm{LP}=$ L. plantarum $\mathrm{WCFS1;} \mathrm{LC}=$ L. casei $\mathrm{BL23}$; BB = B. breve DSM20213.

A

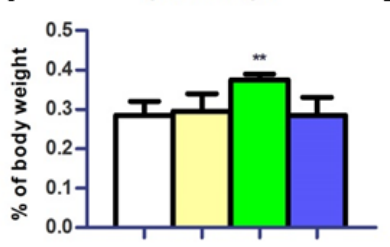

B

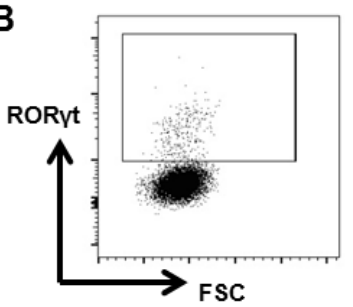

Supplementary Figure 2. Increased relative spleen weight after $L$. casei supplementation of Ercc1 ${ }^{-1 \Delta 7}$ mice. A) Spleen weights relative to body weight. Data represent mean spleen weights + S.E.M of 4-6 animals per group. B) Flow cytometric analysis of splenic Th17 cells. CD ${ }^{+} \mathrm{CD} 4^{+} \mathrm{CD} 8$ cells were gated for RORyt and FSC (forward scatter). 
A

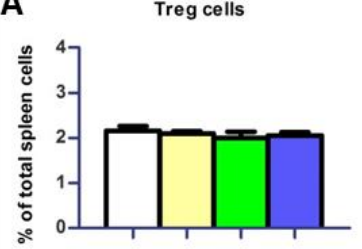

D

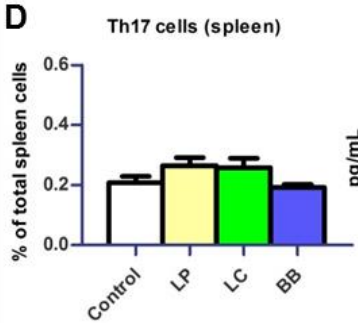

B Ly6C ${ }^{\text {hi }}$ monocytes
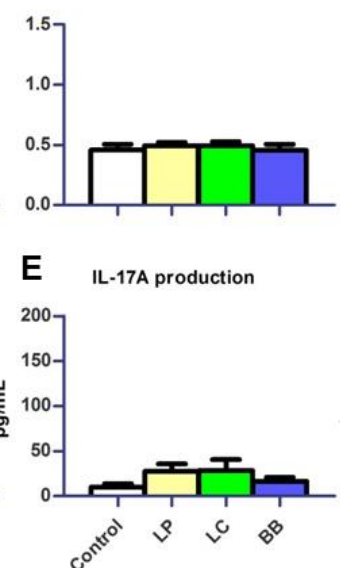

C

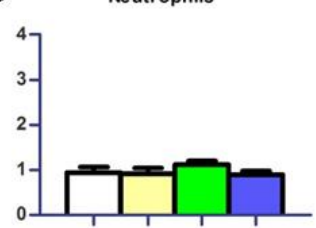

F T cell proliferation

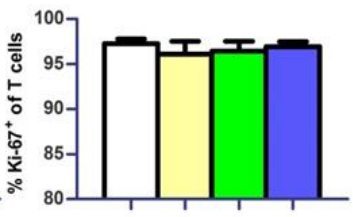

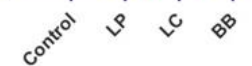

Supplementary Figure 3. Bacterial supplementation of $\mathrm{ErCc}^{+/+}$mice did not change splenic parameters. A) Mean frequencies of Treg cells in spleen. B-D) Mean frequencies of Ly6C ${ }^{\text {hi }}$ monocytes, neutrophils, and $\mathrm{CD}^{+} \mathrm{CD} 4^{+} \mathrm{CD} 8{ }^{-}$Roryt ${ }^{+}$Th17 cells were determined by flow cytometry. E) Mean concentration of IL-17A production by splenocytes stimulated with ConA for four days, as determined by Cytometric Bead Array. F) Mean proliferating $\mathrm{T}$ cells $\left(\mathrm{Ki}-67^{+}\right)$in splenocyte culture stimulated with ConA for four days, as determined by flow cytometry. Data represent the mean + S.E.M. from 4-6 animals per group. LP $=$ L. plantarum WCFS1; LC = L. casei BL23; BB = B. breve DSM20213.

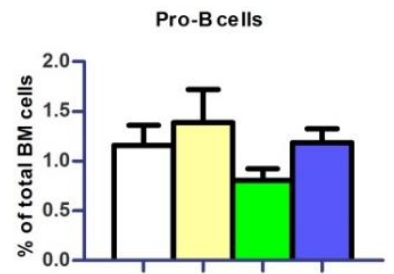

Large cycling pre-B cells

Small resting pre-B cells

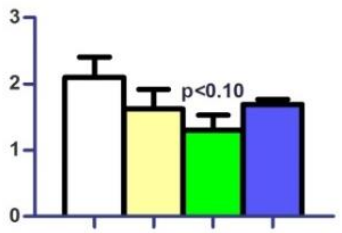

(im)mature Bcells
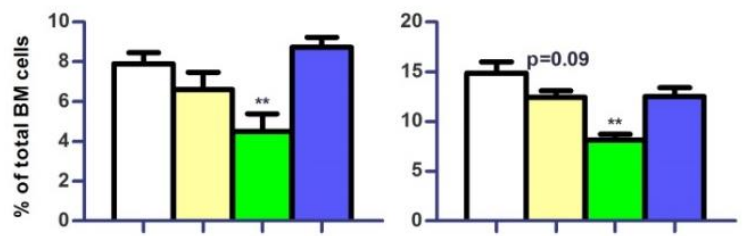

$0^{00^{\circ}}>v^{0}$

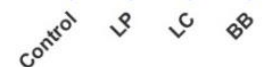

Supplementary Figure 4. L. casei supplementation affected frequencies of subsets of $B$ cell precursors and (im)mature $B$ cells in bone marrow (BM) of $\operatorname{Ercc1}^{-1 \Delta 7}$ mice. Mean frequencies were determined by flow cytometry. B-lineage cells were defined as $C D 19^{+} C D 45 R^{+}$. Mature and immature $B$ cells were defined as slgk $/ \lambda^{+}$, pro-pre-B cells as slgk $/ \lambda^{-}$. Small resting pre-B cells were defined by $\operatorname{clgM}^{+} \mathrm{CD}^{+}$, large cycling pre-B cells by clgM $^{+} \mathrm{CD} 2$, and pro-B cells by clgM ${ }^{-} \mathrm{CD} 2$. Data represent the mean + S.E.M. from 4-6 animals per group. ${ }^{*}=\mathrm{p}<0.05 ;{ }^{*}=\mathrm{p}<0.01$. $\mathrm{LP}=L$. plantarum WCFS1; $\mathrm{LC}=L$. casei $\mathrm{BL23} ; \mathrm{BB}=B$. breve DSM20213. 

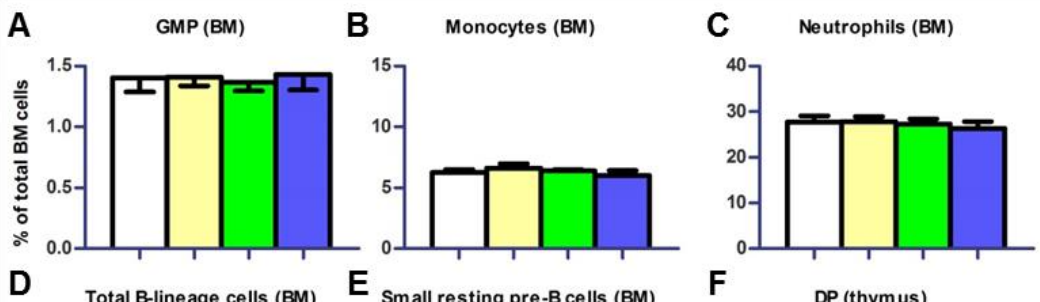

D Total B-lineage cells (BM)

Small resting pre-B cells (BM)

F DP (thymus)
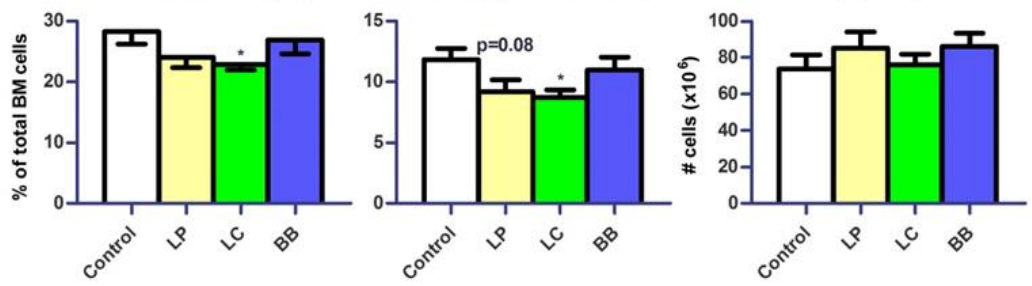

Supplementary Figure 5. L. casei supplementation altered B cell development in bone marrow of $\mathrm{Ercc1}^{+/+}$mice. A-E) Mean frequencies in bone marrow (BM) were determined by flow cytometry. Granulocyte-monocyte precursors (GMP) were defined as $\mathrm{Lin}^{-} \mathrm{CD} 117^{\mathrm{hi}} \mathrm{CD} 11 \mathrm{C}^{-} \mathrm{CD} 135^{\circ} \mathrm{CD} 16 / 32^{+}$, neutrophils as

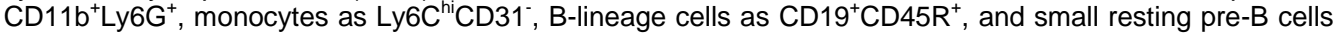
as slgk $/ \lambda^{-} \mathrm{clgM}^{+} \mathrm{CD} 2^{+}$. F) Mean absolute numbers were determined by cell counts and flow cytometry. Double positive (DP) cells were defined as $\mathrm{CD}^{-} \mathrm{CD} 4^{+} \mathrm{CD} 8^{+}$. Data represent the mean + S.E.M. from 4-6 animals per group. ${ }^{*}=p<0.05 . \mathrm{LP}=$ L. plantarum WCFS1; LC = L. casei BL23; BB = B. breve DSM20213.

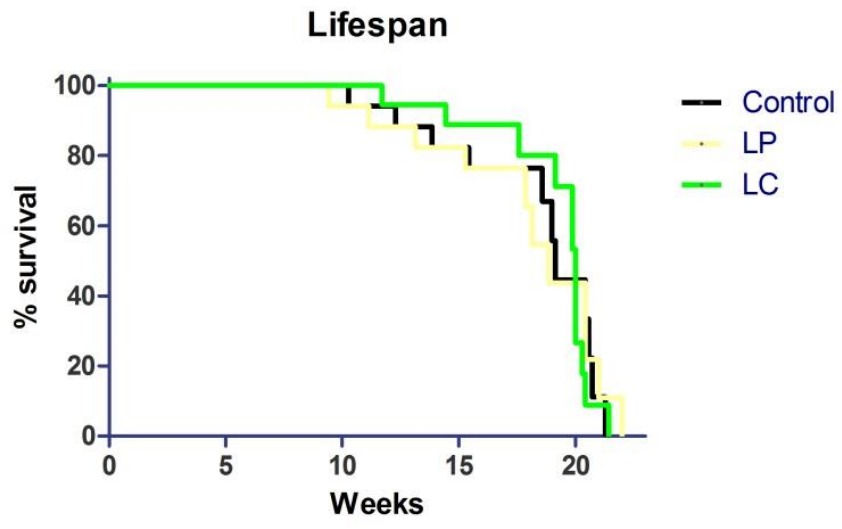

Supplementary Figure 6. Bacterial supplementations did not change lifespan of $E r c c 1^{-1 / 4}$ mice. Data represent $11-12$ animals per group (with an additional 6 animals per group censored at 16 weeks). $L P=L$. plantarum WCFS1; LC = L. casei BL23. 
Supplementation with L. plantarum WCFS1 prevents decline of mucus barrier in colon of $E r c c 1^{-1 \Delta 7}$ mice 


\section{Chapter 7}

\section{Tryptophan restriction arrests B cell development and enhances microbial diversity in WT and prematurely aging $\operatorname{Ercc1}^{-1 / 47}$ mice}

Adriaan A. van Beek ${ }^{1,2,3}$, Floor Hugenholtz ${ }^{1,4}$, Ben Meijer ${ }^{1}$, Bruno Sovran ${ }^{1,5}$, Olaf Perdijk $^{1}$, Wilbert P. Vermeij ${ }^{6}$, Renata M.C. Brandt ${ }^{6}$, Sander Barnhoorn ${ }^{6}$, Jan H.J. Hoeijmakers ${ }^{6}$, Paul de Vos ${ }^{1,7}$, Pieter J.M. Leenen ${ }^{3}$, Rudi W. Hendriks ${ }^{8}$, Huub F.J.

\section{Savelkoul ${ }^{1,2}$}

${ }^{1}$ Top Institute Food and Nutrition, Nieuwe Kanaal 9A, 6709 PA, Wageningen, The Netherlands; ${ }^{2}$ Cell Biology and Immunology Group, Wageningen University, De Elst 1, 6709 PG, Wageningen, The Netherlands; ${ }^{3}$ Department of Immunology, Erasmus University Medical Center, Wytemaweg 80, 3015 CN, Rotterdam, The Netherlands; ${ }^{4}$ Laboratory of Microbiology, Wageningen University, Dreijenplein 10, $6703 \mathrm{HB}$, Wageningen, The Netherlands; ${ }^{5}$ Host-Microbe Interactomics Group, Wageningen University, De Elst 1, 6709 PG, Wageningen, The Netherlands; ${ }^{6}$ Department of Genetics, Erasmus University Medical Center, Wytemaweg 80, 3015 CN, Rotterdam, The Netherlands; ${ }^{7}$ University of Groningen, Pathology and Medical Biology, Hanzeplein 1, $9713 \mathrm{GZ}$, Groningen, The Netherlands; ${ }^{8}$ Department of Pulmonary Medicine, Erasmus University Medical Center, Wytemaweg 80, 3015 CN, Rotterdam, The Netherlands

In press at Journal of Leukocyte Biology 2017, published online at 14 July 2016; doi:10.1189/jlb.1HI0216.062RR 


\section{ABSTRACT}

With aging, tryptophan metabolism is affected. Tryptophan plays a crucial role in induction of immune tolerance and maintenance of gut microbiota. We therefore studied the effect of dietary tryptophan restriction in young wild-type (WT) mice (118-wk lifespan) and DNA repair deficient, premature aged ( $\left.\mathrm{ErCC1}^{-/ \Delta 7}\right)$ mice (20-wk lifespan). First, we found that the impact of aging on distribution of $B$ and $T$ cells in bone marrow (BM) and periphery in 16-week-old Ercc1 $1^{-/ \Delta 7}$ mice is comparable to that in 18-monthold WT aging mice. Dietary tryptophan restriction caused an arrest of $B$ cell development in the BM, accompanied by diminished B cell frequencies in the periphery. In general, old $\operatorname{Ercc1}^{-1 / \Delta 7}$ mice showed similar responses to tryptophan restriction compared with young WT mice, indicative of age-independent effects. Dietary tryptophan restriction increased microbial diversity and made the gut microbiota composition of old $E r c c 1^{-1 / \Delta 7}$ mice more similar to that of young WT mice. The decreased abundances of Alistipes and Akkermansia after dietary tryptophan restriction correlated significantly with decreased $\mathrm{B}$ cell precursor numbers. In conclusion, we report that dietary tryptophan restriction arrests $\mathrm{B}$ cell development and concomitantly changes gut microbiota composition. Our study suggests a beneficial interplay between dietary tryptophan, B cell development, and gut microbial composition on several aspects of age-induced changes. 
Tryptophan restriction arrests B cell development and enhances microbial diversity in WT and $E r c c 1^{-/ \Delta 7}$ mice

\section{INTRODUCTION}

With age, hematopoietic stem cells acquire defects $[65,67]$ and do not efficiently generate lymphoid cells, whereas relatively more myeloid cells are generated [55, 513]. Furthermore, thymus and bone marrow involute, and long-lived B cells accumulate with age, leading to decreased $T$ and $B$ cell production [55, 90, 488]. This immunological decline coincides with gut microbiota composition changes, with e.g. an agerelated decline in Akkermansia abundance [107]. Immunosenescence induces lowgrade inflammation, also known as inflammaging [69-71].

It is known that calorie restriction is associated with lifespan extension [514], but its effects on immunity are not subject of many studies. Tryptophan is involved in the induction of immune tolerance [180] and its breakdown is gradually enhanced during aging [181]. It is also increased in several autoimmune and neurodegenerative diseases [182, 183]. Tryptophan is therefore an important target ingredient to study the impact of nutrient restriction on immunosenescence. Tryptophan is mainly metabolized by indoleamine 2,3-deoxygenase (IDO) leading to production of kynurenine, and is, in addition to being involved in immune tolerance, also essential for maintenance of microbiota diversity $[178,179]$. Decreased serum levels of tryptophan and increased serum levels of kynurenine, suggestive of increased IDO activity, have been observed in elderly people and were associated with elevated inflammatory markers such as IL6 [184]. Dietary tryptophan restriction has been associated with delay of the aging process and longer lifespan in rats [174, 175] and mice [176], but it is unclear what the impact is on immunity and gut microbiota.

The effects of aging can be accelerated by multiple factors [11]. Several mouse models exist that display features of accelerated aging and expedite research on dietary components for healthy aging. Based on a variety of histological, functional, metabolomic, and proteomic data, it has been concluded that the accelerated aging $E r c c 1^{-1 / 4}$ mouse model resembles multiple characteristics of normal murine aging [495]. The excision repair cross-complementation group 1 (ERCC1) protein is involved in at least three repair processes: transcription-coupled repair, global genome nucleotide excision repair and interstrand cross-link repair (and likely sub-pathways of double strand break repair) [515]. Ercc1 ${ }^{-/ \Delta 7}$ mice are deficient for fully functional ERCC1 protein. The mice have a mutated allele, encoding a protein lacking the last 7 amino acids of the protein. Because of the lack of the last amino acids, the interaction between ERCC1 and xeroderma pigmentosum group $F(X P F)$ is less stable and the free proteins are therefore quicker degraded. As a consequence, the expression of ERCC1-XPF DNA repair endonuclease is reduced to about $5 \%$ compared with WT mice [495]. Less ERCC1 protein activity leads to increased accumulation of (primarily endogenous) DNA damage, and consequently enhanced mutation, cellular senescence, and cell death. This results in an accelerated aging phenotype with a lifespan of $\sim 20$ weeks 
(compared with $\sim 118$ weeks in WT mice) $[60,62]$. A recent review pointed out that $E r c c 1^{-/ \Delta 7}$ mice have the broadest spectrum of age-related pathologies, and that they could be useful in the fast screening of interventions to reduce age-related pathology [58].

The aim of this study was to investigate the effects of dietary tryptophan restriction on immunity and gut microbiota in WT mice and in $E r c c 1^{-1 \Delta 7}$ mice as a model for aging. Before testing the effect of dietary tryptophan restriction, the cellular composition of the immune system of $E r c c 1^{-/ \Delta 7}$ mice was evaluated and compared with the aging immune system of WT mice. Because it is well known that aging causes a decline in B cell precursors in bone marrow [516] and T cell precursors in thymus [517, 518], and affects their subsequent distribution in the periphery $[518,519]$, we focused on these cell populations. We found that in particular B cells were affected by long-term tryptophan restriction, and that this effect might be linked with the abundance of specific gut microbes.

\section{MATERIALS AND METHODS}

\section{Mice and genotyping}

Female C57BI/6J mice (3-month-old or 17-month-old) were ordered from Harlan (Horst, NL). Ercc1 $1^{-/ \Delta 7}$ and Ercc1 ${ }^{+/+}$mice (C57BI6/FVB F1 hybrid genetic background) were bred in the animal facility of the Erasmus University Medical Centre (Rotterdam, $\mathrm{NL}$ ). The mice were housed in a specific pathogen-free environment in individually ventilated cages (IVC) in the animal facility of Wageningen University, or in the animal facility of the Erasmus University Medical Centre for the dietary tryptophan restriction experiment. Mice had ad libitum access to AIN93G diet (Research Diet Services, Wijk bij Duurstede, NL), unless otherwise stated. The generation and genotyping of Ercc1 ${ }^{1 \Delta 7}$ mice has been previously described $[60,62]$. All mice were sacrificed at indicated time-points (6 wk, 16 wk, or 18 mo of age). Experiments were conducted with approval of the animal care and use committee of Wageningen University or Erasmus University Medical Centre. Two types of control mice have been used in this study, C57BI/6J mice for the first part of the study, and a mixed genetic background (half C57BI/6J) for the second part of the study. Given the overlap in genetic background, and for reasons of clarity, both C57BI6/J and $\mathrm{ErCC1}^{+/+}$mice are presented as wild-type (WT). Typical unfavorable characteristics, like blindness in an FVB background or deafness in a C57BL6/J background, do not occur in this hybrid background. Mice with a mixed genetic background ( 2 mo or 24 mo of age) were included to compare differences between genetic backgrounds. 
Tryptophan restriction arrests B cell development and enhances microbial diversity in WT and $E r c c 1^{-/ \Delta 7}$ mice

\section{Dietary tryptophan restriction}

Diet with 95\% reduction in tryptophan (hereafter named Trp-restriction) was produced by Research Diet Services with the ingredients of the synthetic AIN93G diet as basis. The total amount of casein (protein source) was reduced from $200 \mathrm{~g} / \mathrm{kg}$ diet to $10 \mathrm{~g} / \mathrm{kg}$ diet. All individual amino acids, except tryptophan, were supplemented back to their original levels, taking into account the purity of each amino acid. Control animals received food similarly reduced in casein but with addition of all amino acids, including tryptophan. Directly after weaning, at 4 wk of age, the animals were divided over the different groups and were given food with or without lower tryptophan levels. Since the mice on Trp-restriction ate approximately $10 \%$ less food compared with control animals, all control animals received $10 \%$ dietary restriction for the whole experimental period.

\section{Organ collection and flow cytometry}

Bone marrow (BM), spleen, mesenteric lymph nodes (MLN), Peyer's patches (PP), and thymus were collected for flow cytometric purposes. PBS lavage of peritoneal cavity was performed to obtain peritoneal cells, which were filtered on a $40 \mu \mathrm{m}$ cell strainer. Single-cell suspensions of bone marrow were obtained by crushing bones with mortar and pestle. Cells were then filtered on a $40 \mu \mathrm{m}$ cell strainer. Spleen, MLN, $\mathrm{PP}$, and thymus single cell suspensions were obtained by gently forcing cells with a syringe on a $40 \mu \mathrm{m}$ cell strainer. Cells were counted using a Z1 Coulter Counter (Beckman Coulter, Fullerton, CA, USA). At least $0.5-2 \times 10^{6}$ cells were stained with Aqua live/dead Efluor506 (Ebioscience, San Diego, CA, USA) stain and for extracellular markers, after which intracellular staining was performed by fixing and permeabilizing cells with Fix/Perm buffer (Ebioscience) according to manufacturer's instructions. Used antibodies are listed in Supplemental Table 1. Stained cells were analyzed on a FACSCanto II (BD Biosciences, Erembodegem, Belgium). FlowJo vX.07 software (Tree Star) was used for data analysis. Doublets were excluded using FSC-A and FSC-H parameters.

\section{Sequencing}

Feces were used for 16S rRNA gene analysis for microbiota profiling. DNA extraction was performed using a combination of the bead-beating-plus column method and the Maxwell 16 Tissue LEV Total RNA purification kit (Promega). Beating of the fecal pellets took place as described before [520], but with STAR (Stool transport and recovery) buffer (Roche). Two hundred and fifty $\mu \mathrm{L}$ supernatant after centrifugation was taken for the Maxwell 16 Tissue LEV Total RNA Purification Kit and the DNA was eluted in $50 \mu \mathrm{L}$ DNAse-free water. Twenty nanogram of DNA was used for the amplification of the 16S rRNA gene with primers 27F-DegS and 338R I + 338R II for $25 \mathrm{cy}$ - 
cles as described before [521], only primers had a UniTag linkers attached; UniTag I (forward) and II (reverses) (I - GAGCCGTAGCCAGTCTGC; II - GCCGTGACCGTGACATCG). The size of the PCR products ( 375 bp) was confirmed by gel electrophoresis using $5 \mu \mathrm{L}$ of the amplification reaction mixture on a $1 \%(\mathrm{w} / \mathrm{v})$ agarose gel containing $1 \times$ SYBR $^{\circledR}$ Safe (Invitrogen). Five $\mu \mathrm{L}$ of these PCR products were taken to add adaptors and an 8-nt sample-specific barcode in an additional 5 cycle PCR amplification. PCR products were purified with magnetic beads (MagBio) according to the HighPrep protocol of the manufacturer's instructions and quantified using the Qubit (Life Technologies). Purified PCR products were mixed in approximately equimolar amounts and concentrated by magnetic beads as the purification before. Purified amplicon pools were 250 bp paired-end sequenced using Illumina Miseq (GATC-Biotech, Konstanz, Germany).

The Illumina Miseq data analysis was carried out with a workflow employing the Quantitative Insights Into Microbial Ecology (QIIME) pipeline [522] and a set of in-house scripts as described before for Illumina Hiseq 16S rRNA gene sequences (RamiroGarcia et al, manuscript in preparation). The set of in-house scripts processed the reads as follows: reads were filtered for not matching barcodes; otu picking and chimera removal was done via matching the sequences to the Silva 111 database, with only one mismatch allowed, and a biom, and with clustalw a multiple alignment and phylogenetic tree file was generated. Further outputs were generated via QIIME, such as filtered reads per sample, PD whole tree diversity measurements and the level 1 to 6 taxonomic distributions with relative abundances.

\section{Statistical analysis}

Values are expressed as mean \pm SEM. Statistical comparisons were performed using the two-tailed Student's $t$ test. We applied the nonparametric Mann-Whitney $U$ test, if no normal distribution was found using the Kolmogorov-Smirnov test. Where no equal variances were observed, we applied the two-tailed Student's $t$ test with Welch's correction. Correlations were determined by Spearman's rank correlation. GraphPad Prism version 5.0.3 (San Diego, CA, USA) was used to perform statistical tests. The microbial composition analysis was performed in Canoco 5.0, where variables were tested for their significance by the Monte Carlo permutation. Differences in microbial genera (L6) were compared using the Wilcoxon test. Values of $p<0.05$ were considered to be statistically significant. Significant differences are indicated by asterisks: ${ }^{*}=$ $p<0.05 ;{ }^{* *}=p<0.01 ;{ }^{* * *}=p<0.001$. 
Tryptophan restriction arrests B cell development and enhances microbial diversity in WT and $E r c c 1^{-/ \Delta 7}$ mice

\section{RESULTS}

$E r c c 1^{-1 \Delta 7}$ mice show an accelerated process of aging, due to deficiency in DNA repair capacity [58]. We compared the aging process of $\operatorname{Ercc}^{-1 / \Delta 7}$ mice (6 wk vs. $16 \mathrm{wk}$ ) with WT controls (16 wk vs. $18 \mathrm{mo}$ ). Information on body weights, organ weights, and organ counts are summarized in Supplemental Table 2. Except for a difference in splenic monocyte numbers with age, no abnormalities were observed in erythroid or myeloid cells (Supplemental Figure 1D).

\section{$B$ and $T$ cell precursors decrease and memory $T$ cells and Tregs increase with aging}

To determine the effects of age on $B$ cells, we first studied the distribution of total $B$ lineage cells $\left(C D 19^{+} C D 45 R^{+}\right)$in BM by flow cytometry. The numbers of $B$-lineage cells tended to decrease with age, but did not reach significance (Figure 1B). B cell precursor frequencies and numbers were reduced, both in aged WT $(p<0.05)$ and 16-weekold $\mathrm{ErCC}^{-1 / \Delta 7}$ mice $(\mathrm{p}<0.001$; Figure $1 \mathrm{~A})$. Immature $B$ cell frequencies were slightly increased in aged WT mice $(p<0.05$; Figure $1 A)$, but were unaltered in numbers (Figure 1B). Mature $B$ cell frequencies were significantly increased in the $B M$ of aged $E r c c 1^{-1 / 4}$ mice, but not in WT mice (Figure 1A). Mature $B$ cell counts in WT were not increased with age, whereas they were increased in aged $\operatorname{Ercc}^{-1 / \Delta 7}$ mice $(p<0.05$; Figure 1B). No major effects were observed in periphery (spleen, MLN, PP), except for a significant age-dependent increase in B cell frequencies in MLN (Supplemental Figure 1D). To validate the effect of the difference in genetic background on $B$ cell development between C57BI/6 and C57BI/6 x FVB F1 mice, we compared young and old F1 mice (2 mo vs $24 \mathrm{mo}$ ), and identified similar changes in $B$ cell development in the $B M$, with mainly a decrease in $B$ cell precursors $(p<0.05$; Supplemental Figure $1 C)$. In the spleen, no significant changes were observed in $\mathrm{B}$ cell numbers between old $\mathrm{F} 1$ mice versus young F1 mice (Supplemental Figure 1F).

Next, we studied the effect of aging on $T$ cell differentiation. Thymus weights decreased with age, accompanied with reduced cellularity in WT mice as well as in $\operatorname{ErcC1}^{-/ \Delta 7}$ mice $\left(\mathrm{p}<0.01\right.$; Supplemental Table 2). Numbers of Lin ${ }^{-} \mathrm{CD}^{-} \mathrm{CD} 4^{-C D} 8^{-}$triplenegative (TN), $\mathrm{Lin}^{-} \mathrm{CD} 3^{-} \mathrm{CD}^{+} \mathrm{CD} 69^{-}$immature single positive (CD8 ISP), $\mathrm{Lin}^{-}$ $\mathrm{CD}^{+} \mathrm{CD}^{+} \mathrm{CD} 8^{-}$single positive (CD4 SP), and $\mathrm{Lin}^{-} \mathrm{CD} 3^{+} \mathrm{CD} 4{ }^{-} \mathrm{CD} 8^{+}$single positive (CD8 SP) cells were (in most cases significantly) reduced in a similar magnitude in normal aged WT and in accelerated aged $E r c c 1^{-1 \Delta 7}$ mice (Figure 1C). 
A

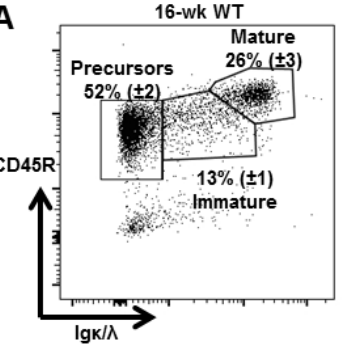

18-mo WT

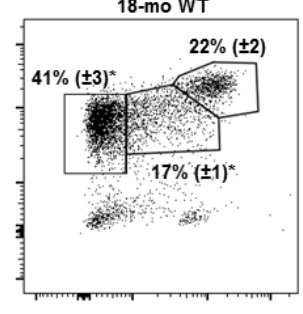

6-wk Ercc1-1/47

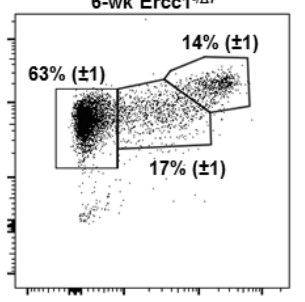

16-wk Ercc1-/ $1 \Delta$

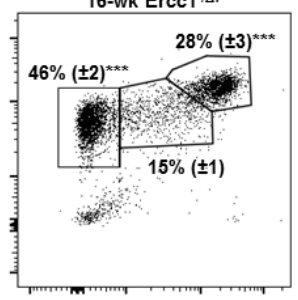

B

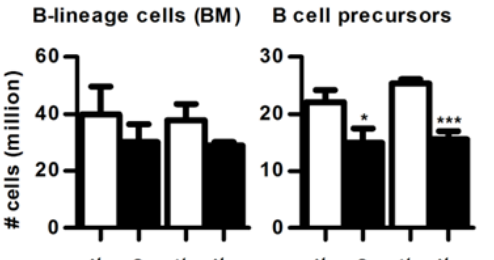

Immature B cells

Mature B cells

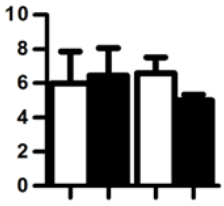

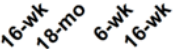

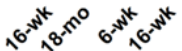

WT Ercc1 ${ }^{-1 \Delta 7}$

WT Ercc1 ${ }^{+\Delta 7}$

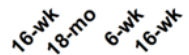

WT Ercc1 ${ }^{\text {tAT }}$

DP

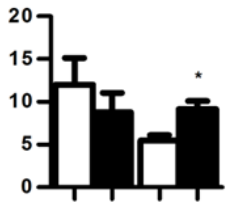

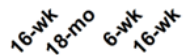

WT Ercc1 ${ }^{\text {tA7 }}$

CD8 ISP
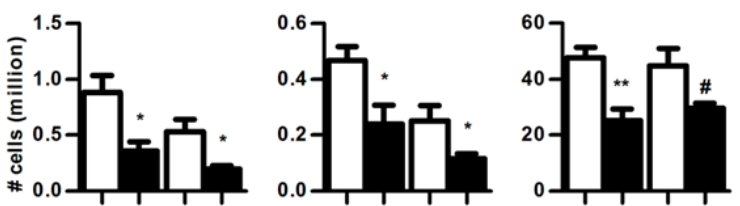

CD4 SP

CD8 SP
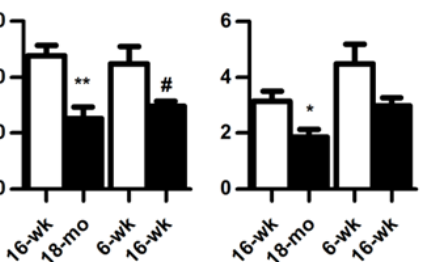

WT Ercc1 $1 \Delta 7$

WT Ercc1 $1 \Delta 7$

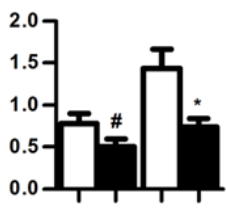

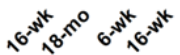

WT Ercc1 $1 \Delta 7$

D

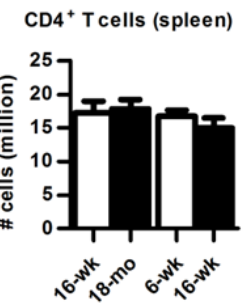

Memory CD4 ${ }^{+} \mathrm{T}$ cells

$\mathrm{CD}^{+} \mathrm{T}$ cells (spleen)

Memory CD8 ${ }^{+}$T cells
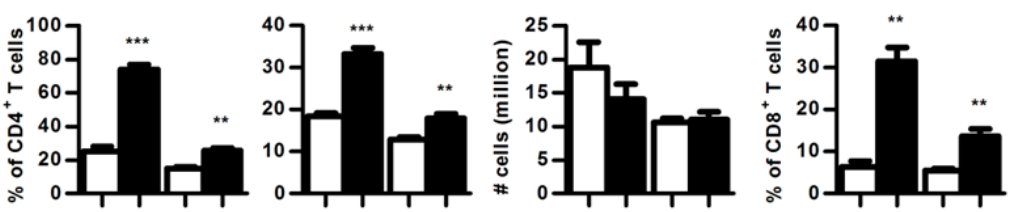

WT Ercc1 $1 \Delta 7$

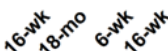

WT Ercc1 ${ }^{+\Delta 7}$

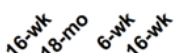

WT Ercc1 $1 \Delta 7$

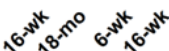

WT Ercc1 $1 \Delta 7$

Figure 1. Aging reduces $B$ and T cell precursors in WT and $E r c c 1^{-1 \Delta 7}$ mice, and increases memory $T$ cells and Treg cells. A) Flow cytometric analysis of $B$-lineage cells in BM. Live single $\mathrm{CD}_{19^{+}}$cells were gated for IgK $/ \lambda$ and CD45R to distinguish IgK/ $/ \lambda^{-} C D 45 R^{+} B$ cell precursors from IgK $/ \lambda^{+} C D 45 R^{+}$immature and $\operatorname{lgk} / \lambda^{+} C D 45 R^{\text {hi }}$ mature $B$ cells. A representative example of each group is shown, with mean frequencies of gated populations within $\mathrm{CD} 19^{+} \mathrm{BM}$ cells \pm SEM. B) Mean absolute numbers of total B-lineage cells, $\mathrm{B}$ cell precursors, immature, and mature B cells were determined by flow cytometry. BM counts based on two femurs, two tibias, two iliac crests, and the sternum. C) Mean counts of triple negative (TN, Lin CD3 ${ }^{-C D} 4$ CD8) cells, CD8 immature single positive (ISP, Lin ${ }^{-}{ }^{-} 3^{-} \mathrm{CD}^{+}{ }^{+} \mathrm{CD} 69^{-}$), double positive (DP, Lin ${ }^{-} \mathrm{CD} 3$ $\left.{ }^{10} \mathrm{CD}^{+} \mathrm{CD}^{+}\right)$, CD4 SP $\left(\mathrm{LinCD}^{+} \mathrm{CD} 4^{+} \mathrm{CD} 8^{-}\right)$, and $\mathrm{CD} 8 \mathrm{SP}\left(\mathrm{Lin}^{-} \mathrm{CD}^{+} \mathrm{CD} 4^{-} \mathrm{CD} 8^{+}\right)$cells with aging. Lineage (Lin) cocktail consisted of CD11b, CD11c, CD19, CD45R, NK1.1, and TER-119. D) Mean counts of CD4 $4^{+}$ cells and $\mathrm{CD} 8^{+} \mathrm{T}$ cells, and mean frequencies of memory $\mathrm{CD} 4^{+} \mathrm{T}$ cells $\left(\mathrm{CD} 44^{\mathrm{hi}} \mathrm{CD} 62 \mathrm{~L}^{-}\right)$, Treg $\left(\mathrm{FoxP}^{+}\right)$, and memory $C D 8^{+} T$ cells $\left(C D 44^{\text {hi }}{ }^{2}\right.$ CD2 $\left.L^{-}\right)$in spleen. Data represent $n=6$ mice, expressed as mean \pm SEM. $\#=p<0.1 ;{ }^{*}=p<0.05 ;{ }^{* *}=p<0.01 ;{ }^{* * *}=p<0.001$. 
Tryptophan restriction arrests $B$ cell development and enhances microbial diversity in WT and $E r c c 1^{-/ \Delta 7}$ mice In spleen, neither $C D 4^{+}$nor $C D 8^{+} T$ cell numbers did change with age in $E r c c 1^{-/ \Delta 7}$ mice (Figure 1D). We found decreased frequencies of $\mathrm{CD} 44^{-/ 10} \mathrm{CD}_{2} \mathrm{~L}^{+}$naïve $\mathrm{CD} 4^{+}(\mathrm{p}<0.05$; data not shown) and increased frequencies of CD62 ${ }^{-}$CD44 $4^{\text {hi }}$ memory CD4 ${ }^{+}(p<0.01)$, memory $\mathrm{CD}^{+} \mathrm{T}$ cells $(\mathrm{p}<0.05)$, and $\mathrm{CD}^{+}{ }^{+} \mathrm{FoxP} 3^{+}$regulatory $\mathrm{T}$ cells $($ Treg; $p<0.01)$ in spleens of aged WT and Ercc1 ${ }^{-/ \Delta 7}$ mice (Figure 1D). Naïve $C D 8^{+} \mathrm{T}$ cell frequencies decreased in aged WT mice only ( $p<0.001$; data not shown).

In MLN, we found decreased $\mathrm{CD}^{+} \mathrm{T}$ cell frequencies with aging $(\mathrm{p}<0.01)$. Alterations in distribution within $\mathrm{CD} 4^{+} \mathrm{T}$ cells were similar as in spleen (Supplemental Figure 1E). Frequencies of total $\mathrm{CD}^{+} \mathrm{T}$ cells decreased and frequencies of memory $\mathrm{CD}^{+} \mathrm{T}$ cells increased in aged WT mice $\left(p<0.001\right.$ and $p<0.01$, respectively), but not in Ercc $1^{-/ \Delta 7}$ mice (Supplemental Figure 1E).

Collectively, these data indicate that the impact of aging on $B$ and $T$ cell precursors in 16-week-old Ercc1 ${ }^{-/ \Delta 7}$ mice is for its major part similar to that of WT aging mice. Furthermore, alterations in distribution of $\mathrm{T}$ cells in periphery are to a large extent similarly present in WT and $\operatorname{Ercc1}^{-/ \Delta 7}$ mice.

\section{Dietary tryptophan restriction arrests B cell development in bone marrow and decreases $B$ cell frequencies in spleen and MLN}

To assess the effect of dietary tryptophan restriction (Trp-restriction) on immune cell development and microbiota, we studied the effect of 12 weeks of Trp-restriction in 16week-old WT mice and Ercc1 ${ }^{-/ \Delta 7}$ mice.

First, we evaluated development of body and spleen weights, and cellularity of spleen, MLN, and BM (Table 1). Average body weight was reduced by up to 2 -fold by Trprestriction $(p<0.001)$. Spleen weight was also reduced by at least 2 -fold $(p<0.01)$. Moreover, relative spleen weight (corrected for body weight) was consistently, albeit not significantly, reduced in WT and Ercc1 ${ }^{-1 \Delta 7}$ mice. Cellularity of spleen was decreased by more than $60 \%$ in WT and $\operatorname{Ercc}^{-1 \Delta 7}$ mice $(\mathrm{p}<0.07)$, while cellularity of MLN was reduced in $E r c c 1^{-1 \Delta 7}$ mice only $(\mathrm{p}<0.05)$. Trp-restriction did not affect BM cellularity in either WT or Ercc1 ${ }^{-/ \Delta 7}$ mice.

Next, we assessed development of B cells in the BM upon Trp-restriction. We observed a reduction in frequencies of $B$-lineage cells within total BM cells $(p<0.03$; data not shown) and numbers of total B-lineage cells in Trp-restricted WT and Ercc1/ ${ }^{-\Delta 7}$ mice ( $p<0.09$; Figure $2 B$ ). We found a near-complete absence of pro-B cells and pre-B cells in the BM of Trp-restricted WT and a complete absence of these cells in the BM of Trp-restricted $E_{r c c} 1^{-/ \Delta 7}$ mice (Figure 1A, B). Immature B cell numbers were decreased by more than 2 -fold in WT and $\operatorname{Ercc1}^{-/ \Delta 7}$ mice $(p<0.1)$, whereas mature $B$ cell numbers were unchanged (Figure $2 \mathrm{~A}, \mathrm{~B}$ ).

To evaluate the consequence of the arrest in $B$ cell development by Trp-restriction, we investigated the distribution of B cells in spleen and MLN. Frequencies and absolute 
numbers of $B$ cells in the spleens of WT and $E r c c 1^{-/ \Delta 7}$ mice were decreased upon Trprestriction (although this did not reach significance; Figure 2D). Follicular (FO) B cells were more affected than marginal zone (MZ) B cells (Figure 2C, 2D). In MLN, frequencies of $B$ cells were significantly decreased by Trp-restriction in WT and Ercc $1^{-/ \Delta 7}$ mice (Figure 2E).

Taken together, these data show that dietary tryptophan restriction arrests $B$ cell development in $\mathrm{BM}$ of 16 -week-old $\mathrm{WT}$ and $\mathrm{ErCc1}^{-/ \Delta 7}$ mice, and affects the ratio of $\mathrm{MZ}$ and FO B cells in the spleen.

\section{T cells and myeloid cells appear less affected by tryptophan restriction}

Trp-restriction decreased total T cell numbers in spleen of WT $(p<0.05)$ and $E r c c 1^{-/ \Delta 7}$ mice $\left(p=0.05\right.$; data not shown), with similar reduction in $C D 4^{+} T$ cell numbers $(p<0.07)$ and $\mathrm{CD}^{+} \mathrm{T}$ cell numbers $(\mathrm{p}<0.05$; Figure $3 \mathrm{~A})$. Reduction in $\mathrm{T}$ cell numbers was in line with the reduction in spleen cellularity. $T$ cell frequencies, however, increased after Trp-restriction, albeit not significantly (data not shown). Hereby, we observed a relative increase in the frequencies of memory $C D 4^{+} \mathrm{T}$ cells in WT and Ercc1 $1^{-/ \Delta 7}$ mice $(p<0.09$; Figure $3 A)$, and to a lesser extent an increase of memory CD8+ T cells in WT mice only $(p<0.08$; Figure $3 A)$. Splenic Treg frequencies were unchanged by Trprestriction (Figure 3A).

In MLN of Trp-restricted WT mice, total CD4 ${ }^{+} T$ cell frequencies $(p<0.01)$ and CD8 ${ }^{+} T$ cell frequencies $(p<0.05)$ were increased after Trp-restriction (Figure 3B). In Trprestricted $E r c c 1^{-1 \Delta 7}$ mice, the same changes were observed for CD4 ${ }^{+} T$ cells $(p<0.05)$ and $\mathrm{CD}^{+} \mathrm{T}$ cells $(\mathrm{p}<0.01)$. However, memory $\mathrm{CD} 4^{+}$and $\mathrm{CD} 8^{+} \mathrm{T}$ cells, and Tregs were significantly increased after Trp-restriction in WT mice only (Figure 3B).

Figure 2 (next page). Dietary tryptophan restriction arrests $B$ cell development in bone marrow (BM) in WT and Ercc1 ${ }^{-\triangle A 7}$ mice, and diminishes $B$ cell numbers in periphery. A) Flow cytometric analysis of $B$ cells in BM. Live single CD19 ${ }^{+}$cells were gated for $\operatorname{lgk} / \lambda$ and $C D 45 R$ to distinguish $\operatorname{lgk} / \lambda{ }^{-} C D 45 R^{+} B$ cell precursors from Igk/ $/ \lambda^{+} C D 45 R^{+}$immature and $\operatorname{lgk} / \lambda^{+} C D 45 R^{\text {hi }}$ mature $B$ cells (upper panels). Continued next page. $B$ cell precursors were further distinguished by $\mu$ and CD2, to identify $C^{-} 2^{-} \mu$ pro-B cells, CD2 ${ }^{-} \mu^{+}$ large cycling pre-B cells, and $\mathrm{CD}^{+} \mu^{+}$small resting pre-B cells (lower panels). A representative example of each group is shown, with mean frequencies of indicated populations within total $B$-lineage cells \pm SEM. B) Mean absolute numbers of $B$ cells, pro- $B$, large cycling pre-B, small resting pre-B, immature, and mature $B$ cells were determined by flow cytometry. C) Flow cytometric analysis of B cells in spleen. Live single CD19 ${ }^{+}$ cells were gated for $\mathrm{CD} 21$ and $\mathrm{CD} 23$ to distinguish $\mathrm{CD} 21^{+} \mathrm{CD} 23^{-}$marginal zone (MZ) B cells from CD21 $\mathrm{CD}^{2} 3^{+}$follicular (FO) B cells and CD21 ${ }^{\circ} \mathrm{CD} 23^{-} \mathrm{B}$ cells. Representative example of each group is shown, with mean frequencies of indicated populations within total splenic $B$ cells \pm SEM. D) Mean absolute numbers of splenic B cells, MZ B cells, and follicular B cells. E) Mean frequencies of CD19+ $B$ cells in mesenteric lymph nodes (MLN). Data represent mean \pm SEM of $n=3-4$ mice per group. $\#=p<0.1 ;{ }^{*}=p<0.05 ;{ }^{* *}=p<0.01$; ${ }^{* * *}=p<0.001$. TrpR $=$ tryptophan restriction. $B M$ counts based on two femurs. 
Tryptophan restriction arrests B cell development and enhances microbial diversity in WT and $E r c c 1^{-1 \Delta 7}$ mice

A
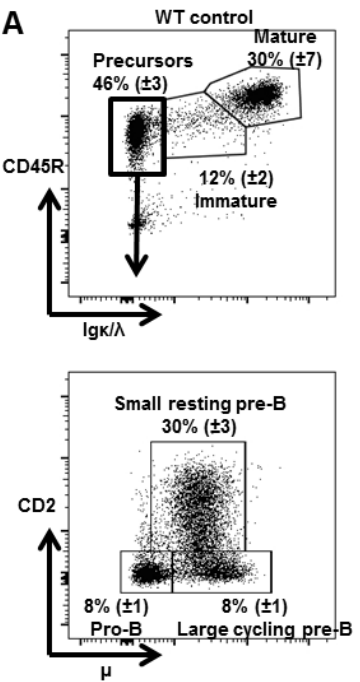
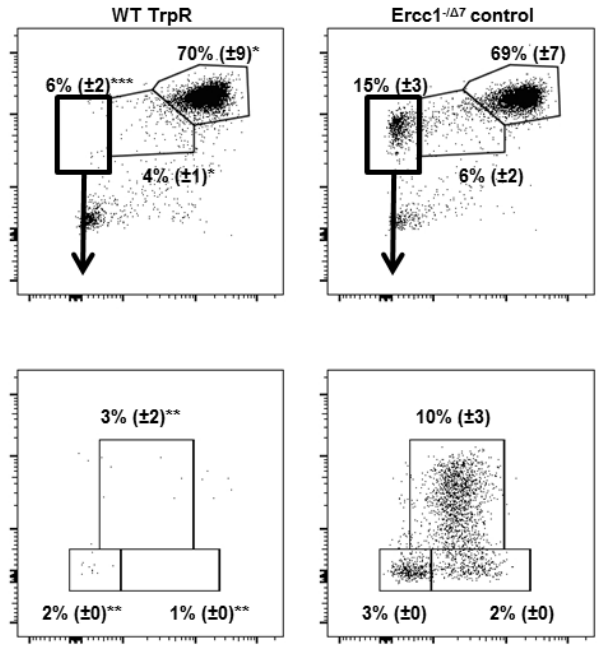

Ercc1 $^{-1 \Delta 7} \operatorname{TrpR}^{-1}$
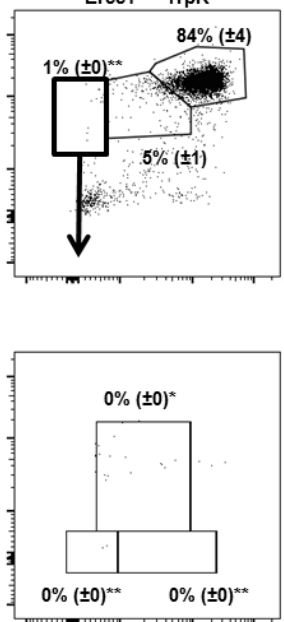

B

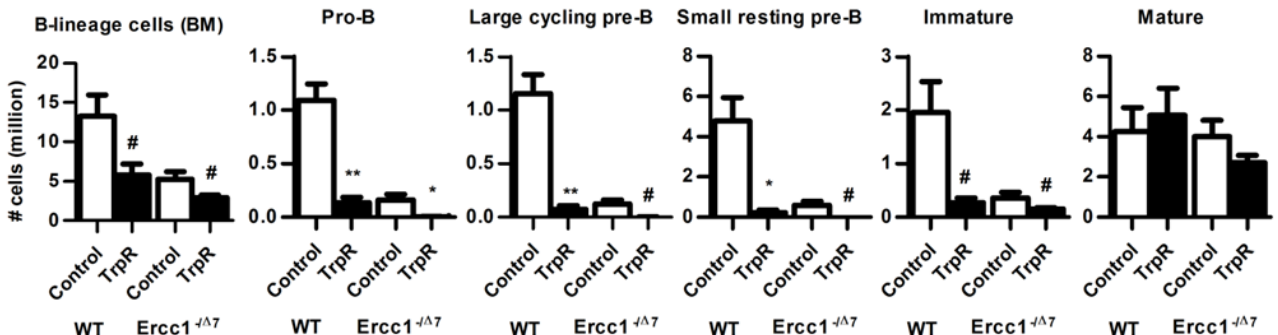

C

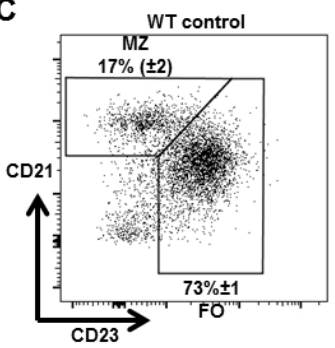

D

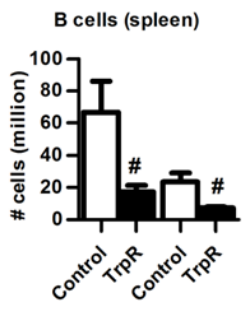

WT Ercc1 $1 \Delta 7$
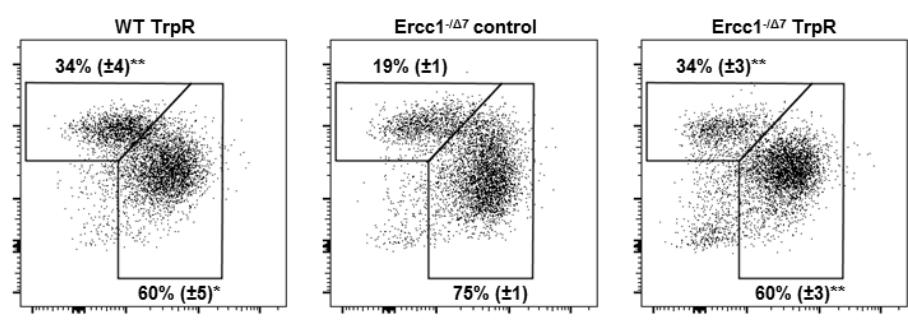
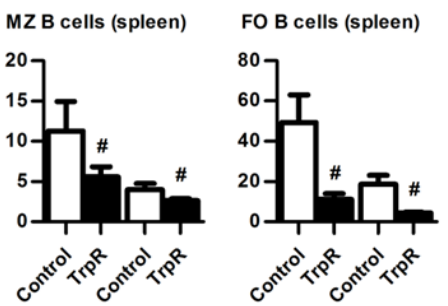

WT Ercc1 $1 \Delta 7$

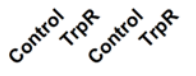

WT Ercc1 147
E

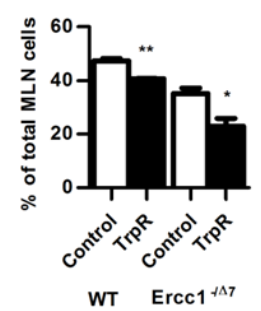




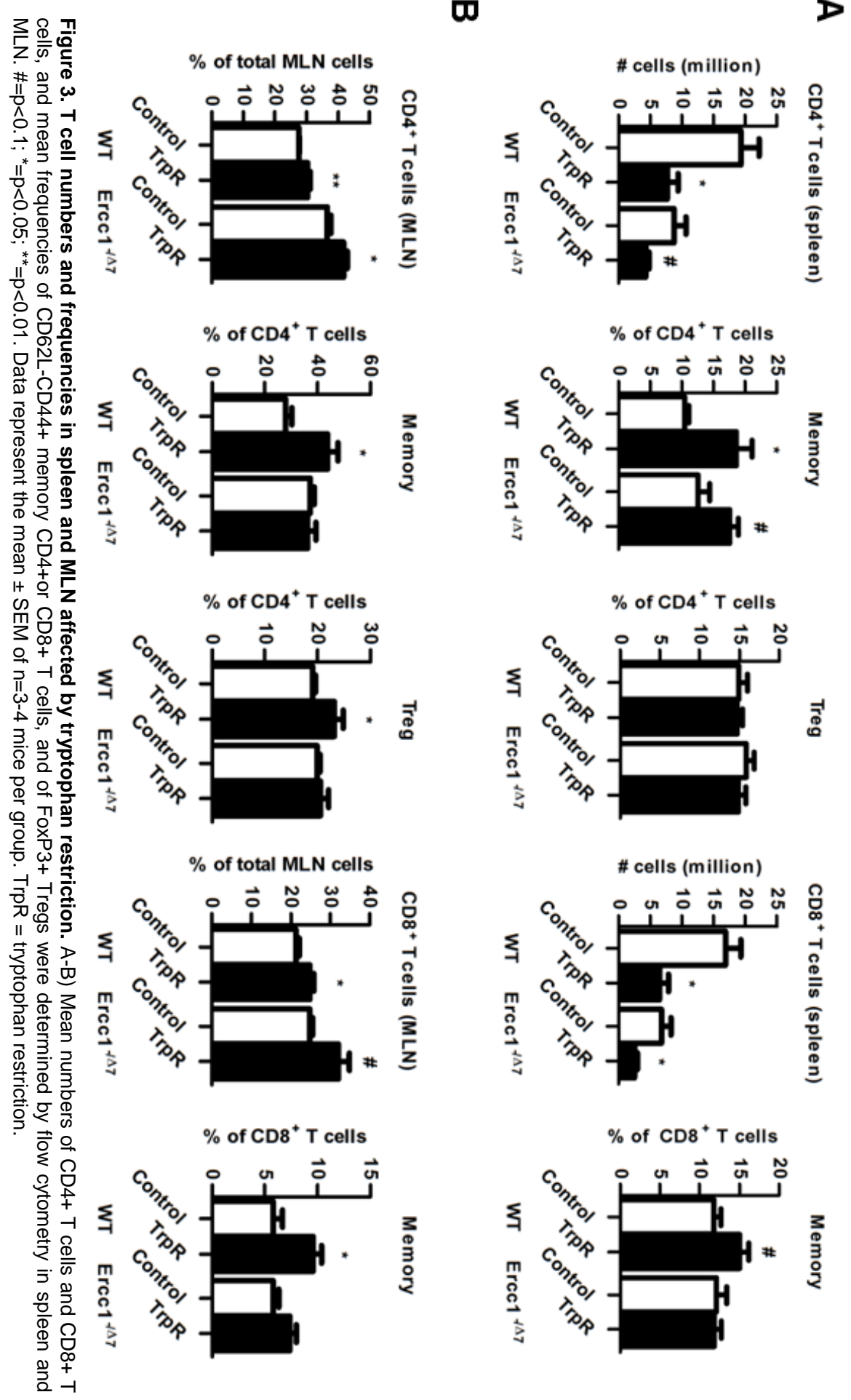


Tryptophan restriction arrests $B$ cell development and enhances microbial diversity in WT and $E r c c 1^{-1 \Delta 7}$ mice Neutrophil numbers in BM were unchanged after Trp-restriction of WT and Ercc1/ $1^{-\Delta 7}$ mice, while monocyte numbers were significantly increased after Trp-restriction of WT mice only ( $p<0.05$; Supplemental Figure 2). Splenic neutrophil and monocyte numbers decreased significantly after Trp-restriction (Supplemental Figure 2).

These observations show that dietary tryptophan restriction affects $T$ cells in the periphery, but to a lesser extent than observed for B cells, and mostly in line with the general decrease of spleen cellularity. In addition, myeloid cell development in BM was unaffected.

\section{Microbiota composition is enriched after tryptophan restriction}

Dietary tryptophan is an important substrate for microbiota metabolism [523]. To study the impact of dietary tryptophan restriction on microbiota composition, we analyzed fecal microbiota composition by $16 \mathrm{~S}$ rRNA gene analysis. Between $2.9 \times 10^{4}$ and $3.2 \times 10^{5}$ reads were obtained per sample. Because a difference in reads may bias the number of species (microbial diversity) found, we performed a rarefaction (Figure 4) to determine the number of reads that are needed to reliably calculate the microbial diversity (phylogenetic diversity; PD) [524]. Control (young) 16-week-old WT mice had significantly higher microbial diversity indexes than (aged) $\operatorname{Ercc}^{-/ \Delta 7}$ mice of the same age (Figure 4). Moreover, Trp-restriction resulted in a higher diversity in both genotypes, with Trp-restricted Ercc1 $1^{-/ \Delta 7}$ mice having similar diversity indexes as WT mice on control diet ( $p<0.05$; Figure 4).

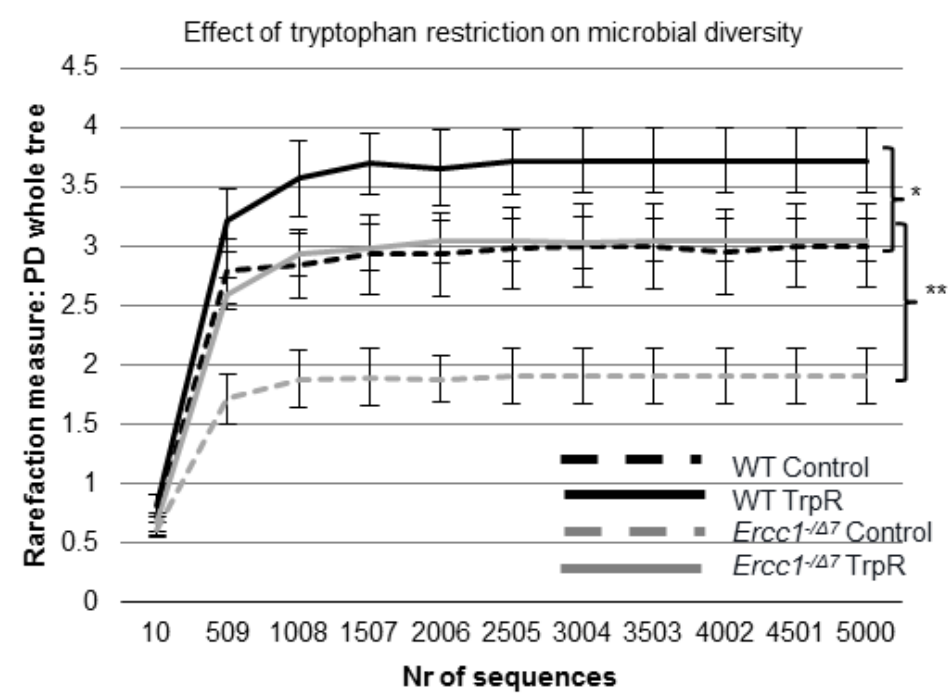

Figure 4. Tryptophan restriction increased microbial diversity in WT and $\operatorname{Ercc1}^{-1 / 47}$ mice. PD whole tree diversity indexes at 5000 sequences are used to calculate differences in diversity. Data represent the mean \pm SEM of $n=4$ mice per group. ${ }^{*}=p<0.05 ;{ }^{* *}=p<0.01$. TrpR $=$ tryptophan restriction. 
To investigate the effect of the diet and the genotypes on the microbial profile, a multivariate analysis was carried out, as previously published [107]. The resulting ordination plots shows the redundancy analysis (RDA). The differences shown in the RDA are significant $(p<0.05)$. On genus level $(L 6)$, the RDA indicated that both genotype and diet had a significant impact on the microbial composition $(p<0.05)$. The RDA plot showed a distinct microbial profile for each of the experimental groups (Figure 5). A total of $34.9 \%$ of the total variation in the microbial dataset could be related to diet and genotype. Most of the variation is plotted on the first ordination axis $(20.0 \%)$, and mainly separates on the genotype, whereas the second ordination axis mainly separates on the diet $(9.7 \%)$. The microbial composition of (aged) $\operatorname{Ercc}^{-1 / 47}$ on Trprestriction closely resembled the microbial composition of (young) Trp-restricted WT mice, implying that Trp-restriction preserved microbial composition in the premature aging repair mutant.

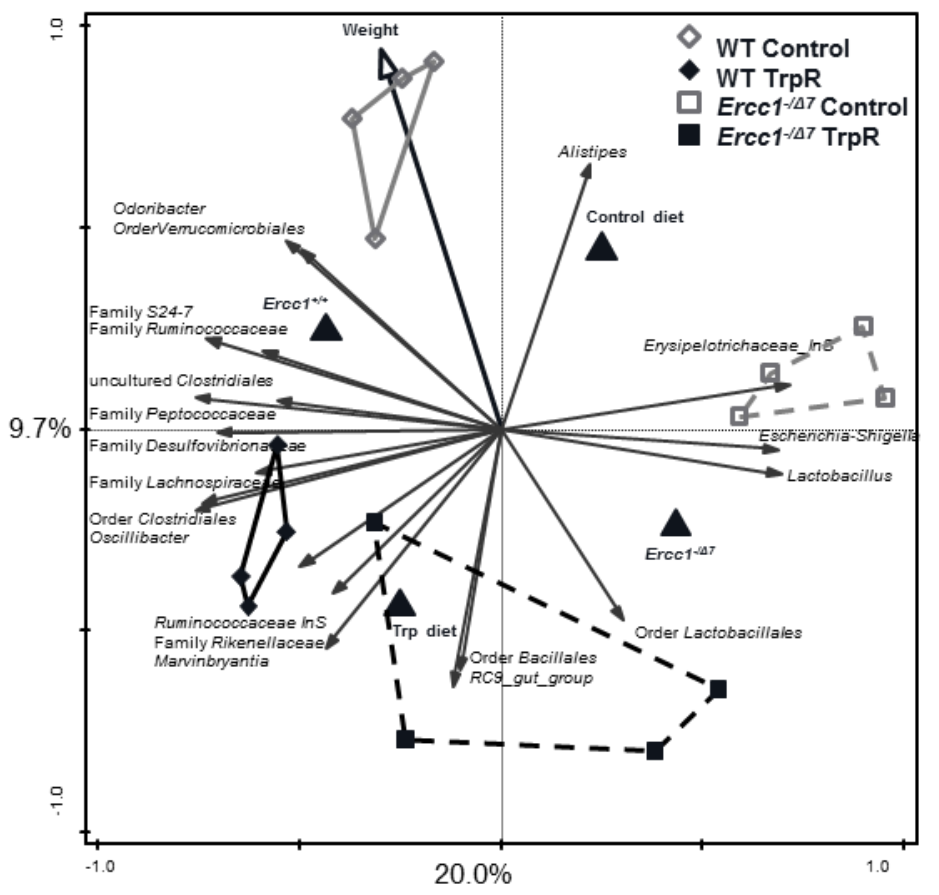

Figure 5. Tryptophan restriction changes microbial composition in the gut of WT and Ercc1 ${ }^{-\Delta /}$ mice. Redundancy analysis on genus level (L6). Mice belonging to WT control, WT TrpR, Ercc1 ${ }^{-1 / 47}$ control, and Ercc $^{-1 / \Delta 7}$ TrpR are indicated by gray diamonds, black diamonds, gray squares, and black squares, respectively. Besides the abundance of microbial species, genotype, diet, and weight were included in the variables, and together they explain $34.9 \%$ of the variability in the dataset. First and second ordination axes are plotted, showing $20.0 \%$ and $9.7 \%$ of the variability in the dataset, respectively. The third ordination axis explains an additional $5.2 \%$ of the variability in the dataset (not shown). Both genotype and diet had a significant impact on the microbial composition $(p<0.05)$. Each small symbol represents one animal. Larger triangle symbols represent the two genotypes or two diets. $\operatorname{TrpR}=$ tryptophan restriction. 

Decreased relative abundances of Parabacteroides, Lachnospiraceae, and Ruminococcaceae were found in control Ercc1 ${ }^{-1 / 47}$ mice compared with control WT mice (Figure 6). Relative abundance of Lactobacillus was increased more than 6-fold, and relative abundance of Akkermansia was decreased more than 20 -fold, albeit not significantly. Tryptophan restriction of WT and $E r c c 1^{-1 / 7}$ mice resulted in significantly higher abundances of the Bacteroidetes RC9 gut group and the order Clostridiales, and lower abundances of Alistipes in the top 15 abundant species as indicated in Figure 6.

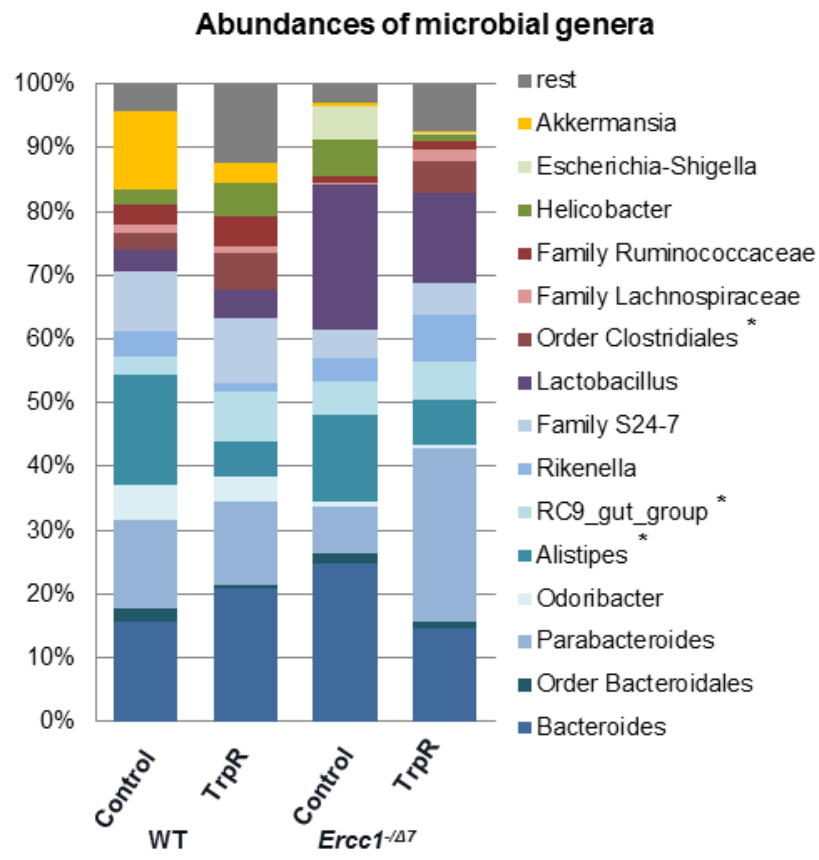

Figure 6. Tryptophan restriction leads to higher abundances of Bacteroidetes RC9 gut and Clostridiales, and lower abundance of Alistipes. Relative abundance profile of the top 15 most abundant genus level groups, where 'rest' indicates all other groups, including the unclassified groups. ${ }^{*}=p<0.05$. Data represent the mean \pm SEM of $n=4$ mice per group. TrpR $=$ tryptophan restriction.

Finally, we correlated the abundance of microbial species in all different mouse samples to the $B$ cell precursor numbers (pro-B cells and pre-B cells together) to investigate a putative association between microbiota composition and the main immune effect of Trp-restriction. We found that Alistipes and Akkermansia positively correlated with $B$ cell precursor numbers $(p<0.05$; Figure 7$)$, with no other microbial species correlating with $\mathrm{B}$ cell precursor numbers. We also tested for correlation of pro-B or pre-B cells with specific microbiota. Akkermansia consistently correlated with pro-B cells $(p<0.01$; Figure 7$)$, large cycling pre-B cells $(p<0.05$; data not shown), and small resting pre-B cells $(p<0.05$; data not shown), while Alistipes only significantly correlated with small resting pre-B cells $(p<0.05$; data not shown). 
Thus, we found a profound impact of dietary tryptophan restriction on fecal microbial composition, and that Alistipes and Akkermansia abundances were positively correlated with $B$ cell precursor numbers.
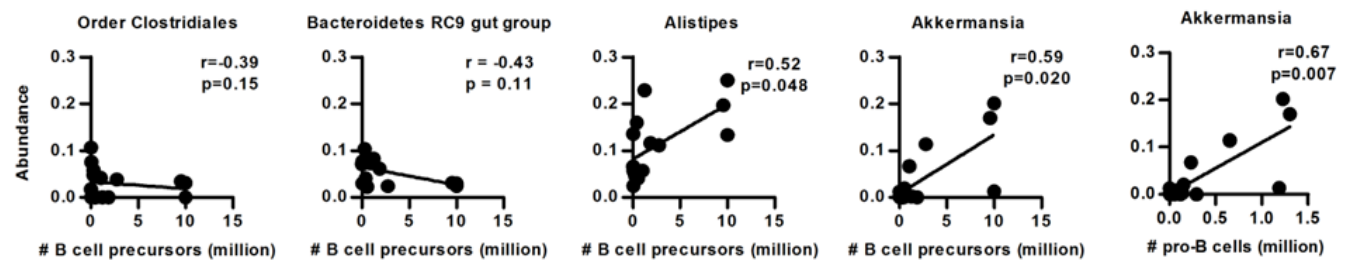

Figure 7. Correlation between $B$ cell precursor numbers and abundance of Alistipes and Akkermansia. Spearman correlation between total B cell precursor numbers (pro-B cells plus pre-B cells) or pro-B cell numbers and abundance of Alistipes and Akkermansia. Each circle represents an animal (control and tryptophan-restricted mice together used in correlation analysis).

\section{DISCUSSION}

Four key findings have been made in this study, namely (1) the prematurely aging $\operatorname{Ercc1}^{-/ \Delta 7}$ mice largely resemble normal mice in age-related changes of the immune system; (2) dietary tryptophan restriction similarly arrests B cell development in bone marrow of 16-week-old WT and Ercc1 ${ }^{-1 / \Delta 7}$ mice, and affects B cells much more than $T$ cells or myeloid cells; (3) dietary tryptophan restriction increases gut microbial diversity in 16-week-old WT and Ercc1 ${ }^{-/ \Delta 7}$ mice; and (4) the abundance of Alistipes and Akkermansia is positively correlated with the number of $\mathrm{B}$ cell precursors.

In this study, we compared the aging process of normal aging WT mice (16-week-old vs. 18-month-old) with the accelerated aging process of $E r c c 1^{-1 / 4}$ mice (6-week-old vs. 16-week-old). When determining cell frequencies (Figure 1, Supplemental Figure 1), most of the changes we observed for the aging process in WT mice were also observed for the $E r c c 1^{-1 \Delta 7}$ model, and were in line with previous findings. This should not be interpreted as a suggestion that the model completely resembles natural aging. The model is used for specific age-induced anomalies that are accelerated in $\mathrm{ErCC}^{-1 / \Delta 7}$ mice. These include a decrease in B cell precursors in BM [55], an increased memory formation [525], and an increased Treg formation [526]. We feel this model allows us to test interventions on these parameters such as food restriction experiments in an expedited way. Nevertheless, others have concluded that the aging process in other organs of $\operatorname{Ercc}^{-/ \Delta 7}$ mice resembles that of normal aging mice based on histological, proteomic, and metabolomic parameters [495].

When comparing the decline in B cell development in 16-week-old Ercc1 ${ }^{-1 / 7}$ mice (Figure 1) with the decline as observed in control-fed 16-week-old Ercc1 ${ }^{-/ \Delta 7}$ mice (Figure 2), a difference in the magnitude of decline in B cell precursors was observed. Two major aspects, however, may explain the difference between the data as presented in 
Tryptophan restriction arrests B cell development and enhances microbial diversity in WT and $E r c c 1^{-/ \Delta 7}$ mice Figure 1 and Figure 2. First, control-fed mice (Figure 2) received 10\% dietary restriction. Second, feed for control-fed mice (Figure 2) was produced in a way to remove tryptophan. Both aspects might have had an unknown influence on the B cell compartment in the BM, which prevent from a direct comparison. Nevertheless, the decline in B cell development as such (as a trend) is confirmed by the data as presented in both figures.

The mechanisms by which dietary tryptophan restriction impacts on immunity and microbiota have not been studied so far. Surprisingly, tryptophan-restricted mice showed a profound decrease in body weight (Table 1). This decrease in body weight could not be accounted for food intake, which was similar to their controls. This finding indicates that dietary tryptophan restriction induces metabolic changes. There is a remarkable similarity between the effect of dietary tryptophan and methionine restriction on body weight. Dietary methionine restriction decreased body weights of mice by more than $30 \%$, and increased resistance of liver cells to oxidative stress [173, 527]. In addition, dietary methionine restriction decreased the generation of oxygen radicals from mitochondria, and subsequent damage to mitochondrial DNA and proteins [528]. Energy expenditure in methionine-restricted rats was 1.5 -fold higher, which was linked to metabolic changes in brown and white adipose tissues [529]. Further research is warranted to understand how dietary tryptophan restriction causes such a marked weight loss. It would be of interest to investigate levels of cytokines and subsequent effects on immunity that might explain our data on development of B cells in the BM but not myeloid cells. Interestingly, adiponectin levels were increased after dietary methionine restriction [529]. Adiponectin is produced by adipocytes, which are also present in the BM. Adiponectin indirectly (via stromal cells) inhibits lymphopoiesis in early B cell precursors, but slightly enhances myelopoiesis in myeloid precursors [530]. Lowered adiponectin levels in the BM could thus explain the arrest in B cell development but not myeloid cell development.

Strikingly, we found a pronounced effect on B cells, which precursors were almost absent from the BM after dietary tryptophan restriction (Figure 2). It suggests that B cell development critically requires a lifelong need for tryptophan or its metabolites. The decline of the downstream tryptophan metabolite nicotinamide adenine dinucleotide $(\mathrm{NAD})^{+}$with aging [531] might explain the decrease in $\mathrm{B}$ cell precursors with aging that was reported [55,532] and we have described here. Interestingly, $N A D^{+}$is produced by nicotinamide phosphoribosyltransferase (NAMPT) [533]. NAMPT also declines with aging [531], and it exerts anti-apoptotic functions [534]. NAMPT was initially identified as pre-B cell enhancing factor, because of its capacity to enhance pre-B cell colony forming [535]. A role for NAMPT in enhancing early B cell stages could explain our finding that $B$ cell development is dependent on age and dietary tryptophan supplies. 
We found that in spleen and MLN, B cell numbers and frequencies were more affected by Trp-restriction (Figure 2) than T cells (Figure 3) or myeloid cells (Supplemental Figure 2). These data demonstrate that B cells are particularly affected by Trp-restriction, in contrast to cells from erythroid and myeloid lineages.

In the comparison of WT and Ercc1 ${ }^{-/ \Delta 7}$ mice, we observed that the $\operatorname{Ercc}^{-1 / \Delta 7}$ genotype caused a decrease in microbial diversity (Figure 5), in line with the prediction that microbial diversity in aging humans decreases [132]. Dietary tryptophan restriction increased microbial diversity and composition (Figure 5-7) and it might therefore prove to be a valuable nutritional intervention to improve age-related decline of gut microbial diversity.

The abundance of two microbial species (Alistipes and Akkermansia) was positively correlated with the decline of B cell precursors (Figure 7). Low abundance of Akkermansia correlated with lower numbers of each of the different $B$ cell precursor stages (pro-B, large cycling pre-B, and small resting pre-B). Akkermansia muciniphila is positively correlated with health status, as its numbers are lower in elderly compared with adults [536], and are lower in obese adults compared with healthy adults [462]. Abundance of Alistipes is decreased in HIV-positive patients [537] and in patients suffering from inflammatory bowel disease [538], which is characterized by overexpression of IDO in the colon [539]. Importantly, Akkermansia and Alistipes are both known to harbor tryptophanase, which transforms tryptophan into indole [540,541], indicating that these microbes might be dependent on a direct source of tryptophan. However, so far no evidence has been reported that abundances of Alistipes and Akkermansia are related to tryptophan metabolism, or whether these two species are extremely sensitive to tryptophan restriction. Interestingly, Zelante et al found that tryptophan metabolites from microbiota engage with the aryl hydrocarbon receptor and balance mucosal reactivity via IL-22 [523], providing the first evidence that tryptophan metabolism by gut microbiota shapes immunity.

Trp-restriction strongly altered the immune system of aged $E r c c 1^{-1 / 7}$ mice, indicating the high responsiveness of the model to dietary interventions. When comparing WT mice with $\operatorname{Ercc}^{-1 \Delta 7}$ mice, we found similar trends for the majority of the measured parameters, showing an age-independent effect of Trp-restriction. However, the effect of Trp-restriction results in a further increase of the effect as seen in aging of $E r c c 1^{-1 \Delta 7}$ mice in B cell precursors and microbiota composition. The combined effect of Trprestriction and aging is reflected by a more pronounced impact on $B$ cell precursors (Figure 2) and microbiota composition (Figure 5). With aging, the loss of lymphoid tissue in the BM leads to a decline in B cell lymphopoiesis in the BM [55, 90]. Recently, a progressive decline in blood $B$ cells was observed in aging [73]. The ratio of naive/memory B cells is reduced with aging [94]. In addition to the decreased generation, functional defects are present in B cells of elderly. Humoral immune responses are 
Tryptophan restriction arrests B cell development and enhances microbial diversity in WT and $E r c c 1^{-/ \Delta 7}$ mice impaired in elderly [96], accompanied by a decrease in total antibody production, but an increase in auto-antibody production [97, 98]. A demise in B cell precursors after Trp-restriction could therefore imply an exacerbated functional impairment to mount a proper humoral immune response. An antigen-specific challenge, like TNP-KLH, could give insight in the ability to mount a humoral immune response.

In conclusion, our results show that dietary tryptophan restriction is a powerful intervention to shape immunity and gut microbiota, also in aging. Dietary tryptophan restriction arrests B cell development. Further studies are warranted to investigate the role of microbial or host tryptophan metabolism and the changing gut microbiota composition on the major effect of dietary tryptophan restriction on B cell development. In particular, more studies are needed to directly test if the microbiota instructs B cell development in the BM.

\section{ACKNOWLEDGEMENTS}

Ineke Heikamp-de Jong is acknowledged for support during experimental work. We would like to thank Sangeeta Badloe and the animal caretakers for general assistance with mouse experiments. 


\section{SUPPLEMENTAL INFORMATION}

Supplemental Table 1. Used antibodies for flow cytometric procedures.

\begin{tabular}{|c|c|c|c|}
\hline Target & Format & Clone & Company \\
\hline CD2 & $\mathrm{PE}$ & RM2-5 & $\mathrm{BD}$ \\
\hline \multirow[t]{4}{*}{ CD3e } & APC-Efluor780 & $17 \mathrm{~A} 2$ & Ebioscience \\
\hline & APC-Cy7 & $145-2 \mathrm{C} 11$ & \\
\hline & $\begin{array}{l}\text { BV421 } \\
\text { FITC }\end{array}$ & & \\
\hline & $\begin{array}{l}\text { FITC } \\
\text { PerCP-Cy5.5 }\end{array}$ & & \\
\hline \multirow[t]{3}{*}{ CD4 } & APC- $\mathrm{H} 7$ & GK1.5 & $\mathrm{BD}$ \\
\hline & Biotin & H129.19 & BD \\
\hline & FITC & & \\
\hline \multirow[t]{4}{*}{ CD8a } & FITC & $53-6.7$ & $\mathrm{BD}$ \\
\hline & PE & & \\
\hline & PerCP-Сy5.5 & & \\
\hline & V450 & & \\
\hline \multirow[t]{4}{*}{ CD11b } & APC-Cy7 & $\mathrm{M} 1 / 70$ & $B D$ \\
\hline & BV421 & & \\
\hline & FITC & & \\
\hline & PE-Cy7 & & Ebioscience \\
\hline \multirow[t]{2}{*}{ CD11c } & FITC & HL3 & \\
\hline & PE-Cy7 & N418 & Ebioscience \\
\hline CD16/32 & Purified & $2.4 \mathrm{G} 2$ & $\mathrm{BD}$ \\
\hline \multirow[t]{4}{*}{ CD19 } & APC-Сy7 & 1D3 & $\mathrm{BD}$ \\
\hline & APC-Efluor780 & & Ebioscience \\
\hline & FITC & & \\
\hline & PerCP-Сy5.5 & & \\
\hline CD21/35 & APC & $7 \mathrm{G} 6$ & $\mathrm{BD}$ \\
\hline CD23 & PE-Cy7 & B3B4 & Ebioscience \\
\hline \multirow[t]{2}{*}{ CD25 } & $\mathrm{APC}$ & $3 \mathrm{C} 7$ & \\
\hline & PE-Cy7 & PC61.5 & Ebioscience \\
\hline CD31 & APC & 390 & Ebioscience \\
\hline CD44 & $\begin{array}{l}\text { PE } \\
\text { PE-Cy7 }\end{array}$ & IM7 & $\mathrm{BD}$ \\
\hline CD45R & BV421 & RA3-6B2 & $\mathrm{BD}$ \\
\hline CD62L & $\begin{array}{l}\text { FITC } \\
\text { APC }\end{array}$ & MEL -14 & $\mathrm{BD}$ \\
\hline CD68 & FITC & FA-11 & BioLegend \\
\hline \multirow[t]{2}{*}{ CD69 } & $\mathrm{PE}$ & $\mathrm{H} 1.2 \mathrm{~F} 3$ & \\
\hline & PE-Cy7 & & Ebioscience \\
\hline \multirow[t]{2}{*}{ FoxP3 } & A488 & MF23 & $\mathrm{BD}$ \\
\hline & PE & FJK-16s & Ebioscience \\
\hline \multirow[t]{2}{*}{$\lg D$} & PE & $11.26 .2 \mathrm{ca}$ & BD \\
\hline & $\begin{array}{l}\text { PE-Cy7 } \\
\text { PerCP-Efluor710 }\end{array}$ & $11-26 c$ & Ebioscience \\
\hline Igk & FITC & 187.1 & $\mathrm{BD}$ \\
\hline $\lg \lambda$ & FITC & R26-46 & $\mathrm{BD}$ \\
\hline \multirow[t]{2}{*}{$\lg M$} & $\begin{array}{l}\text { APC } \\
\text { APC-Efluor780 }\end{array}$ & $\mathrm{II} / 41$ & Ebioscience \\
\hline & Efluor450 & & \\
\hline \multirow[t]{2}{*}{ Ly6C } & A488 & ER-MP20 & AbD Serotec \\
\hline & PerCP-Cy5.5 & HK1.4 & Ebioscience \\
\hline \multirow[t]{4}{*}{ Ly6G } & APC-Cy 7 & $1 \mathrm{~A} 8$ & $\mathrm{BD}$ \\
\hline & BV421 & & \\
\hline & PE & & \\
\hline & PE-Cy7 & & \\
\hline NK1.1 & FITC & PK136 & Ebioscience \\
\hline TER-119 & FITC & TER-119 & $\mathrm{BD}$ \\
\hline
\end{tabular}


Tryptophan restriction arrests B cell development and enhances microbial diversity in WT and $E r c c 1^{-1 / 47}$ mice

Supplemental Table 2. Body weights, organ weights, and cellularity of various immune organs from young and aged WT and $E r c c 1^{-/ L Y}$ mice.

\begin{tabular}{|c|c|c|c|c|}
\hline & 16-wk WT & 18-mo WT & 6-wk Ercc1 ${ }^{-1 / \Delta 7}$ & 16-wk Ercc1 ${ }^{-1 \Delta 7}$ \\
\hline Body weight (g) & $22 \pm 0.91$ & $30 \pm 1.0^{* \star *}$ & $14 \pm 0.40$ & $16 \pm 0.50^{\star \star}$ \\
\hline Spleen weight (mg) & $70 \pm 7.8$ & $94 \pm 9.7^{\#}$ & $46 \pm 2.4$ & $45 \pm 1.9$ \\
\hline Relative spleen weight (\%) & $0.32 \pm 0.023$ & $0.32 \pm 0.032$ & $0.33 \pm 0.013$ & $0.27 \pm 0.010^{\star *}$ \\
\hline Thymus weight (mg) & $73 \pm 18$ & $49 \pm 9.0$ & $62 \pm 1.1$ & $28 \pm 2.8^{* \star \star}$ \\
\hline Relative thymus weight (\%) & $0.34 \pm 0.084$ & $0.16 \pm 0.026$ & $0.44 \pm 0.011$ & $0.17 \pm 0.021^{* * *}$ \\
\hline BM count $\left(\times 10^{6}\right)$ & $211 \pm 44$ & $230 \pm 27$ & $97 \pm 13$ & $127 \pm 12$ \\
\hline Thymus count $\left(\times 10^{6}\right)$ & $55 \pm 4.0$ & $30 \pm 4.0^{* *}$ & $59 \pm 4.8$ & $35 \pm 2.0$ ** \\
\hline Spleen count $\left(\times 10^{6}\right)$ & $140 \pm 21$ & $161 \pm 24$ & $101 \pm 4.1$ & $96 \pm 6.7$ \\
\hline MLN count $\left(\times 10^{6}\right)$ & $16 \pm 3.1$ & $61 \pm 20^{\#}$ & $16 \pm 1.2$ & $13 \pm 1.4$ \\
\hline PP count $\left(\times 10^{6}\right)$ & $1.9 \pm 0.49$ & $3.2 \pm 1.7$ & $0.82 \pm 0.25$ & $0.65 \pm 0.16$ \\
\hline PC count $\left(\times 10^{6}\right)$ & $5.9 \pm 1.5$ & $17 \pm 4.7^{\#}$ & $1.9 \pm 0.31$ & $2.7 \pm 0.09^{\#}$ \\
\hline
\end{tabular}

Mean (relative) weights or cell counts $\left(\times 10^{6}\right.$ cells) \pm SEM. BM count based on sternum, iliac crests, femurs plus tibias. $\mathrm{BM}=$ bone marrow; $\mathrm{MLN}=$ mesenteric lymph nodes; $\mathrm{PP}=$ Peyer's patches; $\mathrm{PC}=$ peritoneal cavity. Data represent $n=6$ mice per group. ${ }^{\#}=p<0.1 ;{ }^{*}=p<0.05 ;{ }^{* *}=p<0.01 ;{ }^{* * *}=p<0.001$. 
A

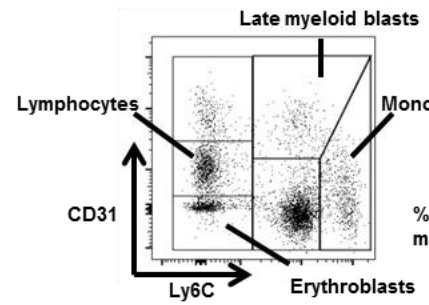

C

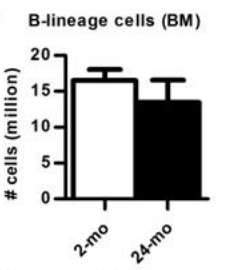

D Late myeloid blast cells (BM) Neutrophils (BM)
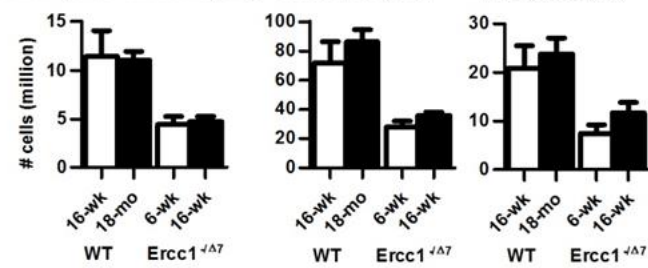

WT Ercc1

E
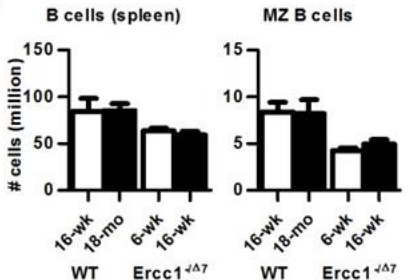

F B cells (spleen)

MZ B cells

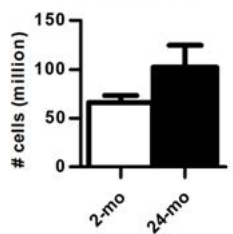

G

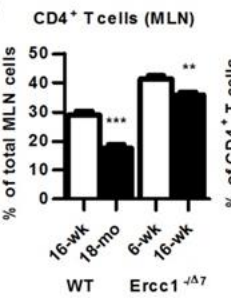

Follicular B cells
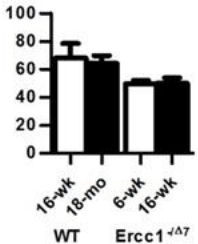

Follicular B cells

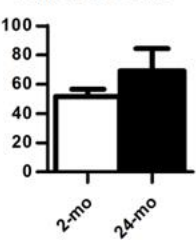

B

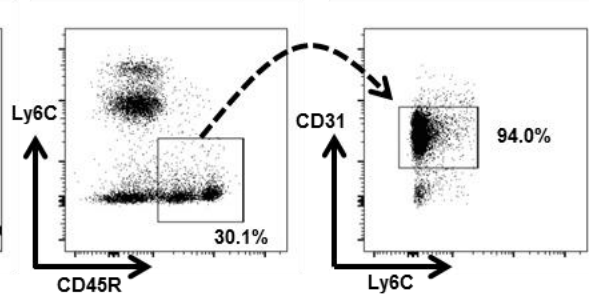

Mature B cells

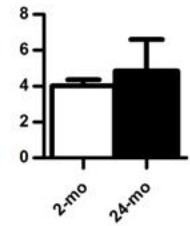

Erythroblasts (BM) Neutrophils (spleen) Monocytes (spleen)
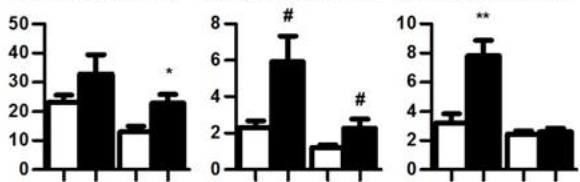

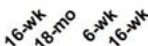
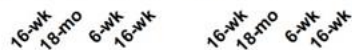

WT Ercc1 ${ }^{\text {A }}$ T

WT Ercc1 $1^{+\Delta 7}$

WT Ercc1 $1 \Delta 7$

B cells (MLN)

B cells (PP)
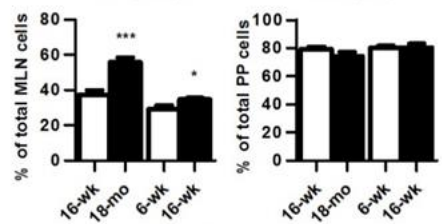

WT Erce1 ${ }^{\text {ta }}$

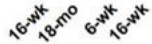

WT Ercc1 ${ }^{+\Delta}$
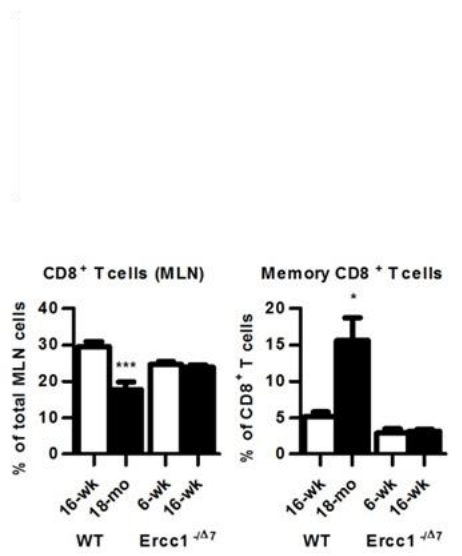

Supplemental Figure 1 (see legend next page). 

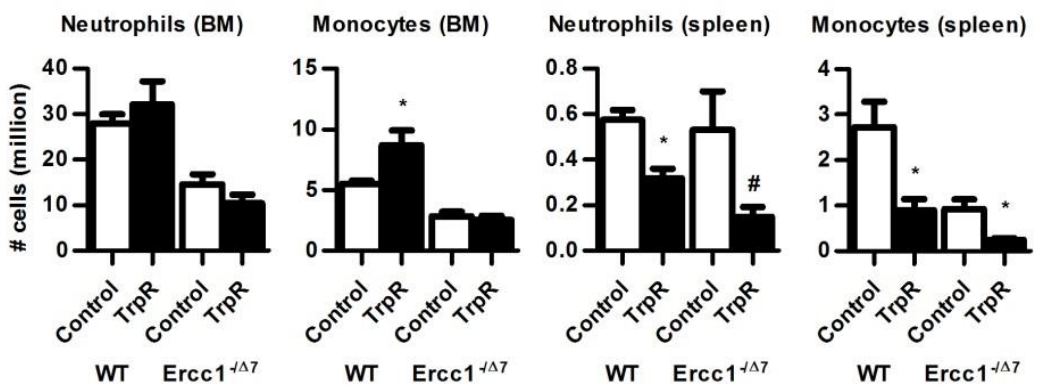

WT Ercc1 $1^{+\Delta 7}$

WT Ercc1 $1 \Delta 7$

Supplemental Figure 2. Effects of dietary tryptophan restriction on myeloid cell numbers in bone marrow (BM) and spleen. Mean numbers of $C D 11 b^{+} L y 6 G^{+}$neutrophils and Ly6C ${ }^{\text {hi }} C D 31^{-1 / 0}$ monocytes in $\mathrm{BM}$, and $\mathrm{CD} 11 \mathrm{~b}^{+}$Ly6 $\mathrm{C}^{\text {int }} \mathrm{CD} 68^{-1 / 0}$ neutrophils and $\mathrm{CD} 11 \mathrm{~b}^{+} \mathrm{CD} 68^{+}$monocytes in spleen were determined by flow cytometry. $\#=p<0.1 ;{ }^{*}=p<0.05$. Data represent the mean \pm SEM of $n=3-4$ mice per group. TrpR $=$ tryptophan restriction.

Supplemental Figure 1 (previous page). Myeloid cell and erythroblast frequencies in bone marrow (BM) and spleen, B cells in periphery, and T cells in mesenteric lymph nodes (MLN) change similar with aging in WT mice and Ercc1 ${ }^{-1 \Delta 7}$ mice. A) Flow cytometric analysis of BM composition by Ly6C and CD31. Lymphocytes are further gated for CD45R. In gray the isotype control, and in red CD45R staining. B) Combined flow cytometric analysis of B cells and Ly6C/CD31 in the BM. Ly6C-CD45R+ cells are further gated for Ly6C/CD31. C) Mean absolute numbers of total B-lineage cells, B cell precursors, immature, and mature $\mathrm{B}$ cells in 2-month-old or 24-month-old $\mathrm{C} 57 \mathrm{BI} / 6 \mathrm{~J}$ x FVB F1 mice were determined by flow cytometry. BM counts based on two femurs and two tibias. D) Mean numbers of $\mathrm{Ly}_{6 \mathrm{C}}{ }^{+} \mathrm{CD} 31^{+}$late myeloid blasts, CD $11 b^{+}$Ly6G $\mathrm{G}^{+}$neutrophils, Ly6C ${ }^{\mathrm{hi}} \mathrm{CD} 31^{-1 / 0}$ monocytes, Ly6C CD31 ${ }^{-}$erythroblasts in $\mathrm{BM}$, and $\mathrm{CD} 11 \mathrm{~b}^{+}$Ly6 $\mathrm{C}^{\text {int }} \mathrm{CD} 68^{-1 / 0}$ neutrophils and $\mathrm{CD} 11 \mathrm{~b}^{+} \mathrm{CD} 68^{+}$monocytes were determined by flow cytometry. $\mathrm{E}$ ) Mean absolute numbers of splenic B cells, MZ B cells, and follicular B cells, and mean frequencies of CD19 ${ }^{+}$ $B$ cells in MLN and PP. F) Mean absolute numbers of splenic B cells, MZ B cells, and follicular B cells in the spleen of 2-month-old or 24-month-old C57BI/6J x FVB F1 mice. Spleen count is based on a part of the spleen (approximately $80-90 \%$, based on spleen weights). G) Mean frequencies of CD4 ${ }^{+} \mathrm{T}$ cells and $\mathrm{CD}^{+} \mathrm{T}$ cells, and mean frequencies of memory $\mathrm{CD}^{+} \mathrm{T}$ cells $\left(\mathrm{CD} 44^{\mathrm{hi}} \mathrm{CD} 62 \mathrm{~L}^{-}\right)$, Treg $\left(\mathrm{FoxP}^{+}\right)$, and memory $\mathrm{CD}^{+} \mathrm{T}^{-}$

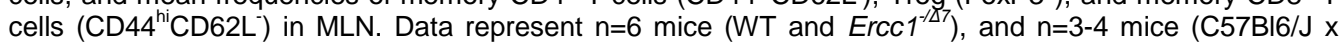
FVB F1 mice), expressed as mean \pm SEM. $\#=p<0.1 ;{ }^{*}=p<0.05 ;{ }^{* *}=p<0.01 ;{ }^{* * *}=p<0.001$. WT = wild-type C57BI6/J. 


\section{Chapter 8}

\section{Aged gut microbiota contribute to systemical inflammaging}

Floris Fransen ${ }^{1,2}$, Adriaan A. van Beek ${ }^{1,3}$, Theo Borghuis, ${ }^{1,2}$, Sahar El Aidy ${ }^{4}$, Floor Hugenholtz ${ }^{1,7}$, Christa van der Gaast-de Jongh ${ }^{5}$, Huub F.J. Savelkoul ${ }^{3}$, Marien I. De Jon$\mathrm{ge}^{5}$, Mark V. Boekschoten ${ }^{1,6}$, Hauke Smidt ${ }^{1,7}$, Marijke M. Faas ${ }^{2,8}$, Paul de Vos ${ }^{1,2}$

${ }^{1}$ Top Institute Food and Nutrition, Wageningen, The Netherlands; ${ }^{2}$ University of Groningen, University Medical Center Groningen, dept. Pathology and Medical Biology, Groningen, The Netherlands; ${ }^{3}$ Cell Biology and Immunology Group, Wageningen University, Wageningen, The Netherlands; ${ }^{4}$ Microbial Physiology, Groningen Biomolecular Sciences and Biotechnology Institute (GBB), University of Groningen, Nijenborgh 7, 9747, AG, Groningen, The Netherlands; ${ }^{5}$ Laboratory of Pediatric Infectious Diseases, Radboud University Medical Center, Nijmegen, The Netherlands; ${ }^{6}$ Nutrition, Metabolism \& Genomics Group, Division of Human Nutrition, Wageningen University, Wageningen, The Netherlands; ${ }^{7}$ Laboratory of Microbiology, Wageningen University, Wageningen, The Netherlands; ${ }^{8}$ University of Groningen, University Medical Center Groningen, Department of Obstetrics and Gynaecology, Groningen, The Netherlands 
Chapter 8

\section{ABSTRACT}

Advanced age is associated with chronic low-grade inflammation, which is usually referred to as inflammaging. Elderly are also known to have an altered gut microbiota composition. However, whether inflammaging is a cause or consequence of an altered gut microbiota composition is not clear. Here we show for the first time by transferring aged microbiota to young germ-free mice that certain bacterial species within the aged microbiota promote inflammaging. This effect was associated with lower levels of $A k$ kermansia and higher levels of TM7 bacteria and Proteobacteria in the aged microbiota after transfer. The aged microbiota promoted inflammation in the small intestine in the germ-free mice and enhanced leakage of inflammatory bacterial components into the circulation. As a consequence, the aged microbiota promoted increased $\mathrm{T}$ cell activation in the systemic compartment. 


\section{INTRODUCTION}

The gut microbiota is a highly complex and diverse community of bacteria that closely interacts with the epithelium and underlying immune cells in the gut [542]. The bacterial divisions that dominate the human gut microbiota are Firmicutes, Bacteroidetes, Actinobacteria, and Proteobacteria [543]. The dominance of these bacterial divisions is evolutionary conserved and has been confirmed in different mammalian species $[544,545]$. In recent years it has become clear that the gut microbiota has a major impact on the immune system, metabolism, and even behavior of the host [546]. Moreover, an imbalance in gut microbiota composition (dysbiosis) has been associated with several immunological, metabolic, and mental disorders [547]. However, for the majority of these diseases it remains unclear whether dysbiosis is a cause or consequence of the disease.

In adults the gut microbial community remains relatively stable [548]. However, a number of studies have shown that gut microbiota composition is different in the elderly. For example, it has been demonstrated that Firmicutes was dominant in the gut microbiota of young individuals, whereas Bacteroidetes was more prevalent in the gut microbiota in the elderly [549, 550]. Others found a decrease in anaerobes such as Bifidobacteria, but an increase in Enterobacteria, such as E. coli in the elderly [551, 552]. In people above 100 years old an increase in pathobionts was observed [107]. Also bacteria with anti-inflammatory properties such as Faecalibacterium prauznitzii were decreased in older individuals [553].

Concomitantly with microbiota changes, immunity becomes impaired in elderly [554]. Elderly are known to be more susceptible to infections and mount less effective immune responses after vaccination. Moreover, homeostasis between pro-inflammatory and regulatory responses is lost, which results in a state of low-grade chronic systemic inflammation [554]. The age-related chronic inflammation, which is called inflammaging, likely contributes to the pathology of several diseases typically associated with aging such as dementia, stroke, and cardiovascular diseases [555-557]. In addition, advanced age has been reported to increase intestinal permeability in rodents and non-human primates and may subsequently enhance translocation of luminal bacterial products and induce inflammation [558, 559].

Whether age-induced microbiota changes are associated with inflammaging is not entirely clear, but there are some indications that intestinal microbes are involved in this process [560]. To address the influence of the aged gut microbiota on the immune system, we transferred the gut microbiota from young or old conventional mice to germ-free mice. We demonstrate that the aged microbiota induced higher frequencies of several T helper cell subsets, in particular in the spleen. Moreover, expression of several inflammatory markers was elevated in the ileum after transferring microbiota of aged mice. Presumably translocation of bacterial components occurred, since the se- 
Chapter 8

rum after transfer of aged microbiota contained higher levels of immunostimulatory bacterial components. Finally, gut microbiota composition analysis revealed differences in abundance of bacterial species such as Akkermansia, TM7, and Proteobacteria, which are potentially involved in the increased inflammatory potential of the microbiota of aged mice.

\section{MATERIALS AND METHODS}

\section{Mice}

Young (7-10 weeks) or old (17 months) C57BL/6JRccHsd conventional female mice were purchased from a commercial supplier (Envigo, Horst, the Netherlands). Female germ-free mice of 12-14 weeks were obtained from a breeding colony at the animal facility of Radboud University Nijmegen Medical Centre (Nijmegen, the Netherlands). All animals were put on an autoclaved Rat/mouse maintenance V153X R/M-H diet (Ssniff, Soest, Germany) directly after weaning in the case of germ-free mice, or directly after arrival in the case of conventional mice. The mice were kept on this diet throughout the experiment. Conventional mice were housed in IVC cages and germfree mice were housed in germ-free isolators. All experiments were approved by the local ethical committee of the University of Groningen.

\section{Gut microbiota transfer}

After an acclimatization period of at least 4 weeks, feces were freshly collected from the conventional mice. Feces from the same group was pooled and mixed in PBS. Next, $200 \mu \mathrm{L}$ of $100 \mathrm{mg} / \mathrm{ml}$ of this mixture was given by oral gavage to germ-free mice of 12-14 weeks old. After transfer, recipient mice were individually housed in IVC cages for another 4 weeks.

\section{Organ and tissue collection}

Mice were sacrificed at the following ages: young conventional mice 16-19 weeks, old conventional mice 19-20 months, germ-free recipient mice 16-18 weeks, germ-free mice 13-15 weeks. Mice were anesthetized with isoflurane, bled, and sacrificed by cervical dislocation. Serum was collected and stored at $-80^{\circ} \mathrm{C}$. Colon content and a piece of terminal ileum were snap frozen in liquid nitrogen and stored at $-80^{\circ} \mathrm{C}$. In addition, spleen, Peyer's patches (PPs), and mesenteric lymph nodes (MLNs) were collected for FACS analysis. 


\section{Flow cytometry}

Single cell suspensions were obtained from spleen, PPs, and MLNs. Cells were stained with Fixable Viability Dye eFluor 506 (eBioscience, Vienna, Austria) for exclusion of dead cells. A-specific binding to $\mathrm{Fc}$ receptors was blocked by incubating the cells with anti-CD16/32 (clone 93, BioLegend, Uithoorn, the Netherlands) for 15 min on ice. For extracellular staining, cells were incubated with the desired mixture of antibodies for 30 min on ice. After washing, cells were fixed with FACS lysing solution (BD Biosciences, Breda, the Netherlands). For intracellular staining, fixed cells were permeabilized with PERM (eBioscience, Vienna, Austria) and subsequently stained with the desired antibodies for $30 \mathrm{~min}$ on ice. For identification of the different $\mathrm{T}$ helper cell subsets, cells were stained with antibodies against: CD3e (clone 17A2), CD4 (clone GK1.5), T-bet (clone 4B10), RORyt (clone B2D), Gata-3 (clone TWAJ), CD25 (clone PC61), and Foxp3 (clone FJK-16S). Appropriate isotype controls were used to determine specificity of the staining. Samples were acquired with the FACSVerse (BD Biosciences, Breda) and analyzed with FlowJo software (FlowJo, LLC, Oregon, USA).

\section{Transcriptome Microarray}

A piece of terminal ileum from each mouse was snap frozen in liquid nitrogen and stored afterwards at $-80^{\circ} \mathrm{C}$. From these samples RNA was isolated with the RNeasy kit (Qiagen, Valencia, CA, USA). Quantity of RNA was measured with the ND-1000 (NanoDrop Technologies, Thermo Fisher Scientific, Breda, the Netherlands) and quality of RNA was assessed with the Bioanalyzer 2100 (Agilent, Santa Clara, CA, USA). Total RNA (100 ng) was labeled utilizing the GeneChip WT plus reagent kit (Affymetrix, Santa Clara, CA, USA). After labeling, samples were hybridized to Affymetrix GeneChip Mouse Gene 1.1 ST arrays. An Affymetrix GeneTitan Instrument was used for hybridization, washing, and scanning of the array plates. Bioconductor packages integrated in an online pipeline were used for quality control of the data $[561,562]$. Probe sets were redefined using current genome information [563]. Probes were reorganized based on the Entrez Gene database (remapped CDF v19). Robust Multi-array Analysis preprocessing algorithm available in the Bioconductor library affyPLM [564] was used to obtain normalized expression estimates from the raw intensity values.

\section{HEK293 TLR2/TLR4 assay}

Human Embryonic Kidney 293 cells stably transfected with mouse TLR2/CD14 or TLR4/MD-2/CD14 and the secreted embryonic alkaline phosphatase reporter (SEAP) coupled to the NF-kB/AP-1 promoter were purchased from Invivogen (San Diego, CA, USA). Every cell line was grown at $37^{\circ} \mathrm{C}, 5 \% \mathrm{CO}_{2}$ in DMEM medium (Lonza B.V., Basel, Switzerland), supplemented with $4.5 \mathrm{~g} / \mathrm{l}$ glucose, $10 \%$ heat-inactivated FBS, $2 \mathrm{mM}$ 
Chapter 8

L-glutamine, $50 \mathrm{U} / \mathrm{ml}$ penicillin, $50 \mathrm{mg} / \mathrm{ml}$ streptomycin, and $100 \mathrm{mg} / \mathrm{ml}$ Normocin. After 2 passages, the cells were cultured in the presence of HEK-Blue selection medium (Invivogen, San Diego, CA, USA) in order to maintain the transfected constructs. Cells were stimulated with $2.5 \%$ mouse serum for 20 hours at $37^{\circ} \mathrm{C}, 5 \% \mathrm{CO}_{2}$. Next, $20 \mu \mathrm{l}$ medium from each well was aliquoted and mixed with $180 \mu$ QUANTI-Blue reagent (Invivogen, San Diego, CA, USA). After incubation at $37^{\circ} \mathrm{C}$ for 2 hours, OD at $650 \mathrm{~nm}$ was measured with a microplate absorbance spectrophotometer (Bio-Rad Laboratories, Veenendaal, the Netherlands).

\section{Microbiota analysis}

Fresh feces samples obtained just after defecation were collected from all mice at different time points during the experiment. In addition, colonic content samples from these mice were collected at the end of the experiment. All samples were snap frozen in liquid nitrogen and stored at $-80^{\circ} \mathrm{C}$. These samples were used for $16 \mathrm{~S}$ rRNA gene analysis for microbiota profiling with barcoded amplicons from the V1-V2 region of $16 \mathrm{~S}$ rRNA genes generated using a 2-step PCR strategy that reduces the impact of barcoded primers on the outcome of microbial profiling [565]. DNA extraction was performed using a combination of the bead-beating-plus column method and the Maxwell 16 Tissue LEV Total RNA purification kit (Promega, Leiden, The Netherlands). Beating of the fecal pellets took place as described before [520], but with STAR (Stool transport and recovery) buffer (Roche, Basel Switzerland). $250 \mu$ l supernatant after centrifugation was taken for the Maxwell 16 Tissue LEV Total RNA Purification Kit and the DNA was eluted in $50 \mu \mathrm{l}$ DNAse free water. Twenty nanograms of DNA was used for the amplification of the 16S rRNA gene with primers 27F-DegS and 338R I + 338R II for 25 cycles as described before [521], only primers had a UniTag linkers attached; UniTag I (forward) and II (reverses) (I - GAGCCGTAGCCAGTCTGC; II GCCGTGACCGTGACATCG). The first PCR was performed in a total volume of $50 \mu \mathrm{l}$ containing $1 \times \mathrm{HF}$ buffer (Finnzymes, Vantaa, Finland), $1 \mu \mathrm{l}$ dNTP Mix (10 mM; Promega, Leiden, The Netherlands), $1 \mathrm{U}$ of Phusion® Hot Start II High-Fidelity DNA polymerase (Finnzymes Vantaa, Finland), $500 \mathrm{nM}$ of the 27F-DegS primer [521, 566] that was appended with Universal Tag (UniTag) 1 at the 5' end, $500 \mathrm{nM}$ of an equimolar mix of two reverse primers, 338R I and II [566] based on three previously published probes EUB $338 \mathrm{I}$, II and III [521], that were 5'-extended with UniTag 2, and 0.2-0.4 $\mathrm{ng} / \mu \mathrm{l}$ of template DNA. The sequence of the UniTags were selected to have a GC content of $\sim 66 \%$ and a minimal tendency to form secondary structures, including hairpin loops, heterodimers, and homodimers as assessed by the IDTDNA Oligoanalyzer 3.1 (Integrated DNA Technologies). Moreover, sequences were selected that had no matches in 16S rRNA gene databases (based on results of the 'TestProbe' tool offered by the SILVA rRNA database project [567] using the SSU r117 database), and 
no prefect matches in genome databases with the Primer-BLAST tool (http://www.ncbi.nlm.nih.gov/tools/primer-blast/). The size of the PCR products ( 375 bp) was confirmed by gel electrophoresis using $5 \mu \mathrm{L}$ of the amplification reaction mixture on a $1 \%(w / v)$ agarose gel containing $1 \times$ SYBR® Safe (Invitrogen, Thermo Fisher Scientific, Waltham, MA, USA). Five $\mu \mathrm{L}$ of these PCR products were taken to add adaptors and an 8-nt sample-specific barcode in an additional 5 cycle PCR amplification. This second PCR was performed in a total volume of $100 \mu$ l containing $1 \times \mathrm{HF}$ buffer, dNTP Mix $2 \mathrm{U}$ of Phusion® Hot Start II High-Fidelity DNA polymerase, $500 \mathrm{nM}$ of a forward and reverse primer equivalent to the Unitag1 and UniTag2 sequences, respectively, that were each appended with an $8 \mathrm{nt}$ sample specific barcode at the 5 ' end (G. Hermes and J. Ramiro-Garcia, et al In preparation). PCR products were purified with the magnetic beads (MagBio, London, UK) according to the HighPrepTM protocol of the manufactures instructions using $20 \mu \mathrm{L}$ Nuclease Free Water (Promega Leiden, The Netherlands) and quantified using the Qubit (Life Technologies, Bleiswijk, The Netherlands). Purified PCR products were mixed in approximately equimolar amounts and concentrated by the magnetic beads as the purification before. Purified amplicon pools were 250 bp paired-end sequenced using Illumina Miseq (GATCBiotech, Konstanz, Germany).

The Illumina Miseq data analysis was carried out with a workflow employing the Quantitative Insights Into Microbial Ecology (QIIME) pipeline [522] and a set of in-house scripts as described before for Illumina Hiseq 16S rRNA gene sequences (G. Hermes and J. Ramiro-Garcia, et al In preparation). The set of in-house scripts processed the reads as follows: reads were filtered for not matching barcodes; otu picking and chimera removal was done via matching the sequences to the Silva 111 database, with only one mismatch allowed, and a biom and with clustalw a multiple alignment and phylogenetic tree file was generated. Further outputs were generated via Qiime, such as filtered reads per sample, PD whole tree diversity measurements and the level 1 to 6 taxonomic distributions with relative abundances.

\section{Statistics}

Flow cytometry data and HEK293 TLR assay data are expressed as means, error bars represent standard error of the mean (SEM). To verify whether data were normally distributed the Kolmogorov-Smirnov test was performed. In cases where data were not normally distributed, data were log transformed before analysis. For comparing 2 groups the unpaired two-tailed Student's T test was used. For comparing more than 2 groups with each other, one way ANOVA was performed followed by the Bonferroni test to compare specific groups. P-values below 0.05 were considered significant. All tests were performed with Graphpad software (Prism, La Jolla, CA, USA). 
Chapter 8

Differentially expressed probe sets were identified using linear models, applying moderated T-statistics that implemented empirical Bayes regularization of SEs [568]. A Bayesian hierarchical model was used to define an intensity-based moderated Tstatistic, which takes into account the degree of independence of variances relative to the degree of identity and the relationship between variance and signal intensity [569]. Statistical tests for gut microbiota composition were performed using $\mathrm{R}$ and Calypso [570], where the count data were not normally distributed and variances between groups were not equal, the Mann-Whitney $U$ test was used.

\section{RESULTS}

\section{Microbiota of old mice enhances $\mathrm{CD4}^{+} \mathrm{T}$ cell differentiation in the spleen}

In order to investigate how aging influences the interplay between the gut microbiota and the immune system of the host, we transferred gut microbiota from young (11-14 weeks) or old (18 months) conventional mice to young germ-free mice (12-14 weeks). Four weeks later the mice were sacrificed and the frequency of the different CD4 ${ }^{+} \mathrm{T}$ helper (Th) subsets were identified in the Peyer's patches (PP), mesenteric lymph nodes (MLN), and the spleen. Conventional mice, aged 11-14 weeks, or 18 months served as control.

In conventional mice, a higher frequency of Th2 cells was found in the spleen of old mice compared to young mice (Figure 1A). This enhanced Th2 frequency could be induced by transfer of the old microbiota to young germ-free mice and was not observed when young microbiota was transferred. Also the high Treg (Figure 1B) and Th1 (Figure 1C) numbers in spleens of old conventional mice could be induced by transfer of old microbiota. Germ-free mice, which received the old microbiota, had a higher frequency of splenic Tregs (Figure 1B) and Th1 cells (Figure 1C) than germfree mice which received the young microbiota. No differences were observed in Th17 cells (data not shown). Furthermore, in the PPs and MLNs no differences were observed in Th frequencies (data not shown), except for Th1 cells in PPs, which were significantly higher in germ-free mice after transfer of the old microbiota (Figure 1D) as compared to germ-free mice after transfer of microbiota of young mice. In conclusion, the old microbiota enhanced $\mathrm{CD} 4^{+} \mathrm{T}$ cell differentiation of several Th subsets, in particular in the systemic compartment. 
A

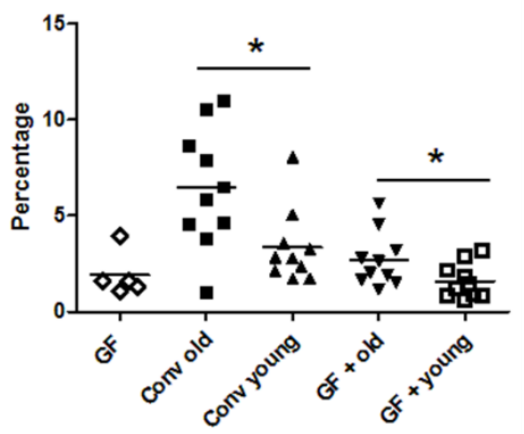

C

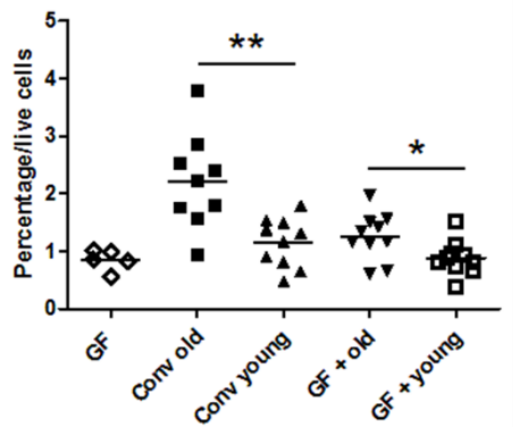

B

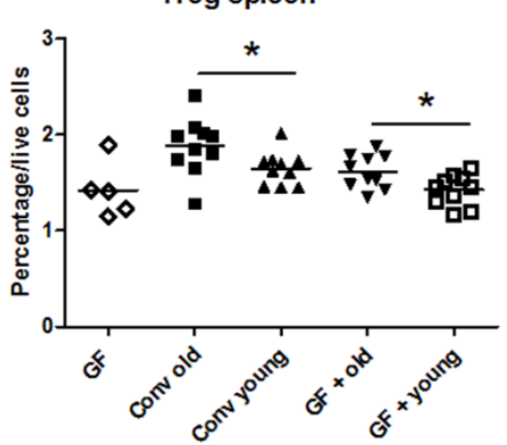

D

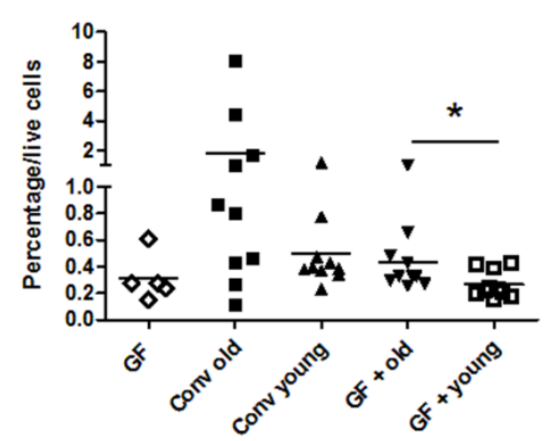

Figure 1. Old microbiota induces higher frequencies of $T$ helper subsets in the spleen. Spleen, MLN, and PP CD4 ${ }^{+} \mathrm{T}$ cell populations were analyzed with flow cytometry after isolation from young or old conventional (conv) mice ( $n=10$ ), germ-free (GF) recipient mice of young or old microbiota $(n=10)$, and GF control mice $(n=5)$. (A) Percentage of splenic CD4 ${ }^{+}$T cells expressing GATA-3 (Th2). (B) Percentage among total live cells of splenic CD4 ${ }^{+}$T cells expressing CD25 and Foxp3 (Treg). (C) Percentage among total live cells of splenic $\mathrm{CD4}^{+} \mathrm{T}$ cells expressing T-bet (Th1). (D) Percentage among total live PP cells of $\mathrm{CD4}^{+} \mathrm{T}$ cells expressing T-bet (Th1). All data are expressed as means. * indicates $\mathrm{P}<0.05$, ${ }^{*}$ indicates $\mathrm{P}<0.01$.

\section{Microbiota of old mice upregulates inflammation-associated immune pathways in the ileum}

To study the effect of the microbiota on the host in an unbiased manner we performed genome-wide gene expression analysis of the ileum with microarray. Genes that were significantly higher expressed in the ileum of old conventional mice compared to young conventional mice were analyzed with the STRING database [571]. We identified a large cluster of genes involved in the immune response that were upregulated in the ileum of old conventional mice (Figure 2A). The function of these genes included antigen processing and presentation, activation of the complement pathway, recognition of microbe-associated molecular patterns (MAMP), and migration of $B$ cells. TNF$\alpha$ was in the center of this network, which might suggest that TNF- $\alpha$ plays an important role in these processes. 


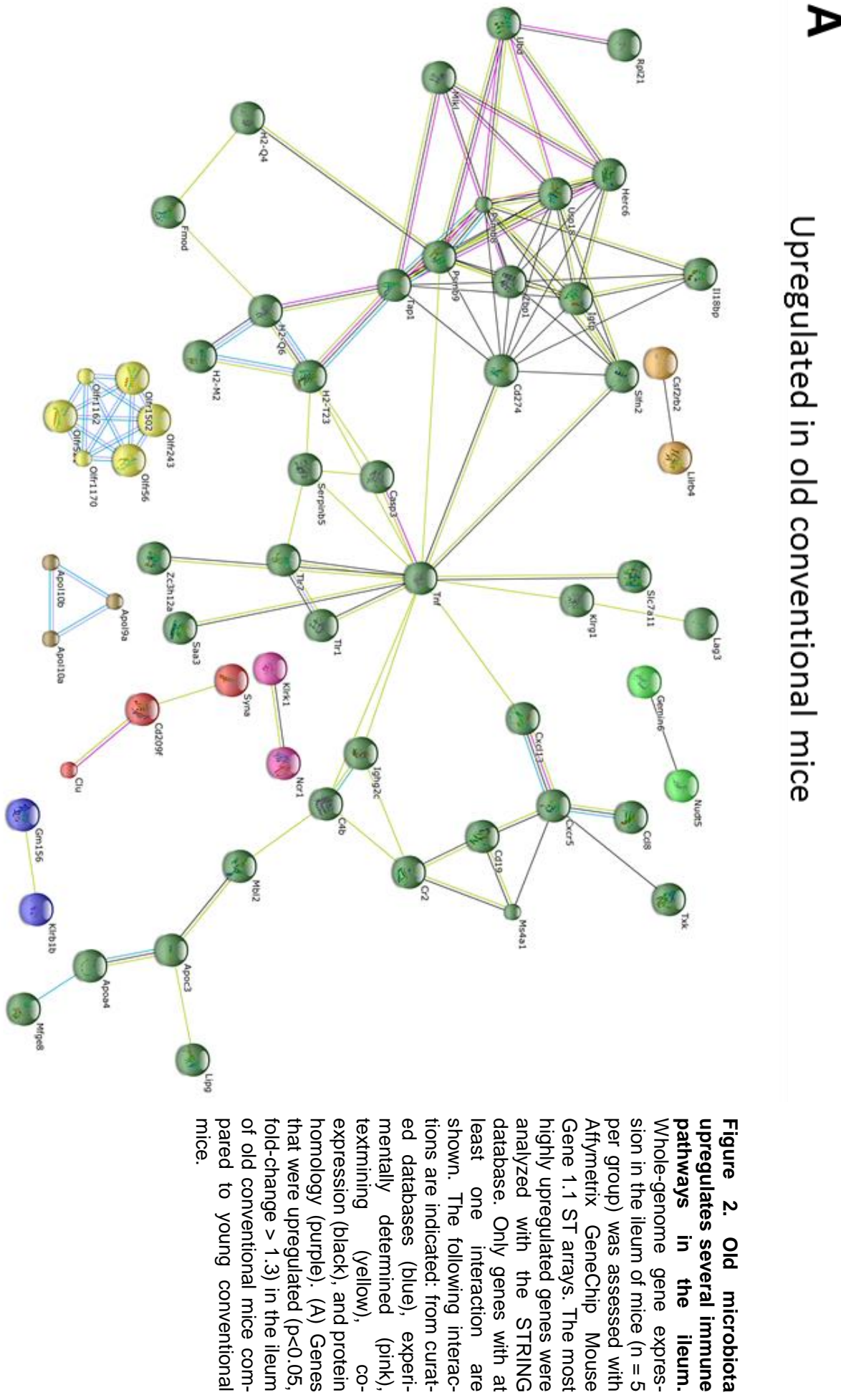



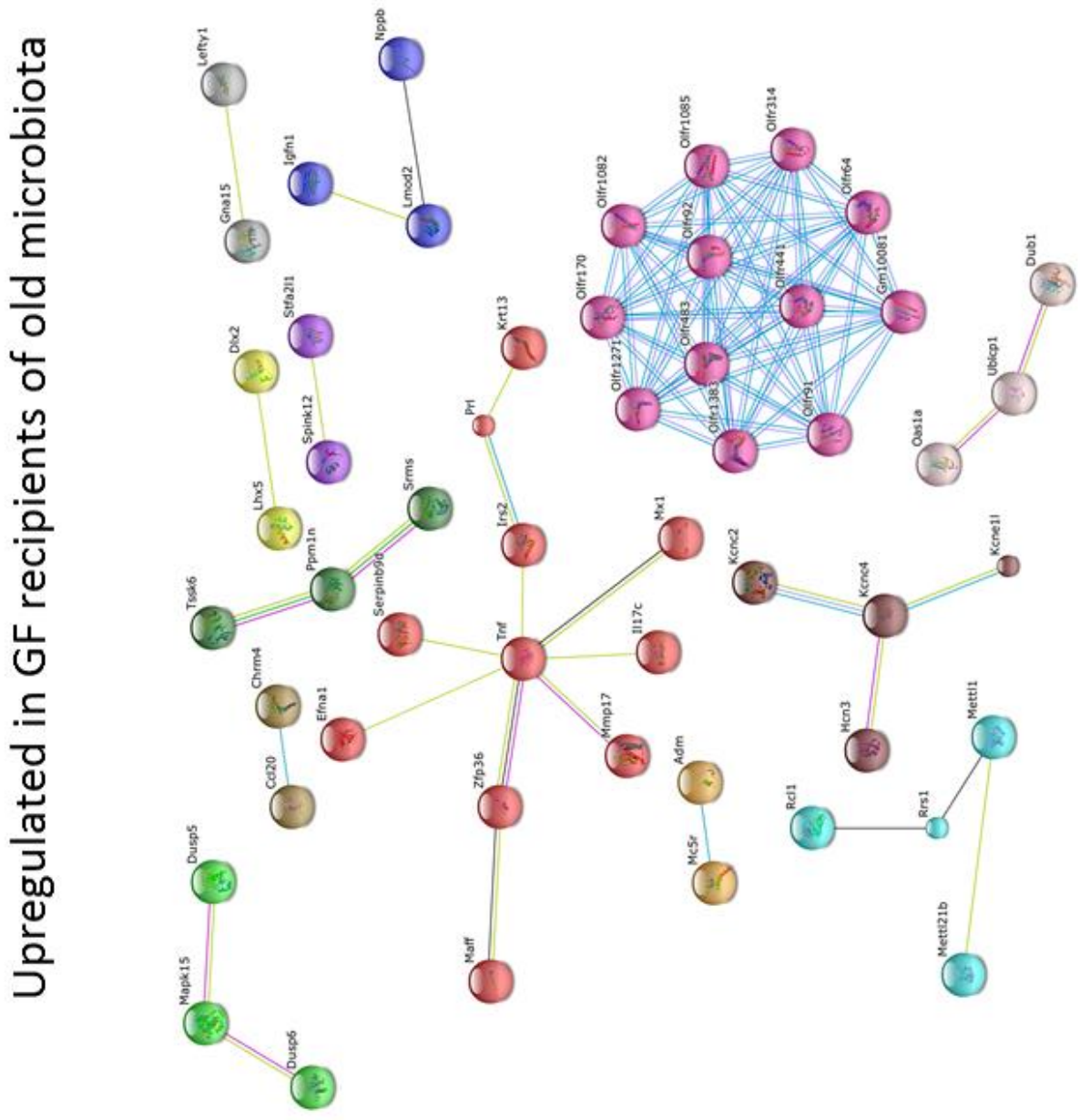

อง

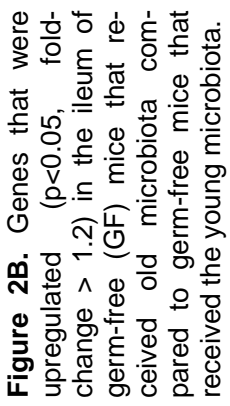




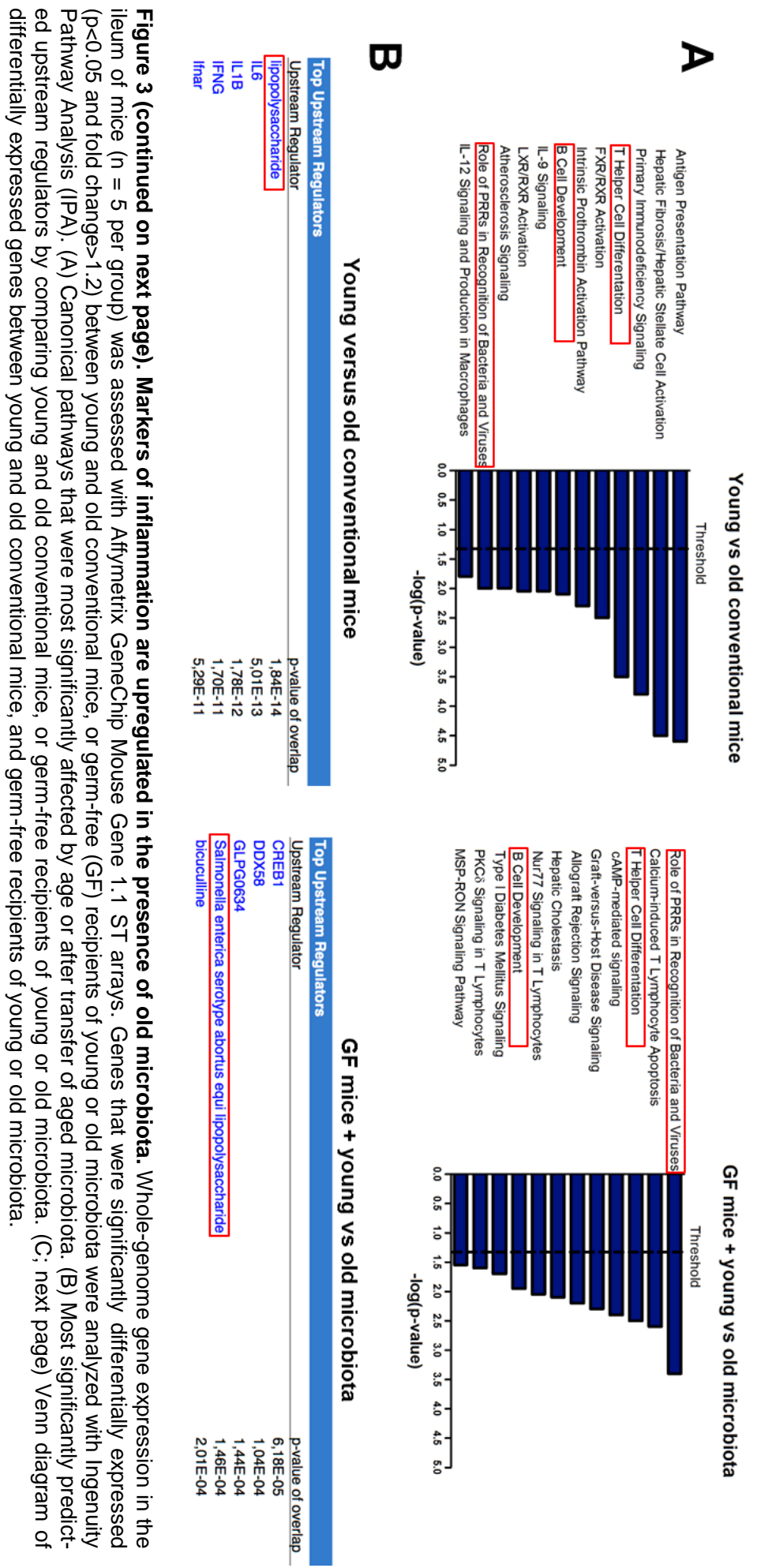



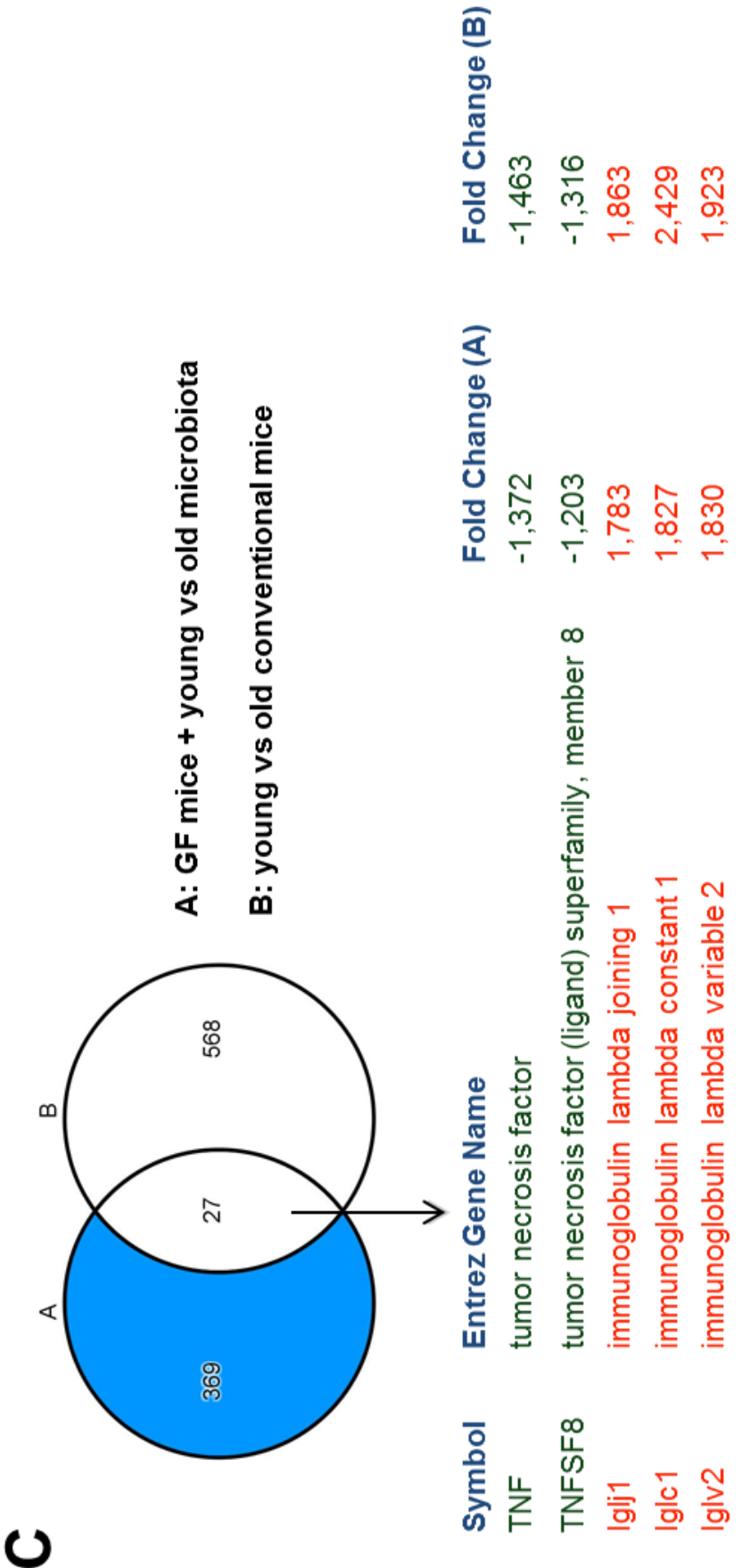
Chapter 8

Also genes that were significantly higher expressed in germ-free mice that received the old microbiota compared to recipients of young microbiota were analyzed with the STRING database. Also here we identified a cluster of genes with TNF- $\alpha$ in the center of the network (Figure 2B). These results might suggest that TNF- $\alpha$ production is specifically enhanced by the old microbiota.

\section{Identification of immune pathways specifically affected by old and young mi- crobiota}

Microarray data were further analyzed with Ingenuity Pathway Analysis (IPA), only focusing on genes that were significantly differentially expressed $(p<0.05$, fold change $>1.2$ or $<-1.2$ ) when comparing old versus young conventional mice, or germ-free recipient mice that received young versus old microbiota. Interestingly, we observed 3 canonical pathways that were significantly affected both in the conventional mice and in the germ-free recipient mice (Figure 3A). The canonical pathways 'role of PRRs in recognition of bacteria and viruses', 'T helper cell differentiation', and 'B cell development' were upregulated in old conventional mice compared to young conventional mice and also in germ-free recipients of old microbiota compared to recipients of young microbiota. Therefore, these pathways might be in particular influenced by the microbiota during aging.

Also predicted upstream regulators were identified with IPA. Upstream regulators are the upstream transcriptional regulators that potentially explain the observed gene expression differences in the dataset. The most significantly predicted upstream regulator that could cause the gene expression profile in old conventional mice in comparison to young conventional mice was lipopolysaccharide (LPS; Figure 3B). Importantly, LPS from Salmonella enterica was among the most significantly predicted upstream regulators of old microbiota after transfer to germ-free mice (Figure 3B). Thus, LPS is a component of the old microbiota that is possibly involved in mediating its effects on the immune system of the host.

To further identify the genes that were specifically influenced by the old microbiota, we compared the genes that were differentially expressed between young versus old conventional mice and germ-free recipients of young versus old microbiota (Figure 3C). We identified 27 genes that were differentially expressed in both datasets. This is list of genes was further narrowed down to genes that were up or down-regulated in both datasets and are known to play a role in the immune response. As mentioned above, TNF- $\alpha$ was upregulated both in old conventional mice and in germ-free recipients of old microbiota. Also, TNFSF8, which is the ligand for CD30, was more highly expressed in these groups of mice. On the other hand, several genes encoding for the 
lambda immunoglobulin light chain were more highly expressed both in young conventional mice and in germ-free recipients of young microbiota.
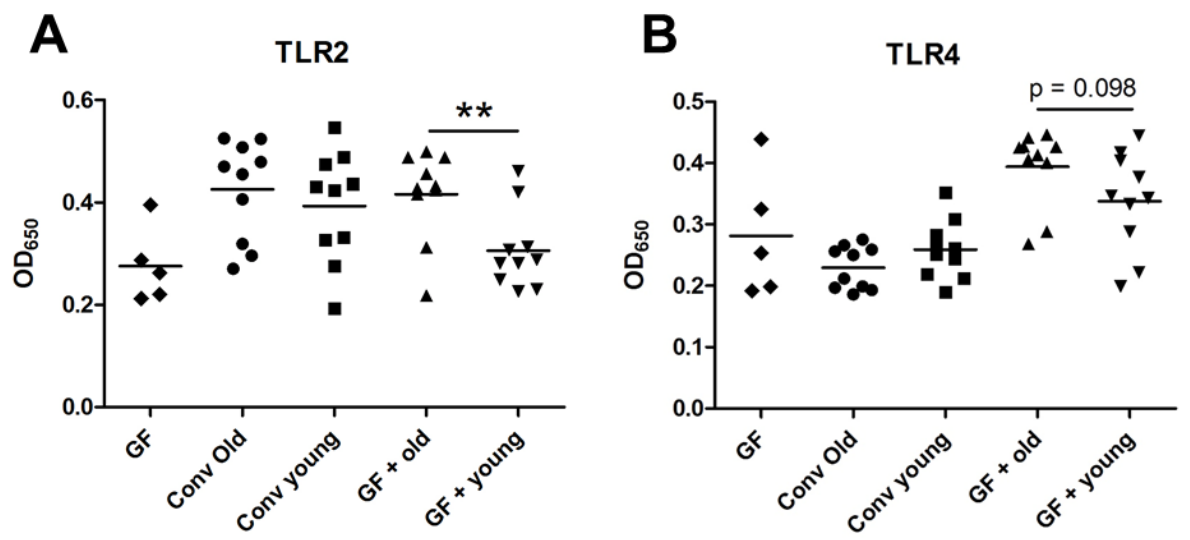

Figure 4. Transfer of old microbiota enhances inflammatory bacterial components in serum. Human Embryonic Kidney 293 cells transfected with mouse TLR2/CD14 (A) or mouse TLR4/MD-2/CD14 (B) were stimulated with $2.5 \%$ serum from young or old conventional (conv) mice $(n=10)$, germ-free (GF) recipient mice of young or old microbiota $(n=10)$, and GF control mice $(n=5)$. Activation of these receptors was measured with a secreted embryonic alkaline phosphatase reporter (SEAP) coupled to the NF-kB/AP-1 promoter. All data are expressed as means. ${ }^{* *}$ indicates $\mathrm{P}<0.01$.

\section{Increased leakage of bacterial components into systemic circulation after old microbiota transfer}

As LPS was a prominent predicted upstream regulator in the ileal IPA analysis, we investigated whether there were innate immune activating components in sera of animals receiving old microbiota. These components can possibly be transferred from the intestine by translocation of bacterial components due to a compromised intestinal barrier [572, 573]. To this end, we incubated the sera of these mice with HEK293 cells transfected with Toll-like receptor (TLR) 2 or TLR4. Activation of NF-kB was measured with a reporter gene. No differences were observed between old conventional mice and young conventional mice. However, the sera from germ-free mice, which had received old microbiota showed significantly higher activation of TLR2 compared to sera from recipients of young microbiota (Figure 4A). A similar trend was observed for TLR4 activation, although this difference did not reach statistical significance (Figure 4B). In summary, these data indicate that old microbiota transfer leads to increased translocation of inflammatory bacterial products into the circulation.

\section{Bacterial groups associated with increased inflammatory potential of old micro- biota}

Next, we investigated how the gut microbiota composition changes over time in the recipient mice. To this end the composition of the gut microbiota of the different exper- 
Chapter 8

imental groups was analyzed with 165 rDNA sequencing. From the germ-free recipient mice that received old or young microbiota, we analyzed feces 1 week after transfer or 4 weeks after transfer (Figure 5). We were particularly interested to see whether the gut microbiota evolves into a community similar to the donor, or whether it adapts to its host. Redundancy analysis (RDA) at the genus level confirmed that gut microbiota composition was different between old conventional mice and young conventional mice, since the samples separated into 2 distinct clusters (Figure 6A). The samples collected 1 week after transfer also separated into 2 different clusters, suggesting that the transfer of different gut microbiota communities also led to the establishment of different microbiota communities in the recipients. However, after 4 weeks gut microbiota composition in the recipient mice was most similar to the gut microbiota composition in young conventional mice. Moreover, at this time point the clusters of samples derived from the recipient mice were showing more overlap, which suggests gut microbiota composition of the 2 groups became more similar to each other compared to the first week time point. Together these results indicate that at 1 week the gut microbiota composition of the donor dictates the gut microbiota composition in the recipient, but at later time points the gut microbiota composition adapts to the host.

To look more specifically at the bacterial groups that were responsible for the observed differences in immune responses, we investigated which bacterial phyla had a significant difference in abundance (Figure 6B). Compared to young conventional mice, old conventional mice had higher abundance of Tenericutes, but lower abundance of Verrucomicrobia. Akkermansia is the only genus known to belong to the Verrucomicrobia phylum. Indeed we observed a similar difference in abundance for $A k$ kermansia (data not shown). In addition, age influenced the Firmicutes/Bacteroidetes ratio. Old conventional mice had more Bacteroidetes, but less Firmicutes compared to young conventional mice. Interestingly, 1 week after transfer of old microbiota, recipient-mice had significant less Verrucomicrobia than germ-free mice that received young microbiota (Figure 6B). There was also a difference in the Firmicutes/Bacteroidetes ratio, but surprisingly recipients of old microbiota had significantly less Bacteroidetes and more Firmicutes compared to recipients of young microbiota. Four weeks after transfer the differences at 1 week were no longer present. However, at this time point recipients of old microbiota had a higher abundance of TM7 and Proteobacteria (Figure 6B). The difference in Proteobacteria was likely due to a difference in abundance of Desulfovibrio, since this was the only Proteobacterium that was significantly more abundant at the genus level after transfer of old microbiota. In summary, a number of bacterial groups were identified that were affected by age, which included Akkermansia, TM7, and Proteobacteria. These bacterial groups are possibly involved in the increased inflammatory potential of the old microbiota. 

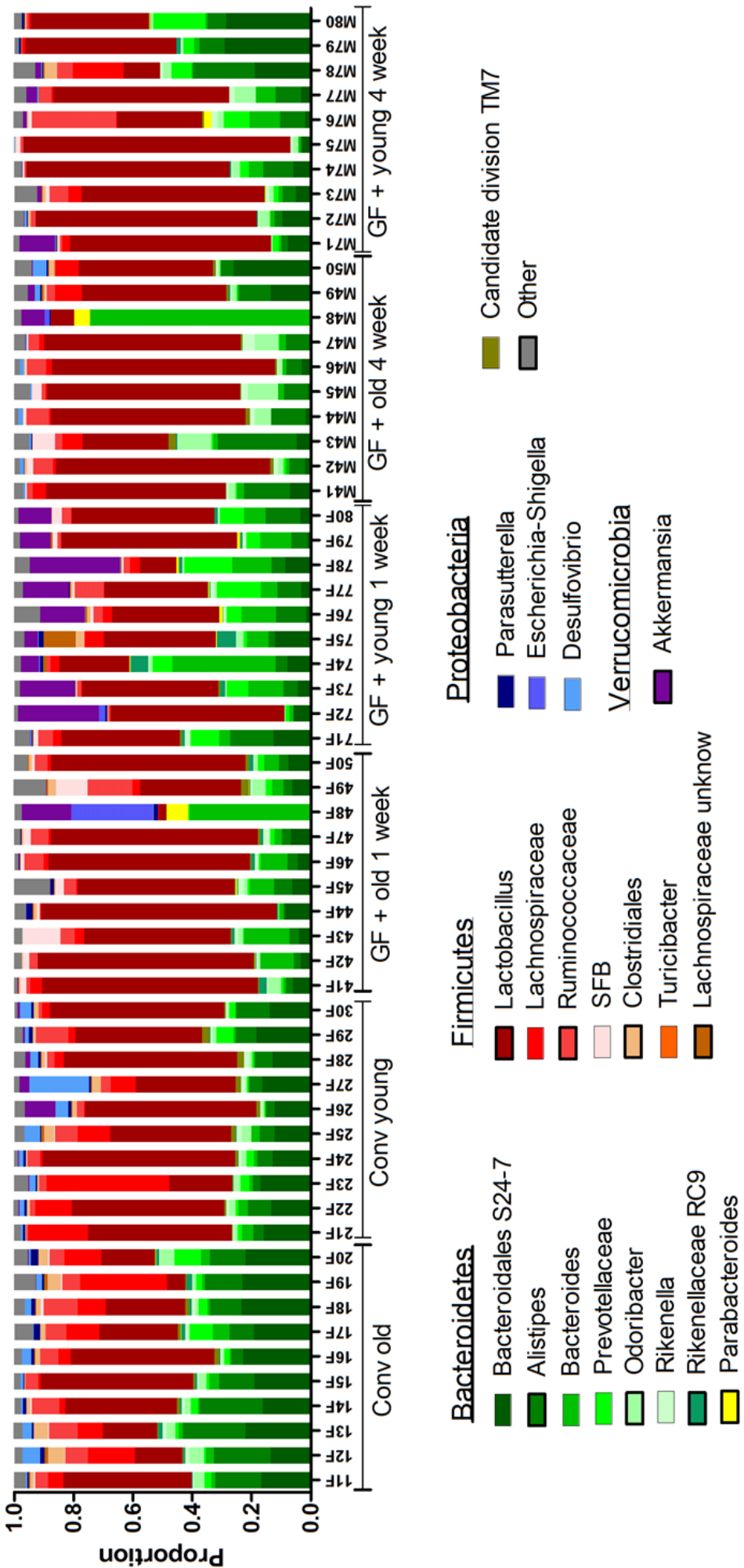

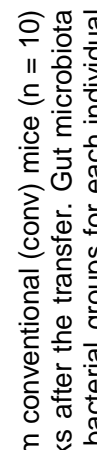

들 ڤै

广

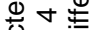

응

흉

要边

$\sum_{3}^{\infty}$

象

응으 흥

है

$\omega$

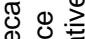

एᄂ.

هं

ह $\frac{\mathrm{o}}{2}=$

응 
Chapter 8
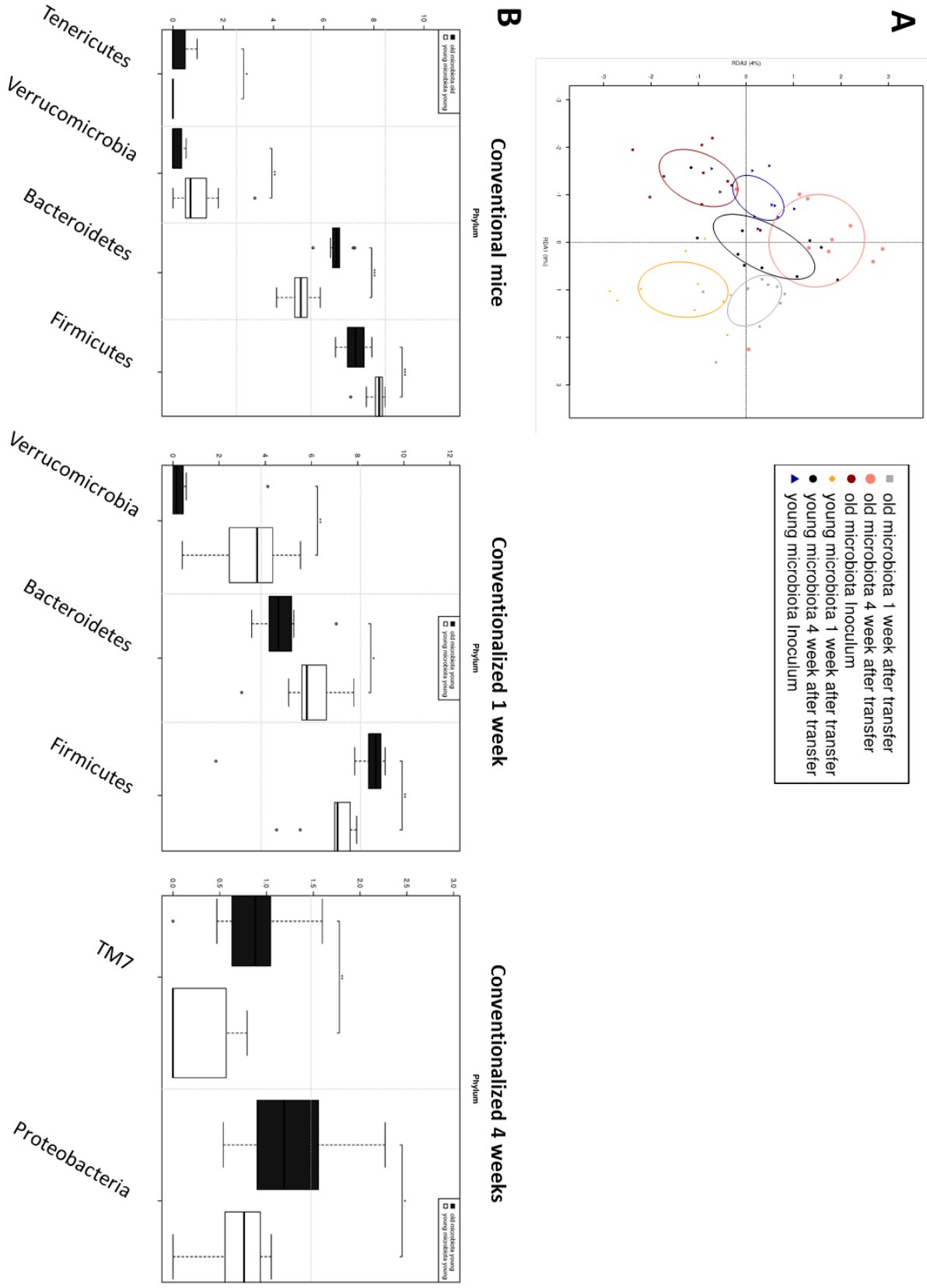

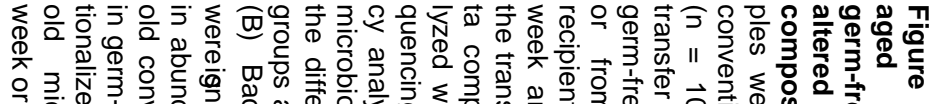

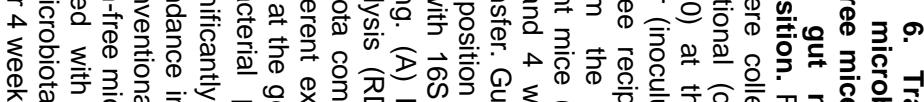

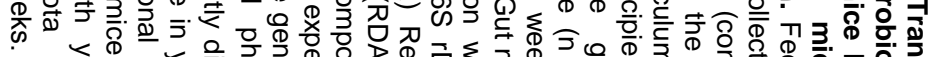
@ 仓 栗

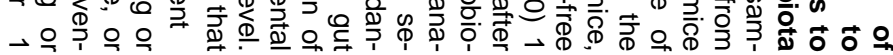




\section{DISCUSSION}

Several studies have demonstrated that aging is associated with an altered gut microbiota composition, inflammaging, and increased gut permeability [560]. However, whether the aged microbiota is a cause or consequence of inflammaging is not known. To the best of our knowledge, we are the first to show that some characteristics of this typical immunosenescence can be induced by microbiota of aged mice after transfer into young germ-free mice. Microorganisms responsible for this effect were found to be Akkermansia, TM7 bacteria and Proteobacteria. Our results suggest that in the presence of aged microbiota, TLR2- and 4-stimulating components were found in the circulation of recipients of old microbiota. This leads to increased tissue inflammation, which eventually results in enhanced $\mathrm{T}$ cell differentiation systemically. Interestingly, dysbiosis and a comprised intestinal barrier is also observed in several other disorders such as IBD and metabolic syndrome [574, 575].

Certain bacterial species colonizing the gut have been shown to induce a specific subset of Th cell. For example, segmented filamentous bacterium was found to specifically induce Th17 cells in the gut [576]. On the other hand, polysaccharide A (PSA) produced by the human symbiont Bacteroides fragilis was shown to promote expansion of IL-10-producing $\mathrm{T}_{\text {reg }}$ cells in a TLR2-dependent manner [577-579]. It has also been demonstrated that certain Clostridium species induce $\mathrm{T}_{\text {reg }}$ cells in the colon [580, 581]. In our study the aged microbiota did not promote differentiation of a specific Th cell subset. However, after transfer of aged microbiota to germ-free mice, we rather observed increased levels of several Th cell subsets. This effect was almost exclusively observed in the spleen, but not in the PPs or MLNs. These results do not suggest an association with any of the bacterial species mentioned above. An increased exposure of naïve $\mathrm{T}$ cells in the systemic compartment to bacterial compounds in general as a result of a reduced intestinal barrier seems a more likely explanation.

The transfer of old microbiota into young germ-free mice induced differential regulation of pathways including $\mathrm{T}$ cell differentiation, $\mathrm{B}$ cell development, and recognition of microbes by pattern recognition receptors. A central regulatory cytokine was TNF- $\alpha$, which was consistently upregulated by the old microbiota both in the conventional mice and germ-free recipient mice. TNF- $\alpha$ is well known for its role in the proinflammatory response [582]. TNF- $\alpha$ also plays a central role in the pathogenesis of IBD and anti-TNF- $\alpha$ agents are used in the clinic to treat the disease [583]. TNF- $\alpha$ was also shown to increase intestinal epithelial permeability [584]. Young microbiota had a different effect and increased expression of lambda immunoglobulin light chain genes both in conventional and germ-free recipient mice. B cells express only one class of light chain, lambda $(\lambda)$ or kappa $(\kappa)$. It has been observed previously that the gut microbiota can influence the ratio of these two light chains. Microbial colonization of germ-free mice was shown to increase the ratio of $\lg \lambda^{+}$to $\lg \kappa^{+} B$ cells in the lamina 
propria [585]. Since increased $\lg \lambda$ usage by B cells is considered a marker for B cell receptor editing [586-588], these results might suggest that the young microbiota promote a more diverse B-cell repertoire. Another possibility is that the young microbiota contains more antigens that are recognized by $B$ cell clones that express $\lg \lambda$.

The increased level of differentiated $\mathrm{CD}^{+} \mathrm{T}$ cells in the spleen, the elevated inflammation in the ileum, and the prediction of LPS as an upstream regulator in the presence of aged microbiota led us to hypothesize that more bacterial components had translocated into the circulation in animals containing old microbiota. Indeed we observed that serum of germ-free recipients of old microbiota had an increased ability to activate TLR2 and TLR4. Similar mechanisms seem to contribute to other disorders such as type 2 diabetes and metabolic syndrome. High fat diet was shown to alter gut microbiota composition, which increased the permeability of the small intestine [573]. The increased permeability allowed bacterial components to reach distal sites, which induced low-grade inflammation and subsequent insulin resistance [572]. Importantly, the mucin-degrading bacterium Akkermansia muciniphila was shown to reverse these metabolic disorders by strengthening the intestinal barrier [589]. In our study old conventional mice had lower abundance of Akkermansia, which has also been reported previously both in humans and mice $[590,591]$. Akkermansia was also less abundant after transfer of old microbiota to germ-free mice at early time points. Therefore, it is tempting to speculate that the absence of Akkermansia in recipients of old microbiota might be associated with translocation of inflammatory bacterial components into the circulation.

As mentioned previously, certain members of the gut microbiota modulate the immune system [592]. However, components of the immune system such as $\operatorname{IgA}$ antibodies also shape gut microbiota composition [593-595]. Therefore, we investigated whether after transfer to germ-free mice the aged microbiota remained similar in composition to the donor or would quickly adapt to the young host. One week after transfer, the composition of old and young microbiota was clearly different, but after 4 weeks the difference was less pronounced, and both the microbiota from the old and young mice were more similar to the microbiota of the young mice. This suggests that the aged microbiota had partially adapted to the young host. As described for aged humans [549, 550], old conventional mice had a lower Firmicutes/Bacteroidetes ratio. However, this trait was not transferable to germ-free mice. Four weeks after transfer germ-free recipients of old microbiota had more TM7 bacteria and Proteobacteria. The difference in Proteobacteria was at least partially due to a significant lower abundance of Desulfovibrio after transfer of old microbiota. Interestingly, Desulfovibrio and TM7 bacteria have recently been associated with a compromised intestinal barrier due to an altered mucus structure that was more penetrable by bacteria leading to increased intestinal immune infiltration [596]. Further indications that TM7 phyla and Proteobacteria such as Desul- 
fovibrio can contribute to intestinal inflammation comes from observations that these bacteria are associated with the pathogenesis of IBD [597-599].

Together our results suggest that aged microbiota contributes to the chronic low grade inflammatory state observed during aging. Therefore, strategies to modify gut microbiota composition of the elderly with for example probiotics or prebiotics [600] might contribute to reduction of inflammation and thereby promote healthy aging. 


\title{
Chapter 9
}

\section{General discussion}

\author{
A.A. van Beek
}


Chapter 9 
The immune system is dysregulated with aging. The aging immune system is predominantly affected in two ways: 1 ) it becomes less capable to mount sufficient responses and to defend the body against pathogens, and 2) it becomes vulnerable for (auto)inflammatory conditions and inflammaging. These two aspects, which can be summarized in the term 'immunosenescence', contribute to the increased prevalence of cancer and infectious diseases in elderly (>65-year-old) [12-14]. More than half of the elderly have multiple age-related diseases [49]. As a result, the quality of life of most elderly is reduced. Preventing or reverting age-related defects in the immune system is therefore crucial to promote healthy aging.

Many detailed studies over the last decade, both in man and in mouse, have substantially increased our knowledge on the aging immune system. Clearly, in all cell types of the immune systems effects of aging have been identified, as described in the introduction of this thesis (Chapter 1). We explored the effects of aging on various components of the immune system and the gut (Figure 1), in particular in relation to the concomitant changes in the microbiota composition that are characteristic for aging. Our main findings are that basophils interact with dendritic cells (DC; chapter 2), and that basophils change with age in frequency and phenotype (Chapter 3). In Chapter 4, we reviewed that macrophages show decreased Toll-like receptor (TLR) signaling and increased IL-10 and prostaglandin (PG)E-2 production. We report that the mucus layer in the gut declines with age, which is prevented by treatment with Lactobacillus plantarum WCFS1 (Chapter 6 ). In line with literature, we describe that B cell and T cell precursors decline with age, and that dietary tryptophan restriction arrests $B$ cell development (Chapter 7). Finally, we provide evidence that microbiota from aged mice induce expression of TNF in the gut of young germfree mice, similar as in aging (Chapter 8).

\section{The aging immune system}

We studied the effects of aging on several types of immune cells: basophils, DC, macrophages, B cells, T cells, and innate lymphoid cells (ILC).

\section{Basophils: functional defects with aging?}

Basophils have been increasingly studied since four studies were published on their role in the initiation of Th2 immune responses [201, 222, 252, 253]. As discussed in chapter 2, these data were (in part) debated due to the simultaneous depletion of basophils and FceRla ${ }^{+}$DC [254], and are thus far not reproduced in humans. In addition to reviewing current literature on basophils, we added preliminary data on the interaction between basophils and DC in vitro. Basophils have the capacity in vitro to change the phenotype of and cytokine production by DC, and vice versa. Although we have 
Chapter 9

not investigated how basophils and DC interact with each other mechanistically, both cell-cell interactions and secretory mediators may play a role. We concluded that it is of interest to explore the effect of aging on basophils.

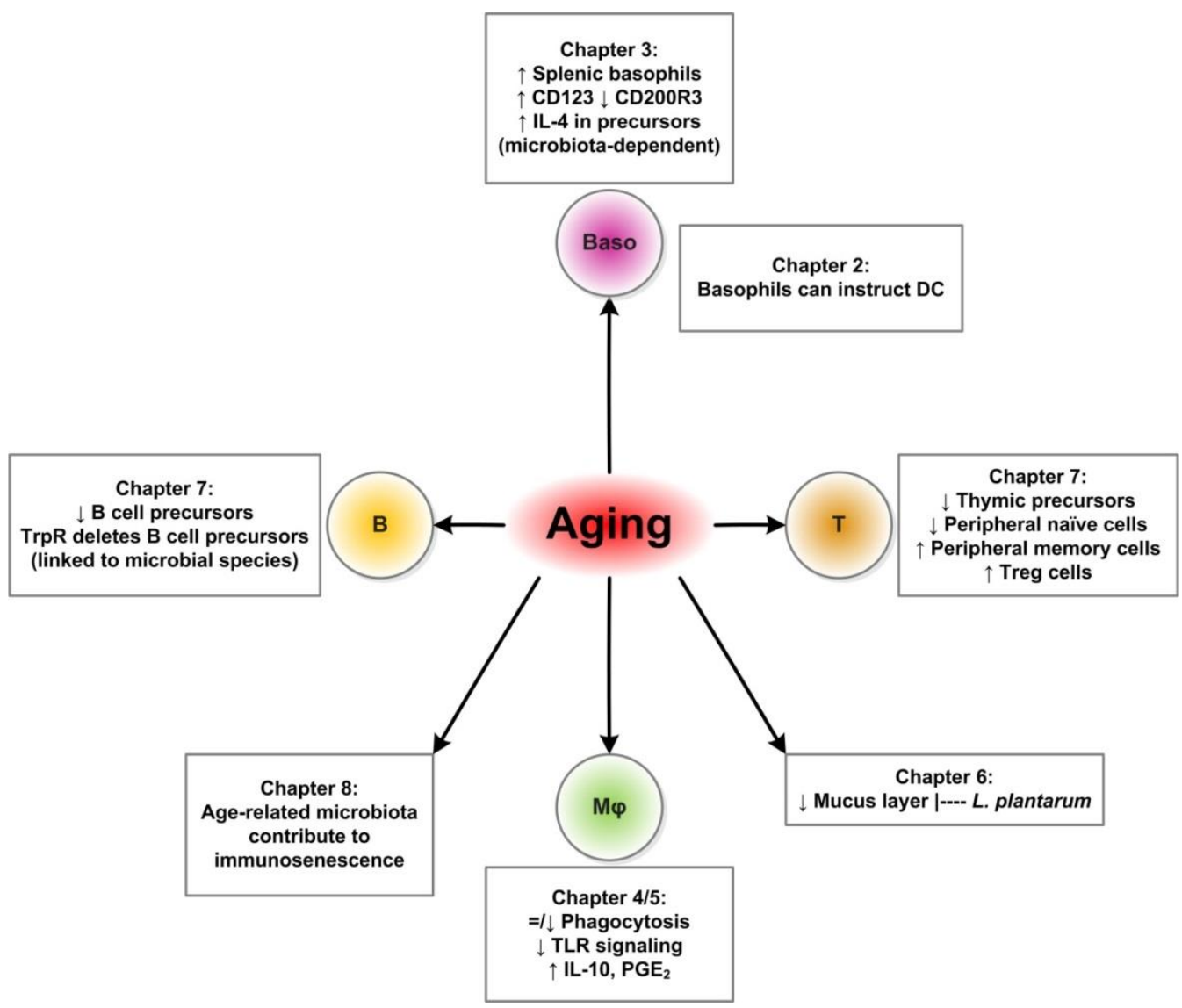

Figure 1. Overview of main findings in this thesis. $B=B$-lineage cells; $B a s o=$ basophils; $D C=$ dendritic cell; $M \varphi=$ macrophage; $P G=$ prostaglandin; $T=T$-linage cells; $T r p R=$ dietary tryptophan restriction.

In chapter 3, we explored basophils in young and aged mice. We report that basophil frequencies increased in the spleens of aged mice, while no changes in basophil frequency were found in the bone marrow (BM). In humans, basophils steadily decline in the blood with age [73]. There are, however, no data on basophils frequencies in aging human spleen or BM, or mouse blood, hampering the comparison between this human study and our data. Moreover, there are conflicting data whether Th2 responses in general decline with age [601]. Busse et al (2007) studied ovalbumin (OVA)specific responses in the lungs of mice of different ages. They, however, mainly focused on eosinophils, and data on basophils are lacking [602]. Summarizing, we report that basophils interact with DC, and that basophil frequencies and phenotype change with age. 
Future directions: Whether our findings translate into a basophil-dependent defect in Th2 responses in vivo with aging remains to be elucidated. Allergic airway inflammation in young and aged mice could be compared to learn whether the age-related changes in basophils determine outcome of Th2-related inflammation in vivo.

\section{$D C$ and aging}

DC are important immune cells in the connection between the innate and the adaptive immune responses. In elderly, the total numbers of DC seem to be unaffected [603, 604]. We also have studied DC in aging mouse BM, spleen, and mesenteric lymph nodes (MLN), and after bacterial supplementations, but we have not found significant differences in distribution (data not shown). Several human studies, however, show a reduction in plasmacytoid $\mathrm{DC}(\mathrm{pDC})$ and no change in myeloid $\mathrm{DC}$ in peripheral blood [605-607]. A decrease in the number of Langerhans cells, and an altered morphology were observed in elderly [604]. In aged mice, an altered distribution of DC subsets was found as well [608].

Phagocytosis, pinocytosis, migration and chemotaxis were impaired in DC from aged donors, leading to a decreased ability to present antigens [63, 110,603, 609]. As a result, aged $D C$ have a reduced ability to stimulate $T$ cell responses, and are less able to control tumor growth $[110,609,610]$. Although a number of conflicting studies exist, the vast majority of studies point to a general decline in DC function with age [611]. These age-related changes may also provide an explanation for the disrupted interaction of DC with $B$ cells and a decreased priming of $T$ cells in lymph nodes [63]. Comparable and consistent results on DC are hampered by the use of various protocols, which differ in source (blood, BM, cord blood) and culture conditions. In general, these data indicate that DC phenotype and functions are affected with aging.

\section{Macrophages and aging}

Macrophages are critical regulators of processes aimed at maintaining homeostasis, such as inflammatory and immune responses [612], and metabolism [613]. Chapter 4 includes a summary of age-related alterations in macrophage numbers, phenotype, and functions, and concludes with potential (dietary) interventions to rejuvenate aged macrophages. In our studies, we included analyses of peritoneal macrophages $\left(\mathrm{CD} 11 \mathrm{~b}^{\mathrm{hi}} \mathrm{F} 4 / 80^{\mathrm{hi}}\right)$. Although we found no change in macrophage numbers or phenotype in the peritoneum of aged WT or $E r C c 1^{-1 \Delta 7}$ mice, as compared with young WT or $E r c c 1^{-/ \Delta 7}$ mice (data not shown), it is nevertheless conceivable that aging affects macrophage function. Because macrophages are central in immunity, we review in chapter 4 changes in polarization, epigenetic and metabolic processes, and autophagy in aging macrophages as potential driving factors for inflammaging. It is now accepted that inflammatory responses are tightly regulated and integrated by epigenetic processes, 
Chapter 9

which influence the transcription of genes by DNA methylation or histone-tail modification. General DNA hypomethylation as well as consistent histone modifications are hallmarks of aging [614]. For example, SIRT1 (protein deacetylase) activity prevents cognitive decline and neurodegeneration in aging via histone modification [615]. Epigenetic processes are sensitive to nutritional components, such as omega-3 fatty acids [616] and microbe-derived butyrate [617]. Because the epigenetic landscape changes with age $[618,619]$, modifying the microbial composition (by tryptophan restriction or by supplementation of bacterial strains) might have consequences for the epigenetic landscape in aging individuals. Because the epigenetic landscape is sensitive to environmental factors, its analysis should be included in future studies regarding nutritional interventions. In sum, macrophage distribution, phenotype, and functions change with age. Their prime role in inflammation makes these changes probably important in the inflammaging process.

\section{Lymphocytes and aging}

Primary lymphoid organs thymus and bone marrow involute with age [55, 488]. These and other age-related effects are more broadly discussed in chapter 1. In line with previous studies, we report that the precursors of B cells in the BM and precursors of $T$ cells in the thymus decrease in numbers with age (chapter 7 ). We also found increased memory $T$ cell frequencies (at the expense of naïve $T$ cells) and increased Treg frequencies, which is supported by previously reported findings [525, 526]. We also studied whether ILC2 were affected with age. When comparing the frequencies of Lin ${ }^{-G A T A 3}{ }^{+} \mathrm{CD} 127^{+}$ILC2 in spleens of 16 -week-old $\mathrm{ErCC1}^{+/+}$mice with 16-week-old ErCC1 $^{-/ \Delta 7}$ mice, we observed no significant change ( $0.06 \%$ vs. $0.08 \%$; data not shown). In summary, we confirmed that lymphocyte distribution changes with age.

Future directions: Data on ILC need to be confirmed in comparing young and aged wild-type mice, and should take into account ILC1 and ILC3 subsets as well.

Together, these data show that aging has profound impact on the immune system. An important question is how to prevent or maybe even revert these age-related changes. We have studied the role of probiotics, dietary tryptophan restriction, and microbial transfers (chapter 5/6, 7, and 8, respectively). In all these studies, microbiota play a significant role, either directly (as a bacterial strain or a complete microbiota) or indirectly (via tryptophan restriction). 


\section{In vivo aging conditions challenge the suitability of in vitro cytokine ratios to predict efficacy of probiotics}

Selection of bacterial strains in vitro

We used splenocytes and bone marrow-derived macrophages to evaluate probiotic properties of bacterial strains in vitro (chapter 5). We showed that splenocytes and macrophages of aged mice have an altered response to LPS and bacterial strains as compared with those of young mice. IL-10/IL-12 ratios have been widely used to assess probiotic activity in vitro. As discussed in chapter 5, IL-10/TNF ratios were similar to IL-10/IL-12 ratios in a previous study. Based on differential IL-10/TNF production ratios of splenocytes in young mice, we applied three bacterial strains in vivo. Lactobacillus plantarum WCFS1 was classified as pro-inflammatory (lowest IL-10/TNF), L. casei BL23 as regulatory (intermediate IL-10/TNF), and Bifidobacterium breve DSM20213 was classified as anti-inflammatory (highest IL-10/TNF).

The predictive value of IL-10/TNF ratios in aging

We found that the in vitro classification of L. plantarum, L. casei, and B. breve did not match with our findings on application in vivo to $\operatorname{Ercc} 1^{-/ \Delta 7}$ mice (chapter 6$)$. In brief, we found that $L$. casei induced Tregs, but also raised several inflammatory markers. $L$. plantarum induced Tregs, without showing an increase in pro-inflammatory cells or cytokines. B. breve did not show anti- or pro-inflammatory activity, but induced damage in the ileum (Van Beek et al, unpublished findings). These findings challenge the use of IL-10/IL-12 (or IL-10/TNF) ratios to screen for candidate probiotics with antiinflammatory capacities [472] in the aging host. It may even be argued that strains scoring high IL-10/IL-12 ratios - aimed to combat inflammaging - are not beneficial in the aging context, because IL-12 induction is impaired in aging while IL-10 production is generally elevated [475]. It has been reported that cytokine profiles of TLRstimulated whole-blood from elderly are altered compared with young adults, favoring IL-6, TNF, and IL-10 production, at the expense of e.g. IL-12, and IL-1 $\beta$ [475]. Extrapolating these data to the colon, and combined with this study, it might mean that high IL-10 induction indeed is not beneficial in the aging intestine, but that IL-1 $\beta$ induction (as observed after L. plantarum supplementation) might be beneficial.

Inclusion of age in the definition of probiotics

In young wild-type $\left(\mathrm{Ercc1}^{+/+}\right)$mice, no major effects of supplementation with any of the three selected bacterial strains were found on intestinal barrier and immunity in Peyer's patches (PP), MLN, or spleen (chapter 6). However, after supplementing accelerated aging Ercc1 ${ }^{-1 \Delta 7}$ mice with L. plantarum, we found that this bacterial strain prevented the age-related decline in mucus barrier. At the same time, its supplementation 
Chapter 9

upregulated IL-1 $\beta$, IL-4, IFN- $\gamma$, and TNF in colonic tissue, and induced higher Treg frequencies in MLN. Supplementation with L. casei or B. breve in $\operatorname{Ercc}^{-1 / 47}$ mice resulted in raised inflammatory parameters in the spleen (a.o. neutrophils and Ly6C ${ }^{\text {hi }}$ monocytes) or exacerbation of the age-related decline in the intestinal barrier, respectively.

The comparison of our findings in $E r c c 1^{-/ \Delta 7}$ mice with those in $E r C C 1^{+/+}$mice underlines that it might be important to pay careful attention to the age of individuals that receive bacterial supplementation. The aged gut of $\operatorname{Ercc}^{-1 / \Delta 7}$ mice responded in a different way to candidate probiotic strains than the young gut of $\mathrm{Ercc}^{+/+}$mice. The aging condition of the gut barrier and immune system reveals the beneficial effect of $L$. plantarum versus the detrimental effects of $L$. casei and $B$. breve, while these are not observed in young wild-type mice. To the best of our knowledge, we are the first to compare directly the effect of candidate probiotic strains in young and aged mice. Our findings might have significant implications for studies in humans. Probiotics have been tested in aged subjects, with many examples of beneficial strains (see chapter 1 and chapter 7), but no direct comparison between young and aged individuals has been performed.

Probiotics are defined by the WHO/FAO as follows: "live microorganisms which when administered in adequate amounts confer a health benefit on the host" [160]. A recent review indicates 9 selection criteria for probiotics: 1) health benefit on the host; 2) viability upon intake; 3) survival during passage; 4) adherence to the gut epithelium; 5) antagonism against pathogens; 6) stabilization of microbiota composition; 7) human origin; 8) stability to bile, acid, enzyme, oxygen; and 9) safety (includes being nonpathogenic, non-toxic, non-allergic, non-mutagenic) [620]. The notion that the condition of the individual plays an important role is confirmed in a different setting. In a study with patients suffering from severe pancreatitis, enteral supplementation of the probiotic mixture Ecologic 641 (Bifidobacterium bifidum, Bifidobacterium infantis, Lactobacillus acidophilus, Lactobacillus casei, L. salivarius, Lactococcus lactis) increased mortality risk [621]. The choice for Ecologic 641 was based on in vitro studies showing that it induced high levels of IL-10 and low levels of TNF, IL-2, and IL-6. In addition, the probiotic mixture showed strong antimicrobial properties [622, 623]. In the WHO/FAO definition, and in these 9 criteria, no criterion addresses the age or other immune parameters of the host. Our studies, both in vitro (chapter 5) and in vivo (chapter 6), show that caution is needed when translating effects by probiotics from young subjects to aged subjects.

Future directions: Based on our study, we recommend that the definition of probiotics should at least include age (and related health status) of the host. 


\section{Microbial colonization and gut immunity}

The role of microbiota in the mucosal immune system has been recognized for many decades. Microbial colonization stimulates activation and terminal differentiation of $B$ cells in the gut [624], resulting in class switching in B cells with increased numbers of intestinal IgA plasma cells [625]. The mucosal antibody repertoire develops in response to the intestinal microbiota [626]. In addition, microbial colonization is important for the induction of Tregs and for maintaining the balance between Th1, Th2, Th17 and Treg cells [577, 627, 628]. The development of the immune system in germfree mice, devoid of microbiota, can be stimulated by microbial components present in the diet, such as LPS [629]. LPS has been shown to enhance directly the suppressor function of Treg via TLR4 [630]. Thereby, LPS can act as a suppressor of T cell-dependent antibody responses [631]. In the absence of microbiota, there are smaller and fewer PP and isolated lymphoid follicles (ILF), a thinner lamina propria, and developmental defects in epithelial cells, $T$ cells, and B cells [632].

A recent study showed that elevated MCP-1/CCL2 serum levels in aging associated with the gut microbiome composition of mice [633], indicating the potential effect of microbiota on the aging immune system. In our studies, many examples of interaction between the immune system and microbiota were identified. Chapter 2 and 5 describe the in vitro interaction of bacterial strains with basophils and/or DC, and splenocytes or macrophages are described. In chapter 6, 7, and 8, we describe the in vivo effects of bacterial strains, microbial changes after dietary tryptophan restriction, and microbiota transfers, respectively. In chapter 8 , we showed that microbiota from aged mice enhanced Th1 cells and Tregs in the spleen. This indicates that gut microbiota also induce immune cell differentiation in non-mucosal immune organs. Although the nonredundant role of microbiota in mucosal immunity has been firmly established, a link between gut microbiota and development of immune cells in the BM has only been discovered more recently.

\section{Gut-BM axis and diet: consequences for basophil and B cell development} Neutrophil and basophil development in the BM are under control of microbiota A relation between gut microbiota and immune cell abundance, phenotype, and function has been established for hematopoietic stem cells (HSC), neutrophils, and basophils. Repeated intraperitoneal exposure to LPS results in a myeloid bias of HSC and gain or loss of specific HSC populations [512]. Gut microbiota from mice fed high-fat diet (compared with mice fed normal diet) changed the stem cell niche in the BM, enhancing the myeloid cell development at the expense of lymphoid cell development [279]. Not only HSC are affected by gut microbiota, but also neutrophils and basophils. Intestinal translocation of peptidoglycan (PGN) from gut microbes primes neutrophils 
Chapter 9

in the BM in a NOD1-dependent way to kill Salmonella pneumoniae and Staphylococcus aureus. Lower levels of PGN in serum correlated with hampered neutrophil function against these pathogens [280]. In the airways, Staphylococcus aureus colonization resulted in altered polarization of macrophages and protection against influenzamediated inflammation through TLR2-dependent signaling [634]. Gut microbes caused downregulation of CD123 (IL-3Ra) expression on basophil precursors, thereby limiting their responsiveness to IL-3 [281].

In chapter 3 , we describe an optimized method to generate basophils from precursors in the BM in an IL-3-dependent culture, and to isolate these BM-derived basophils. We applied this culture method to assess the differentiation of basophil precursors and the function of basophils. We showed that with age the proportion of $\mathrm{IL}-4^{+}$basophils increases upon various stimuli. We found that microbiota of aged mice (versus microbiota of young mice) also induced higher proportions of IL-4+ basophils after in vitro differentiation of precursors. We thus confirmed that microbiota change basophil precursors in the BM, leading to an increased proportion of basophils that produce IL-4 (Figure 2).

Future directions: It remains to be elucidated what these findings mean in allergies or helminth infections, and how these findings translate to humans. In addition, it remains to be determined what (other) molecular mechanisms are involved in the interaction between microbiota and stem cell niches in the BM.

\section{Does $B$ cell development depend on gut microbiota?}

In contrast to above-mentioned evidence that microbiota-derived signals prime $B$ and T cells in the gut, no such evidence has been described for their development in the BM or thymus. Our findings in chapter 6 that specific precursor stages (i.e. small resting pre-B cells) are significantly decreased after $L$. casei supplementation, and to a lesser extent after $L$. plantarum supplementation, suggest a role for microbial ligands in B cell development. In chapter 7, we showed a correlation between Akkermansia muciniphila abundance in the colon and numbers of three stages of $B$ cell precursors in the BM.

Some evidence that suggests a role of microbial ligands in B cell development has been previously reported. In vitro, B cell precursors mature in response to LPS or lipid A (TLR4 ligands). This maturation is inhibited by Pam3Cys (TLR2 ligand). In addition, lipid A and Pam3Cys impairs IL-7-dependent proliferation [635]. B cell precursors express TLR9, and are, even after IL-7 withdrawal, protected against apoptosis by TLR9 ligand $\mathrm{CpG}$ [636]. In addition, $\mathrm{CpG}$ injection stimulates class switch recombination of $B$ cell precursors in vivo [637]. LPS injection increases mature $B$ cell frequencies at the expense of pre-B cells in vivo [638]. This finding is corroborated by similar findings upon LPS injection of TLR4-mutant mice, receiving B cell precursors from TLR4-intact 


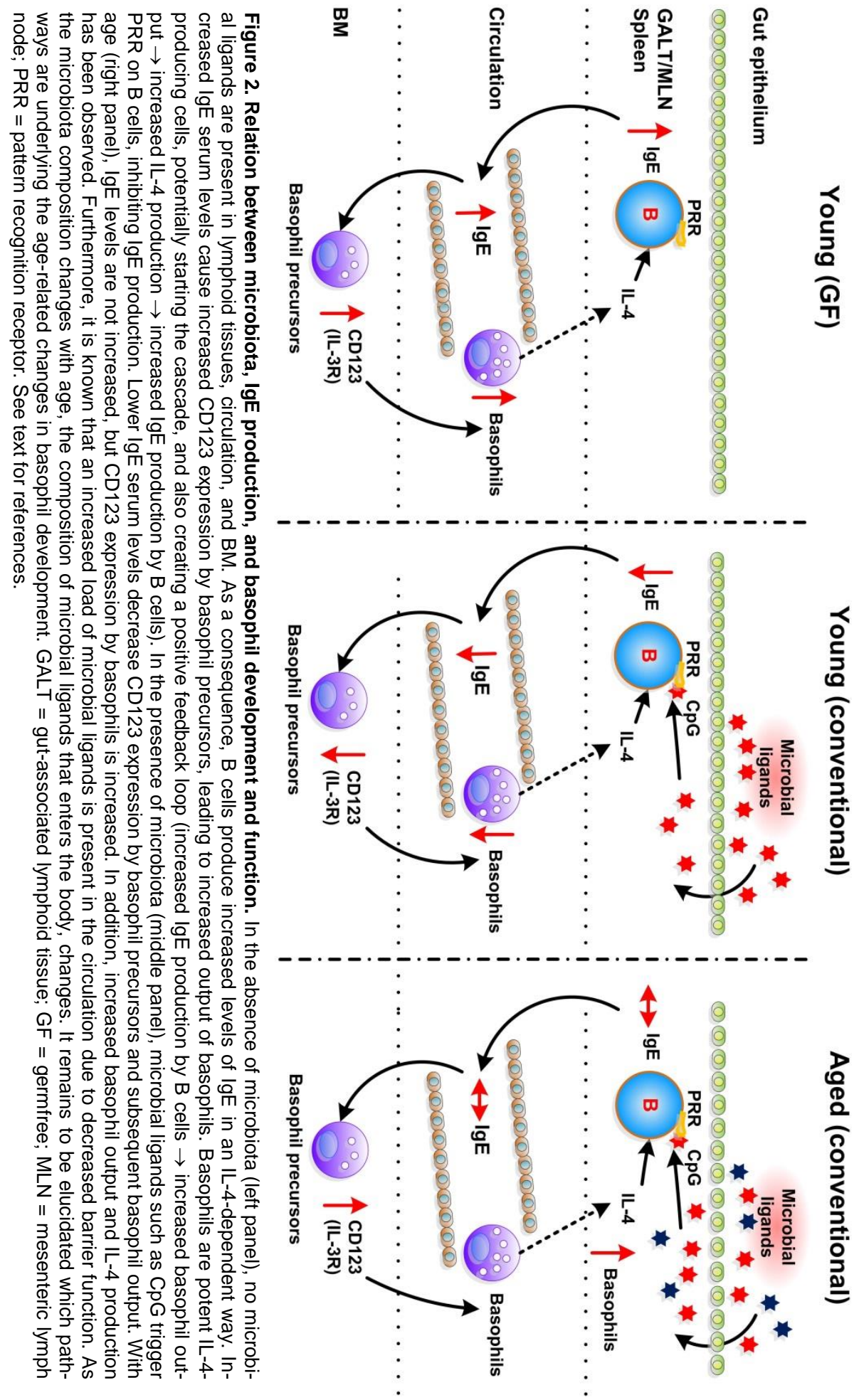


mice [639]. Together, these data suggest that B cell development in the BM is influenced by (components of) gut microbiota.

\section{$B$ cell development in germfree mice, and effect of microbiota introduction}

Germfree mice represent a valuable model for the study of the role of microbiota in immunity. To our best knowledge, no data are available on lymphopoiesis in the BM of germfree mice and germfree mice that receive microbiota. By analyzing the $B$-lineage in the $\mathrm{BM}$, we found more evidence that microbiota control $\mathrm{B}$ cell development. In the absence of gut microbiota, B-lineage cells were more abundant in the BM (Figure 3A; Van Beek et al, unpublished findings). Introduction of microbiota in germfree mice resulted in B-lineage cell frequencies as observed in conventional mice (Figure 3A). Cells in all stages of $B$ cell development, except the pro-B cell stage, were significantly increased in the absence of microbiota, and all increases were reverted upon introduction of microbiota. Increased abundance of B-lineage cells occurred at the expense of

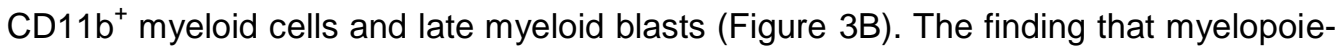
sis is decreased in the absence of microbiota, is in line with a previous study [640]. This might be related to the finding that germfree mice have less adipose tissue than conventional mice, accompanied by lower adiponectin expression in ileum and liver [641], and lower serum levels of leptin [642]. Leptin enhances myeloid cell development [643], but is not required in normal B cell development [644]. Adiponectin blocks $B$ lymphopoiesis [530]. Interestingly, leptin and IL-1 $\beta$ are identified by in silico analysis as activated upstream regulators in $L$. plantarum-treated $\operatorname{Ercc1}^{-/ \Delta 7}$ mice (chapter 6), and we speculate that these factors might explain the slight decrease in BM B cells after $L$. plantarum supplementation. Taken together, germfree mice have decreased expression of factors that stimulate myelopoiesis (leptin [642]) or block lymphopoiesis (adiponectin [641]), resulting in increased B cell development at the expense of myeloid development (Figure 3). Interestingly, Khosravi et al (2014) demonstrated that microbiota are necessary for the defense against Listeria monocytogenes. It is unclear which microbe-associated molecular patterns (MAMP) instruct myelopoiesis, as the authors used heat-killed E. coli [640].

Future directions: To establish a causal relationship between factors like leptin and adiponectin, microbiota, and lymphopoiesis, more research is warranted. For instance, the effect of leptin or adiponectin injections on B cell development should be evaluated in germfree and conventional mice. Correlating the lymphopoiesis and microbiota composition in leptin or adiponectin knockout models could also give insight into the role of these factors in B cell development and microbiota composition. Moreover, analysis of B cell development should be performed after oral treatment of germfree mice with MAMP. 
Thus, several pieces of indirect evidence and correlations with microbiota, whether specific (Akkermansia, chapter 7 ) or in general (Figure 3), strongly suggest that B cell development is under control of gut microbiota. Direct evidence for a causative role of microbiota in B cell development, however, is lacking.
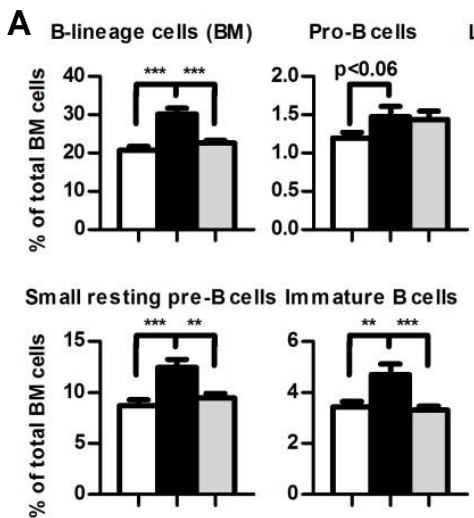

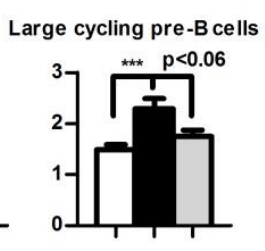

Mature B cells

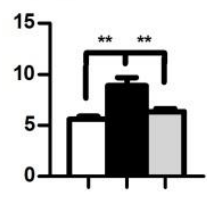

B
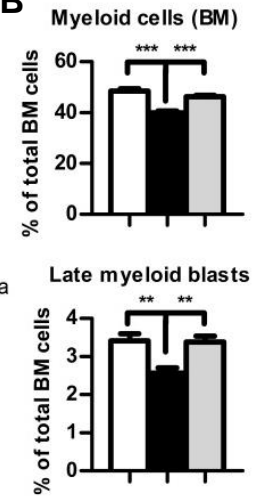

Figure 3. Effects of absence and introduction of gut microbiota on B cell development and myeloid cell development. A) Mean frequencies of (stages of) B-lineage cells in the BM. B-lineage cells were defined as $C D 19^{+} C D 45 R^{+}$. B-lineage cells divided into slgk/ $\lambda^{-} B$ cell precursors (and subsequently into clgM ${ }^{-}$ CD2 pro-B cells, clgM ${ }^{+} \mathrm{CD2}$ large cycling pre-B cells, and clgM $^{+} \mathrm{CD2}^{+}$small resting pre-B cells) and slgk $/ \lambda^{+} \mathrm{clgM}{ }^{+} \lg \mathrm{D}^{-}$immature and slgk $/ \lambda^{+} \mathrm{clgM}{ }^{+} \lg \mathrm{D}^{+}$mature $\mathrm{B}$ cells. B) Mean frequencies of $\mathrm{CD} 11 \mathrm{~b}^{+}$myeloid cells and Ly6C ${ }^{+} \mathrm{CD} 31^{+}$late myeloid blasts in the BM. Data represent the mean + SEM of $n=9-20$ mice, analyzed with unpaired Student's $t$ tests, or Mann-Whitney tests if not passing D'Agostino \& Pearson omnibus normality test. All mice were about 4-month-old. $\mathrm{BM}=$ bone marrow; CONV = conventional; GF = germfree; $\mathrm{GF}+$ microbiota $=$ germfree mice inoculated with gut microbiota from CONV mice (analysis took place 4 weeks after the inoculation). Van Beek et al, unpublished findings.

\section{The influence of aging on B cell development}

B cell development decreases with aging [92, 100], which is confirmed by our studies (chapter 7). The ability to respond to IL-7 is impaired in pro-B cells from aged mice [645]. With aging, stromal cells produce less IL-7 [646]. Stromal cells in the BM produce several other factors that enhance $B$ cell development, including CXCL12, Flt3 ligand (FIt3L), stem cell factor (SCF), and receptor activator of NF-KB ligand (RANKL) $[647,648]$.

Adipocytes in the BM produce adiponectin, which blocks B cell development [530]. B cell development is inhibited by IL-1 $\alpha$, IL-1 $\beta$, and another (yet unidentified) adipocytederived mediator $[649,650]$. Of note, hematopoietic tissue in the BM is increasingly replaced with adipose tissue with aging $[62,651]$, and adiponectin levels in serum are increased with aging [652]. IL-1 $\beta$ levels are also elevated in elderly [71, 653]. Combined with intrinsic defects in B cell precursors, a decrease in IL-7 and an increase in inhibiting mediators with aging might explain the decreased $B$ cell development in the BM (Figure 4). Importantly, we showed in chapter 8 that microbial ligands are more 


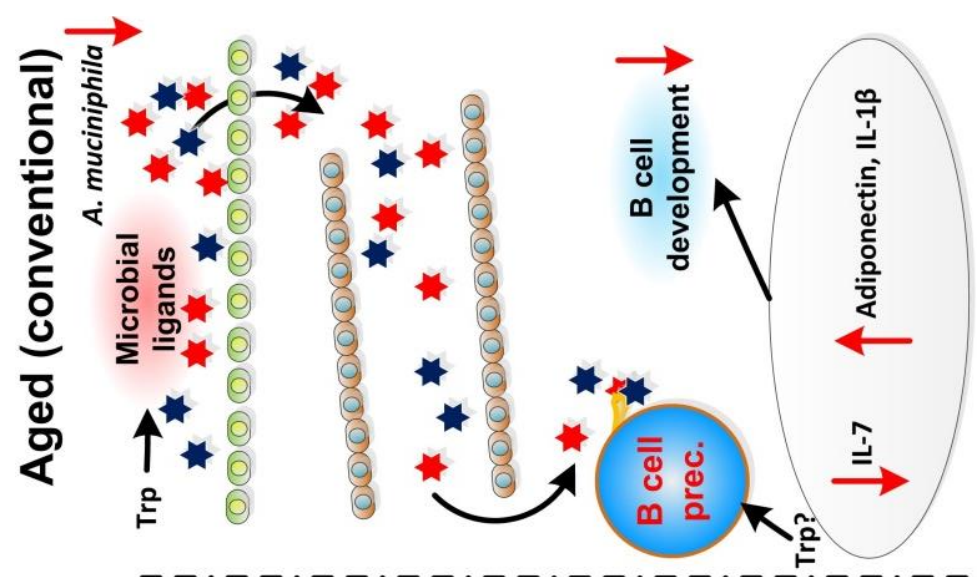

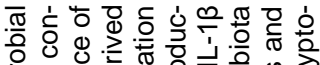
응 웡

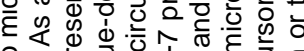

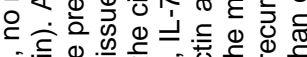
बิे 宛

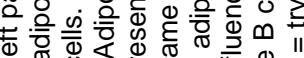

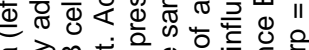

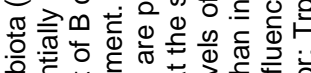

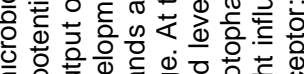

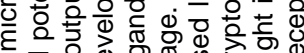

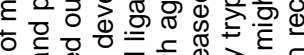

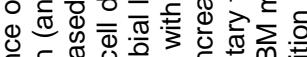
呅 品

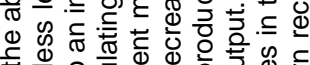

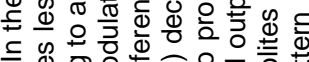

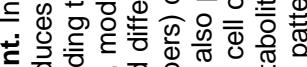

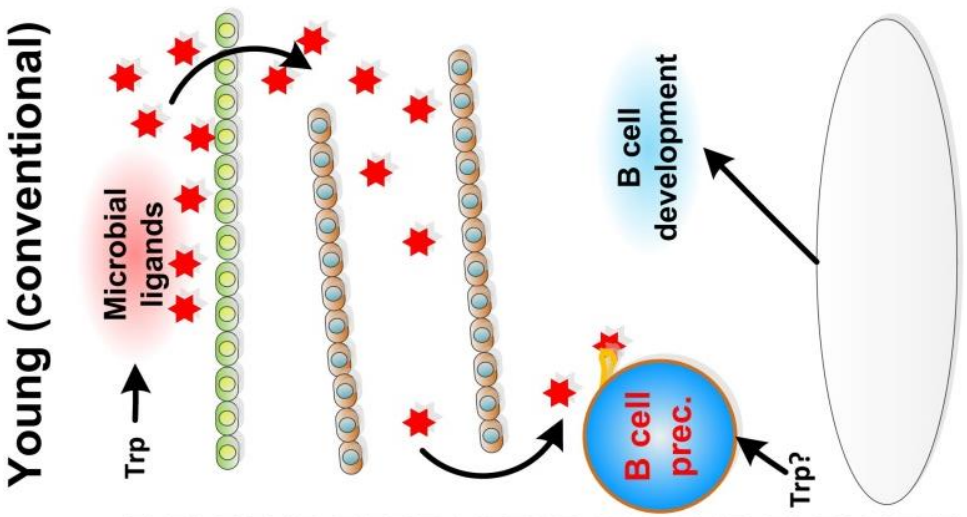
屯

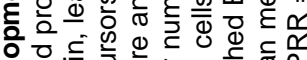

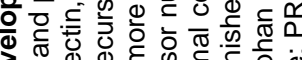
屯ั

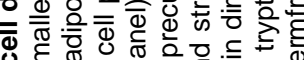

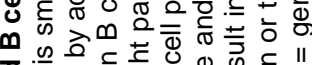

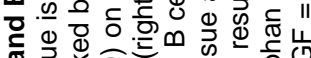

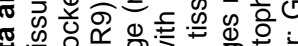
픙.

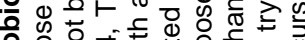

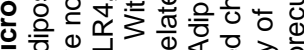

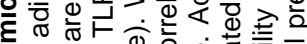

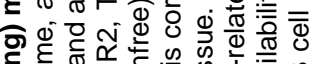
등 đ v vi 屯

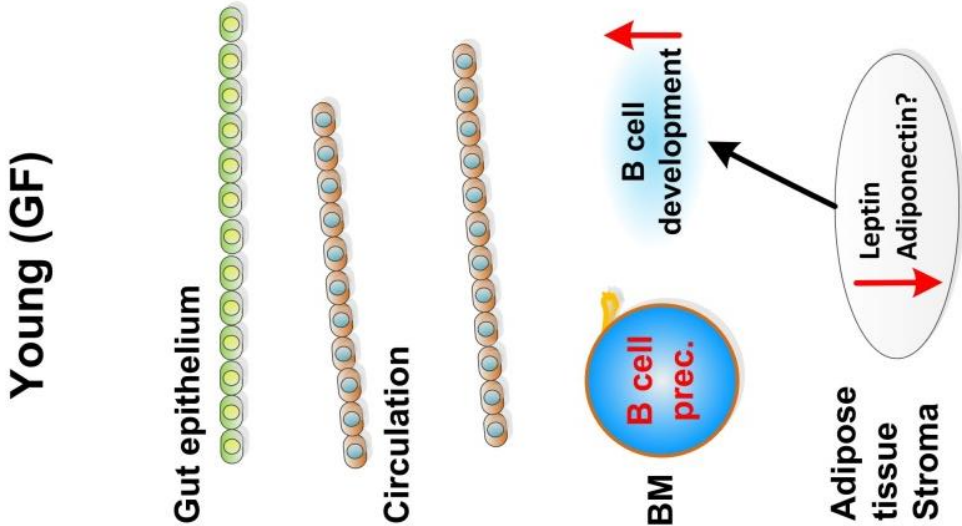

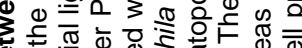

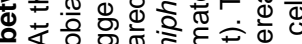

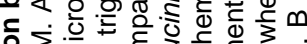

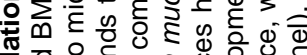

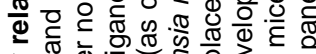

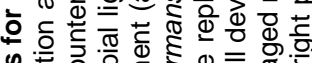
क⿻ क.

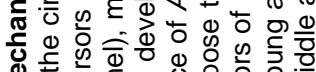

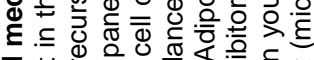

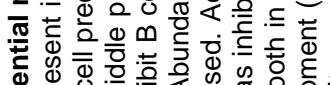

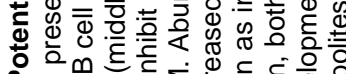

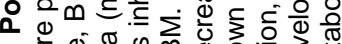

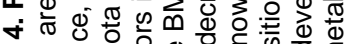

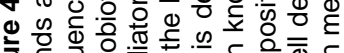

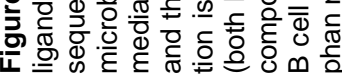


abundant in the circulation after transferring aged microbiota to germfree mice (compared with transferring young microbiota). This demonstrates that microbial ligands are present in the circulation, and can thus affect B cell development in the BM (Figure 4).

An essential role of tryptophan (metabolites) in B cell (but not myeloid) development Studies into the role of tryptophan in immunity have predominantly focused on the role of IDO-mediated scavenging of Trp by DC, induction of Tregs, and subsequent immunosuppression [178]. In our study, however, we found that B cells are particularly targeted by dietary tryptophan restriction (chapter 7). Strikingly, B cell development was arrested, whereas myeloid cell development in the BM was unaltered (both in cell numbers and proliferation). Although a general increase of memory $T$ cells at the expense of naïve $T$ cells was observed, $T$ cell frequencies were increased in the periphery. At the same time, all types of B cells were reduced in frequency, except marginal zone (MZ) B cells. These data show that dietary tryptophan restriction exerts a previously unknown effect on B cell development.

Future directions: Further studies are warranted to investigate the role of pattern recognition receptors (PRR) on $B$ cell precursors and dietary tryptophan restriction, as we report that abundance of Akkermansia muciniphila and Alistipes are correlated with $B$ cell precursor numbers (chapter 7). This might be done by assessing the effect of dietary tryptophan restriction in mice that are deficient in MyD88, which is central in the signaling of many PRR [654]. To evaluate the role of (changing) microbiota composition, it would be worthwhile to apply dietary tryptophan restriction in germfree mice or antibiotic-treated mice, and compare the effect to tryptophan-restricted conventional mice. It is also possible that the decreased availability of aryl hydrocarbon receptor (AHR) ligands, like tryptophan metabolites, causes alterations in B cell development. Indeed, AHR signaling controls innate and adaptive immunity through regulation of tryptophan metabolism [655]. $\mathrm{AHR}^{-/-}$mice had increased $\mathrm{B}$ cell precursor frequencies (compared with $\mathrm{AHR}^{+/+}$). Supplying $\mathrm{AHR}^{+/+}$mice with dioxin (AHR ligand) decreased $\mathrm{B}$ cell precursors, but had no effect on $B$ cell precursors in $A \mathrm{HR}^{-/-}$mice [656], indicating that $A H R$ and $A H R$ ligands play a role in $B$ cell development. The direct effect of tryptophan depletion on B cell development might be assessed in e.g. IL-7-driven BM cultures, with or without tryptophan depletion. To identify changes in aging, (accelerated) aged mice should be studied along the lines as described above. The first step to gather more direct evidence for a role of microbiota in B cell development could be to evaluate the effect of antibiotic treatments on B cell response and development - a similar approach as followed by Hill et al (2012) [281]. Then, comparing the B cell response to pathogens in germfree and antibiotic-treated mice with conventional mice would provide direct evidence for involvement of microbial ligands in B cell develop- 
Chapter 9

ment. Mechanistic studies should find out the role of PRR or other pathways involved in the role of microbial ligands in B cell development. These could include modified $L$. plantarum WCFS1 [186] or other modified bacterial strains.

\section{Similarity of tryptophan restriction and dietary restriction}

Tryptophan-restricted mice also showed a substantial decrease in body weight (chapter 7), which is very similar to mice receiving dietary restriction (DR) [657]. DR is widely studied for its extension of lifespan and health span [658]. In addition, DR has been shown to modulate the aging immune system [659] and microbiota composition [660]. Therefore, we tested the effect of $30 \%$ dietary restriction (DR) on BM composition of $\operatorname{Ercc1}^{-/ \Delta 7}$ mice (Figure $5 \mathrm{~A}$ ), and compared it with the effect of dietary tryptophan restriction (as described in chapter 7). When comparing the restriction diets, it is important to note that the mice receiving tryptophan restriction also received $10 \%$ DR (as well as their controls). The controls for the mice receiving 30\% DR received $0 \%$ DR. Also, the age of the mice at the start of restriction was different, as well as the feed. These differences may have caused a decrease or increase in BM populations, as is observed for lymphocytes, early blasts, myelomonocytic blasts, and erythroblasts (Figure 5B).

Lymphocytes in the BM (comprising mostly B cells) were decreased by about $50 \%$ by DR $(p<0.05)$, as well as early blasts (comprising mostly hematopoietic progenitors; $p<0.01$; Figure $5 B$ ). These latter populations were (not significantly) decreased by tryptophan restriction. In contrast, erythroblasts were increased upon $D R(p<0.05)$ and tended to increase upon tryptophan restriction $(p<0.10)$. Myelomonocytic blasts were significantly increased upon both restrictions $(p<0.05)$. No significant changes were observed in monocytes and granulocytes. We therefore conclude that DR particularly affects lymphopoiesis, in contrast to myelopoiesis.

As indicated above, direct comparisons are hampered by differences between the tryptophan restriction experiment and the DR experiment. Nevertheless, taking into account all measured cell populations in the BM, it is remarkable that tryptophan restriction not only resembles weight loss in $\mathrm{DR}$, but also resembles the major changes in BM composition. It is conceivable that the changes in B cell precursors as reported after dietary tryptophan restriction (chapter 7), are also occurring after DR. Indeed, two-week DR in young mice leads to an arrest in B cell development in the BM [661]. Additionally, based on the analysis of BM composition, it is likely that DR decreases the $\mathrm{B}$ cell precursor compartment in a similar fashion as tryptophan restriction.

As discussed above, dietary tryptophan restriction impacts on the gut microbiota. Changes in microbiota composition are correlated with B cell precursors (chapter 7). Strikingly, microbiota composition also changed after DR (Figure 5C), in line with previous reports [660]. There may, therefore, be a correlation between microbial species 
and specific stages in B cell development after DR (like after tryptophan restriction). Thus, tryptophan restriction resembles DR by weight loss, distribution of immune cells in the BM, and an altered gut microbial composition.

Future directions: To enable a correlation between microbial species and B cell precursors after DR, simultaneous analysis of B cell precursors and microbiota composition should be performed in mice receiving DR. We have tested 95\% tryptophan restriction. It would be interesting to find out whether a dose-response relation exists between dietary tryptophan restriction and e.g. B cell development. A study in young versus elderly people would give insight into applicability in humans. In fact, a shortterm dietary tryptophan restriction diet has been applied in humans before, resulting in enhanced prolactin response to intravenously injected tryptophan [662]. In addition, restriction of methionine has been shown to extend life span in rodents [173, 663, 664]. Interestingly, methionine restriction induces autophagy, and abolishing autophagy induction reverts lifespan extension in yeast [665]. Methionine restriction also prevents oxidative stress [173, 527, 528] and many age-related metabolic effects [529, $666,667]$. A combination of tryptophan/methionine restriction could therefore result in synergistic effects to extend life span. Also, it might be possible to apply less severe restriction of tryptophan and methionine, when combining these regimes. Yet, it has to be determined whether the immune system after tryptophan and/or methionine restriction is still capable of defending the body against pathogens.

Figure 5 (next page). Bone marrow and microbiota composition of old $\mathrm{Ercc1}^{-1 / \Delta 7}$ mice after dietary restriction or dietary tryptophan restriction. A) Flow cytometric analysis of BM composition. Live single cells were gated for Ly6C and CD31 to distinguish six immune cell populations. B) Mean frequencies of lymphocytes, early blasts, late myeloid blasts, erythroblasts, granulocytes, and monocytes. C) Redundancy analysis of microbiota composition of DR in $\mathrm{ErCC}^{+/+}$and $\mathrm{Erccl}^{-1 \Delta 7}$ mice, determined with Mouse Intestinal Tract chips. Ercc ${ }^{+/+}$DR control mice, Ercc1 ${ }^{+/+}$DR mice, Ercc1 ${ }^{-1 \Delta 7}$ DR control mice, and Ercc $1^{-/ \Delta 7}$ DR mice are depicted as black circles, blue squares, green diamonds, and brown X, respectively. Besides the abundance of microbial species, genotype, diet, and weight were included in the variables, and together they explain $42.0 \%$ of the data. The first and second ordination plots are shown on $x$ - and $y$-axis respectively; the third ordination plot explaining $7.5 \%$ is not shown. Both genotype and diet had a significant impact on the microbial composition $(p<0.05)$. DR was started at the age of 7 -wk $(10 \%)$, increasing to $30 \%$ after two weeks. DR control animals had free access to food. TrpR was started at the age of 4-wk (95\%), combined with $10 \%$ DR. TrpR control animals received $10 \%$ DR. All animals were sacrificed at 16-wk age. Data represent $n=4 \operatorname{Ercc}^{-1 / \Delta 7}$ mice and $n=3 \operatorname{Ercc}^{1 /+}$ mice, expressed as mean + SEM. ${ }^{*}=p<0.05 ;{ }^{* *}=p<0.01$, as determined by Student's unpaired $t$ test. $B M=$ bone marrow; $D R=$ dietary restriction; TrpR = tryptophan restriction. Van Beek et al, unpublished findings. 
Chapter 9

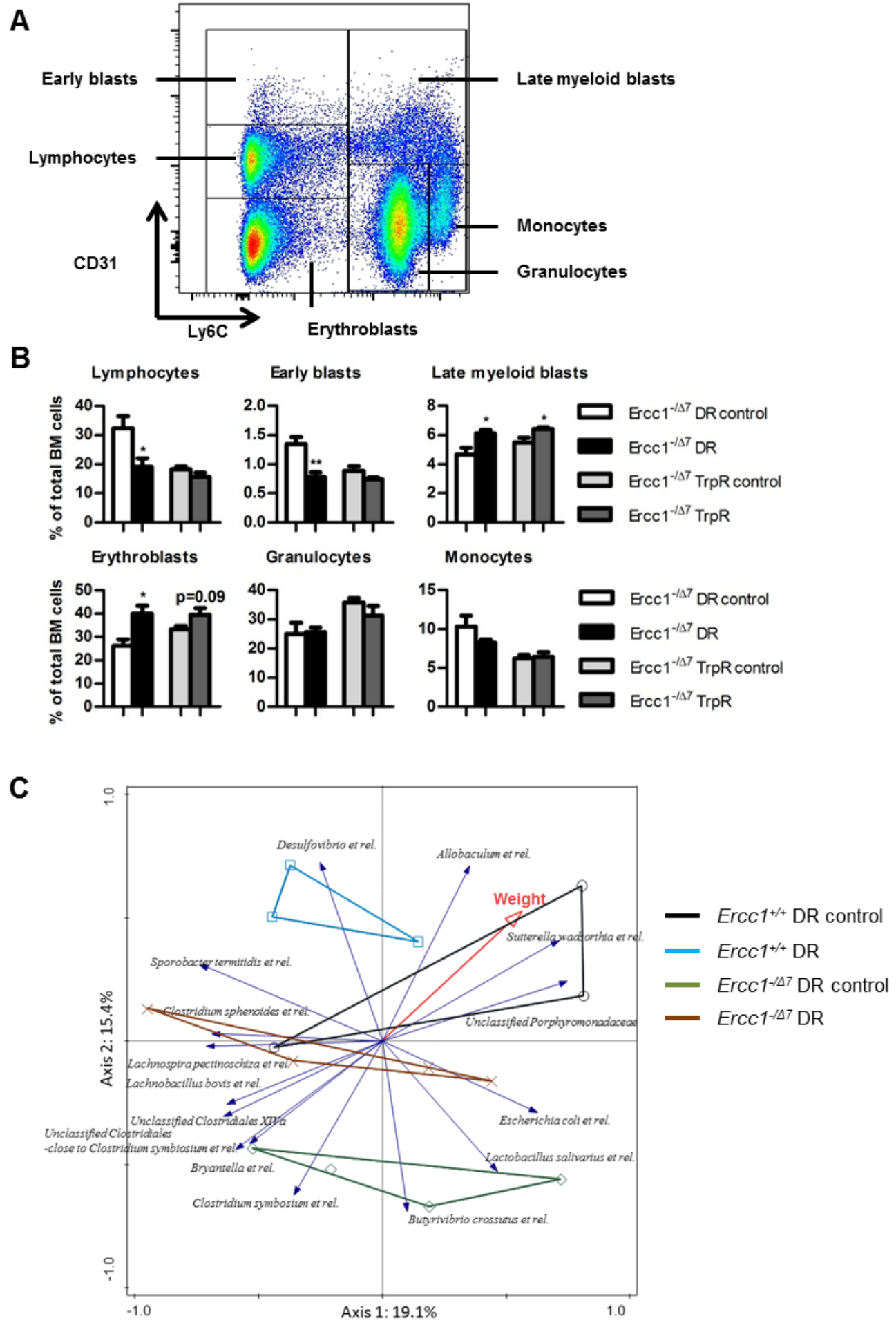




\section{Aging mouse model: expedite testing of nutritional interventions for the elderly}

In our studies to candidate probiotics and dietary tryptophan restriction, we have used the accelerated aging Ercc $1^{-/ \Delta 7}$ mouse model. Of the various accelerated aging models that exist, this model has been described to develop the broadest spectrum of agerelated phenotypes and pathologies (Table 1; [62, 495, 668]). Our data demonstrate that the age-related decline in the mucus barrier of normal aging mice is also present in $E r c c 1^{-1 \Delta 7}$ mice (chapter 6). Moreover, we have found that in many aspects, the effect of accelerated aging on the distribution of immune cells in $E r c c 1^{-1 \Delta 7}$ mice resembles that of normal aging mice (chapter 7 ). Thus, we provided evidence that $E r c c 1^{-/ \Delta 7}$ mice manifest two additional aspects of genuine aging.

Table 1. Aging phenomena in $\operatorname{Ercc1}^{-/ \Delta 7}$ mice.

\begin{tabular}{|c|c|c|}
\hline Organ & Symptoms & Acceleration? $^{1}$ \\
\hline Bone & Kyphosis/osteoporosis & $=$ \\
\hline \multirow{2}{*}{ Bone marrow } & $\downarrow$ B cell precursors & $=$ \\
\hline & Fatty infiltration & $\uparrow$ \\
\hline \multirow[t]{2}{*}{ Brain/CNS } & Vacuolization & $\downarrow$ \\
\hline & Atrophy/ataxia & $\uparrow$ \\
\hline \multirow[t]{3}{*}{ Gut } & Inflammatory pathways & $={ }^{*}$ \\
\hline & $\downarrow$ Mucus layer & $=$ \\
\hline & $\downarrow$ Compartmentalization bacteria - epithelium & $=$ \\
\hline Heart & Myocardial degeneration & $=$ \\
\hline \multirow[t]{2}{*}{ Kidney } & Tubular degeneration & $=$ \\
\hline & Anisokaryosis & $\uparrow$ \\
\hline \multirow{2}{*}{ Liver } & Anisokaryosis/lipofuscin & $=$ \\
\hline & Intranuclear inclusions & $\uparrow$ \\
\hline MLN & $\uparrow \mathrm{B}$ cells & $=$ \\
\hline Muscles & Sarcopenia & $=$ \\
\hline Peritoneal cavity & $\uparrow$ Cell counts/B1 cells & $\downarrow^{*}$ \\
\hline Skin & Atrophy/hair graying & $\downarrow$ \\
\hline \multirow[t]{4}{*}{ Spleen } & $\downarrow$ Naïve $/ \uparrow$ memory T cells & $=$ \\
\hline & $\uparrow$ Treg & $=$ \\
\hline & $\downarrow$ NK cells & $\uparrow$ \\
\hline & Atrophy & $\uparrow$ \\
\hline Testis & Tubular degeneration & $\uparrow$ \\
\hline Thymus & Involution/T cell maturation & $=$ \\
\hline
\end{tabular}

'As compared with normal aging mice (on a biological age scale). *Van Beek et al, unpublished findings. $\downarrow$ less pronounced in $\operatorname{Ercc~}^{-1 / 47}$ mice than in normal aged mice; = comparable with normal aged mice; $\uparrow$ more pronounced than in normal aged mice. Based on findings by Dollé et al (2011), Gurkar and Niedernhofer (2015), and findings in this thesis. CNS = central nervous system; MLN = mesenteric lymph nodes.

More importantly, we show that the $E r c c 1^{-1 / 47}$ model is useful in expedite testing of nutritional interventions. We showed that bacterial supplementations and dietary tryptophan restriction changed the phenotype of the intestinal barrier and/or immune system in $E r c c 1^{-1 / 47}$ mice. Lifespan studies in $E r c c 1^{-1 / 47}$ mice enable to screen for life-extending dietary components or regimes in a relatively fast and sensitive manner.

Future directions: In the context of microbial changes upon nutritional interventions, it would be interesting to evaluate these nutritional interventions in germfree $E r c c 1^{-1 / 4}$ mice. It would be novel to breed germfree $E r c c 1^{-1 \Delta 7}$ mice, and this would enable to gain insight into the effect of absence of gut microbiota in aging. Keeping germfree 
mice for more than 18 months is time-consuming and expensive. Generating germfree Ercc1 ${ }^{-/ \Delta 7}$ mice would also give possibilities to transfer microbiota from young or old mice to aged germfree $E r c c 1^{-1 / 7}$ mice in order to gain insight into the role that young microbiota may play in the (accelerated) aging gut. Furthermore, mono-colonizing germfree mice with L. plantarum WCFS1 would give insight into the sole effect of this bacterial strain in the aging gut. The application of challenge protocols in aging and nutrition research is crucial. We described the use of a challenge with a model antigen (TNP-KLH, chapter 7) to assess antibody production in T cell-dependent B cell responses. Challenges in the gut, where food components enter the body, are of specific interest in nutritional interventions. We have performed a pilot study in which we infected 8-week-old $\operatorname{Ercc}^{-1 \Delta 7}$ mice $(\mathrm{n}=6)$ with Salmonella enteritidis (data not shown), which in WT mice translocates to (a.o.) MLN, liver, and spleen [669]. After 4 days of infection, we found very low to undetectable counts of Salmonella in various organs $(n=3)$, whereas one mouse had very high levels of Salmonella after 7 days of infection $\left(2.3 \times 10^{3}-4.5 \times 10^{3} \mathrm{CFU} / \mathrm{mg} \mathrm{MLN}\right.$, liver, spleen, and ileum). Thus, further optimization of such challenge protocols is required to be applicable in $\operatorname{Ercc}^{-1 \Delta 7}$ mice. It can then be used to study whether e.g. L. plantarum WCFS1 confers a health benefit on Salmonella-infected $E r c c 1^{-1 \Delta 7}$ mice. Other examples of challenges that have direct relevance for elderly are influenza and cytomegalovirus (CMV).

Predictive, preventive and personalized nutrition to modulate a person's health is a fruitful, but challenging topic for research [670]. Immunonutrition seems to be of particular interest for the aging immune system. As we showed that the aging condition of the intestinal barrier and immune system plays an important role in the effect of nutritional intervention (chapter 6), this suggests that nutritional interventions cannot be safely applied to the whole population (young-aged, healthy-diseased) without thorough testing for different age or health conditions.

\section{Concluding remarks}

The studies described in this thesis show the effect of aging on the mouse immune system and microbiota composition (Figure 1). Moreover, two interventions (probiotics, dietary tryptophan restriction) have been applied in mice and were shown to affect the aging immune system and microbiota (chapter 6 and 7). Also, a role for aged microbiota on the immune system has been established by using germfree mice (chapter 8 ). Finally, these findings are integrated in this chapter, and put in perspective of further research.

Further research in human subjects is needed to translate our findings regarding probiotics. We suggest that only those bacterial strains that have shown beneficial effects in aged mice can be tested for in vivo application in elderly. Vice versa, it would be of 
great interest to apply commercially available probiotics such as Lactobacillus casei strain Shirota (LcS), Lactobacillus rhamnosus Gorbach and Goldin (LGG), LactobacilIus acidophilus NCFM, or Lactobacillus johnsonii La1 in the Ercc ${ }^{-1 \Delta 7}$ model. In addition, because life extension has been shown for supplementation with Bifidobacterium animalis LK512 [165], it would be interesting to test whether this bacterial strain extends the life of $E r C c 1^{-1 \Delta 7}$ mice.

In further pursue of healthy aging, nutritional interventions play an important role. It is, however, important to gain insight into the mechanisms whereby nutritional interventions modulate the intestinal barrier and the immune system to refine the search for appropriate nutritional interventions in the context of aging. The ultimate aim is to extend the health span of elderly by application of probiotics or other defined nutritional components, or by restriction of food components. 


\section{References}

1. Hetz, C. (2012) The unfolded protein response: controlling cell fate decisions under ER stress and beyond. Nat Rev Mol Cell Biol 13, 89-102.

2. Hotamisligil, G. S. (2010) Endoplasmic reticulum stress and the inflammatory basis of metabolic disease. Cell 140, 900-17.

3. Zhou, Y., Lee, J., Reno, C. M., Sun, C., Park, S. W., Chung, J., Lee, J., Fisher, S. J., White, M. F., Biddinger, S. B., Ozcan, U. (2011) Regulation of glucose homeostasis through a XBP-1-FoxO1 interaction. Nat Med 17, 35665.

4. Wang, H., Dwyer-Lindgren, L., Lofgren, K. T., Rajaratnam, J. K., Marcus, J. R., Levin-Rector, A., Levitz, C. E., Lopez, A. D., Murray, C. J. (2013) Agespecific and sex-specific mortality in 187 countries, 1970-2010: a systematic analysis for the Global Burden of Disease Study 2010. The Lancet 380, 20712094.

5. (2010) World Health Statistics 2010. WHO Press, Geneva 166.

6. Kroemer, G., Marino, G., Levine, B. (2010) Autophagy and the integrated stress response. Mol Cell 40, 280-93.

7. Kato, H., Nakajima, S., Saito, Y., Takahashi, S., Katoh, R., Kitamura, M. (2012) mTORC1 serves ER stress-triggered apoptosis via selective activation of the IRE1-JNK pathway. Cell Death Differ 19, 310-20.

8. Martinon, F., Chen, X., Lee, A. H., Glimcher, L. H. (2010) TLR activation of the transcription factor XBP1 regulates innate immune responses in macrophages. Nat Immunol 11, 411-8.

9. Oh, J., Riek, A. E., Weng, S., Petty, M., Kim, D., Colonna, M., Cella, M., Bernal-Mizrachi, C. (2012) Endoplasmic reticulum stress controls M2 macrophage differentiation and foam cell formation. J Biol Chem 287, 1162941.

10. Ghosh, A. K., Garg, S. K., Mau, T., O'Brien, M., Liu, J., Yung, R. (2015) Elevated Endoplasmic Reticulum Stress Response Contributes to Adipose Tissue Inflammation in Aging. J Gerontol A Biol Sci Med Sci 70, 1320-9.

11. López-Otín, C., Blasco, M. A., Partridge, L., Serrano, M., Kroemer, G. (2013) The hallmarks of aging. Cell 153, 1194-1217.

12. Malaguarnera, L., Ferlito, L., Imbesi, R., Gulizia, G., Di Mauro, S., Maugeri, D., Malaguarnera, M., Messina, A. (2001) Immunosenescence: a review. Archives of Gerontology and Geriatrics 32, 1-14.

13. Wick, G. and Grubeck-Loebenstein, B. (1997) The aging immune system: primary and secondary alterations of immune reactivity in the elderly. Experimental Gerontology 32, 401-413.

14. Lozano, R., Naghavi, M., Foreman, K., Lim, S., Shibuya, K., Aboyans, V., Abraham, J., Adair, T., Aggarwal, R., Ahn, S. Y. (2013) Global and regional mortality from 235 causes of death for 20 age groups in 1990 and 2010: a systematic analysis for the Global Burden of Disease Study 2010. The Lancet $380,2095-2128$.

15. Naidoo, N. (2009) ER and aging-Protein folding and the ER stress response. Ageing Res Rev 8, 150-9.

16. Tang, Y., Purkayastha, S., Cai, D. (2015) Hypothalamic microinflammation: a common basis of metabolic syndrome and aging. Trends Neurosci 38, 36-44.

17. CDC (2013) The state of aging and health in America 2013. Volume 2016, Atlanta, GA. 
18. Heidenreich, P. A., Trogdon, J. G., Khavjou, O. A., Butler, J., Dracup, K., Ezekowitz, M. D., Finkelstein, E. A., Hong, Y., Johnston, S. C., Khera, A. (2011) Forecasting the future of cardiovascular disease in the United States a policy statement from the American heart association. Circulation 123, 933944.

19. (2011) Global Atlas on Cardiovascular Disease Prevention and Control. World Health Organization., Geneva.

20. Baumeister, S. H., Freeman, G. J., Dranoff, G., Sharpe, A. H. (2016) Coinhibitory Pathways in Immunotherapy for Cancer. Annual Review of Immunology 34, 539-573.

21. Mariotto, A. B., Yabroff, K. R., Shao, Y., Feuer, E. J., Brown, M. L. (2011) Projections of the cost of cancer care in the United States: 2010-2020. Journal of the National Cancer Institute 103, 117-128.

22. Anand, P., Kunnumakara, A. B., Sundaram, C., Harikumar, K. B., Tharakan, S. T., Lai, O. S., Sung, B., Aggarwal, B. B. (2008) Cancer is a preventable disease that requires major lifestyle changes. Pharmaceutical Research 25, 2097-2116.

23. Guarascio, A. J., Ray, S. M., Finch, C. K., Self, T. H. (2013) The clinical and economic burden of chronic obstructive pulmonary disease in the USA. ClinicoEconomics and outcomes research: CEOR 5, 235-245.

24. Chung, K. and Adcock, I. (2008) Multifaceted mechanisms in COPD: inflammation, immunity, and tissue repair and destruction. European Respiratory Journal 31, 1334-1356.

25. Stöhr, J., Condello, C., Watts, J. C., Bloch, L., Oehler, A., Nick, M., DeArmond, S. J., Giles, K., DeGrado, W. F., Prusiner, S. B. (2014) Distinct synthetic $A \beta$ prion strains producing different amyloid deposits in bigenic mice. Proceedings of the National Academy of Sciences 111, 10329-10334.

26. Watts, J. C., Condello, C., Stöhr, J., Oehler, A., Lee, J., DeArmond, S. J., Lannfelt, L., Ingelsson, M., Giles, K., Prusiner, S. B. (2014) Serial propagation of distinct strains of $A \beta$ prions from Alzheimer's disease patients. Proceedings of the National Academy of Sciences 111, 10323-10328.

27. Association, A. S. (2015) 2015 Alzheimer's disease facts and figures. Alzheimer's \& dementia: the journal of the Alzheimer's Association 11, 332 384.

28. Mosher, K. I. and Wyss-Coray, T. (2014) Microglial dysfunction in brain aging and Alzheimer's disease. Biochemical Pharmacology 88, 594-604.

29. Franco, O. H., Steyerberg, E. W., Hu, F. B., Mackenbach, J., Nusselder, W. (2007) Associations of diabetes mellitus with total life expectancy and life expectancy with and without cardiovascular disease. Archives of Internal Medicine 167, 1145-1151.

30. Shin, J. J., Lee, E. K., Park, T. J., Kim, W. (2015) Damage-associated molecular patterns and their pathological relevance in diabetes mellitus. Ageing Research Reviews 24, 66-76.

31. CDC (2014) Diabetes Report Card 2014. Volume 2016Centers for Disease Control and Prevention, Atlanta, GA.

32. Hirsch, E. C. and Hunot, S. (2009) Neuroinflammation in Parkinson's disease: a target for neuroprotection? The Lancet Neurology 8, 382-397.

33. O'Brien, J., Ward, A., Michels, S., Tzivelekis, S., Brandt, N. (2009) Economic burden associated with Parkinson disease. Drug Benefit Trends 21, 179-190. 
34. CDC (2007) National and state medical expenditures and lost earnings attributable to arthritis and other rheumatic conditions---United States, 2003. Morbidity and Mortality Weekly Report 56, 4-7.

35. Mobasheri, A. and Henrotin, Y. (2015) Biomarkers of (osteo) arthritis. Biomarkers 20, 513-518.

36. Ginaldi, L., Di Benedetto, M. C., De Martinis, M. (2005) Osteoporosis, inflammation and ageing. Immunity \& Ageing 2, 14.

37. Blume, S. W. and Curtis, J. (2011) Medical costs of osteoporosis in the elderly Medicare population. Osteoporosis International 22, 1835-1844.

38. Janssen, I., Shepard, D. S., Katzmarzyk, P. T., Roubenoff, R. (2004) The healthcare costs of sarcopenia in the United States. Journal of the American Geriatrics Society 52, 80-85.

39. Beaudart, C., Rizzoli, R., Bruyère, O., Reginster, J.-Y., Biver, E. (2014) Sarcopenia: burden and challenges for public health. Archives of Public Health 72, 1-8.

40. Fielding, R. A., Vellas, B., Evans, W. J., Bhasin, S., Morley, J. E., Newman, A. B., van Kan, G. A., Andrieu, S., Bauer, J., Breuille, D. (2011) Sarcopenia: an undiagnosed condition in older adults. Current consensus definition: prevalence, etiology, and consequences. International working group on sarcopenia. Journal of the American Medical Directors Association 12, 249256.

41. Maue, A. C. and Haynes, L. (2009) CD4+ T Cells and Immunosenescence-A Mini-Review. Gerontology 55, 491-495.

42. Henson, S. M. and Akbar, A. N. (2010) Memory T-Cell Homeostasis and Senescence during Aging. Advances in Experimental Medicine and Biology 684, 189-197.

43. Gavazzi, G. and Krause, K. H. (2002) Ageing and infection. The Lancet Infectious Diseases 2, 659-666.

44. Beard, J., Biggs, S., Bloom, D. E., Fried, L. P., Hogan, P. R., Kalache, A., Olshansky, S. J. (2012) Global population ageing: Peril or promise? Program on the Global Demography of Aging.

45. Sahin, E. and DePinho, R. A. (2010) Linking functional decline of telomeres, mitochondria and stem cells during ageing. Nature 464, 520-528.

46. Effros, R. B. (2007) Telomerase induction in T cells: A cure for aging and disease? Experimental Gerontology 42, 416-420.

47. Sahin, E., Colla, S., Liesa, M., Moslehi, J., Müller, F. L., Guo, M., Cooper, M., Kotton, D., Fabian, A. J., Walkey, C. (2011) Telomere dysfunction induces metabolic and mitochondrial compromise. Nature 470, 359-365.

48. Signer, R. A. J., Montecino-Rodriguez, E., Witte, O. N., Dorshkind, K. (2008) Aging and cancer resistance in lymphoid progenitors are linked processes conferred by p16Ink4a and Arf. Genes \& Development 22, 3115-3120.

49. Dorshkind, K., Montecino-Rodriguez, E., Signer, R. A. J. (2009) The ageing immune system: is it ever too old to become young again? Nature Reviews Immunology 9, 57-62.

50. De la Fuente, M. (2008) Role of neuroimmunomodulation in aging. Neuroimmunomodulation 15, 213-223.

51. Barzilai, A. and Yamamoto, K.-I. (2004) DNA damage responses to oxidative stress. DNA repair 3, 1109-1115.

52. Signer, R. A. and Morrison, S. J. (2013) Mechanisms that regulate stem cell aging and life span. Cell Stem Cell 12, 152-165. 
53. Fernández Morera, J., Calvanese, V., Rodríguez Rodero, S., Menéndez Torre, E., Fraga, M. (2010) Epigenetic regulation of the immune system in health and disease. Tissue Antigens 76, 431-439.

54. Calvanese, V., Lara, E., Kahn, A., Fraga, M. F. (2009) The role of epigenetics in aging and age-related diseases. Ageing Research Reviews 8, 268-276.

55. Min, H., Montecino-Rodriguez, E., Dorshkind, K. (2006) Effects of aging on the common lymphoid progenitor to pro-B cell transition. The Journal of Immunology 176, 1007-1012.

56. Fraga, M. F., Ballestar, E., Paz, M. F., Ropero, S., Setien, F., Ballestar, M. L., Heine-Suñer, D., Cigudosa, J. C., Urioste, M., Benitez, J. (2005) Epigenetic differences arise during the lifetime of monozygotic twins. Proceedings of the National Academy of Sciences 102, 10604-10609.

57. Brodin, P., Jojic, V., Gao, T., Bhattacharya, S., Angel, C. J. L., Furman, D., Shen-Orr, S., Dekker, C. L., Swan, G. E., Butte, A. J. (2015) Variation in the Human Immune System Is Largely Driven by Non-Heritable Influences. Cell 160, 37-47.

58. Vermeij, W. P., Hoeijmakers, J., Pothof, J. (2016) Genome Integrity in Aging: Human Syndromes, Mouse Models, and Therapeutic Options. Annual Review of Pharmacology and Toxicology 56, 427-445.

59. Gregg, S. Q., Robinson, A. R., Niedernhofer, L. J. (2011) Physiological consequences of defects in ERCC1-XPF DNA repair endonuclease. DNA repair $10,781-791$.

60. Weeda, G., Donker, I., de Wit, J., Morreau, H., Janssens, R., Vissers, C., Nigg, A., van Steeg, H., Bootsma, D., Hoeijmakers, J. (1997) Disruption of mouse ERCC1 results in a novel repair syndrome with growth failure, nuclear abnormalities and senescence. Current Biology 7, 427-439.

61. McWhir, J., Selfridge, J., Harrison, D. J., Squires, S., Melton, D. W. (1993) Mice with DNA repair gene (ERCC-1) deficiency have elevated levels of p53, liver nuclear abnormalities and die before weaning. Nature Genetics 5, 217224.

62. Dollé, M. E., Kuiper, R. V., Roodbergen, M., Robinson, J., de Vlugt, S., Wijnhoven, S. W., Beems, R. B., de la Fonteyne, L., de With, P., van der Pluijm, I. (2011) Broad segmental progeroid changes in short-lived Ercc1-/ $\Delta 7$ mice. Pathobiology of Aging \& Age-related Diseases 1.

63. Wessels, I., Jansen, J., Rink, L., Uciechowski, P. (2010) Immunosenescence of polymorphonuclear neutrophils. TheScientificWorldJournal 10, 145-160.

64. Geiger, H., de Haan, G., Florian, M. C. (2013) The ageing haematopoietic stem cell compartment. Nature Reviews Immunology 13, 376-389.

65. Chambers, S. M., Shaw, C. A., Gatza, C., Fisk, C. J., Donehower, L. A., Goodell, M. A. (2007) Aging hematopoietic stem cells decline in function and exhibit epigenetic dysregulation. PLoS Biology 5, e201.

66. Lindstrom, T. M. and Robinson, W. H. (2010) Rheumatoid Arthritis: A Role for Immunosenescence? Journal of the American Geriatrics Society 58, 15651575.

67. Rossi, D. J., Bryder, D., Zahn, J. M., Ahlenius, H., Sonu, R., Wagers, A. J., Weissman, I. L. (2005) Cell intrinsic alterations underlie hematopoietic stem cell aging. Proceedings of the National Academy of Sciences 102, 9194-9199.

68. Warren, L. A. and Rossi, D. J. (2009) Stem cells and aging in the hematopoietic system. Mechanisms of Ageing and Development 130, 46-53.

69. Ershler, W., Sun, W., Binkley, N., Gravenstein, S., Volk, M., Kamoske, G., Klopp, R., Roecker, E., Daynes, R., Weindruch, R. (1993) Interleukin-6 and 
aging: blood levels and mononuclear cell production increase with advancing age and in vitro production is modifiable by dietary restriction. Lymphokine and Cytokine Research 12, 225-230.

70. Harris, T. B., Ferrucci, L., Tracy, R. P., Corti, M. C., Wacholder, S., Ettinger, W. H., Heimovitz, H., Cohen, H. J., Wallace, R. (1999) Associations of elevated interleukin-6 and C-reactive protein levels with mortality in the elderly. The American Journal of Medicine 106, 506-512.

71. Franceschi, C., Bonafè, M., Valensin, S., Olivieri, F., de Luca, M., Ottaviani, E., de Benedictis, G. (2000) Inflamm-aging: an evolutionary perspective on immunosenescence. Annals of the New York Academy of Sciences 908, 244254.

72. Gomez, C. R., Boehmer, E. D., Kovacs, E. J. (2005) The aging innate immune system. Current Opinion in Immunology 17, 457-462.

73. Valiathan, R., Ashman, M., Asthana, D. (2016) Effects of Ageing on the Immune System: Infants to Elderly. Scandinavian Journal of Immunology 83, 255-266.

74. Strindhall, J., Nilsson, B. O., Löfgren, S., Ernerudh, J., Pawelec, G., Johansson, B., Wikby, A. (2007) No immune risk profile among individuals who reach 100 years of age: findings from the Swedish NONA immune longitudinal study. Experimental Gerontology 42, 753-761.

75. Agarwal, S. and Busse, P. J. (2010) Innate and adaptive immunosenescence. Annals of Allergy, Asthma \& Immunology 104, 183-190.

76. Pawelec, G. and Larbi, A. (2008) Immunity and ageing in man: Annual Review 2006/2007. Experimental Gerontology 43, 34-38.

77. Simell, B., Vuorela, A., Ekstrom, N., Palmu, A., Reunanen, A., Meri, S., Kayhty, H., Vakevainen, M. (2011) Aging reduces the functionality of antipneumococcal antibodies and the killing of Streptococcus pneumoniae by neutrophil phagocytosis. Vaccine 29, 1929-1934.

78. Mathur, S. K., Schwantes, E. A., Jarjour, N. N., Busse, W. W. (2008) AgeRelated Changes in Eosinophil Function in Human Subjects. Chest 133, 412 419 .

79. Marone, G., Poto, S., di Martino, L., Condorelli, M. (1986) Human basophil releasability: I. Age-related changes in basophil releasability. Journal of Allergy and Clinical Immunology 77, 377-383.

80. Merino, A., Buendia, P., Martin-Malo, A., Aljama, P., Ramirez, R., Carracedo, J. (2011) Senescent CD14+ CD16+ monocytes exhibit proinflammatory and proatherosclerotic activity. The Journal of Immunology 186, 1809-1815.

81. Lee, N., Shin, M. S., Kang, K. S., Yoo, S.-A., Mohanty, S., Montgomery, R. R., Shaw, A. C., Kang, I. (2014) Human monocytes have increased IFN-Ymediated IL-15 production with age alongside altered IFN-ץ receptor signaling. Clinical Immunology 152, 101-110.

82. Verschoor, C. P., Johnstone, J., Millar, J., Parsons, R., Lelic, A., Loeb, M., Bramson, J. L., Bowdish, D. M. (2014) Alterations to the frequency and function of peripheral blood monocytes and associations with chronic disease in the advanced-age, frail elderly. PLoS ONE 9, e104522.

83. van Duin, D., Mohanty, S., Thomas, V., Ginter, S., Montgomery, R. R., Fikrig, E., Allore, H. G., Medzhitov, R., Shaw, A. C. (2007) Age-associated defect in human TLR-1/2 function. The Journal of Immunology 178, 970-975. 
84. Shaw, A. C., Panda, A., Joshi, S. R., Qian, F., Allore, H. G., Montgomery, R. R. (2011) Dysregulation of human Toll-like receptor function in aging. Ageing Research Reviews 10, 346-353.

85. Seidler, S., Zimmermann, H. W., Bartneck, M., Trautwein, C., Tacke, F. (2010) Age-dependent alterations of monocyte subsets and monocyte-related chemokine pathways in healthy adults. BMC Immunology 11, 30.

86. Hearps, A. C., Martin, G. E., Angelovich, T. A., Cheng, W. J., Maisa, A., Landay, A. L., Jaworowski, A., Crowe, S. M. (2012) Aging is associated with chronic innate immune activation and dysregulation of monocyte phenotype and function. Aging Cell 11, 867-875.

87. Krasselt, M., Baerwald, C., Wagner, U., Rossol, M. (2013) CD56+ monocytes have a dysregulated cytokine response to lipopolysaccharide and accumulate in rheumatoid arthritis and immunosenescence. Arthritis Research\&Therapy 15, R139.

88. Nyugen, J., Agrawal, S., Gollapudi, S., Gupta, S. (2010) Impaired functions of peripheral blood monocyte subpopulations in aged humans. Journal of Clinical Immunology 30, 806-813.

89. Spits, H., Artis, D., Colonna, M., Diefenbach, A., Di Santo, J. P., Eberl, G., Koyasu, S., Locksley, R. M., McKenzie, A. N., Mebius, R. E. (2013) Innate lymphoid cells-a proposal for uniform nomenclature. Nature Reviews Immunology 13, 145-149.

90. Keren, Z., Naor, S., Nussbaum, S., Golan, K., Itkin, T., Sasaki, Y., SchmidtSupprian, M., Lapidot, T., Melamed, D. (2011) B cell depletion reactivates B lymphopoiesis in the BM and rejuvenates the B lineage in aging. Blood 117, 3104-3112.

91. Frasca, D., Diaz, A., Romero, M., Landin, A. M., Blomberg, B. B. (2011) Age effects on $B$ cells and humoral immunity in humans. Ageing Research Reviews 10, 330-335.

92. Ademokun, A., Wu, Y. C., Dunn-Walters, D. (2010) The ageing B cell population: Composition and function. Biogerontology 11, 125-137.

93. Frasca, D., Landin, A. M., Lechner, S. C., Ryan, J. G., Schwartz, R., Riley, R. L., Blomberg, B. B. (2008) Aging down-regulates the transcription factor E2A, activation-induced cytidine deaminase, and Ig class switch in human B cells. The Journal of Immunology 180, 5283-5290.

94. Listi, F., Candore, G., Modica, M. A., Russo, M., Di Lorenzo, G., EspositoPellitteri, M., Colonna-Romano, G., Aquino, A., Bulati, M., Lio, D. (2006) A study of serum immunoglobulin levels in elderly persons that provides new insights into B cell immunosenescence. Annals of the New York Academy of Sciences 1089, 487-495.

95. Colonna-Romano, G., Aquino, A., Bulati, M., Lorenzo, G. D., Listì, F., Vitello, S., Lio, D., Candore, G., Clesi, G., Caruso, C. (2006) Memory B cell subpopulations in the aged. Rejuvenation Research 9, 149-152.

96. Gibson, K. L., Wu, Y. C., Barnett, Y., Duggan, O., Vaughan, R., Kondeatis, E., Nilsson, B. O., Wikby, A., Kipling, D., Dunn Walters, D. K. (2009) B cell diversity decreases in old age and is correlated with poor health status. Aging Cell 8, 18-25.

97. Frasca, D. and Blomberg, B. B. (2011) Aging affects human B cell responses. Journal of Clinical Immunology 31, 430-435.

98. Tachikawa, S., Kawamura, T., Kawamura, H., Kanda, Y., Fujii, Y., Matsumoto, H., Abo, T. (2008) Appearance of B220low autoantibody producing B 1 cells 
at neonatal and older stages in mice. Clinical \& Experimental Immunology $153,448-455$.

99. Howard, W. A., Gibson, K. L., Dunn-Walters, D. K. (2006) Antibody quality in old age. Rejuvenation Research 9, 117-125.

100. Dunn-Walters, D. K. and Ademokun, A. A. (2010) B cell repertoire and ageing. Current Opinion in Immunology 22, 514-520.

101. Yanaba, K., Bouaziz, J. D., Haas, K. M., Poe, J. C., Fujimoto, M., Tedder, T. F. (2008) A regulatory B cell subset with a unique CD1dhiCD5+ phenotype controls T cell-dependent inflammatory responses. Immunity 28, 639-650.

102. Lund, F. E. and Randall, T. D. (2010) Effector and regulatory B cells: modulators of CD4+ T cell immunity. Nature Reviews Immunology 10, 236247.

103. Twohig, J. P., Pappworth, I. Y., Sivasankar, B., Kulik, L., Bull, M., Holers, V. M., Wang, E. C. Y., Marchbank, K. J. (2009) Defective B cell ontogeny and humoral immune response in mice prematurely expressing human complement receptor 2 (CR2, CD21) is similar to that seen in aging wild type mice. Molecular Immunology 46, 2002-2013.

104. Aspinall, R., Pitts, D., Lapenna, A., Mitchell, W. (2010) Immunity in the elderly: the role of the thymus. Journal of Comparative Pathology 142, S111-S115.

105. Arnold, C. R., Wolf, J., Brunner, S., Herndler-Brandstetter, D., GrubeckLoebenstein, B. (2011) Gain and Loss of T Cell Subsets in Old Age-AgeRelated Reshaping of the T Cell Repertoire. Journal of Clinical Immunology 31, 137-146.

106. Sauce, D., Larsen, M., Fastenackels, S., Duperrier, A., Keller, M., GrubeckLoebenstein, B., Ferrand, C., Debré, P., Sidi, D., Appay, V. (2009) Evidence of premature immune aging in patients thymectomized during early childhood. The Journal of Clinical Investigation 119, 3070-3078.

107. Biagi, E., Nylund, L., Candela, M., Ostan, R., Bucci, L., Pini, E., Nikkïla, J., Monti, D., Satokari, R., Franceschi, C. (2010) Through ageing, and beyond: gut microbiota and inflammatory status in seniors and centenarians. PLoS ONE 5, e10667.

108. Crooks, C. V., Cross, M. L., Wall, C. R. (2010) Age-related differences in integrin expression in peripheral blood lymphocytes. Immunity \& Ageing 7:5.

109. Yan, J., Greer, J. M., Hull, R., O'Sullivan, J. D., Henderson, R. D., Read, S. J., McCombe, P. A. (2010) The effect of ageing on human lymphocyte subsets: comparison of males and females. Immunity \& Ageing 7:4.

110. Pereira, L. F., Duarte de Souza, A. P., Borges, T. J., Bonorino, C. (2011) Impaired in vivo CD4+ T cell expansion and differentiation in aged mice is not solely due to $T$ cell defects: Decreased stimulation by aged dendritic cells. Mechanisms of Ageing and Development 132, 187-194.

111. Quadri, R. A., Plastre, O., Phelouzat, M. A., Arbogast, A., Proust, J. J. (1996) Age-related tyrosine-specific protein phosphorylation defect in human $\mathrm{T}$ lymphocytes activated through CD3, CD4, CD8 or the IL-2 receptor. Mechanisms of Ageing and Development 88, 125-138.

112. Alonso-Arias, R., Moro-García, M. A., López-Vázquez, A., Rodrigo, L., Baltar, J., García, F. M. S., Jaurrieta, J. J. S., López-Larrea, C. (2011) NKG2D expression in CD4+ $\mathrm{T}$ lymphocytes as a marker of senescence in the aged immune system. AGE 33, 591-605.

113. Leng, S. X., Qu, T., Semba, R. D., Li, H., Yao, X., Nilles, T., Yang, X., Manwani, B., Walston, J. D., Ferrucci, L. (2011) Relationship between cytomegalovirus (CMV) IgG serology, detectable CMV DNA in peripheral 
monocytes, and CMV pp65 495-503-specific CD8+ T cells in older adults. AGE 33, 607-614.

114. Jiang, J., Fisher, E. M., Murasko, D. M. (2011) CD8 T cell responses to influenza virus infection in aged mice. Ageing Research Reviews 10, 422-427.

115. Arens, R., Loewendorf, A., Redeker, A., Sierro, S., Boon, L., Klenerman, P., Benedict, C. A., Schoenberger, S. P. (2011) Differential B7-CD28 Costimulatory Requirements for Stable and Inflationary Mouse Cytomegalovirus-Specific Memory CD8 T Cell Populations. The Journal of Immunology 186, 3874-3881.

116. Pawelec, G., Akbar, A., Beverley, P., Caruso, C., Derhovanessian, E., Fülöp, T., Griffiths, P., Grubeck-Loebenstein, B., Hamprecht, K., Jahn, G. (2010) Immunosenescence and Cytomegalovirus: where do we stand after a decade? Immunity \& Ageing 7:13.

117. Vukmanovic-Stejic, M., Zhang, Y., Cook, J. E., Fletcher, J. M., McQuaid, A., Masters, J. E., Rustin, M. H. A., Taams, L. S., Beverley, P. C. L., Macallan, D. C. (2006) Human CD4 CD25 hi Foxp3 regulatory T cells are derived by rapid turnover of memory populations in vivo. The Journal of Clinical Investigation 116, 2423-2433.

118. Derhovanessian, E., Maier, A. B., Beck, R., Jahn, G., Hähnel, K., Slagboom, P. E., de Craen, A. J. M., Westendorp, R. G. J., Pawelec, G. (2010) Hallmark Features of Immunosenescence Are Absent in Familial Longevity. The Journal of Immunology 185, 4618-4624.

119. Annunziato, F. and Romagnani, S. (2009) Heterogeneity of human effector CD4+ T cells. Arthritis Research and Therapy 11.

120. Belkaid, Y. (2007) Regulatory T cells and infection: a dangerous necessity. Nature Reviews Immunology 7, 875-888.

121. Ongrádi, J. and Kövesdi, V. (2010) Factors that may impact on immunosenescence: an appraisal. Immunity \& Ageing 7:7.

122. Wang, L., Xie, Y., Zhu, L. J., Chang, T. T., Mao, Y. Q., Li, J. (2010) An Association between Immunosenescence and CD4+ CD25+ Regulatory T cells: A Systematic Review. Biomedical and Environmental Sciences 23, 327332.

123. Luckey, T. (1972) Introduction to intestinal microecology. The American journal of clinical nutrition 25, 1292-1294.

124. Tlaskalová-Hogenová, H., Štěpánková, R., Kozáková, H., Hudcovic, T., Vannucci, L., Tučková, L., Rossmann, P., Hrnčír, T., Kverka, M., Zákostelská, Z. (2011) The role of gut microbiota (commensal bacteria) and the mucosal barrier in the pathogenesis of inflammatory and autoimmune diseases and cancer: contribution of germ-free and gnotobiotic animal models of human diseases. Cellular \& Molecular Immunology 8, 110-120.

125. Ivanov, I. I. and Littman, D. R. (2011) Modulation of immune homeostasis by commensal bacteria. Current Opinion in Microbiology 14, 106-114.

126. Chu, H. and Mazmanian, S. K. (2013) Innate immune recognition of the microbiota promotes host-microbial symbiosis. Nature Immunology 14, 668675.

127. Pearson, C., Uhlig, H. H., Powrie, F. (2012) Lymphoid microenvironments and innate lymphoid cells in the gut. Trends in Immunology 33, 289-296.

128. Ogra, P. L. (2010) Ageing and its possible impact on mucosal immune responses. Ageing Research Reviews 9, 101-106. 
129. Santiago, A., Fernandes, R., Santos, B., Assis, F., Oliveira, R., Carvalho, C., Faria, A. (2008) Role of mesenteric lymph nodes and aging in secretory IgA production in mice. Cellular Immunology 253, 5-10.

130. Asahi-Ozaki, Y., Yoshikawa, T., Iwakura, Y., Suzuki, Y., Tamura, S., Kurata, T., Sata, T. (2004) Secretory IgA antibodies provide cross protection against infection with different strains of influenza B virus. Journal of Medical Virology 74, 328-335.

131. Fujihashi, K. and Kiyono, H. (2009) Mucosal immunosenescence: new developments and vaccines to control infectious diseases. Trends in Immunology 30, 334-343.

132. Biagi, E., Candela, M., Fairweather-Tait, S., Franceschi, C., Brigidi, P. (2011) Ageing of the human metaorganism: the microbial counterpart. AGE, 1-21.

133. Tiihonen, K., Ouwehand, A. C., Rautonen, N. (2010) Human intestinal microbiota and healthy ageing. Ageing Research Reviews 9, 107-116.

134. Maslowski, K. M. and Mackay, C. R. (2011) Diet, gut microbiota and immune responses. Nature Immunology 12, 5-9.

135. Johansson, M. E., Ambort, D., Pelaseyed, T., Schütte, A., Gustafsson, J. K., Ermund, A., Subramani, D. B., Holmén-Larsson, J. M., Thomsson, K. A., Bergström, J. H. (2011) Composition and functional role of the mucus layers in the intestine. Cellular and Molecular Life Sciences 68, 3635-3641.

136. Van der Sluis, M., De Koning, B. A., De Bruijn, A. C., Velcich, A., Meijerink, J. P., Van Goudoever, J. B., Büller, H. A., Dekker, J., Van Seuningen, I., Renes, I. B. (2006) Muc2-deficient mice spontaneously develop colitis, indicating that MUC2 is critical for colonic protection. Gastroenterology 131, 117-129.

137. Sovran, B., Loonen, L. M., Lu, P., Hugenholtz, F., Belzer, C., Stolte, E. H., Boekschoten, M. V., van Baarlen, P., Kleerebezem, M., de Vos, P., Dekker, J., Renes, I. B., Wells, J. (2015) IL-22-STAT3 pathway plays a key role in the maintenance of ileal homeostasis in mice lacking secreted mucus barrier. Inflammatory bowel diseases 21, 531-542.

138. Shan, M., Gentile, M., Yeiser, J. R., Walland, A. C., Bornstein, V. U., Chen, K., $\mathrm{He}$, B., Cassis, L., Bigas, A., Cols, M. (2013) Mucus enhances gut homeostasis and oral tolerance by delivering immunoregulatory signals. Science 342, 447-453.

139. Nobori, S., Shimizu, A., Okumi, M., Samelson-Jones, E., Griesemer, A., Hirakata, A., Sachs, D. H., Yamada, K. (2006) Thymic rejuvenation and the induction of tolerance by adult thymic grafts. Proceedings of the National Academy of Sciences 103, 19081-19086.

140. Virts, E. L., Phillips, J. A., Thoman, M. L. (2006) A novel approach to thymic rejuvenation in the aged. Rejuvenation Research 9, 134-142.

141. Redelman, D., Welniak, L. A., Taub, D., Murphy, W. J. (2008) Neuroendocrine hormones such as growth hormone and prolactin are integral members of the immunological cytokine network. Cellular Immunology 252, 111-121.

142. Sutherland, J. S., Goldberg, G. L., Hammett, M. V., Uldrich, A. P., Berzins, S. P., Heng, T. S., Blazar, B. R., Millar, J. L., Malin, M. A., Chidgey, A. P. (2005) Activation of thymic regeneration in mice and humans following androgen blockade. The Journal of Immunology 175, 2741-2753.

143. Mehr, R. and Melamed, D. (2011) Reversing B cell aging. Aging 3, 438-443.

144. Minet-Quinard, R., Farges, M. C., Thivat, E., Deleine, C., Mayot, G., Brtko, J., Ribalta, J., Winklhofer-Roob, B., Rock, E., Vasson, M. P. (2010) Neutrophils are immune cells preferentially targeted by retinoic acid in elderly. Immunity \& Ageing 7:10. 
145. Xu, Q., Parks, C. G., DeRoo, L. A., Cawthon, R. M., Sandler, D. P., Chen, H. (2009) Multivitamin use and telomere length in women. American Journal of Clinical Nutrition 89, 1857-1863.

146. Cakman, I., Kirchner, H., Rink, L. (1997) Zinc supplementation reconstitutes the production of interferon- by leukocytes from elderly persons. Journal of Interferon \& Cytokine Research 17, 469-472.

147. Mocchegiani, E., Basso, A., Giacconi, R., Piacenza, F., Costarelli, L., Pierpaoli, S., Malavolta, M. (2010) Diet (zinc)-gene interaction related to inflammatory/immune response in ageing: possible link with frailty syndrome? Biogerontology, 1-7.

148. Mocchegiani, E. and Malavolta, M. (2004) NK and NKT cell functions in immunosenescence. Aging Cell 3, 177-184.

149. Chen, D. and Guarente, L. (2007) SIR2: a potential target for calorie restriction mimetics. Trends in Molecular Medicine 13, 64-71.

150. Howitz, K. T., Bitterman, K. J., Cohen, H. Y., Lamming, D. W., Lavu, S., Wood, J. G., Zipkin, R. E., Chung, P., Kisielewski, A., Zhang, L. L. (2003) Small molecule activators of sirtuins extend Saccharomyces cerevisiae lifespan. Nature 425, 191-196.

151. Baur, J. A., Pearson, K. J., Price, N. L., Jamieson, H. A., Lerin, C., Kalra, A., Prabhu, V. V., Allard, J. S., Lopez-Lluch, G., Lewis, K. (2006) Resveratrol improves health and survival of mice on a high-calorie diet. Nature 444, 337342.

152. Sierra, F., Hadley, E., Suzman, R., Hodes, R. (2009) Prospects for Life Span Extension. Annual Review of Medicine 60, 457-469.

153. Yang, H., Youm, Y. H., Dixit, V. D. (2009) Inhibition of thymic adipogenesis by caloric restriction is coupled with reduction in age-related thymic involution. The Journal of Immunology 183, 3040-3052.

154. Colman, R. J., Anderson, R. M., Johnson, S. C., Kastman, E. K., Kosmatka, K. J., Beasley, T. M., Allison, D. B., Cruzen, C., Simmons, H. A., Kemnitz, J. W. (2009) Caloric restriction delays disease onset and mortality in rhesus monkeys. Science 325, 201-204.

155. Messaoudi, I., Warner, J., Fischer, M., Park, B., Hill, B., Mattison, J., Lane, M. A., Roth, G. S., Ingram, D. K., Picker, L. J. (2006) Delay of T cell senescence by caloric restriction in aged long-lived nonhuman primates. Proceedings of the National Academy of Sciences 103, 19448-19453.

156. Ritz, B. W. and Gardner, E. M. (2006) Malnutrition and energy restriction differentially affect viral immunity. The Journal of Nutrition 136, 1141-1144.

157. Ritz, B. W., Aktan, I., Nogusa, S., Gardner, E. M. (2008) Energy restriction impairs natural killer cell function and increases the severity of influenza infection in young adult male C57BL/6 mice. The Journal of Nutrition 138, 2269-2275.

158. Lara-Padilla, E., Campos-Rodríguez, R., Jarillo-Luna, A., Reyna-Garfias, H., Rivera-Aguilar, V., Miliar, A., Berral de la Rosa, F. J., Navas, P., López-Lluch, G. (2011) Caloric restriction reduces IgA levels and modifies cytokine mRNA expression in mouse small intestine. The Journal of Nutritional Biochemistry 22, 560-566.

159. Malaguarnera, L., Cristaldi, E., Lipari, H., Malaguarnera, M. (2008) Acquired immunity: immunosenescence and physical activity. European Review of Aging and Physical Activity 5, 61-68. 
160. FAO/WHO (2001) Evaluation of health and nutritional properties of powder milk and live lactic acid bacteria. Report of a Joint FAO/WHO Expert Consultation, Córdoba, Argentina.

161. Girardin, M. and Seidman, E. G. (2011) Indications for the use of probiotics in gastrointestinal diseases. Digestive Diseases 29, 574-587.

162. Foligne, B., Zoumpopoulou, G., Dewulf, J., Younes, A. B., Chareyre, F., Sirard, J.-C., Pot, B., Grangette, C. (2007) A key role of dendritic cells in probiotic functionality. PLoS ONE 2, e313.

163. Karczewski, J., Troost, F. J., Konings, I., Dekker, J., Kleerebezem, M., Brummer, R.-J. M., Wells, J. M. (2010) Regulation of human epithelial tight junction proteins by Lactobacillus plantarum in vivo and protective effects on the epithelial barrier. American Journal of Physiology-Gastrointestinal and Liver Physiology 298, G851-G859.

164. Sherman, P. M., Ossa, J. C., Johnson-Henry, K. (2009) Unraveling mechanisms of action of probiotics. Nutrition in Clinical Practice 24, 10-14.

165. Matsumoto, M., Kurihara, S., Kibe, R., Ashida, H., Benno, Y. (2011) Longevity in mice is promoted by probiotic-induced suppression of colonic senescence dependent on upregulation of gut bacterial polyamine production. PLoS One 6 , e23652.

166. Kibe, R., Kurihara, S., Sakai, Y., Suzuki, H., Ooga, T., Sawaki, E., Muramatsu, K., Nakamura, A., Yamashita, A., Kitada, Y. (2014) Upregulation of colonic luminal polyamines produced by intestinal microbiota delays senescence in mice. Scientific Reports 4, 4548.

167. Sharma, R., Kapila, R., Kapasiya, M., Saliganti, V., Dass, G., Kapila, S. (2014) Dietary supplementation of milk fermented with probiotic Lactobacillus fermentum enhances systemic immune response and antioxidant capacity in aging mice. Nutrition Research 34, 968-981.

168. Jeong, J.-J., Kim, K.-A., Jang, S.-E., Woo, J.-Y., Han, M. J., Kim, D.-H. (2015) Orally administrated Lactobacillus pentosus var. plantarum C29 ameliorates age-dependent colitis by inhibiting the nuclear factor-kappa B signaling pathway via the regulation of lipopolysaccharide production by gut microbiota. PLoS ONE 10, e0116533.

169. Jeong, J.-J., Woo, J.-Y., Ahn, Y.-T., Shim, J.-H., Huh, C.-S., Im, S.-H., Han, M. J., Kim, D.-H. (2015) The probiotic mixture IRT5 ameliorates agedependent colitis in rats. International Immunopharmacology 26, 416-422.

170. Lahtinen, S. J., Forssten, S., Aakko, J., Granlund, L., Rautonen, N., Salminen, S., Viitanen, M., Ouwehand, A. C. (2011) Probiotic cheese containing Lactobacillus rhamnosus HN001 and Lactobacillus acidophilus NCFM® modifies subpopulations of fecal lactobacilli and Clostridium difficile in the elderly. AGE, 1-11.

171. Candore, G., Balistreri, C. R., Colonna-Romano, G., Grimaldi, M. P., Lio, D., Listi', F., Scola, L., Vasto, S., Caruso, C. (2008) Immunosenescence and antiimmunosenescence therapies: the case of probiotics. Rejuvenation Research $11,425-432$.

172. Maneerat, S., Lehtinen, M. J., Childs, C. E., Forssten, S. D., Alhoniemi, E., Tiphaine, M., Yaqoob, P., Ouwehand, A. C., Rastall, R. A. (2013) Consumption of Bifidobacterium lactis $\mathrm{Bi}-07$ by healthy elderly adults enhances phagocytic activity of monocytes and granulocytes. Journal of Nutritional Science 2, e44. 
173. Miller, R. A., Buehner, G., Chang, Y., Harper, J. M., Sigler, R., SmithWheelock, M. (2005) Methionine-deficient diet extends mouse lifespan, slows immune and lens aging, alters glucose, T4, IGF-I and insulin levels, and increases hepatocyte MIF levels and stress resistance. Aging Cell 4, 119-125.

174. Segall, P. E. and Timiras, P. S. (1976) Patho-physiologic findings after chronic tryptophan deficiency in rats: a model for delayed growth and aging. Mechanisms of Ageing and Development 5, 109-124.

175. Ooka, H., Segall, P. E., Timiras, P. S. (1988) Histology and survival in agedelayed low-tryptophan-fed rats. Mechanisms of Ageing and Development 43, 79-98.

176. de Marte, M. L. and Enesco, H. E. (1986) Influence of low tryptophan diet on survival and organ growth in mice. Mechanisms of Ageing and Development 36, 161-171.

177. Keszthelyi, D., Troost, F., Masclee, A. (2009) Understanding the role of tryptophan and serotonin metabolism in gastrointestinal function. Neurogastroenterology \& Motility 21, 1239-1249.

178. Grohmann, U., Fallarino, F., Puccetti, P. (2003) Tolerance, DCs and tryptophan: much ado about IDO. Trends in Immunology 24, 242-248.

179. Le Floc'h, N., Otten, W., Merlot, E. (2011) Tryptophan metabolism, from nutrition to potential therapeutic applications. Amino Acids 41, 1195-1205.

180. Terness, P., Bauer, T. M., Röse, L., Dufter, C., Watzlik, A., Simon, H., Opelz, G. (2002) Inhibition of Allogeneic T Cell Proliferation by Indoleamine 2, 3Dioxygenase-expressing Dendritic Cells Mediation of Suppression by Tryptophan Metabolites. The Journal of Experimental Medicine 196, 447-457.

181. Frick, B., Schroecksnadel, K., Neurauter, G., Leblhuber, F., Fuchs, D. (2004) Increasing production of homocysteine and neopterin and degradation of tryptophan with older age. Clinical Biochemistry 37, 684-687.

182. Schröcksnadel, K., Wirleitner, B., Winkler, C., Fuchs, D. (2006) Monitoring tryptophan metabolism in chronic immune activation. Clinica Chimica Acta 364, 82-90.

183. Van der Goot, A. T. and Nollen, E. A. (2013) Tryptophan metabolism: entering the field of aging and age-related pathologies. Trends in Molecular Medicine 19, 336-344.

184. Capuron, L., Schroecksnadel, S., Féart, C., Aubert, A., Higueret, D., Barberger-Gateau, P., Layé, S., Fuchs, D. (2011) Chronic low-grade inflammation in elderly persons is associated with altered tryptophan and tyrosine metabolism: role in neuropsychiatric symptoms. Biological Psychiatry 70, 175-182.

185. Smelt, M. J., de Haan, B. J., Bron, P. A., van Swam, I., Meijerink, M., Wells, J. M., Faas, M. M., de Vos, P. (2013) Probiotics can generate FoxP3 T-cell responses in the small intestine and simultaneously inducing CD4 and CD8 T cell activation in the large intestine. PLoS ONE 8, e68952.

186. Smelt, M. J., de Haan, B. J., Bron, P. A., van Swam, I., Meijerink, M., Wells, J. M., Kleerebezem, M., Faas, M. M., de Vos, P. (2013) The impact of Lactobacillus plantarum WCFS1 teichoic acid D-alanylation on the generation of effector and regulatory T-cells in healthy mice. PLoS ONE 8, e63099.

187. Ehrlich, P. (1879) Beitrage zur Kenntniss der granulirten Zellen u.s.w. Verhandlungen der physiol. Gesellschaft Berlin 21, 53. 
188. Ishizaka, T., DeBernardo, R., Tomioka, H., Lichtenstein, L. M., Ishizaka, K. (1972) Identification of basophil granulocytes as a site of allergic histamine release. The Journal of Immunology 108, 1000-1008.

189. Stone, K. D., Prussin, C., Metcalfe, D. D. (2010) lgE, mast cells, basophils, and eosinophils. Journal of Allergy and Clinical Immunology 125, S73-S80.

190. Karasuyama, H. (2011) Nonredundant Roles of Basophils in Immunity. Annual Review of Immunology 29, 45-69.

191. Brunner, T., Heusser, C., Dahinden, C. (1993) Human peripheral blood basophils primed by interleukin 3 (IL-3) produce IL-4 in response to immunoglobulin $\mathrm{E}$ receptor stimulation. The Journal of Experimental Medicine 177, 605-611.

192. Piccinni, M. P., Macchia, D., Parronchi, P., Giudizi, M. G., Bani, D., Alterini, R., Grossi, A., Ricci, M., Maggi, E., Romagnani, S. (1991) Human bone marrow non- $B$, non- $T$ cells produce interleukin 4 in response to cross-linkage of $\mathrm{Fc} \varepsilon$ and $\mathrm{Fc} \gamma$ receptors. Proceedings of the National Academy of Sciences 88, 8656-8660.

193. Schroeder, J. T., MacGlashan, D., Kagey-Sobotka, A., White, J. M., Lichtenstein, L. M. (1994) IgE-dependent IL-4 secretion by human basophils. The relationship between cytokine production and histamine release in mixed leukocyte cultures. The Journal of Immunology 153, 1808-1817.

194. Schroeder, J. T. and MacGlashan, D. W. (2001) Human basophils: mediator release and cytokine production. Advances in Immunology 77, 93-122.

195. Ohmori, K., Luo, Y., Jia, Y., Nishida, J., Wang, Z., Bunting, K. D., Wang, D., Huang, H. (2009) IL-3 induces basophil expansion in vivo by directing granulocyte-monocyte progenitors to differentiate into basophil lineagerestricted progenitors in the bone marrow and by increasing the number of basophil/mast cell progenitors in the spleen. The Journal of Immunology 182, 2835-2841.

196. Yoshimura-Uchiyama, C., Yamaguchi, M., Nagase, H., Fujisawa, T., Ra, C., Matsushima, K., Iwata, T., Igarashi, T., Yamamoto, K., Hirai, K. (2003) Comparative effects of basophil-directed growth factors. Biochemical and Biophysical Research Communications 302, 201-206.

197. Pecaric-Petkovic, T., Didichenko, S. A., Kaempfer, S., Spiegl, N., Dahinden, C. A. (2009) Human basophils and eosinophils are the direct target leukocytes of the novel IL-1 family member IL-33. Blood 113, 1526-1534.

198. Schroeder, J. T. (2011) Basophils: emerging roles in the pathogenesis of allergic disease. Immunological Reviews 242, 144-160.

199. Suzukawa, M., Nagase, H., Ogahara, I., Han, K., Tashimo, H., Shibui, A., Koketsu, R., Nakae, S., Yamaguchi, M., Ohta, K. (2011) Leptin Enhances Survival and Induces Migration, Degranulation, and Cytokine Synthesis of Human Basophils. The Journal of Immunology 186, 5254-5260.

200. Siracusa, M. C., Saenz, S. A., Hill, D. A., Kim, B. S., Headley, M. B., Doering, T. A., Wherry, E. J., Jessup, H. K., Siegel, L. A., Kambayashi, T. (2011) TSLP promotes interleukin-3-independent basophil haematopoiesis and type 2 inflammation. Nature 477, 229-233.

201. Perrigoue, J. G., Saenz, S. A., Siracusa, M. C., Allenspach, E. J., Taylor, B. C., Giacomin, P. R., Nair, M. G., Du, Y., Zaph, C., Van Rooijen, N. (2009) MHC class II-dependent basophil-CD4+ $\mathrm{T}$ cell interactions promote $\mathrm{TH} 2$ cytokine-dependent immunity. Nature Immunology 10, 697-705. 
202. Comeau, M. and Ziegler, S. (2009) The influence of TSLP on the allergic response. Mucosal Immunology 3, 138-147.

203. He, R. and Geha, R. (2010) Thymic stromal lymphopoietin. Annals of the New York Academy of Sciences 1183, 13-24.

204. Liu, Y. J., Soumelis, V., Watanabe, N., Ito, T., Wang, Y. H., de Waal Malefyt, R., Omori, M., Zhou, B., Ziegler, S. F. (2007) TSLP: an epithelial cell cytokine that regulates $\mathrm{T}$ cell differentiation by conditioning dendritic cell maturation. Immunology 25, 193-219.

205. Soumelis, V. and Liu, Y. J. (2004) Human thymic stromal lymphopoietin: a novel epithelial cell-derived cytokine and a potential key player in the induction of allergic inflammation. 25, 325-333.

206. Ziegler, S. and Artis, D. (2010) Sensing the outside world: TSLP regulates barrier immunity. Nature Immunology 11, 289-293.

207. Sloane, D. E. and MacGlashan, D. (2011) Basophils in Anaphylaxis. Anaphylaxis and Hypersensitivity Reactions, 69-87.

208. Schneider, E., Leite-de-Moraes, M., Dy, M. (2011) Histamine, Immune Cells and Autoimmunity. Advances in Experimental Medicine and Biology 709, 8194.

209. Tsujimura, Y., Obata, K., Mukai, K., Shindou, H., Yoshida, M., Nishikado, H., Kawano, Y., Minegishi, Y., Shimizu, T., Karasuyama, H. (2008) Basophils play a pivotal role in immunoglobulin-G-mediated but not immunoglobulin-Emediated systemic anaphylaxis. Immunity 28, 581-589.

210. Finkelman, F. D. (2011) IgE-Dependent and Independent Effector Mechanisms in Human and Murine Anaphylaxis. In Anaphylaxis and Hypersensitivity Reactions (M. C. Castells, ed) Springer, New York 127-144.

211. Klos, A., Tenner, A. J., Johswich, K. O., Ager, R. R., Reis, E. S., Köhl, J. (2009) The role of the anaphylatoxins in health and disease. Molecular immunology 46, 2753-2766.

212. Guo, R. F. and Ward, P. A. (2005) Role of C5a in inflammatory responses. Annu. Rev. Immunol. 23, 821-852.

213. Schneider, E., Thieblemont, N., De Moraes, M. L., Dy, M. (2010) Basophils: new players in the cytokine network. European Cytokine Network 21, 142153.

214. Gessner, A., Mohrs, K., Mohrs, M. (2005) Mast cells, basophils, and eosinophils acquire constitutive IL-4 and IL-13 transcripts during lineage differentiation that are sufficient for rapid cytokine production. The Journal of Immunology 174, 1063-1072.

215. Ohnmacht, C. and Voehringer, D. (2009) Basophil effector function and homeostasis during helminth infection. Blood 113, 2816-2825.

216. Clarke, E. K., Xenakis, J. J., Spencer, L. A. (2011) Differential Expression of Pre-formed Cytokines in Human Basophils and Eosinophils. J Allergy Clin Immunol 127, AB208.

217. Humphreys, N. E., Xu, D., Hepworth, M. R., Liew, F. Y., Grencis, R. K. (2008) IL-33, a potent inducer of adaptive immunity to intestinal nematodes. The Journal of Immunology 180, 2443-2449.

218. Liew, F. Y., Pitman, N. I., Mclnnes, I. B. (2010) Disease-associated functions of IL-33: the new kid in the IL-1 family. Nature Reviews Immunology 10, 103110.

219. Lloyd, C. M. (2010) IL-33 family members and asthma-bridging innate and adaptive immune responses. Current Opinion in Immunology 22, 800-806. 
220. Suzukawa, M., likura, M., Koketsu, R., Nagase, H., Tamura, C., Komiya, A., Nakae, S., Matsushima, K., Ohta, K., Yamamoto, K. (2008) An IL-1 cytokine member, IL-33, induces human basophil activation via its ST2 receptor. The Journal of Immunology 181, 5981-5989.

221. Blom, L., Poulsen, B. C., Jensen, B. M., Hansen, A., Poulsen, L. K. (2011) IL33 Induces IL-9 Production in Human CD4+ T Cells and Basophils. PLoS One 6, e21695.

222. Sokol, C. L., Barton, G. M., Farr, A. G., Medzhitov, R. (2008) A mechanism for the initiation of allergen-induced $T$ helper type 2 responses. Nature Immunology 9, 310-318.

223. Wang, Y. H., Ito, T., Homey, B., Watanabe, N., Martin, R., Barnes, C. J., McIntyre, B. W., Gilliet, M., Kumar, R. (2006) Maintenance and polarization of human $\mathrm{TH} 2$ central memory $\mathrm{T}$ cells by thymic stromal lymphopoietin-activated dendritic cells. Immunity 24, 827-838.

224. Wang, J. and Xing, F. (2008) Human TSLP-Educated DCs. Cellular and Molecular Immunology 5, 99-106.

225. Wang, Y. and Liu, Y. (2009) TSLP, OX40L, and IL-25 in Allergic Responses. Clinical and experimental allergy: journal of the British Society for Allergy and Clinical Immunology 39, 798-806.

226. Wang, Y. H., Angkasekwinai, P., Lu, N., Voo, K. S., Arima, K., Hanabuchi, S., Hippe, A., Corrigan, C. J., Dong, C., Homey, B. (2007) IL-25 augments type 2 immune responses by enhancing the expansion and functions of TSLP-DCactivated Th2 memory cells. The Journal of Experimental Medicine 204, 18371847.

227. Spiegl, N., Didichenko, S., McCaffery, P., Langen, H., Dahinden, C. A. (2008) Human basophils activated by mast cell-derived IL-3 express retinaldehyde dehydrogenase-II and produce the immunoregulatory mediator retinoic acid. Blood 112, 3762-3771.

228. Boniface, K., Blom, B., Liu, Y. J., de Waal Malefyt, R. (2008) From interleukin23 to T-helper 17 cells: human T-helper cell differentiation revisited. Immunlogical Reviews 226, 132-146.

229. Klemann, C., Je Raveney, B., Oki, S., Yamamura, T. (2009) Retinoid signals and Th17-mediated pathology. Japanese Journal of Clinical Immunology 32, 20-28.

230. Qi, Y., Operario, D. J., Oberholzer, C. M., Kobie, J. J., Looney, R. J., Georas, S. N., Mosmann, T. R. (2010) Human basophils express amphiregulin in response to T cell-derived IL-3. Journal of Allergy and Clinical Immunology 126, $1260-1266$.

231. Zaiss, D. M., Yang, L., Shah, P. R., Kobie, J. J., Urban, J. F., Mosmann, T. R. (2006) Amphiregulin, a TH2 cytokine enhancing resistance to nematodes. Science 314, 1746.

232. De Paulis, A., Prevete, N., Fiorentino, I., Rossi, F. W., Staibano, S., Montuori, N., Ragno, P., Longobardi, A., Liccardo, B., Genovese, A. (2006) Expression and functions of the vascular endothelial growth factors and their receptors in human basophils. The Journal of Immunology 177, 7322-7331.

233. Ahdieh, M., Vandenbos, T., Youakim, A. (2001) Lung epithelial barrier function and wound healing are decreased by IL-4 and IL-13 and enhanced by IFN- $\gamma$. American Journal of Physiology-Cell Physiology 281, C2029-C2038. 
234. Ito, Y., Satoh, T., Takayama, K., Miyagishi, C., Walls, A., Yokozeki, H. (2011) Basophil recruitment and activation in inflammatory skin diseases. Allergy 66, 1107-1113.

235. Naclerio, R. M., Proud, D., Togias, A. G., Adkinson Jr, N. F., Meyers, D. A., Kagey-Sobotka, A., Plaut, M., Norman, P. S., Lichtenstein, L. M. (1985) Inflammatory mediators in late antigen-induced rhinitis. New England Journal of Medicine 313, 65-70.

236. Falcone, F. H., Haas, H., Gibbs, B. F. (2000) The human basophil: a new appreciation of its role in immune responses. Blood 96, 4028-4038.

237. Falcone, F., Knol, E., Gibbs, B. (2011) The role of basophils in the pathogenesis of allergic disease. Clinical \& Experimental Allergy 41, 939-947.

238. Knol, E. F. and Gibbs, B. F. (2009) Editorial: Basophil survival and immunomodulatory function are uniquely regulated by a novel MyD88dependent pathway. Journal of Leukocyte Biology 86, 753-755.

239. Kroeger, K. M., Sullivan, B. M., Locksley, R. M. (2009) IL-18 and IL-33 elicit Th2 cytokines from basophils via a MyD88-and p38a-dependent pathway. Journal of Leukocyte Biology 86, 769-778.

240. van Panhuys, N., Prout, M., Forbes, E., Min, B., Paul, W. E., Le Gros, G. (2011) Basophils are the major producers of IL-4 during primary helminth infection. The Journal of Immunology 186, 2719-2728.

241. Voehringer, D., Shinkai, K., Locksley, R. M. (2004) Type 2 immunity reflects orchestrated recruitment of cells committed to IL-4 production. Immunity 20, 267-277.

242. Chen, K., Xu, W., Wilson, M., He, B., Miller, N. W., Bengtén, E., Edholm, E. S., Santini, P. A., Rath, P., Chiu, A. (2009) Immunoglobulin D enhances immune surveillance by activating antimicrobial, proinflammatory and B cellstimulating programs in basophils. Nature Immunology 10, 889-898.

243. Gomez, M. R., Talke, Y., Goebel, N., Hermann, F., Reich, B., Mack, M. (2010) Basophils support the survival of plasma cells in mice. The Journal of Immunology 185, 7180-7185.

244. Bosch, X., Lozano, F., Cervera, R., Ramos-Casals, M., Min, B. (2011) Basophils, IgE, and Autoantibody-Mediated Kidney Disease. The Journal of Immunology 186, 6083-6090.

245. Schuerwegh, A., loan-Facsinay, A., Dorjée, A., Roos, J., Bajema, I., van der Voort, E., Huizinga, T., Toes, R. (2010) Evidence for a functional role of lgE anticitrullinated protein antibodies in rheumatoid arthritis. Proceedings of the National Academy of Sciences 107, 2586-2591.

246. Kaveri, S. V., Mouthon, L., Bayry, J. (2010) Basophils and nephritis in lupus. New England Journal of Medicine 363, 1080-1082.

247. Charles, N., Hardwick, D., Daugas, E., Illei, G. G., Rivera, J. (2010) Basophils and the $\mathrm{T}$ helper 2 environment can promote the development of lupus nephritis. Nature Medicine 16, 701-707.

248. Nakanishi, K. (2010) Basophils as APC in Th2 response in allergic inflammation and parasite infection. Current Opinion in Immunology 22, 814820.

249. Smith, D. (2010) IL 33: a tissue derived cytokine pathway involved in allergic inflammation and asthma. Clinical \& Experimental Allergy 40, 200-208.

250. Phillips, C., Coward, W. R., Pritchard, D. I., Hewitt, C. R. A. (2003) Basophils express a type 2 cytokine profile on exposure to proteases from helminths and house dust mites. Journal of Leukocyte Biology 73, 165-171. 
251. Banchereau, J. and Steinman, R. M. (1998) Dendritic cells and the control of immunity. Nature 392, 245-252.

252. Sokol, C. L., Chu, N. Q., Yu, S., Nish, S. A., Laufer, T. M., Medzhitov, R. (2009) Basophils function as antigen-presenting cells for an allergen-induced T helper type 2 response. Nature Immunology 10, 713-720.

253. Yoshimoto, T., Yasuda, K., Tanaka, H., Nakahira, M., Imai, Y., Fujimori, Y., Nakanishi, K. (2009) Basophils contribute to TH2-IgE responses in vivo via IL4 production and presentation of peptide-MHC class II complexes to CD4+ T cells. Nature Immunology 10, 706-712.

254. Hammad, H., Plantinga, M., Deswarte, K., Pouliot, P., Willart, M. A. M., Kool, M., Muskens, F., Lambrecht, B. N. (2010) Inflammatory dendritic cells-not basophils-are necessary and sufficient for induction of Th2 immunity to inhaled house dust mite allergen. The Journal of Experimental Medicine 207, 2097-2111.

255. Ohnmacht, C. and Voehringer, D. (2010) Basophils protect against reinfection with hookworms independently of mast cells and memory Th2 cells. The Journal of Immunology 184, 344-350.

256. Phythian-Adams, A. T., Cook, P. C., Lundie, R. J., Jones, L. H., Smith, K. A., Barr, T. A., Hochweller, K., Anderton, S. M., Hämmerling, G. J., Maizels, R. M. (2010) CD11c depletion severely disrupts Th2 induction and development in vivo. The Journal of Experimental Medicine 207, 2089-2096.

257. Tang, H., Cao, W., Kasturi, S. P., Ravindran, R., Nakaya, H. I., Kundu, K., Murthy, N., Kepler, T. B., Malissen, B., Pulendran, B. (2010) The T helper type 2 response to cysteine proteases requires dendritic cell-basophil cooperation via ROS-mediated signaling. Nature Immunology 11, 608-617.

258. Ohnmacht, C., Schwartz, C., Panzer, M., Schiedewitz, I., Naumann, R., Voehringer, D. (2010) Basophils orchestrate chronic allergic dermatitis and protective immunity against helminths. Immunity 33, 364-374.

259. Kim, S., Prout, M., Ramshaw, H., Lopez, A. F., LeGros, G., Min, B. (2010) Cutting edge: basophils are transiently recruited into the draining lymph nodes during helminth infection via IL-3, but infection-induced Th2 immunity can develop without basophil lymph node recruitment or IL-3. The Journal of Immunology 184, 1143-1147.

260. Sullivan, B. M., Liang, H. E., Bando, J. K., Wu, D., Cheng, L. E., McKerrow, J. K., Allen, C. D. C., Locksley, R. M. (2011) Genetic analysis of basophil function in vivo. Nature Immunology 12, 527-535.

261. Mukai, K., Matsuoka, K., Taya, C., Suzuki, H., Yokozeki, H., Nishioka, K., Hirokawa, K., Etori, M., Yamashita, M., Kubota, T. (2005) Basophils play a critical role in the development of IgE-mediated chronic allergic inflammation independently of T cells and mast cells. Immunity 23, 191-202.

262. Mikhak, Z. and Luster, A. D. (2007) Chemokines in allergic responses: eosinophils, basophils, mast cells. In Chemokine Biology-Basic Research and Clinical Application, Volume II (K. Neote, G. L. Letts, and B. Moser, eds), Birkhäuser Verlag, Basel 27-41.

263. Kepley, C. L., Cambier, J. C., Morel, P. A., Lujan, D., Ortega, E., Wilson, B. S., Oliver, J. M. (2000) Negative regulation of FceRI signaling by FcgRII costimulation in human blood basophils. Journal of Allergy and Clinical Immunology 106, 337-348.

264. Meknache, N., Jönsson, F., Laurent, J., Guinnepain, M. T., Daëron, M. (2009) Human Basophils Express the Glycosylphosphatidylinositol-Anchored Low- 
Affinity IgG Receptor FcyRIIIB (CD16B). The Journal of Immunology 182, 2542-2550.

265. Malbec, O. and Daëron, M. (2007) The mast cell IgG receptors and their roles in tissue inflammation. Immunological reviews 217, 206-221.

266. Khodoun, M. V., Strait, R., Armstrong, L., Yanase, N., Finkelman, F. D. (2011) Identification of markers that distinguish IgE-from IgG-mediated anaphylaxis. Proceedings of the National Academy of Sciences 108, 12413-12418.

267. Vadas, P., Gold, M., Perelman, B., Liss, G. M., Lack, G., Blyth, T., Simons, F. E. R., Simons, K. J., Cass, D., Yeung, J. (2008) Platelet-activating factor, PAF acetylhydrolase, and severe anaphylaxis. New England Journal of Medicine 358, 28-35.

268. Kitzmüller, C., Nagl, B., Deifl, S., Walterskirchen, C., Jahn-Schmid, B., Zlabinger, G., Bohle, B. (2012) Human blood basophils do not act as antigenpresenting cells for the major birch pollen allergen Bet $v 1$. Allergy $67,593-$ 600.

269. Eckl-Dorna, J., Ellinger, A., Blatt, K., Ghanim, V., Steiner, I., Pavelka, M., Valent, P., Valenta, R., Niederberger, V. (2012) Basophils are not the key antigen-presenting cells in allergic patients. Allergy 67, 601-608.

270. Poulsen, B. C., Poulsen, L. K., Jensen, B. M. (2012) Detection of MHC class II expression on human basophils is dependent on antibody specificity but independent of atopic disposition. Journal of Immunological Methods 381, 6669.

271. Voskamp, A. L., Prickett, S. R., Mackay, F., Rolland, J. M., O'Hehir, R. E. (2013) MHC class II expression in human basophils: induction and lack of functional significance. PLoS ONE 8, e81777.

272. Yoshimoto, T. and Nakanishi, K. (2012) Generation and Characterization of Mouse Basophils from Bone Marrow and Purification of Basophils from Spleen. Current Protocols in Immunology, 3.24.1-3.24.16.

273. Kondo, Y., Yoshimoto, T., Yasuda, K., Futatsugi-Yumikura, S., Morimoto, M., Hayashi, N., Hoshino, T., Fujimoto, J., Nakanishi, K. (2008) Administration of IL-33 induces airway hyperresponsiveness and goblet cell hyperplasia in the lungs in the absence of adaptive immune system. International Immunology 20, 791-800.

274. Lutz, M. B., Suri, R. M., Niimi, M., Ogilvie, A. L., Kukutsch, N. A., Rößner, S., Schuler, G., Austyn, J. M. (2000) Immature dendritic cells generated with low doses of GM-CSF in the absence of IL-4 are maturation resistant and prolong allograft survival in vivo. European Journal of Immunology 30, 1813-1822.

275. Liu, Y.-J., Soumelis, V., Watanabe, N., Ito, T., Wang, Y.-H., de Waal Malefyt, R., Omori, M., Zhou, B., Ziegler, S. F. (2007) TSLP: an epithelial cell cytokine that regulates $\mathrm{T}$ cell differentiation by conditioning dendritic cell maturation. Annual Reviews in Immunology 25, 193-219.

276. Wang, J. and Xing, F. (2008) Human TSLP-educated DCs. Cell Mol Immunol 5, 99-106.

277. Wang, Y. H. and Liu, Y. J. (2009) Thymic stromal lymphopoietin, OX40-ligand, and interleukin-25 in allergic responses. Clinical \& Experimental Allergy 39, 798-806.

278. Zhou, B., Comeau, M. R., Thibaut De Smedt, H., Dahl, M. E., Lewis, D. B., Gyarmati, D., Aye, T., Campbell, D. J., Ziegler, S. F. (2005) Thymic stromal lymphopoietin as a key initiator of allergic airway inflammation in mice. Nature Immunology 6, 1047-1053. 
279. Luo, Y., Chen, G.-L., Hannemann, N., Ipseiz, N., Krönke, G., Bäuerle, T., Munos, L., Wirtz, S., Schett, G., Bozec, A. (2015) Microbiota from Obese Mice Regulate Hematopoietic Stem Cell Differentiation by Altering the Bone Niche. Cell Metabolism 22, 886-894.

280. Clarke, T. B., Davis, K. M., Lysenko, E. S., Zhou, A. Y., Yu, Y., Weiser, J. N. (2010) Recognition of peptidoglycan from the microbiota by Nod1 enhances systemic innate immunity. Nature Medicine 16, 228-231.

281. Hill, D. A., Siracusa, M. C., Abt, M. C., Kim, B. S., Kobuley, D., Kubo, M., Kambayashi, T., LaRosa, D. F., Renner, E. D., Orange, J. S. (2012) Commensal bacteria-derived signals regulate basophil hematopoiesis and allergic inflammation. Nature Medicine 18, 538-546.

282. Smith, P., Dunne, D. W., Fallon, P. G. (2001) Defective in vivo induction of functional type 2 cytokine responses in aged mice. European Journal of Immunology 31, 1495-1502.

283. Uciechowski, P. and Rink, L. (2014) Basophil, Eosinophil, and Neutrophil Functions in the Elderly. In Immunology of AgingSpringer 47-63.

284. Schwarzenbach, H., Nakagawa, T., Conroy, M., WECK, A. (1982) Skin reactivity, basophil degranulation and $\lg \mathrm{E}$ levels in ageing. Clinical \& Experimental Allergy 12, 465-473.

285. Leng, S. X., Xue, Q.-L., Huang, Y., Ferrucci, L., Fried, L. P., Walston, J. D. (2005) Baseline total and specific differential white blood cell counts and 5year all-cause mortality in community-dwelling older women. Experimental Gerontology 40, 982-987.

286. Leng, S. X., Xue, Q.-L., Tian, J., Huang, Y., Yeh, S.-H., Fried, L. P. (2009) Associations of neutrophil and monocyte counts with frailty in communitydwelling disabled older women: results from the Women's Health and Aging Studies I. Experimental Gerontology 44, 511-516.

287. Shad, K. F., Aghazadeh, Y., Ahmad, S., Kress, B. (2013) Peripheral markers of Alzheimer's disease: surveillance of white blood cells. Synapse 67, 541543.

288. Song, C., Vandewoude, M., Stevens, W., De Clerck, L., Van der Planken, M., Whelan, A., Anisman, H., Dossche, A., Maes, M. (1999) Alterations in immune functions during normal aging and Alzheimer's disease. Psychiatry Research 85, 71-80.

289. Van Beek, A. A., Knol, E. F., De Vos, P., Smelt, M. J., Savelkoul, H. F. J., Van Neerven, R. J. J. (2013) Recent developments in basophil research: do basophils initiate and perpetuate type 2 T-helper cell responses? International Archives of Allergy and Immunology 160, 7-17.

290. Moore, M. L., Newcomb, D. C., Parekh, V. V., Van Kaer, L., Collins, R. D., Zhou, W., Goleniewska, K., Chi, M. H., Mitchell, D., Boyce, J. A. (2009) STAT1 negatively regulates lung basophil IL-4 expression induced by respiratory syncytial virus infection. The Journal of Immunology 183, 20162026.

291. Denzel, A., Maus, U. A., Gomez, M. R., Moll, C., Niedermeier, M., Winter, C., Maus, R., Hollingshead, S., Briles, D. E., Kunz-Schughart, L. A. (2008) Basophils enhance immunological memory responses. Nature Immunology 9, 733-742.

292. Liang, P., Tang, Y., Fu, S., Lv, J., Liu, B., Feng, M., Li, J., Lai, D., Wan, X., Xu, A. (2015) Basophil count, a marker for disease activity in systemic lupus erythematosus. Clinical Rheumatology 34, 891-896. 
293. Siracusa, M. C., Saenz, S. A., Hill, D. A., Kim, B. S., Headley, M. B., Doering, T. A., Wherry, E. J., Jessup, H. K., Siegel, L. A., Kambayashi, T. (2011) TSLP promotes interleukin-3-independent basophil haematopoiesis and type 2 inflammation. Nature 477, 229-233.

294. Kojima, T., Obata, K., Mukai, K., Sato, S., Takai, T., Minegishi, Y., Karasuyama, H. (2007) Mast cells and basophils are selectively activated in vitro and in vivo through CD200R3 in an IgE-independent manner. The Journal of Immunology 179, 7093-7100.

295. Gerdes, J., Lemke, H., Baisch, H., Wacker, H.-H., Schwab, U., Stein, H. (1984) Cell cycle analysis of a cell proliferation-associated human nuclear antigen defined by the monoclonal antibody Ki-67. The Journal of Immunology 133, 1710-1715.

296. Shiratori, I., Yamaguchi, M., Suzukawa, M., Yamamoto, K., Lanier, L. L., Saito, T., Arase, H. (2005) Down-regulation of basophil function by human CD200 and human herpesvirus-8 CD200. The Journal of Immunology 175, 4441-4449.

297. Lopez-Otin, C., Blasco, M. A., Partridge, L., Serrano, M., Kroemer, G. (2013) The hallmarks of aging. Cell 153, 1194-217.

298. Cuervo, A. M. and Macian, F. (2014) Autophagy and the immune function in aging. Curr Opin Immunol 29, 97-104.

299. Franceschi, C. (2007) Inflammaging as a major characteristic of old people: can it be prevented or cured? Nutr Rev 65, S173-6.

300. Lavin, Y., Mortha, A., Rahman, A., Merad, M. (2015) Regulation of macrophage development and function in peripheral tissues. Nat Rev Immunol 15, 731-44.

301. Biswas, S. K. and Mantovani, A. (2012) Orchestration of metabolism by macrophages. Cell Metab 15, 432-7.

302. Mantovani, A., Biswas, S. K., Galdiero, M. R., Sica, A., Locati, M. (2013) Macrophage plasticity and polarization in tissue repair and remodelling. The Journal of pathology 229, 176-85.

303. Vogelpoel, L. T., Hansen, I. S., Rispens, T., Muller, F. J., van Capel, T. M., Turina, M. C., Vos, J. B., Baeten, D. L., Kapsenberg, M. L., de Jong, E. C., den Dunnen, J. (2014) Fc gamma receptor-TLR cross-talk elicits proinflammatory cytokine production by human M2 macrophages. Nat Commun $5,5444$.

304. Chang, C. P., Su, Y. C., Hu, C. W., Lei, H. Y. (2013) TLR2-dependent selective autophagy regulates NF-kappaB lysosomal degradation in hepatoma-derived M2 macrophage differentiation. Cell Death Differ 20, 51523.

305. Galvan-Pena, S. and O'Neill, L. A. (2014) Metabolic reprograming in macrophage polarization. Front Immunol 5, 420.

306. Tan, Z., Xie, N., Cui, H., Moellering, D. R., Abraham, E., Thannickal, V. J., Liu, G. (2015) Pyruvate dehydrogenase kinase 1 participates in macrophage polarization via regulating glucose metabolism. J Immunol 194, 6082-9.

307. Lavin, Y., Winter, D., Blecher-Gonen, R., David, E., Keren-Shaul, H., Merad, M., Jung, S., Amit, I. (2014) Tissue-resident macrophage enhancer landscapes are shaped by the local microenvironment. Cell 159, 1312-26.

308. Satpathy, A. T., Wu, X., Albring, J. C., Murphy, K. M. (2012) Re(de)fining the dendritic cell lineage. Nat Immunol 13, 1145-54.

309. Lumeng, C. N., Liu, J., Geletka, L., Delaney, C., Delproposto, J., Desai, A., Oatmen, K., Martinez-Santibanez, G., Julius, A., Garg, S., Yung, R. L. (2011) 
Aging is associated with an increase in T cells and inflammatory macrophages in visceral adipose tissue. J Immunol 187, 6208-16.

310. Wang, Y., Wehling-Henricks, M., Samengo, G., Tidball, J. G. (2015) Increases of M2a macrophages and fibrosis in aging muscle are influenced by bone marrow aging and negatively regulated by muscle-derived nitric oxide. Aging Cell 14, 678-88.

311. Natrajan, M. S., de la Fuente, A. G., Crawford, A. H., Linehan, E., Nunez, V., Johnson, K. R., Wu, T., Fitzgerald, D. C., Ricote, M., Bielekova, B., Franklin, R. J. (2015) Retinoid $X$ receptor activation reverses age-related deficiencies in myelin debris phagocytosis and remyelination. Brain 138, 3581-97.

312. Linehan, E., Dombrowski, Y., Snoddy, R., Fallon, P. G., Kissenpfennig, A., Fitzgerald, D. C. (2014) Aging impairs peritoneal but not bone marrow-derived macrophage phagocytosis. Aging Cell 13, 699-708.

313. Mahbub, S., Deburghgraeve, C. R., Kovacs, E. J. (2012) Advanced age impairs macrophage polarization. J Interferon Cytokine Res 32, 18-26.

314. Fei, F., Lee, K. M., McCarry, B. E., Bowdish, D. M. (2016) Age-associated metabolic dysregulation in bone marrow-derived macrophages stimulated with lipopolysaccharide. Sci Rep 6, 22637.

315. Boehmer, E. D., Meehan, M. J., Cutro, B. T., Kovacs, E. J. (2005) Aging negatively skews macrophage TLR2- and TLR4-mediated pro-inflammatory responses without affecting the IL-2-stimulated pathway. Mech Ageing Dev 126, 1305-13.

316. Boyd, A. R., Shivshankar, P., Jiang, S., Berton, M. T., Orihuela, C. J. (2012) Age-related defects in TLR2 signaling diminish the cytokine response by alveolar macrophages during murine pneumococcal pneumonia. Exp Gerontol 47, 507-18.

317. Kollmann, T. R., Levy, O., Montgomery, R. R., Goriely, S. (2012) Innate immune function by Toll-like receptors: distinct responses in newborns and the elderly. Immunity 37, 771-83.

318. Peters, T., Weiss, J. M., Sindrilaru, A., Wang, H., Oreshkova, T., Wlaschek, M., Maity, P., Reimann, J., Scharffetter-Kochanek, K. (2009) Reactive oxygen intermediate-induced pathomechanisms contribute to immunosenescence, chronic inflammation and autoimmunity. Mech Ageing Dev 130, 564-87.

319. Agius, E., Lacy, K. E., Vukmanovic-Stejic, M., Jagger, A. L., Papageorgiou, A. P., Hall, S., Reed, J. R., Curnow, S. J., Fuentes-Duculan, J., Buckley, C. D., Salmon, M., Taams, L. S., Krueger, J., Greenwood, J., Klein, N., Rustin, M. H., Akbar, A. N. (2009) Decreased TNF-alpha synthesis by macrophages restricts cutaneous immunosurveillance by memory CD4+ $\mathrm{T}$ cells during aging. J Exp Med 206, 1929-40.

320. Verschoor, C. P., Johnstone, J., Loeb, M., Bramson, J. L., Bowdish, D. M. (2014) Anti-pneumococcal deficits of monocyte-derived macrophages from the advanced-age, frail elderly and related impairments in PI3K-AKT signaling. Hum Immunol 75, 1192-6.

321. Van Beek, A. A., Hoogerland, J. A., Belzer, C., De Vos, P., De Vos, W. M., Savelkoul, H. F., Leenen, P. J. (2016) Interaction of mouse splenocytes and macrophages with bacterial strains in vitro: the effect of age in the immune response. Benef Microbes 7, 275-87.

322. Guerra-Laso, J. M., Gonzalez-Garcia, S., Gonzalez-Cortes, C., Diez-Tascon, C., Lopez-Medrano, R., Rivero-Lezcano, O. M. (2013) Macrophages from elders are more permissive to intracellular multiplication of Mycobacterium tuberculosis. Age (Dordr) 35, 1235-50. 
323. Henry, C. J., Huang, Y., Wynne, A. M., Godbout, J. P. (2009) Peripheral lipopolysaccharide (LPS) challenge promotes microglial hyperactivity in aged mice that is associated with exaggerated induction of both pro-inflammatory IL-1beta and anti-inflammatory IL-10 cytokines. Brain Behav Immun 23, 30917.

324. Chelvarajan, R. L., Liu, Y., Popa, D., Getchell, M. L., Getchell, T. V., Stromberg, A. J., Bondada, S. (2006) Molecular basis of age-associated cytokine dysregulation in LPS-stimulated macrophages. J Leukoc Biol 79, 1314-27.

325. Wu, D., Marko, M., Claycombe, K., Paulson, K. E., Meydani, S. N. (2003) Ceramide-induced and age-associated increase in macrophage COX-2 expression is mediated through up-regulation of NF-kappa B activity. J Biol Chem 278, 10983-92.

326. Stout, R. D. and Suttles, J. (2005) Immunosenescence and macrophage functional plasticity: dysregulation of macrophage function by age-associated microenvironmental changes. Immunol Rev 205, 60-71.

327. Adolph, T. E., Tomczak, M. F., Niederreiter, L., Ko, H. J., Bock, J., MartinezNaves, E., Glickman, J. N., Tschurtschenthaler, M., Hartwig, J., Hosomi, S., Flak, M. B., Cusick, J. L., Kohno, K., Iwawaki, T., Billmann-Born, S., Raine, T., Bharti, R., Lucius, R., Kweon, M. N., Marciniak, S. J., Choi, A., Hagen, S. J., Schreiber, S., Rosenstiel, P., Kaser, A., Blumberg, R. S. (2013) Paneth cells as a site of origin for intestinal inflammation. Nature 503, 272-6.

328. Rabinowitz, J. D. and White, E. (2010) Autophagy and metabolism. Science 330, 1344-8.

329. Kroemer, G. (2015) Autophagy: a druggable process that is deregulated in aging and human disease. J Clin Invest 125, 1-4.

330. Saitoh, T., Fujita, N., Jang, M. H., Uematsu, S., Yang, B. G., Satoh, T., Omori, H., Noda, T., Yamamoto, N., Komatsu, M., Tanaka, K., Kawai, T., Tsujimura, T., Takeuchi, O., Yoshimori, T., Akira, S. (2008) Loss of the autophagy protein Atg16L1 enhances endotoxin-induced IL-1beta production. Nature 456, 264-8.

331. Harris, J., Hartman, M., Roche, C., Zeng, S. G., O'Shea, A., Sharp, F. A., Lambe, E. M., Creagh, E. M., Golenbock, D. T., Tschopp, J., Kornfeld, H., Fitzgerald, K. A., Lavelle, E. C. (2011) Autophagy controls IL-1beta secretion by targeting pro-IL-1beta for degradation. J Biol Chem 286, 9587-97.

332. Castillo, E. F., Dekonenko, A., Arko-Mensah, J., Mandell, M. A., Dupont, N., Jiang, S., Delgado-Vargas, M., Timmins, G. S., Bhattacharya, D., Yang, H., Hutt, J., Lyons, C. R., Dobos, K. M., Deretic, V. (2012) Autophagy protects against active tuberculosis by suppressing bacterial burden and inflammation. Proc Natl Acad Sci U S A 109, E3168-76.

333. Fattah, E. A., Bhattacharya, A., Herron, A., Safdar, Z., Eissa, N. T. (2015) Critical role for IL-18 in spontaneous lung inflammation caused by autophagy deficiency. J Immunol 194, 5407-16.

334. Zhang, Z., Lowry, S. F., Guarente, L., Haimovich, B. (2010) Roles of SIRT1 in the acute and restorative phases following induction of inflammation. J Biol Chem 285, 41391-401.

335. Roca, H., Varsos, Z. S., Sud, S., Craig, M. J., Ying, C., Pienta, K. J. (2009) CCL2 and interleukin-6 promote survival of human CD11b+ peripheral blood mononuclear cells and induce M2-type macrophage polarization. J Biol Chem 284, 34342-54.

336. Mauer, J., Chaurasia, B., Goldau, J., Vogt, M. C., Ruud, J., Nguyen, K. D., Theurich, S., Hausen, A. C., Schmitz, J., Bronneke, H. S., Estevez, E., Allen, 
T. L., Mesaros, A., Partridge, L., Febbraio, M. A., Chawla, A., Wunderlich, F. T., Bruning, J. C. (2014) Signaling by IL-6 promotes alternative activation of macrophages to limit endotoxemia and obesity-associated resistance to insulin. Nat Immunol 15, 423-30.

337. Sierra-Filardi, E., Nieto, C., Dominguez-Soto, A., Barroso, R., SanchezMateos, P., Puig-Kroger, A., Lopez-Bravo, M., Joven, J., Ardavin, C., Rodriguez-Fernandez, J. L., Sanchez-Torres, C., Mellado, M., Corbi, A. L. (2014) CCL2 shapes macrophage polarization by GM-CSF and M-CSF: identification of CCL2/CCR2-dependent gene expression profile. J Immunol 192, 3858-67.

338. Flotte, T. J., Springer, T. A., Thorbecke, G. J. (1983) Dendritic cell and macrophage staining by monoclonal antibodies in tissue sections and epidermal sheets. Am J Pathol 111, 112-24.

339. Biswas, S. K. and Mantovani, A. (2010) Macrophage plasticity and interaction with lymphocyte subsets: cancer as a paradigm. Nat Immunol 11, 889-96.

340. Loftus, R. M. and Finlay, D. K. (2015) Immunometabolism; cellular metabolism turns immune regulator. $\mathrm{J}$ Biol Chem in press.

341. Pearce, E. L. and Pearce, E. J. (2013) Metabolic pathways in immune cell activation and quiescence. Immunity 38, 633-43.

342. Cramer, T., Yamanishi, Y., Clausen, B. E., Forster, I., Pawlinski, R., Mackman, N., Haase, V. H., Jaenisch, R., Corr, M., Nizet, V., Firestein, G. S., Gerber, H. P., Ferrara, N., Johnson, R. S. (2003) HIF-1alpha is essential for myeloid cell-mediated inflammation. Cell 112, 645-57.

343. Tan, Z., Xie, N., Banerjee, S., Cui, H., Fu, M., Thannickal, V. J., Liu, G. (2015) The monocarboxylate transporter 4 is required for glycolytic reprogramming and inflammatory response in macrophages. J Biol Chem 290, 46-55.

344. Tannahill, G. M., Curtis, A. M., Adamik, J., Palsson-McDermott, E. M., McGettrick, A. F., Goel, G., Frezza, C., Bernard, N. J., Kelly, B., Foley, N. H., Zheng, L., Gardet, A., Tong, Z., Jany, S. S., Corr, S. C., Haneklaus, M., Caffrey, B. E., Pierce, K., Walmsley, S., Beasley, F. C., Cummins, E., Nizet, V., Whyte, M., Taylor, C. T., Lin, H., Masters, S. L., Gottlieb, E., Kelly, V. P., Clish, C., Auron, P. E., Xavier, R. J., O'Neill, L. A. (2013) Succinate is an inflammatory signal that induces IL-1beta through HIF-1alpha. Nature 496, 238-42.

345. Huang, S. C., Everts, B., Ivanova, Y., O'Sullivan, D., Nascimento, M., Smith, A. M., Beatty, W., Love-Gregory, L., Lam, W. Y., O'Neill, C. M., Yan, C., Du, H., Abumrad, N. A., Urban, J. F., Jr., Artyomov, M. N., Pearce, E. L., Pearce, E. J. (2014) Cell-intrinsic lysosomal lipolysis is essential for alternative activation of macrophages. Nat Immunol 15, 846-55.

346. Vats, D., Mukundan, L., Odegaard, J. I., Zhang, L., Smith, K. L., Morel, C. R., Wagner, R. A., Greaves, D. R., Murray, P. J., Chawla, A. (2006) Oxidative metabolism and PGC-1beta attenuate macrophage-mediated inflammation. Cell Metab 4, 13-24.

347. Knuever, J., Willenborg, S., Ding, X., Akyuz, M. D., Partridge, L., Niessen, C. M., Bruning, J. C., Eming, S. A. (2015) Myeloid Cell-Restricted Insulin/IGF-1 Receptor Deficiency Protects against Skin Inflammation. J Immunol 195, 5296-308.

348. Mauer, J., Chaurasia, B., Plum, L., Quast, T., Hampel, B., Bluher, M., Kolanus, W., Kahn, C. R., Bruning, J. C. (2010) Myeloid cell-restricted insulin receptor deficiency protects against obesity-induced inflammation and systemic insulin resistance. PLoS Genet 6, e1000938. 
349. Fan, W., Morinaga, H., Kim, J. J., Bae, E., Spann, N. J., Heinz, S., Glass, C. K., Olefsky, J. M. (2010) FoxO1 regulates TIr4 inflammatory pathway signalling in macrophages. EMBO Journal 29, 4223-4236.

350. Su, D., Coudriet, G. M., Hyun Kim, D., Lu, Y., Perdomo, G., Qu, S., Slusher, S., Tse, H. M., Piganelli, J., Giannoukakis, N., Zhang, J., Dong, H. H. (2009) FoxO1 links insulin resistance to proinflammatory cytokine IL-1beta production in macrophages. Diabetes 58, 2624-33.

351. Chung, S., Lee, T. J., Reader, B. F., Kim, J. Y., Lee, Y. G., Park, G. Y., Karpurapu, M., Ballinger, M. N., Qian, F., Rusu, L., Chung, H. Y., Unterman, T. G., Croce, C. M., Christman, J. W. (2016) FoxO1 regulates allergic asthmatic inflammation through regulating polarization of the macrophage inflammatory phenotype. Oncotarget 7, 17532-17546.

352. Chung, S., Ranjan, R., Lee, Y. G., Park, G. Y., Karpurapu, M., Deng, J., Xiao, L., Kim, J. Y., Unterman, T. G., Christman, J. W. (2015) Distinct role of FoxO1 in M-CSF- and GM-CSF-differentiated macrophages contributes LPSmediated IL-10: implication in hyperglycemia. J Leukoc Biol 97, 327-39.

353. O'Neill, L. A. and Hardie, D. G. (2013) Metabolism of inflammation limited by AMPK and pseudo-starvation. Nature 493, 346-55.

354. Mounier, R., Theret, M., Arnold, L., Cuvellier, S., Bultot, L., Goransson, O., Sanz, N., Ferry, A., Sakamoto, K., Foretz, M., Viollet, B., Chazaud, B. (2013) AMPKalpha1 regulates macrophage skewing at the time of resolution of inflammation during skeletal muscle regeneration. Cell Metab 18, 251-64.

355. Sag, D., Carling, D., Stout, R. D., Suttles, J. (2008) Adenosine 5'monophosphate-activated protein kinase promotes macrophage polarization to an anti-inflammatory functional phenotype. J Immunol 181, 8633-41.

356. Yeung, F., Hoberg, J. E., Ramsey, C. S., Keller, M. D., Jones, D. R., Frye, R. A., Mayo, M. W. (2004) Modulation of NF-kappaB-dependent transcription and cell survival by the SIRT1 deacetylase. EMBO J 23, 2369-80.

357. Kelly, T. J., Lerin, C., Haas, W., Gygi, S. P., Puigserver, P. (2009) GCN5mediated transcriptional control of the metabolic coactivator PGC-1beta through lysine acetylation. J Biol Chem 284, 19945-52.

358. Poulose, N. and Raju, R. (2015) Sirtuin regulation in aging and injury. Biochim Biophys Acta 1852, 2442-55.

359. Gomes, A. P., Price, N. L., Ling, A. J., Moslehi, J. J., Montgomery, M. K., Rajman, L., White, J. P., Teodoro, J. S., Wrann, C. D., Hubbard, B. P., Mercken, E. M., Palmeira, C. M., de Cabo, R., Rolo, A. P., Turner, N., Bell, E. L., Sinclair, D. A. (2013) Declining NAD(+) induces a pseudohypoxic state disrupting nuclear-mitochondrial communication during aging. Cell 155, 162438.

360. Rahat, M. A., Bitterman, H., Lahat, N. (2011) Molecular mechanisms regulating macrophage response to hypoxia. Front Immunol 2, 45.

361. Cheng, S. C., Quintin, J., Cramer, R. A., Shepardson, K. M., Saeed, S., Kumar, V., Giamarellos-Bourboulis, E. J., Martens, J. H., Rao, N. A., Aghajanirefah, A., Manjeri, G. R., Li, Y., Ifrim, D. C., Arts, R. J., van der Meer, B. M., Deen, P. M., Logie, C., O'Neill, L. A., Willems, P., van de Veerdonk, F. L., van der Meer, J. W., Ng, A., Joosten, L. A., Wijmenga, C., Stunnenberg, H. G., Xavier, R. J., Netea, M. G. (2014) mTOR- and HIF-1alpha-mediated aerobic glycolysis as metabolic basis for trained immunity. Science 345, 1250684 .

362. Saeed, S., Quintin, J., Kerstens, H. H., Rao, N. A., Aghajanirefah, A., Matarese, F., Cheng, S. C., Ratter, J., Berentsen, K., van der Ent, M. A., 
Sharifi, N., Janssen-Megens, E. M., Ter Huurne, M., Mandoli, A., van Schaik, T., Ng, A., Burden, F., Downes, K., Frontini, M., Kumar, V., GiamarellosBourboulis, E. J., Ouwehand, W. H., van der Meer, J. W., Joosten, L. A., Wijmenga, C., Martens, J. H., Xavier, R. J., Logie, C., Netea, M. G., Stunnenberg, H. G. (2014) Epigenetic programming of monocyte-tomacrophage differentiation and trained innate immunity. Science 345, 1251086.

363. Kaikkonen, M. U., Spann, N. J., Heinz, S., Romanoski, C. E., Allison, K. A., Stender, J. D., Chun, H. B., Tough, D. F., Prinjha, R. K., Benner, C., Glass, C. K. (2013) Remodeling of the enhancer landscape during macrophage activation is coupled to enhancer transcription. Mol Cell 51, 310-25.

364. Stender, J. D. and Glass, C. K. (2013) Epigenomic control of the innate immune response. Curr Opin Pharmacol 13, 582-7.

365. Neele, A. E., Van den Bossche, J., Hoeksema, M. A., de Winther, M. P. (2015) Epigenetic pathways in macrophages emerge as novel targets in atherosclerosis. Eur J Pharmacol 763, 79-89.

366. De Santa, F., Narang, V., Yap, Z. H., Tusi, B. K., Burgold, T., Austenaa, L., Bucci, G., Caganova, M., Notarbartolo, S., Casola, S., Testa, G., Sung, W. K., Wei, C. L., Natoli, G. (2009) Jmjd3 contributes to the control of gene expression in LPS-activated macrophages. EMBO J 28, 3341-52.

367. De Santa, F., Totaro, M. G., Prosperini, E., Notarbartolo, S., Testa, G., Natoli, G. (2007) The histone H3 lysine-27 demethylase Jmjd3 links inflammation to inhibition of polycomb-mediated gene silencing. Cell 130, 1083-94.

368. Kruidenier, L., Chung, C. W., Cheng, Z., Liddle, J., Che, K., Joberty, G., Bantscheff, M., Bountra, C., Bridges, A., Diallo, H., Eberhard, D., Hutchinson, S., Jones, E., Katso, R., Leveridge, M., Mander, P. K., Mosley, J., RamirezMolina, C., Rowland, P., Schofield, C. J., Sheppard, R. J., Smith, J. E., Swales, C., Tanner, R., Thomas, P., Tumber, A., Drewes, G., Oppermann, U., Patel, D. J., Lee, K., Wilson, D. M. (2012) A selective jumonji H3K27 demethylase inhibitor modulates the proinflammatory macrophage response. Nature 488, 404-8.

369. Ishii, M., Wen, H., Corsa, C. A., Liu, T., Coelho, A. L., Allen, R. M., Carson, W. F. t., Cavassani, K. A., Li, X., Lukacs, N. W., Hogaboam, C. M., Dou, Y., Kunkel, S. L. (2009) Epigenetic regulation of the alternatively activated macrophage phenotype. Blood 114, 3244-54.

370. Satoh, T., Takeuchi, O., Vandenbon, A., Yasuda, K., Tanaka, Y., Kumagai, Y., Miyake, T., Matsushita, K., Okazaki, T., Saitoh, T., Honma, K., Matsuyama, T., Yui, K., Tsujimura, T., Standley, D. M., Nakanishi, K., Nakai, K., Akira, S. (2010) The Jmjd3-Irf4 axis regulates M2 macrophage polarization and host responses against helminth infection. Nat Immunol 11, 936-44.

371. Mullican, S. E., Gaddis, C. A., Alenghat, T., Nair, M. G., Giacomin, P. R., Everett, L. J., Feng, D., Steger, D. J., Schug, J., Artis, D., Lazar, M. A. (2011) Histone deacetylase 3 is an epigenomic brake in macrophage alternative activation. Genes Dev 25, 2480-8.

372. Hoeksema, M. A., Gijbels, M. J., Van den Bossche, J., van der Velden, S., Sijm, A., Neele, A. E., Seijkens, T., Stoger, J. L., Meiler, S., Boshuizen, M. C., Dallinga-Thie, G. M., Levels, J. H., Boon, L., Mullican, S. E., Spann, N. J., Cleutjens, J. P., Glass, C. K., Lazar, M. A., de Vries, C. J., Biessen, E. A., Daemen, M. J., Lutgens, E., de Winther, M. P. (2014) Targeting macrophage Histone deacetylase 3 stabilizes atherosclerotic lesions. EMBO Mol Med 6, 1124-32. 
373. Plank, J. L. and Dean, A. (2014) Enhancer function: mechanistic and genomewide insights come together. Mol Cell 55, 5-14.

374. Wu, H. and Zhang, Y. (2014) Reversing DNA methylation: mechanisms, genomics, and biological functions. Cell 156, 45-68.

375. Jung, M. and Pfeifer, G. P. (2015) Aging and DNA methylation. BMC Biol 13, 7.

376. Horvath, S. (2013) DNA methylation age of human tissues and cell types. Genome Biol 14, R115.

377. Hannum, G., Guinney, J., Zhao, L., Zhang, L., Hughes, G., Sadda, S., Klotzle, B., Bibikova, M., Fan, J. B., Gao, Y., Deconde, R., Chen, M., Rajapakse, I., Friend, S., Ideker, T., Zhang, K. (2013) Genome-wide methylation profiles reveal quantitative views of human aging rates. Mol Cell 49, 359-67.

378. Kaelin, W. G., Jr. and McKnight, S. L. (2013) Influence of metabolism on epigenetics and disease. Cell 153, 56-69.

379. Sutendra, G., Kinnaird, A., Dromparis, P., Paulin, R., Stenson, T. H., Haromy, A., Hashimoto, K., Zhang, N., Flaim, E., Michelakis, E. D. (2014) A nuclear pyruvate dehydrogenase complex is important for the generation of acetylCoA and histone acetylation. Cell 158, 84-97.

380. Baardman, J., Licht, I., de Winther, M. P., Van den Bossche, J. (2015) Metabolic-epigenetic crosstalk in macrophage activation. Epigenomics 7, 1155-64.

381. Mentch, S. J. and Locasale, J. W. (2016) One-carbon metabolism and epigenetics: understanding the specificity. Ann N Y Acad Sci 1363, 91-8.

382. Vinolo, M. A., Rodrigues, H. G., Nachbar, R. T., Curi, R. (2011) Regulation of inflammation by short chain fatty acids. Nutrients 3, 858-76.

383. Davie, J. R. (2003) Inhibition of histone deacetylase activity by butyrate. J Nutr 133, 2485S-2493S.

384. Li, P., Spann, N. J., Kaikkonen, M. U., Lu, M., Oh da, Y., Fox, J. N., Bandyopadhyay, G., Talukdar, S., Xu, J., Lagakos, W. S., Patsouris, D., Armando, A., Quehenberger, O., Dennis, E. A., Watkins, S. M., Auwerx, J., Glass, C. K., Olefsky, J. M. (2013) NCoR repression of LXRs restricts macrophage biosynthesis of insulin-sensitizing omega 3 fatty acids. Cell 155, 200-14.

385. Xue, B., Yang, Z., Wang, X., Shi, H. (2012) Omega-3 polyunsaturated fatty acids antagonize macrophage inflammation via activation of AMPK/SIRT1 pathway. PLoS One 7, e45990.

386. Spaulding, C. C., Walford, R. L., Effros, R. B. (1997) Calorie restriction inhibits the age-related dysregulation of the cytokines TNF-alpha and IL-6 in C3B10RF1 mice. Mech Ageing Dev 93, 87-94.

387. Jolly, C. A. (2004) Dietary restriction and immune function. J Nutr 134, 18536.

388. Starr, M. E., Steele, A. M., Cohen, D. A., Saito, H. (2016) Short-Term Dietary Restriction Rescues Mice From Lethal Abdominal Sepsis and Endotoxemia and Reduces the Inflammatory/Coagulant Potential of Adipose Tissue. Crit Care Med 44, e509-519.

389. Wanders, D., Ghosh, S., Stone, K. P., Van, N. T., Gettys, T. W. (2014) Transcriptional impact of dietary methionine restriction on systemic inflammation: relevance to biomarkers of metabolic disease during aging. Biofactors 40, 13-26.

390. Ohashi, K., Parker, J. L., Ouchi, N., Higuchi, A., Vita, J. A., Gokce, N., Pedersen, A. A., Kalthoff, C., Tullin, S., Sams, A., Summer, R., Walsh, K. 
(2010) Adiponectin promotes macrophage polarization toward an antiinflammatory phenotype. J Biol Chem 285, 6153-60.

391. Ingram, D. K., Anson, R. M., de Cabo, R., Mamczarz, J., Zhu, M., Mattison, J., Lane, M. A., Roth, G. S. (2004) Development of calorie restriction mimetics as a prolongevity strategy. Ann N Y Acad Sci 1019, 412-23.

392. Pacheco, S. A., Powers, K. M., Engelmann, F., Messaoudi, I., Purdy, G. E. (2013) Autophagic Killing Effects against by Alveolar Macrophages from Young and Aged Rhesus Macaques. PLoS One 8, e66985.

393. Kim, M. Y., Lim, J. H., Youn, H. H., Hong, Y. A., Yang, K. S., Park, H. S., Chung, S., Ko, S. H., Shin, S. J., Choi, B. S., Kim, H. W., Kim, Y. S., Lee, J. H., Chang, Y. S., Park, C. W. (2013) Resveratrol prevents renal lipotoxicity and inhibits mesangial cell glucotoxicity in a manner dependent on the AMPKSIRT1-PGC1alpha axis in db/db mice. Diabetologia 56, 204-17.

394. Carreras, A., Zhang, S. X., Almendros, I., Wang, Y., Peris, E., Qiao, Z., Gozal, D. (2015) Resveratrol attenuates intermittent hypoxia-induced macrophage migration to visceral white adipose tissue and insulin resistance in male mice. Endocrinology 156, 437-43.

395. van der Goot, A. T. and Nollen, E. A. (2013) Tryptophan metabolism: entering the field of aging and age-related pathologies. Trends Mol Med 19, 336-44.

396. Wang, X. F., Wang, H. S., Wang, H., Zhang, F., Wang, K. F., Guo, Q., Zhang, G., Cai, S. H., Du, J. (2014) The role of indoleamine 2,3-dioxygenase (IDO) in immune tolerance: focus on macrophage polarization of THP-1 cells. Cell Immunol 289, 42-8.

397. Zhu, L., Zhao, Q., Yang, T., Ding, W., Zhao, Y. (2015) Cellular metabolism and macrophage functional polarization. Int Rev Immunol 34, 82-100.

398. Van den Bossche, J., Lamers, W. H., Koehler, E. S., Geuns, J. M., Alhonen, L., Uimari, A., Pirnes-Karhu, S., Van Overmeire, E., Morias, Y., Brys, L., Vereecke, L., De Baetselier, P., Van Ginderachter, J. A. (2012) Pivotal Advance: Arginase-1-independent polyamine production stimulates the expression of IL-4-induced alternatively activated macrophage markers while inhibiting LPS-induced expression of inflammatory genes. J Leukoc Biol 91, 685-99.

399. Soda, K., Dobashi, Y., Kano, Y., Tsujinaka, S., Konishi, F. (2009) Polyaminerich food decreases age-associated pathology and mortality in aged mice. Exp Gerontol 44, 727-32.

400. Woods, J. A., Wilund, K. R., Martin, S. A., Kistler, B. M. (2012) Exercise, inflammation and aging. Aging Dis 3, 130-40.

401. Kawanishi, N., Yano, H., Yokogawa, Y., Suzuki, K. (2010) Exercise training inhibits inflammation in adipose tissue via both suppression of macrophage infiltration and acceleration of phenotypic switching from M1 to M2 macrophages in high-fat-diet-induced obese mice. Exerc Immunol Rev 16, 105-18.

402. Guerrieri, D. and van Praag, H. (2015) Exercise-mimetic AICAR transiently benefits brain function. Oncotarget 6, 18293-313.

403. Narkar, V. A., Downes, M., Yu, R. T., Embler, E., Wang, Y. X., Banayo, E., Mihaylova, M. M., Nelson, M. C., Zou, Y., Juguilon, H., Kang, H., Shaw, R. J., Evans, R. M. (2008) AMPK and PPARdelta agonists are exercise mimetics. Cell 134, 405-15.

404. Ding, L., Liang, G., Yao, Z., Zhang, J., Liu, R., Chen, H., Zhou, Y., Wu, H., Yang, B., He, Q. (2015) Metformin prevents cancer metastasis by inhibiting 
M2-like polarization of tumor associated macrophages. Oncotarget 6, 3644155.

405. Namgaladze, D., Kemmerer, M., von Knethen, A., Brune, B. (2013) AICAR inhibits PPARgamma during monocyte differentiation to attenuate inflammatory responses to atherogenic lipids. Cardiovasc Res 98, 479-87.

406. Sharpless, N. E. and Sherr, C. J. (2015) Forging a signature of in vivo senescence. Nat Rev Cancer 15, 397-408.

407. Man, A. L., Bertelli, E., Rentini, S., Regoli, M., Briars, G., Marini, M., Watson, A. J., Nicoletti, C. (2015) Age-associated modifications of intestinal permeability and innate immunity in human small intestine. Clin Sci (Lond) 129, 515-27.

408. Xue, J., Schmidt, S. V., Sander, J., Draffehn, A., Krebs, W., Quester, I., De Nardo, D., Gohel, T. D., Emde, M., Schmidleithner, L., Ganesan, H., NinoCastro, A., Mallmann, M. R., Labzin, L., Theis, H., Kraut, M., Beyer, M., Latz, E., Freeman, T. C., Ulas, T., Schultze, J. L. (2014) Transcriptome-based network analysis reveals a spectrum model of human macrophage activation. Immunity 40, 274-88.

409. Hoenicke, L. and Zender, L. (2012) Immune surveillance of senescent cells-biological significance in cancer- and non-cancer pathologies. Carcinogenesis 33, 1123-6.

410. Baker, D. J., Childs, B. G., Durik, M., Wijers, M. E., Sieben, C. J., Zhong, J., Saltness, R. A., Jeganathan, K. B., Verzosa, G. C., Pezeshki, A., Khazaie, K., Miller, J. D., van Deursen, J. M. (2016) Naturally occurring p16(Ink4a)-positive cells shorten healthy lifespan. Nature 530, 184-9.

411. Kong, K. F., Delroux, K., Wang, X., Qian, F., Arjona, A., Malawista, S. E., Fikrig, E., Montgomery, R. R. (2008) Dysregulation of TLR3 impairs the innate immune response to West Nile virus in the elderly. J Virol 82, 7613-23.

412. Przybyla, B., Gurley, C., Harvey, J. F., Bearden, E., Kortebein, P., Evans, W. J., Sullivan, D. H., Peterson, C. A., Dennis, R. A. (2006) Aging alters macrophage properties in human skeletal muscle both at rest and in response to acute resistance exercise. Exp Gerontol 41, 320-7.

413. Zhao, H., Roychoudhury, J., Doggett, T. A., Apte, R. S., Ferguson, T. A. (2013) Age-dependent changes in FasL (CD95L) modulate macrophage function in a model of age-related macular degeneration. Invest Ophthalmol Vis Sci 54, 5321-31.

414. Poliani, P. L., Wang, Y., Fontana, E., Robinette, M. L., Yamanishi, Y., Gilfillan, S., Colonna, M. (2015) TREM2 sustains microglial expansion during aging and response to demyelination. J Clin Invest 125, 2161-70.

415. Sierra, A., Gottfried-Blackmore, A. C., McEwen, B. S., Bulloch, K. (2007) Microglia derived from aging mice exhibit an altered inflammatory profile. Glia $55,412-24$.

416. Fenn, A. M., Henry, C. J., Huang, Y., Dugan, A., Godbout, J. P. (2012) Lipopolysaccharide-induced interleukin (IL)-4 receptor-alpha expression and corresponding sensitivity to the M2 promoting effects of IL-4 are impaired in microglia of aged mice. Brain Behav Immun 26, 766-77.

417. Wynne, A. M., Henry, C. J., Huang, Y., Cleland, A., Godbout, J. P. (2010) Protracted downregulation of CX3CR1 on microglia of aged mice after lipopolysaccharide challenge. Brain Behav Immun 24, 1190-201.

418. Kelly, J., Ali Khan, A., Yin, J., Ferguson, T. A., Apte, R. S. (2007) Senescence regulates macrophage activation and angiogenic fate at sites of tissue injury in mice. J Clin Invest 117, 3421-6. 
419. Kohut, M. L., Senchina, D. S., Madden, K. S., Martin, A. E., Felten, D. L., Moynihan, J. A. (2004) Age effects on macrophage function vary by tissue site, nature of stimulant, and exercise behavior. Exp Gerontol 39, 1347-60.

420. Rottinghaus, E. K., Vesosky, B., Turner, J. (2010) TLR-2 independent recognition of Mycobacterium tuberculosis by CD11C+ pulmonary cells from old mice. Mech Ageing Dev 131, 405-14.

421. Canan, C. H., Gokhale, N. S., Carruthers, B., Lafuse, W. P., Schlesinger, L. S., Torrelles, J. B., Turner, J. (2014) Characterization of lung inflammation and its impact on macrophage function in aging. $J$ Leukoc Biol 96, 473-80.

422. Hinojosa, C. A., Akula Suresh Babu, R., Rahman, M. M., Fernandes, G., Boyd, A. R., Orihuela, C. J. (2014) Elevated A20 contributes to agedependent macrophage dysfunction in the lungs. Exp Gerontol 54, 58-66.

423. Ren, X., Du, H., Li, Y., Yao, X., Huang, J., Li, Z., Wang, W., Li, J., Han, S., Wang, C., Huang, K. (2014) Age-related activation of MKK/p38/NF-kappaB signaling pathway in lung: from mouse to human. Exp Gerontol 57, 29-40.

424. Takahashi, R., Totsuka, S., Ishigami, A., Kobayashi, Y., Nagata, K. (2016) Attenuated phagocytosis of secondary necrotic neutrophils by macrophages in aged and SMP30 knockout mice. Geriatr Gerontol Int 16, 135-142.

425. Wu, D., Ren, Z., Pae, M., Han, S. N., Meydani, S. N. (2013) Diet-induced obesity has a differential effect on adipose tissue and macrophage inflammatory responses of young and old mice. Biofactors 39, 326-33.

426. Yoon, P., Keylock, K. T., Hartman, M. E., Freund, G. G., Woods, J. A. (2004) Macrophage hypo-responsiveness to interferon-gamma in aged mice is associated with impaired signaling through Jak-STAT. Mech Ageing Dev 125, 137-43.

427. Murciano, C., Yanez, A., O'Connor, J. E., Gozalbo, D., Gil, M. L. (2008) Influence of aging on murine neutrophil and macrophage function against Candida albicans. FEMS Immunol Med Microbiol 53, 214-21.

428. Lim, J. S., Nguyen, K. C., Nguyen, C. T., Jang, I. S., Han, J. M., Fabian, C., Lee, S. E., Rhee, J. H., Cho, K. A. (2015) Flagellin-dependent TLR5/caveolin1 as a promising immune activator in immunosenescence. Aging Cell 14, 90715.

429. Liang, S., Domon, H., Hosur, K. B., Wang, M., Hajishengallis, G. (2009) Agerelated alterations in innate immune receptor expression and ability of macrophages to respond to pathogen challenge in vitro. Mech Ageing Dev 130, 538-46.

430. Swift, M. E., Burns, A. L., Gray, K. L., DiPietro, L. A. (2001) Age-related alterations in the inflammatory response to dermal injury. $J$ Invest Dermatol 117, 1027-35.

431. Chelvarajan, R. L., Collins, S. M., Van Willigen, J. M., Bondada, S. (2005) The unresponsiveness of aged mice to polysaccharide antigens is a result of a defect in macrophage function. J Leukoc Biol 77, 503-12.

432. Fallah, M. P., Chelvarajan, R. L., Garvy, B. A., Bondada, S. (2011) Role of phosphoinositide 3-kinase-Akt signaling pathway in the age-related cytokine dysregulation in splenic macrophages stimulated via TLR-2 or TLR-4 receptors. Mech Ageing Dev 132, 274-86.

433. Wu, D., Ren, Z., Pae, M., Guo, W., Cui, X., Merrill, A. H., Meydani, S. N. (2007) Aging up-regulates expression of inflammatory mediators in mouse adipose tissue. J Immunol 179, 4829-39.

434. Ley, R. E., Peterson, D. A., Gordon, J. I. (2006) Ecological and evolutionary forces shaping microbial diversity in the human intestine. Cell 124, 837-848. 
435. Borchers, A. T., Selmi, C., Meyers, F. J., Keen, C. L., Gershwin, M. E. (2009) Probiotics and immunity. Journal of Gastroenterology 44, 26-46.

436. Heintz, C. and Mair, W. (2014) You are what you host: microbiome modulation of the aging process. Cell 156, 408-411.

437. Chieppa, M., Rescigno, M., Huang, A. Y., Germain, R. N. (2006) Dynamic imaging of dendritic cell extension into the small bowel lumen in response to epithelial cell TLR engagement. The Journal of Experimental Medicine 203, 2841-2852.

438. Farache, J., Koren, I., Milo, I., Gurevich, I., Kim, K.-W., Zigmond, E., Furtado, G. C., Lira, S. A., Shakhar, G. (2013) Luminal Bacteria Recruit CD103< sup $>+</$ sup $>$ Dendritic Cells into the Intestinal Epithelium to Sample Bacterial Antigens for Presentation. Immunity 38, 581-595.

439. Rescigno, M., Urbano, M., Valzasina, B., Francolini, M., Rotta, G., Bonasio, R., Granucci, F., Kraehenbuhl, J.-P., Ricciardi-Castagnoli, P. (2001) Dendritic cells express tight junction proteins and penetrate gut epithelial monolayers to sample bacteria. Nature Immunology 2, 361-367.

440. Murray, P. J., Allen, J. E., Biswas, S. K., Fisher, E. A., Gilroy, D. W., Goerdt, S., Gordon, S., Hamilton, J. A., Ivashkiv, L. B., Lawrence, T. (2014) Macrophage activation and polarization: nomenclature and experimental guidelines. Immunity 41, 14-20.

441. Vesa, T., Pochart, P., Marteau, P. (2000) Pharmacokinetics of Lactobacillus plantarum NCIMB 8826, Lactobacillus fermentum KLD, and Lactococcus lactis MG 1363 in the human gastrointestinal tract. Alimentary Pharmacology and Therapeutics 14, 823-828.

442. Bäuerl, C., Pérez-Martínez, G., Yan, F., Polk, D. B., Monedero, V. (2010) Functional analysis of the p40 and p75 proteins from Lactobacillus casei BL23. Journal of Molecular Microbiology and Biotechnology 19, 231-241.

443. Mazé, A., Boël, G., Zúñiga, M., Bourand, A., Loux, V., Yebra, M. J., Monedero, V., Correia, K., Jacques, N., Beaufils, S. (2010) Complete genome sequence of the probiotic Lactobacillus casei strain BL23. Journal of Bacteriology 192, 2647-2648.

444. Foligne, B., Nutten, S., Grangette, C., Dennin, V., Goudercourt, D., Poiret, S., Dewulf, J., Brassart, D., Mercenier, A., Pot, B. (2007) Correlation between in vitro and in vivo immunomodulatory properties of lactic acid bacteria. World Journal of Gastroenterology 13, 236-243.

445. Wegmann, U., O'Connell-Motherway, M., Zomer, A., Buist, G., Shearman, C., Canchaya, C., Ventura, M., Goesmann, A., Gasson, M. J., Kuipers, O. P. (2007) Complete genome sequence of the prototype lactic acid bacterium Lactococcus lactis subsp. cremoris MG1363. Journal of Bacteriology 189, 3256-3270.

446. Hougee, S., Vriesema, A., Wijering, S., Knippels, L., Folkerts, G., Nijkamp, F., Knol, J., Garssen, J. (2009) Oral treatment with probiotics reduces allergic symptoms in ovalbumin-sensitized mice: a bacterial strain comparative study. International Archives of Allergy and Immunology 151, 107-117.

447. Ewaschuk, J. B., Diaz, H., Meddings, L., Diederichs, B., Dmytrash, A., Backer, J., Looijer-van Langen, M., Madsen, K. L. (2008) Secreted bioactive factors from Bifidobacterium infantis enhance epithelial cell barrier function. American Journal of Physiology-Gastrointestinal and Liver Physiology 295, G1025G1034.

448. Swidsinski, A., Dörffel, Y., Loening-Baucke, V., Theissig, F., Rückert, J. C., Ismail, M., Rau, W. A., Gaschler, D., Weizenegger, M., Kühn, S. (2011) Acute 
appendicitis is characterized by local invasion with Fusobacterium nucleatum/necrophorum. Gut 60, 34-40.

449. van Passel, M. W., Kant, R., Zoetendal, E. G., Plugge, C. M., Derrien, M., Malfatti, S. A., Chain, P. S., Woyke, T., Palva, A., de Vos, W. M. (2011) The genome of Akkermansia muciniphila, a dedicated intestinal mucin degrader, and its use in exploring intestinal metagenomes. PLoS ONE 6, e16876.

450. Belzer, C. and de Vos, W. M. (2012) Microbes inside-from diversity to function: the case of Akkermansia. The ISME Journal 6, 1449-1458.

451. Daniel, C., Repa, A., Wild, C., Pollak, A., Pot, B., Breiteneder, H., Wiedermann, U., Mercenier, A. (2006) Modulation of allergic immune responses by mucosal application of recombinant lactic acid bacteria producing the major birch pollen allergen Bet v 1. Allergy 61, 812-819.

452. Derrien, M., Van Baarlen, P., Hooiveld, G., Norin, E., Müller, M., de Vos, W. M. (2011) Modulation of mucosal immune response, tolerance, and proliferation in mice colonized by the mucin-degrader Akkermansia muciniphila. Frontiers in Microbiology 2, 1-14.

453. Ivanovic, N., Minic, R., Dimitrijevic, L., Skodric, S. R., Zivkovic, I., Djordjevic, B. (2015) Lactobacillus rhamnosus LA68 and Lactobacillus plantarum WCFS1 differently influence metabolic and immunological parameters in high fat dietinduced hypercholesterolemia and hepatic steatosis. Food \& Function 6, 558565.

454. Kleerebezem, M., Boekhorst, J., van Kranenburg, R., Molenaar, D., Kuipers, O. P., Leer, R., Tarchini, R., Peters, S. A., Sandbrink, H. M., Fiers, M. W. (2003) Complete genome sequence of Lactobacillus plantarum WCFS1. Proceedings of the National Academy of Sciences of the United States of America 100, 1990-1995.

455. Marco, M. L., Peters, T. H., Bongers, R. S., Molenaar, D., Van Hemert, S., Sonnenburg, J. L., Gordon, J. I., Kleerebezem, M. (2009) Lifestyle of Lactobacillus plantarum in the mouse caecum. Environmental Microbiology 11, 2747-2757.

456. Meijerink, M., Wells, J. M., Taverne, N., Zeeuw Brouwer, M. L., Hilhorst, B., Venema, K., Bilsen, J. (2012) Immunomodulatory effects of potential probiotics in a mouse peanut sensitization model. FEMS Immunology \& Medical Microbiology 65, 488-496.

457. Snel, J., Vissers, Y., Smit, B., Jongen, J., Van der Meulen, E., Zwijsen, R., Ruinemans-Koerts, J., Jansen, A., Kleerebezem, M., Savelkoul, H. (2011) Strain-specific immunomodulatory effects of Lactobacillus plantarum strains on birch-pollen-allergic subjects out of season. Clinical \& Experimental Allergy 41, 232-242.

458. Repa, A., Grangette, C., Daniel, C., Hochreiter, R., Hoffmann-Sommergruber, K., Thalhamer, J., Kraft, D., Breiteneder, H., Mercenier, A., Wiedermann, U. (2003) Mucosal co-application of lactic acid bacteria and allergen induces counter-regulatory immune responses in a murine model of birch pollen allergy. Vaccine 22, 87-95.

459. Foligne, B., Dessein, R., Marceau, M., Poiret, S., Chamaillard, M., Pot, B., Simonet, M., Daniel, C. (2007) Prevention and Treatment of Colitis With Lactococcus lactis Secreting the Immunomodulatory Yersinia LcrV Protein. Gastroenterology 133, 862-874.

460. Smelt, M. J., de Haan, B. J., Bron, P. A., van Swam, I., Meijerink, M., Wells, J. M., Faas, M. M., de Vos, P. (2012) L. plantarum, L. salivarius, and L. lactis 
attenuate Th2 responses and increase Treg frequencies in healthy mice in a strain dependent manner. PLoS ONE 7, e47244.

461. Matsumoto, T., Ishikawa, H., Tateda, K., Yaeshima, T., Ishibashi, N., Yamaguchi, K. (2008) Oral administration of Bifidobacterium longum prevents gut-derived Pseudomonas aeruginosa sepsis in mice. Journal of Applied Microbiology 104, 672-680.

462. Everard, A., Belzer, C., Geurts, L., Ouwerkerk, J. P., Druart, C., Bindels, L. B., Guiot, Y., Derrien, M., Muccioli, G. G., Delzenne, N. M. (2013) Cross-talk between Akkermansia muciniphila and intestinal epithelium controls dietinduced obesity. Proceedings of the National Academy of Sciences of the United States of America 110, 9066-9071.

463. Shin, N.-R., Lee, J.-C., Lee, H.-Y., Kim, M.-S., Whon, T. W., Lee, M.-S., Bae, J.-W. (2014) An increase in the Akkermansia spp. population induced by metformin treatment improves glucose homeostasis in diet-induced obese mice. Gut 63, 727-735.

464. Kang, C.-s., Ban, M., Choi, E.-J., Moon, H.-G., Jeon, J.-S., Kim, D.-K., Park, S.-K., Jeon, S. G., Roh, T.-Y., Myung, S.-J. (2013) Extracellular vesicles derived from gut microbiota, especially Akkermansia muciniphila, protect the progression of dextran sulfate sodium-induced colitis. PLoS ONE 8, e76520.

465. Muñoz, J. A. M., Chenoll, E., Casinos, B., Bataller, E., Ramón, D., Genovés, S., Montava, R., Ribes, J. M., Buesa, J., Fàbrega, J. (2011) Novel probiotic Bifidobacterium longum subsp. infantis CECT 7210 strain active against rotavirus infections. Applied and Environmental Microbiology 77, 8775-8783.

466. Osman, N., Adawi, D., Molin, G., Ahrne, S., Berggren, A., Jeppsson, B. (2006) Bifidobacterium infantis strains with and without a combination of oligofructose and inulin (OFI) attenuate inflammation in DSS-induced colitis in rats. BMC Gastroenterology 6, 31.

467. Derrien, M., Vaughan, E. E., Plugge, C. M., de Vos, W. M. (2004) Akkermansia muciniphila gen. nov., sp. nov., a human intestinal mucindegrading bacterium. International Journal of Systematic and Evolutionary Microbiology 54, 1469-1476.

468. Sklar, M. D., Tereba, A., Chen, B. D. M., Walker, W. S. (1985) Transformation of mouse bone marrow cells by transfection with a human oncogene related to c-myc is associated with the endogenous production of macrophage colony stimulating factor 1. Journal of Cellular Physiology 125, 403-412.

469. Hayes, M. P., Wang, J., Norcross, M. A. (1995) Regulation of interleukin-12 expression in human monocytes: selective priming by interferon-gamma of lipopolysaccharide-inducible p35 and p40 genes. Blood 86, 646-650.

470. Yi, A.-K., Yoon, J.-G., Yeo, S.-J., Hong, S.-C., English, B. K., Krieg, A. M. (2002) Role of mitogen-activated protein kinases in CpG DNA-mediated IL-10 and IL-12 production: central role of extracellular signal-regulated kinase in the negative feedback loop of the CpG DNA-mediated Th1 response. The Journal of Immunology 168, 4711-4720.

471. Meijerink, M., Van Hemert, S., Taverne, N., Wels, M., De Vos, P., Bron, P. A., Savelkoul, H. F., van Bilsen, J., Kleerebezem, M., Wells, J. M. (2010) Identification of genetic loci in Lactobacillus plantarum that modulate the immune response of dendritic cells using comparative genome hybridization. PLoS ONE 5, e10632.

472. van Hemert, S., Meijerink, M., Molenaar, D., Bron, P. A., de Vos, P., Kleerebezem, M., Wells, J. M., Marco, M. L. (2010) Identification of 
Lactobacillus plantarum genes modulating the cytokine response of human peripheral blood mononuclear cells. BMC Microbiology 10, 293.

473. Abdi, K., Singh, N. J., Spooner, E., Kessler, B. M., Radaev, S., Lantz, L., Xiao, T. S., Matzinger, P., Sun, P. D., Ploegh, H. L. (2014) Free IL-12p40 Monomer Is a Polyfunctional Adaptor for Generating Novel IL-12-like Heterodimers Extracellularly. The Journal of Immunology 192, 6028-6036.

474. Gee, K., Guzzo, C., Mat, C., Nor, F., Ma, W., Kumar, A. (2009) The IL-12 family of cytokines in infection, inflammation and autoimmune disorders. Inflammation \& Allergy-Drug Targets 8, 40-52.

475. Kollmann, T. R., Levy, O., Montgomery, R. R., Goriely, S. (2012) Innate immune function by Toll-like receptors: distinct responses in newborns and the elderly. Immunity $37,771-783$.

476. Boehmer, E. D., Meehan, M. J., Cutro, B. T., Kovacs, E. J. (2005) Aging negatively skews macrophage TLR2-and TLR4-mediated pro-inflammatory responses without affecting the IL-2-stimulated pathway. Mechanisms of Ageing and Development 126, 1305-1313.

477. Chelvarajan, R. L., Liu, Y., Popa, D., Getchell, M. L., Getchell, T. V., Stromberg, A. J., Bondada, S. (2006) Molecular basis of age-associated cytokine dysregulation in LPS-stimulated macrophages. Journal of Leukocyte Biology 79, 1314-1327.

478. Jiang, M., Xiang, Y., Wang, D., Gao, J., Liu, D., Liu, Y., Liu, S., Zheng, D. (2012) Dysregulated expression of miR-146a contributes to age-related dysfunction of macrophages. Aging Cell 11, 29-40.

479. Sebastián, C., Espia, M., Serra, M., Celada, A., Lloberas, J. (2005) MacrophAging: a cellular and molecular review. Immunobiology 210, 121-126.

480. Górska, S., Schwarzer, M., Jachymek, W., Srutkova, D., Brzozowska, E., Kozakova, H., Gamian, A. (2014) Distinct Immunomodulation of Bone Marrow-Derived Dendritic Cell Responses to Lactobacillus plantarum WCFS1 by Two Different Polysaccharides Isolated from Lactobacillus rhamnosus LOCK 0900. Applied and Environmental Microbiology 80, 6506-6516.

481. Li, Z., Liu, C., Xie, Z., Song, P., Zhao, R. C., Guo, L., Liu, Z., Wu, Y. (2011) Epigenetic dysregulation in mesenchymal stem cell aging and spontaneous differentiation. PLoS ONE 6, e20526.

482. Warren, L. A. and Rossi, D. J. (2009) Stem cells and aging in the hematopoietic system. Mechanisms of Ageing and Development 130, 46-53.

483. Olivieri, F., Rippo, M. R., Prattichizzo, F., Babini, L., Graciotti, L., Recchioni, R., Procopio, A. D. (2013) Toll like receptor signaling in "inflammaging": microRNA as new players. Immunity \& Ageing 10.

484. Sun, D., Luo, M., Jeong, M., Rodriguez, B., Xia, Z., Hannah, R., Wang, H., Le, T., Faull, K. F., Chen, R. (2014) Epigenomic profiling of young and aged HSCs reveals concerted changes during aging that reinforce self-renewal. Cell Stem Cell 14, 673-688.

485. Kanatsu-Shinohara, M., Ogonuki, N., Iwano, T., Lee, J., Kazuki, Y., Inoue, K., Miki, H., Takehashi, M., Toyokuni, S., Shinkai, Y. (2005) Genetic and epigenetic properties of mouse male germline stem cells during long-term culture. Development 132, 4155-4163.

486. Man, A. L., Gicheva, N., Nicoletti, C. (2014) The impact of ageing on the intestinal epithelial barrier and immune system. Cellular Immunology 289, 112-118. 
487. Johansson, M. E., Larsson, J. M. H., Hansson, G. C. (2011) The two mucus layers of colon are organized by the MUC2 mucin, whereas the outer layer is a legislator of host-microbial interactions. Proceedings of the national academy of sciences $108,4659-4665$.

488. George, A. J. and Ritter, M. A. (1996) Thymic involution with ageing: obsolescence or good housekeeping? Immunology Today 17, 267-272.

489. Akatsu, H., Iwabuchi, N., Xiao, J.-z., Matsuyama, Z., Kurihara, R., Okuda, K., Yamamoto, T., Maruyama, M. (2012) Clinical effects of probiotic Bifidobacterium longum BB536 on immune function and intestinal microbiota in elderly patients receiving enteral tube feeding. Journal of Parenteral and Enteral Nutrition 37, 631-640.

490. Spaiser, S. J., Culpepper, T., Nieves Jr, C., Ukhanova, M., Mai, V., Percival, S. S., Christman, M. C., Langkamp-Henken, B. (2015) Lactobacillus gasseri KS-13, Bifidobacterium bifidum G9-1, and Bifidobacterium longum MM-2 Ingestion Induces a Less Inflammatory Cytokine Profile and a Potentially Beneficial Shift in Gut Microbiota in Older Adults: A Randomized, DoubleBlind, Placebo-Controlled, Crossover Study. Journal of the American College of Nutrition 34, 459-469.

491. Rampelli, S., Candela, M., Severgnini, M., Biagi, E., Turroni, S., Roselli, M., Carnevali, P., Donini, L., Brigidi, P. (2013) A probiotics-containing biscuit modulates the intestinal microbiota in the elderly. The Journal of Nutrition, Health \& Aging 17, 166-172.

492. Gill, H., Rutherfurd, K., Cross, M. (2001) Dietary probiotic supplementation enhances natural killer cell activity in the elderly: an investigation of agerelated immunological changes. Journal of Clinical Immunology 21, 264-271.

493. Gill, H. S., Rutherfurd, K. J., Cross, M. L., Gopal, P. K. (2001) Enhancement of immunity in the elderly by dietary supplementation with the probiotic Bifidobacterium lactis HN019. The American Journal of Clinical Nutrition 74, 833-839.

494. Vidal, K., Benyacoub, J., Moser, M., Sanchez-Garcia, J., Serrant, P., SeguraRoggero, I., Reuteler, G., Blum, S. (2008) Effect of Lactobacillus paracasei NCC2461 on antigen-specific T-cell mediated immune responses in aged mice. Rejuvenation Research 11, 957-964.

495. Gurkar, A. U. and Niedernhofer, L. J. (2015) Comparison of mice with accelerated aging caused by distinct mechanisms. Experimental Gerontology 68, 43-50.

496. van Beek, A. A., Hugenholtz, F., Meijer, B., Sovran, B., Perdijk, O., Vermeij, W. P., Brandt, R. M., Barnhoorn, S., Hoeijmakers, J. H., de Vos, P., Leenen, P. J., Hendriks, R. W., Savelkoul, H. (2016) Tryptophan restriction arrests B cell development and enhances microbial diversity in WT and prematurely aging Ercc1-/ $\Delta 7$ mice. Journal of Leukocyte Biology In press.

497. Van Beek, A. A., Hoogerland, J. A., Belzer, C., De Vos, P., De Vos, W., Savelkoul, H. F., Leenen, P. J. (2016) Interaction of mouse splenocytes and macrophages with bacterial strains in vivo: the effect of age in the immune response. Beneficial Microbes 7, 275-287.

498. Cho, J. S., Kook, S. H., Robinson, A. R., Niedernhofer, L. J., Lee, B. C. (2013) Cell autonomous and nonautonomous mechanisms drive hematopoietic stem/progenitor cell loss in the absence of DNA repair. Stem Cells 31, 511525.

499. Sovran, B., Lu, P., Loonen, L. M., Hugenholtz, F., Belzer, C., Stolte, E. H., Boekschoten, M. V., van Baarlen, P., Smidt, H., Kleerebezem, M. (2016) 
Identification of Commensal Species Positively Correlated with Early Stress Responses to a Compromised Mucus Barrier. Inflammatory Bowel Diseases 22, 826-840.

500. Rajilić-Stojanović, M., Heilig, H. G., Molenaar, D., Kajander, K., Surakka, A., Smidt, H., De Vos, W. M. (2009) Development and application of the human intestinal tract chip, a phylogenetic microarray: analysis of universally conserved phylotypes in the abundant microbiota of young and elderly adults. Environmental Microbiology 11, 1736-1751.

501. Lahti, L., Elo, L. L., Aittokallio, T., Kaski, S. (2011) Probabilistic analysis of probe reliability in differential gene expression studies with short oligonucleotide arrays. IEEE/ACM Transactions on Computational Biology and Bioinformatics (TCBB) 8, 217-225.

502. Braak, C. J. F. and Šmilauer, P. (2012) CANOCO reference manual and user's guide: software for ordination (version 5.0). Biometris.

503. van Loo, P. F., Dingjan, G. M., Maas, A., Hendriks, R. W. (2007) Surrogatelight-chain silencing is not critical for the limitation of pre-B cell expansion but is for the termination of constitutive signaling. Immunity $27,468-480$.

504. Subramanian, A., Tamayo, P., Mootha, V. K., Mukherjee, S., Ebert, B. L., Gillette, M. A., Paulovich, A., Pomeroy, S. L., Golub, T. R., Lander, E. S. (2005) Gene set enrichment analysis: a knowledge-based approach for interpreting genome-wide expression profiles. Proceedings of the National Academy of Sciences of the United States of America 102, 15545-15550.

505. Pretzer, G., Snel, J., Molenaar, D., Wiersma, A., Bron, P. A., Lambert, J., de Vos, W. M., van der Meer, R., Smits, M. A., Kleerebezem, M. (2005) Biodiversity-based identification and functional characterization of the mannose-specific adhesin of Lactobacillus plantarum. Journal of Bacteriology 187, 6128-6136.

506. Boekhorst, J., Helmer, Q., Kleerebezem, M., Siezen, R. J. (2006) Comparative analysis of proteins with a mucus-binding domain found exclusively in lactic acid bacteria. Microbiology 152, 273-280.

507. Rokhsefat, S., Lin, A., Comelli, E. M. (2016) Mucin-Microbiota Interaction During Postnatal Maturation of the Intestinal Ecosystem: Clinical Implications. Digestive Diseases and Sciences, 1-14.

508. Hetz, C. (2012) The unfolded protein response: controlling cell fate decisions under ER stress and beyond. Nature Reviews Molecular Cell Biology 13, 89102.

509. Heazlewood, C. K., Cook, M. C., Eri, R., Price, G. R., Tauro, S. B., Taupin, D., Thornton, D. J., Png, C. W., Crockford, T. L., Cornall, R. J. (2008) Aberrant mucin assembly in mice causes endoplasmic reticulum stress and spontaneous inflammation resembling ulcerative colitis. PLoS Medicine 5, e54.

510. Rakoff-Nahoum, S., Paglino, J., Eslami-Varzaneh, F., Edberg, S., Medzhitov, R. (2004) Recognition of commensal microflora by toll-like receptors is required for intestinal homeostasis. Cell 118, 229-241.

511. Nenci, A., Becker, C., Wullaert, A., Gareus, R., van Loo, G., Danese, S., Huth, M., Nikolaev, A., Neufert, C., Madison, B. (2007) Epithelial NEMO links innate immunity to chronic intestinal inflammation. Nature 446, 557-561.

512. Esplin, B. L., Shimazu, T., Welner, R. S., Garrett, K. P., Nie, L., Zhang, Q., Humphrey, M. B., Yang, Q., Borghesi, L. A., Kincade, P. W. (2011) Chronic 
exposure to a TLR ligand injures hematopoietic stem cells. The Journal of Immunology 186, 5367-5375.

513. Beerman, I., Bhattacharya, D., Zandi, S., Sigvardsson, M., Weissman, I. L., Bryder, D., Rossi, D. J. (2010) Functionally distinct hematopoietic stem cells modulate hematopoietic lineage potential during aging by a mechanism of clonal expansion. Proceedings of the National Academy of Sciences 107, 5465-5470.

514. Ball, Z. B., Barnes, R. H., Visscher, M. B. (1947) The effects of dietary caloric restriction on maturity and senescence, with particular reference to fertility and longevity. American Journal of Physiology 150, 511-519.

515. Vermeij, W. P., Hoeijmakers, J. H., Pothof, J. (2014) Aging: not all DNA damage is equal. Current Opinion in Genetics \& Development 26, 124-130.

516. Sherwood, E. M., Blomberg, B. B., Xu, W., Warner, C. A., Riley, R. L. (1998) Cutting edge: Senescent BALB/C mice exhibit decreased expression of $\lambda 5$ surrogate light chains and reduced development within the pre-B cell compartment. The Journal of Immunology 161, 4472-4475.

517. Min, H., Montecino-Rodriguez, E., Dorshkind, K. (2004) Reduction in the developmental potential of intrathymic $T$ cell progenitors with age. The Journal of Immunology 173, 245-250.

518. Nikolich-Žugich, J. (2014) Aging of the T cell compartment in mice and humans: from no naive expectations to foggy memories. The Journal of Immunology 193, 2622-2629.

519. Kilpatrick, R. D., Rickabaugh, T., Hultin, L. E., Hultin, P., Hausner, M. A., Detels, R., Phair, J., Jamieson, B. D. (2008) Homeostasis of the naive CD4+ T cell compartment during aging. The Journal of Immunology 180, 1499-1507.

520. Yu, Z. and Morrison, M. (2004) Improved extraction of PCR-quality community DNA from digesta and fecal samples. Biotechniques 36, 808-813.

521. van den Bogert, B., Erkus, O., Boekhorst, J., de Goffau, M., Smid, E. J., Zoetendal, E. G., Kleerebezem, M. (2013) Diversity of human small intestinal Streptococcus and Veillonella populations. FEMS Microbiology Ecology 85, 376-388.

522. Caporaso, J. G., Kuczynski, J., Stombaugh, J., Bittinger, K., Bushman, F. D., Costello, E. K., Fierer, N., Pena, A. G., Goodrich, J. K., Gordon, J. I. (2010) QIIME allows analysis of high-throughput community sequencing data. Nature Methods 7, 335-336.

523. Zelante, T., lannitti, R. G., Cunha, C., De Luca, A., Giovannini, G., Pieraccini, G., Zecchi, R., D’Angelo, C., Massi-Benedetti, C., Fallarino, F. (2013) Tryptophan catabolites from microbiota engage aryl hydrocarbon receptor and balance mucosal reactivity via interleukin-22. Immunity 39, 372-385.

524. Faith, D. P. and Baker, A. M. (2006) Phylogenetic diversity (PD) and biodiversity conservation: some bioinformatics challenges. Evolutionary Bioinformatics Online 2, 121-128.

525. Herndler-Brandstetter, D., Landgraf, K., Tzankov, A., Jenewein, B., Brunauer, R., Laschober, G. T., Parson, W., Kloss, F., Gassner, R., Lepperdinger, G. (2012) The impact of aging on memory $T$ cell phenotype and function in the human bone marrow. Journal of Leukocyte Biology 91, 197-205.

526. Fessler, J., Ficjan, A., Duftner, C., Dejaco, C. (2013) The impact of aging on regulatory T-cells. Frontiers in Immunology 4.

527. Caro, P., Gómez, J., López-Torres, M., Sánchez, I., Naudí, A., Jove, M., Pamplona, R., Barja, G. (2008) Forty percent and eighty percent methionine 
restriction decrease mitochondrial ROS generation and oxidative stress in rat liver. Biogerontology 9, 183-196.

528. Sanz, A., Caro, P., Ayala, V., Portero-Otin, M., Pamplona, R., Barja, G. (2006) Methionine restriction decreases mitochondrial oxygen radical generation and leak as well as oxidative damage to mitochondrial DNA and proteins. The FASEB Journal 20, 1064-1073.

529. Hasek, B. E., Stewart, L. K., Henagan, T. M., Boudreau, A., Lenard, N. R., Black, C., Shin, J., Huypens, P., Malloy, V. L., Plaisance, E. P. (2010) Dietary methionine restriction enhances metabolic flexibility and increases uncoupled respiration in both fed and fasted states. American Journal of PhysiologyRegulatory, Integrative and Comparative Physiology 299, R728-R739.

530. Yokota, T., Meka, C. R., Kouro, T., Medina, K. L., Igarashi, H., Takahashi, M., Oritani, K., Funahashi, T., Tomiyama, Y., Matsuzawa, Y. (2003) Adiponectin, a fat cell product, influences the earliest lymphocyte precursors in bone marrow cultures by activation of the cyclooxygenase-prostaglandin pathway in stromal cells. The Journal of Immunology 171, 5091-5099.

531. Yoshino, J., Mills, K. F., Yoon, M. J., Imai, S.-i. (2011) Nicotinamide mononucleotide, a key NAD+ intermediate, treats the pathophysiology of dietand age-induced diabetes in mice. Cell Metabolism 14, 528-536.

532. Allman, D. and Miller, J. P. (2005) The aging of early B-cell precursors. Immunological Reviews 205, 18-29.

533. Rongvaux, A., Shea, R. J., Mulks, M. H., Gigot, D., Urbain, J., Leo, O., Andris, F. (2002) Pre-B-cell colony-enhancing factor, whose expression is upregulated in activated lymphocytes, is a nicotinamide phosphoribosyltransferase, a cytosolic enzyme involved in NAD biosynthesis. European Journal of Immunology 32, 3225-3234.

534. Jia, S. H., Li, Y., Parodo, J., Kapus, A., Fan, L., Rotstein, O. D., Marshall, J. C. (2004) Pre-B cell colony-enhancing factor inhibits neutrophil apoptosis in experimental inflammation and clinical sepsis. Journal of Clinical Investigation 113, 1318-1327.

535. Samal, B., Sun, Y., Stearns, G., Xie, C., Suggs, S., McNiece, I. (1994) Cloning and characterization of the cDNA encoding a novel human pre-B-cell colonyenhancing factor. Molecular and Cellular Biology 14, 1431-1437.

536. Collado, M. C., Derrien, M., Isolauri, E., de Vos, W. M., Salminen, S. (2007) Intestinal integrity and Akkermansia muciniphila, a mucin-degrading member of the intestinal microbiota present in infants, adults, and the elderly. Applied and Environmental Microbiology 73, 7767-7770.

537. Vujkovic-Cvijin, I., Dunham, R. M., Iwai, S., Maher, M. C., Albright, R. G., Broadhurst, M. J., Hernandez, R. D., Lederman, M. M., Huang, Y., Somsouk, M. (2013) Dysbiosis of the gut microbiota is associated with HIV disease progression and tryptophan catabolism. Science Translational Medicine 5, 193ra91-193ra91.

538. Frank, D. N., Amand, A. L. S., Feldman, R. A., Boedeker, E. C., Harpaz, N., Pace, N. R. (2007) Molecular-phylogenetic characterization of microbial community imbalances in human inflammatory bowel diseases. Proceedings of the National Academy of Sciences 104, 13780-13785.

539. Wolf, A. M., Wolf, D., Rumpold, H., Moschen, A. R., Kaser, A., Obrist, P., Fuchs, D., Brandacher, G., Winkler, C., Geboes, K. (2004) Overexpression of indoleamine 2, 3-dioxygenase in human inflammatory bowel disease. Clinical Immunology 113, 47-55. 
540. Watsuji, T.-o., Takano, H., Yamabe, T., Tamazawa, S., Ikemura, H., Ohishi, T., Matsuda, T., Shiratori-Takano, H., Beppu, T., Ueda, K. (2014) Analysis of the tryptophanase expression in Symbiobacterium thermophilum in a coculture with Geobacillus stearothermophilus. Applied Microbiology and Biotechnology 98, 10177-10186.

541. Zhang, C., Yin, A., Li, H., Wang, R., Wu, G., Shen, J., Zhang, M., Wang, L., Hou, Y., Ouyang, H. (2015) Dietary Modulation of Gut Microbiota Contributes to Alleviation of Both Genetic and Simple Obesity in Children. EBioMedicine 2, 966-982.

542. Hooper, L. V., Littman, D. R., Macpherson, A. J. (2012) Interactions between the microbiota and the immune system. Science 336, 1268-1273.

543. Eckburg, P. B., Bik, E. M., Bernstein, C. N., Purdom, E., Dethlefsen, L., Sargent, M., Gill, S. R., Nelson, K. E., Relman, D. A. (2005) Diversity of the human intestinal microbial flora. science 308, 1635-1638.

544. Leser, T. D., Amenuvor, J. Z., Jensen, T. K., Lindecrona, R. H., Boye, M., Møller, K. (2002) Culture-independent analysis of gut bacteria: the pig gastrointestinal tract microbiota revisited. Applied and Environmental Microbiology 68, 673-690.

545. Ley, R. E., Bäckhed, F., Turnbaugh, P., Lozupone, C. A., Knight, R. D., Gordon, J. I. (2005) Obesity alters gut microbial ecology. Proceedings of the National Academy of Sciences of the United States of America 102, 1107011075.

546. Rescigno, M. (2014) Intestinal microbiota and its effects on the immune system. Cellular Microbiology 16, 1004-1013.

547. Carding, S., Verbeke, K., Vipond, D. T., Corfe, B. M., Owen, L. (2015) Dysbiosis of the gut microbiota in disease. Microbial Ecology in Health and Disease 26.

548. Costello, E. K., Lauber, C. L., Hamady, M., Fierer, N., Gordon, J. I., Knight, R. (2009) Bacterial community variation in human body habitats across space and time. Science 326, 1694-1697.

549. Claesson, M. J., Cusack, S., O'Sullivan, O., Greene-Diniz, R., de Weerd, H., Flannery, E., Marchesi, J. R., Falush, D., Dinan, T., Fitzgerald, G. (2011) Composition, variability, and temporal stability of the intestinal microbiota of the elderly. Proceedings of the National Academy of Sciences 108, 45864591.

550. Mariat, D., Firmesse, O., Levenez, F., Guimarăes, V., Sokol, H., Dore, J., Corthier, G., Furet, J. (2009) The Firmicutes/Bacteroidetes ratio of the human microbiota changes with age. BMC Microbiology 9.

551. Hopkins, M., Sharp, R., Macfarlane, G. (2001) Age and disease related changes in intestinal bacterial populations assessed by cell culture, 16S rRNA abundance, and community cellular fatty acid profiles. Gut 48, 198-205.

552. Mueller, S., Saunier, K., Hanisch, C., Norin, E., Alm, L., Midtvedt, T., Cresci, A., Silvi, S., Orpianesi, C., Verdenelli, M. C. (2006) Differences in fecal microbiota in different European study populations in relation to age, gender, and country: a cross-sectional study. Applied and Environmental Microbiology 72, 1027-1033.

553. Miquel, S., Martin, R., Rossi, O., Bermudez-Humaran, L., Chatel, J., Sokol, H., Thomas, M., Wells, J., Langella, P. (2013) Faecalibacterium prausnitzii and human intestinal health. Current Opinion in Microbiology 16, 255-261.

554. Franceschi, C., Capri, M., Monti, D., Giunta, S., Olivieri, F., Sevini, F., Panourgia, M. P., Invidia, L., Celani, L., Scurti, M. (2007) Inflammaging and 
anti-inflammaging: a systemic perspective on aging and longevity emerged from studies in humans. Mechanisms of Ageing and Development 128, 92105.

555. Cesari, M., Penninx, B. W., Newman, A. B., Kritchevsky, S. B., Nicklas, B. J., Sutton-Tyrrell, K., Rubin, S. M., Ding, J., Simonsick, E. M., Harris, T. B. (2003) Inflammatory markers and onset of cardiovascular events results from the Health ABC Study. Circulation 108, 2317-2322.

556. Forlenza, O. V., Diniz, B. S., Talib, L. L., Mendonça, V. A., Ojopi, E. B., Gattaz, W. F., Teixeira, A. L. (2009) Increased serum IL-1 $\beta$ level in Alzheimer's disease and mild cognitive impairment. Dementia and Geriatric Cognitive Disorders 28, 507-512.

557. Whiteley, W., Jackson, C., Lewis, S., Lowe, G., Rumley, A., Sandercock, P., Wardlaw, J., Dennis, M., Sudlow, C. (2009) Inflammatory markers and poor outcome after stroke: a prospective cohort study and systematic review of interleukin-6. PLoS Medicine 6, e1000145.

558. Mullin, J., Valenzano, M., Verrecchio, J., Kothari, R. (2002) Age-and dietrelated increase in transepithelial colon permeability of Fischer 344 rats. Digestive Diseases and Sciences 47, 2262-2270.

559. Tran, L. and Greenwood-Van Meerveld, B. (2013) Age-associated remodeling of the intestinal epithelial barrier. The Journals of Gerontology Series A: Biological Sciences and Medical Sciences 68, 1045-1056.

560. Rehman, T. (2012) Role of the gut microbiota in age-related chronic inflammation. Endocrine, Metabolic \& Immune Disorders-Drug Targets (Formerly Current Drug Targets-Immune, Endocrine \& Metabolic Disorders) 12, 361-367.

561. Gentleman, R. C., Carey, V. J., Bates, D. M., Bolstad, B., Dettling, M., Dudoit, S., Ellis, B., Gautier, L., Ge, Y., Gentry, J. (2004) Bioconductor: open software development for computational biology and bioinformatics. Genome Biology 5, 1.

562. Lin, K., Kools, H., de Groot, P. J., Gavai, A. K., Basnet, R. K., Cheng, F., Wu, J., Wang, X., Lommen, A., Hooiveld, G. (2011) MADMAX-Management and analysis database for multiple omics experiments. Journal of Integrative Bioinformatics 8, 160.

563. Dai, M., Wang, P., Boyd, A. D., Kostov, G., Athey, B., Jones, E. G., Bunney, W. E., Myers, R. M., Speed, T. P., Akil, H. (2005) Evolving gene/transcript definitions significantly alter the interpretation of GeneChip data. Nucleic Acids Research 33, e175-e175.

564. Bolstad, B., Collin, F., Simpson, K., Irizarry, R., Speed, T. (2004) Experimental design and low-level analysis of microarray data. International Review of Neurobiology 60, 25-58.

565. Berry, D., Mahfoudh, K. B., Wagner, M., Loy, A. (2011) Barcoded primers used in multiplex amplicon pyrosequencing bias amplification. Applied and Environmental Microbiology 77, 7846-7849.

566. van den Bogert, B., de Vos, W. M., Zoetendal, E. G., Kleerebezem, M. (2011) Microarray analysis and barcoded pyrosequencing provide consistent microbial profiles depending on the source of human intestinal samples. Applied and Environmental Microbiology 77, 2071-2080.

567. Quast, C., Pruesse, E., Yilmaz, P., Gerken, J., Schweer, T., Yarza, P., Peplies, J., Glöckner, F. O. (2013) The SILVA ribosomal RNA gene database project: improved data processing and web-based tools. Nucleic Acids Research 41, D590-D596. 
568. Storey, J. D. and Tibshirani, R. (2003) Statistical significance for genomewide studies. Proceedings of the National Academy of Sciences 100, 9440-9445.

569. Sartor, M. A., Tomlinson, C. R., Wesselkamper, S. C., Sivaganesan, S., Leikauf, G. D., Medvedovic, M. (2006) Intensity-based hierarchical Bayes method improves testing for differentially expressed genes in microarray experiments. BMC Bioinformatics 7.

570. Ainsworth, T. D., Krause, L., Bridge, T., Torda, G., Raina, J.-B., Zakrzewski, M., Gates, R. D., Padilla-Gamiño, J. L., Spalding, H. L., Smith, C. (2015) The coral core microbiome identifies rare bacterial taxa as ubiquitous endosymbionts. The ISME Journal 9, 2261-2274.

571. Jensen, L. J., Kuhn, M., Stark, M., Chaffron, S., Creevey, C., Muller, J., Doerks, T., Julien, P., Roth, A., Simonovic, M. (2009) STRING 8-a global view on proteins and their functional interactions in 630 organisms. Nucleic Acids Research 37, D412-D416.

572. Cani, P. D., Amar, J., Iglesias, M. A., Poggi, M., Knauf, C., Bastelica, D., Neyrinck, A. M., Fava, F., Tuohy, K. M., Chabo, C. (2007) Metabolic endotoxemia initiates obesity and insulin resistance. Diabetes 56, 1761-1772.

573. Cani, P. D., Bibiloni, R., Knauf, C., Waget, A., Neyrinck, A. M., Delzenne, N. M., Burcelin, R. (2008) Changes in gut microbiota control metabolic endotoxemia-induced inflammation in high-fat diet-induced obesity and diabetes in mice. Diabetes 57, 1470-1481.

574. Festi, D., Schiumerini, R., Eusebi, L. H., Marasco, G., Taddia, M., Colecchia, A. (2014) Gut microbiota and metabolic syndrome. World Journal of Gastroenterology 20, 16079-16094.

575. Laukoetter, M. G., Nava, P., Nusrat, A. (2008) Role of the intestinal barrier in inflammatory bowel disease. World Journal of Gastroenterology 14, 401-407.

576. Ivanov, I. I., Atarashi, K., Manel, N., Brodie, E. L., Shima, T., Karaoz, U., Wei, D., Goldfarb, K. C., Santee, C. A., Lynch, S. V. (2009) Induction of intestinal Th17 cells by segmented filamentous bacteria. Cell 139, 485-498.

577. Mazmanian, S. K., Liu, C. H., Tzianabos, A. O., Kasper, D. L. (2005) An immunomodulatory molecule of symbiotic bacteria directs maturation of the host immune system. Cell 122, 107-118.

578. Mazmanian, S. K., Round, J. L., Kasper, D. L. (2008) A microbial symbiosis factor prevents intestinal inflammatory disease. Nature 453, 620-625.

579. Round, J. L., Lee, S. M., Li, J., Tran, G., Jabri, B., Chatila, T. A., Mazmanian, S. K. (2011) The Toll-like receptor 2 pathway establishes colonization by a commensal of the human microbiota. Science 332, 974-977.

580. Atarashi, K., Tanoue, T., Oshima, K., Suda, W., Nagano, Y., Nishikawa, H., Fukuda, S., Saito, T., Narushima, S., Hase, K. (2013) Treg induction by a rationally selected mixture of Clostridia strains from the human microbiota. Nature 500, 232-236.

581. Atarashi, K., Tanoue, T., Shima, T., Imaoka, A., Kuwahara, T., Momose, Y., Cheng, G., Yamasaki, S., Saito, T., Ohba, Y. (2011) Induction of colonic regulatory $T$ cells by indigenous Clostridium species. Science 331, 337-341.

582. Bradley, J. (2008) TNF-mediated inflammatory disease. The Journal of pathology 214, 149-160.

583. Peyrin-Biroulet, L. (2010) Anti-TNF therapy in inflammatory bowel diseases: a huge review. Minerva Gastroenterologica e Dietologica 56, 233-243.

584. Ma, T. Y., Iwamoto, G. K., Hoa, N. T., Akotia, V., Pedram, A., Boivin, M. A., Said, H. M. (2004) TNF-a-induced increase in intestinal epithelial tight junction 
permeability requires NF-kB activation. American Journal of PhysiologyGastrointestinal and Liver Physiology 286, G367-G376.

585. Wesemann, D. R., Portuguese, A. J., Meyers, R. M., Gallagher, M. P., CluffJones, K., Magee, J. M., Panchakshari, R. A., Rodig, S. J., Kepler, T. B., Alt, F. W. (2013) Microbial colonization influences early B-lineage development in the gut lamina propria. Nature 501, 112-115.

586. Hertz, M. and Nemazee, D. (1997) BCR ligation induces receptor editing in IgM+ IgD- bone marrow B cells in vitro. Immunity 6, 429-436.

587. Retter, M. W. and Nemazee, D. (1998) Receptor editing occurs frequently during normal B cell development. The Journal of Experimental Medicine 188, 1231-1238.

588. Tiegs, S. L., Russell, D. M., Nemazee, D. (1993) Receptor editing in selfreactive bone marrow B cells. The Journal of Experimental Medicine 177, 1009-1020.

589. Everard, A., Belzer, C., Geurts, L., Ouwerkerk, J. P., Druart, C., Bindels, L. B., Guiot, Y., Derrien, M., Muccioli, G. G., Delzenne, N. M. (2013) Cross-talk between Akkermansia muciniphila and intestinal epithelium controls dietinduced obesity. Proceedings of the National Academy of Sciences 110, 9066-9071.

590. Langille, M. G., Meehan, C. J., Koenig, J. E., Dhanani, A. S., Rose, R. A., Howlett, S. E., Beiko, R. G. (2014) Microbial shifts in the aging mouse gut. Microbiome 2, 50.

591. Wang, F., Yu, T., Huang, G., Cai, D., Liang, X., Su, H., Zhu, Z., Li, D., Yang, Y., Shen, P. (2015) Gut microbiota community and its assembly associated with age and diet in Chinese centenarians. Journal of Microbioly Biotechnology 25, 1195-204.

592. Belkaid, Y. and Hand, T. W. (2014) Role of the microbiota in immunity and inflammation. Cell 157, 121-141.

593. Fagarasan, S., Muramatsu, M., Suzuki, K., Nagaoka, H., Hiai, H., Honjo, T. (2002) Critical roles of activation-induced cytidine deaminase in the homeostasis of gut flora. Science 298, 1424-1427.

594. Fransen, F., Zagato, E., Mazzini, E., Fosso, B., Manzari, C., El Aidy, S., Chiavelli, A., D’Erchia, A. M., Sethi, M. K., Pabst, O. (2015) BALB/c and C57BL/6 mice differ in polyreactive $\lg A$ abundance, which impacts the generation of antigen-specific $\lg A$ and microbiota diversity. Immunity 43, 527540.

595. Suzuki, K., Meek, B., Doi, Y., Muramatsu, M., Chiba, T., Honjo, T., Fagarasan, S. (2004) Aberrant expansion of segmented filamentous bacteria in $\lg A$ deficient gut. Proceedings of the National Academy of Sciences 101, 19811986.

596. Jakobsson, H. E., Rodríguez-Piñeiro, A. M., Schütte, A., Ermund, A., Boysen, P., Bemark, M., Sommer, F., Bäckhed, F., Hansson, G. C., Johansson, M. E. (2015) The composition of the gut microbiota shapes the colon mucus barrier. EMBO Reports 16, 164-177.

597. Kuehbacher, T., Rehman, A., Lepage, P., Hellmig, S., Fölsch, U. R., Schreiber, S., Ott, S. J. (2008) Intestinal TM7 bacterial phylogenies in active inflammatory bowel disease. Journal of Medical Microbiology 57, 1569-1576.

598. Mukhopadhya, I., Hansen, R., El-Omar, E. M., Hold, G. L. (2012) IBD-what role do Proteobacteria play? Nature Reviews Gastroenterology and Hepatology 9, 219-230. 
599. Rowan, F., Docherty, N. G., Murphy, M., Murphy, B., Coffey, J. C., O‘Connell, P. R. (2010) Desulfovibrio bacterial species are increased in ulcerative colitis. Diseases of the Colon \& Rectum 53, 1530-1536.

600. Biagi, E., Candela, M., Fairweather-Tait, S., Franceschi, C., Brigidi, P. (2012) Ageing of the human metaorganism: the microbial counterpart. Age 34, 247267.

601. Desai, A., Grolleau-Julius, A., Yung, R. (2010) Leukocyte function in the aging immune system. Journal of Leukocyte Biology 87, 1001-1009.

602. Busse, P. J., Zhang, T. F., Srivastava, K., Schofield, B., Li, X. M. (2007) Effect of ageing on pulmonary inflammation, airway hyperresponsiveness and $T$ and $B$ cell responses in antigen-sensitized and-challenged mice. Clinical \& Experimental Allergy 37, 1392-1403.

603. Agrawal, A., Agrawal, S., Cao, J. N., Su, H., Osann, K., Gupta, S. (2007) Altered innate immune functioning of dendritic cells in elderly humans: a role of phosphoinositide 3-kinase-signaling pathway. The Journal of Immunology 178, 6912-6922.

604. Agrawal, A., Agrawal, S., Tay, J., Gupta, S. (2008) Biology of dendritic cells in aging. Journal of Clinical Immunology 28, 14-20.

605. Shodell, M. and Siegal, F. (2002) Circulating, Interferon Producing Plasmacytoid Dendritic Cells Decline During Human Ageing. Scandinavian Journal of Immunology 56, 518-521.

606. Jing, Y., Shaheen, E., Drake, R. R., Chen, N., Gravenstein, S., Deng, Y. (2009) Aging is associated with a numerical and functional decline in plasmacytoid dendritic cells, whereas myeloid dendritic cells are relatively unaltered in human peripheral blood. Human Immunology 70, 777-784.

607. Pérez-Cabezas, B., Naranjo-Gómez, M., Fernández, M. A., Grífols, J. R., Pujol-Borrell, R., Borr s, F. E. (2007) Reduced numbers of plasmacytoid dendritic cells in aged blood donors. Experimental Gerontology 42, 10331038.

608. Wong, C. P., Magnusson, K. R., Ho, E. (2010) Aging is associated with altered dendritic cells subset distribution and impaired proinflammatory cytokine production. Experimental Gerontology 45, 163-169.

609. Grolleau-Julius, A., Harning, E. K., Abernathy, L. M., Yung, R. L. (2008) Impaired dendritic cell function in aging leads to defective antitumor immunity. Cancer Research 68, 6341-6349.

610. Grolleau-Julius, A., Garg, M. R., Mo, R. R., Stoolman, L. L., Yung, R. L. (2006) Effect of Aging on Bone Marrow-Derived Murine CD11C+ CD4- CD8 Dendritic Cell Function. The Journals of Gerontology Series A: Biological Sciences and Medical Sciences 61, 1039-1047.

611. Wong, C. and Goldstein, D. R. (2013) Impact of aging on antigen presentation cell function of dendritic cells. Current Opinion in Immunology 25, 535-541.

612. Geissmann, F., Manz, M. G., Jung, S., Sieweke, M. H., Merad, M., Ley, K. (2010) Development of monocytes, macrophages, and dendritic cells. Science 327, 656-661.

613. Biswas, S. K. and Mantovani, A. (2012) Orchestration of metabolism by macrophages. Cell Metabolism 15, 432-437.

614. Hannum, G., Guinney, J., Zhao, L., Zhang, L., Hughes, G., Sadda, S., Klotzle, B., Bibikova, M., Fan, J.-B., Gao, Y. (2013) Genome-wide methylation profiles reveal quantitative views of human aging rates. Molecular Cell 49, 359-367. 
615. Cho, S.-H., Chen, J. A., Sayed, F., Ward, M. E., Gao, F., Nguyen, T. A., Krabbe, G., Sohn, P. D., Lo, I., Minami, S. (2015) SIRT1 deficiency in microglia contributes to cognitive decline in aging and neurodegeneration via epigenetic regulation of IL-1 $\beta$. The Journal of Neuroscience 35, 807-818.

616. Li, P., Spann, N. J., Kaikkonen, M. U., Lu, M., Fox, J. N., Bandyopadhyay, G., Talukdar, S., Xu, J., Lagakos, W. S., Patsouris, D. (2013) NCoR repression of LXRs restricts macrophage biosynthesis of insulin-sensitizing omega 3 fatty acids. Cell 155, 200-214.

617. Remely, M., Aumueller, E., Merold, C., Dworzak, S., Hippe, B., Zanner, J., Pointner, A., Brath, H., Haslberger, A. G. (2014) Effects of short chain fatty acid producing bacteria on epigenetic regulation of FFAR3 in type 2 diabetes and obesity. Gene 537, 85-92.

618. Horvath, S. (2013) DNA methylation age of human tissues and cell types. Genome Biology 14.

619. Jones, M. J., Goodman, S. J., Kobor, M. S. (2015) DNA methylation and healthy human aging. Aging Cell 14, 924-932.

620. Sharma, P., Tomar, S. K., Goswami, P., Sangwan, V., Singh, R. (2014) Antibiotic resistance among commercially available probiotics. Food Research International 57, 176-195.

621. Besselink, M. G., van Santvoort, H. C., Buskens, E., Boermeester, M. A., van Goor, H., Timmerman, H. M., Nieuwenhuijs, V. B., Bollen, T. L., van Ramshorst, B., Witteman, B. J. (2008) Probiotic prophylaxis in predicted severe acute pancreatitis: a randomised, double-blind, placebo-controlled trial. The Lancet 371, 651-659.

622. Ridwan, B., Koning, C., Besselink, M., Timmerman, H., Brouwer, E., Verhoef, J., Gooszen, H., Akkermans, L. (2008) Antimicrobial activity of a multispecies probiotic (Ecologic 641) against pathogens isolated from infected pancreatic necrosis. Letters in Applied Microbiology 46, 61-67.

623. Timmerman, H. M., Niers, L. E., Ridwan, B. U., Koning, C. J., Mulder, L., Akkermans, L. M., Rombouts, F. M., Rijkers, G. T. (2007) Design of a multispecies probiotic mixture to prevent infectious complications in critically ill patients. Clinical Nutrition 26, 450-459.

624. Bos, N., Meeuwsen, C., Hooijkaas, H., Benner, R., Wostmann, B., Pleasants, J. (1987) Early development of lg-secreting cells in young of germ-free BALB/c mice fed a chemically defined ultrafiltered diet. Cellular Immunology 105, 235-245.

625. He, B., Xu, W., Santini, P. A., Polydorides, A. D., Chiu, A., Estrella, J., Shan, M., Chadburn, A., Villanacci, V., Plebani, A. (2007) Intestinal bacteria trigger T cell-independent immunoglobulin A 2 class switching by inducing epithelialcell secretion of the cytokine APRIL. Immunity 26, 812-826.

626. Hapfelmeier, S., Lawson, M. A., Slack, E., Kirundi, J. K., Stoel, M., Heikenwalder, M., Cahenzli, J., Velykoredko, Y., Balmer, M. L., Endt, K., Geuking, M. B., Curtiss, R., McCoy, K. D., Macpherson, A. J. (2010) Reversible microbial colonization of germ-free mice reveals the dynamics of IgA immune responses. Science 328, 1705-1709.

627. Geuking, M. B., Cahenzli, J., Lawson, M. A., Ng, D. C., Slack, E., Hapfelmeier, S., McCoy, K. D., Macpherson, A. J. (2011) Intestinal bacterial colonization induces mutualistic regulatory $\mathrm{T}$ cell responses. Immunity 34 , 794-806.

628. Hall, J. A., Bouladoux, N., Sun, C. M., Wohlfert, E. A., Blank, R. B., Zhu, Q., Grigg, M. E., Berzofsky, J. A., Belkaid, Y. (2008) Commensal DNA limits 
regulatory $\mathrm{T}$ cell conversion and is a natural adjuvant of intestinal immune responses. Immunity 29, 637-649.

629. Hrncir, T., Stepankova, R., Kozakova, H., Hudcovic, T., Tlaskalova-Hogenova, $\mathrm{H}$. (2008) Gut microbiota and lipopolysaccharide content of the diet influence development of regulatory $T$ cells: studies in germ-free mice. BMC Immunology 9, 65.

630. Caramalho, I., Lopes-Carvalho, T., Ostler, D., Zelenay, S., Haury, M., Demengeot, J. (2003) Regulatory T cells selectively express toll-like receptors and are activated by lipopolysaccharide. The Journal of Experimental Medicine 197, 403-411.

631. Maldonado, L., Lammers, A., Nieuwland, M., Reilingh, G. D. V., Parmentier, H. (2005) Homotopes affect primary and secondary antibody responses in poultry. Vaccine 23, 2731-2739.

632. Round, J. L. and Mazmanian, S. K. (2009) The gut microbiota shapes intestinal immune responses during health and disease. Nature Reviews Immunology 9, 313-323.

633. Conley, M. N., Wong, C. P., Duyck, K. M., Hord, N., Ho, E., Sharpton, T. J. (2016) Aging and serum MCP-1 are associated with gut microbiome composition in a murine model. PeerJ 4, e1854.

634. Wang, J., Li, F., Sun, R., Gao, X., Wei, H., Li, L.-J., Tian, Z. (2013) Bacterial colonization dampens influenza-mediated acute lung injury via induction of M2 alveolar macrophages. Nature Communications 4, 2106.

635. Hayashi, E. A., Akira, S., Nobrega, A. (2005) Role of TLR in B cell development: signaling through TLR4 promotes $B$ cell maturation and is inhibited by TLR2. The Journal of Immunology 174, 6639-6647.

636. Azulay-Debby, H., Edry, E., Melamed, D. (2007) CpG DNA stimulates autoreactive immature $B$ cells in the bone marrow. European Journal of Immunology 37, 1463-1475.

637. Edry, E., Azulay-Debby, H., Melamed, D. (2008) TOLL-like receptor ligands stimulate aberrant class switch recombination in early B cell precursors. International Immunology 20, 1575-1585.

638. Hayashi, E. A., Granato, A., Paiva, L. S., Bertho, Á. L., Bellio, M., Nobrega, A. (2010) TLR4 promotes $B$ cell maturation: independence and cooperation with B lymphocyte-activating factor. The Journal of Immunology 184, 4662-4672.

639. Li, Q., Han, D., Wang, W., Liu, X., Sun, X., Zhang, J., Li, R., Zhang, Y. (2014) Toll-like receptor 4-mediated signaling regulates IL-7-driven proliferation and differentiation of B-cell precursors. Cellular \& Molecular Immunology 11, 132140.

640. Khosravi, A., Yáñez, A., Price, J. G., Chow, A., Merad, M., Goodridge, H. S., Mazmanian, S. K. (2014) Gut microbiota promote hematopoiesis to control bacterial infection. Cell Host \& Microbe 15, 374-381.

641. Joyce, S. A., MacSharry, J., Casey, P. G., Kinsella, M., Murphy, E. F., Shanahan, F., Hill, C., Gahan, C. G. (2014) Regulation of host weight gain and lipid metabolism by bacterial bile acid modification in the gut. Proceedings of the National Academy of Sciences 111, 7421-7426.

642. Bäckhed, F., Ding, H., Wang, T., Hooper, L. V., Koh, G. Y., Nagy, A., Semenkovich, C. F., Gordon, J. I. (2004) The gut microbiota as an environmental factor that regulates fat storage. Proceedings of the National Academy of Sciences of the United States of America 101, 15718-15723. 
643. Umemoto, Y., Tsuji, K., Yang, F.-C., Ebihara, Y., Kaneko, A., Furukawa, S., Nakahata, T. (1997) Leptin stimulates the proliferation of murine myelocytic and primitive hematopoietic progenitor cells. Blood 90, 3438-3443.

644. Fujita, Y., Yanagida, H., Mimori, T., Jin, Z.-X., Sakai, T., Kawanami, T., Sawaki, T., Masaki, Y., Fukushima, T., Okazaki, T. (2012) Prevention of fasting-mediated bone marrow atrophy by leptin administration. Cellular Immunology 273, 52-58.

645. Stephan, R. P., Lill-Elghanian, D. A., Witte, P. L. (1997) Development of B cells in aged mice: decline in the ability of pro-B cells to respond to IL-7 but not to other growth factors. The Journal of Immunology 158, 1598-1609.

646. Stephan, R. P., Reilly, C. R., Witte, P. L. (1998) Impaired ability of bone marrow stromal cells to support B-lymphopoiesis with age. Blood 91, 75-88.

647. Clark, M. R., Mandal, M., Ochiai, K., Singh, H. (2014) Orchestrating B cell lymphopoiesis through interplay of IL-7 receptor and pre-B cell receptor signalling. Nature Reviews Immunology 14, 69-80.

648. Nagasawa, T. (2006) Microenvironmental niches in the bone marrow required for B-cell development. Nature Reviews Immunology 6, 107-116.

649. Bilwani, F. A. and Knight, K. L. (2012) Adipocyte-derived soluble factor (s) inhibits early stages of B lymphopoiesis. The Journal of Immunology 189, 4379-4386.

650. Kennedy, D. E. and Knight, K. L. (2015) Inhibition of B lymphopoiesis by adipocytes and IL-1-producing myeloid-derived suppressor cells. The Journal of Immunology 195, 2666-2674.

651. Justesen, J., Stenderup, K., Ebbesen, E., Mosekilde, L., Steiniche, T., Kassem, M. (2001) Adipocyte tissue volume in bone marrow is increased with aging and in patients with osteoporosis. Biogerontology 2, 165-171.

652. Poehls, J., Wassel, C., Harris, T. B., Havel, P. J., Swarbrick, M. M., Cummings, S. R., Newman, A. B., Satterfield, S., Kanaya, A. M. (2009) Association of adiponectin with mortality in older adults: the Health, Aging, and Body Composition Study. Diabetologia 52, 591-595.

653. Baylis, D., Bartlett, D., Syddall, H., Ntani, G., Gale, C., Cooper, C., Lord, J., Sayer, A. (2013) Immune-endocrine biomarkers as predictors of frailty and mortality: a 10-year longitudinal study in community-dwelling older people. Age 35, 963-971.

654. Athman, R. and Philpott, D. (2004) Innate immunity via Toll-like receptors and Nod proteins. Current Opinion in Microbiology 7, 25-32.

655. Jaronen, M. and Quintana, F. J. (2014) Immunological relevance of the coevolution of IDO1 and AHR. Frontiers in Immunology 5, 521.

656. Thurmond, T. S., Staples, J. E., Silverstone, A. E., Gasiewicz, T. A. (2000) The aryl hydrocarbon receptor has a role in the in vivo maturation of murine bone marrow $\mathrm{B}$ lymphocytes and their response to 2, 3, 7, 8tetrachlorodibenzo-p-dioxin. Toxicology and Applied Pharmacology 165, 227236.

657. Kuhla, A., Hahn, S., Butschkau, A., Lange, S., Wree, A., Vollmar, B. (2013) Lifelong caloric restriction reprograms hepatic fat metabolism in mice. The Journals of Gerontology Series A: Biological Sciences and Medical Sciences 69, 915-922.

658. Minor, R. K., Allard, J. S., Younts, C. M., Ward, T. M., de Cabo, R. (2010) Dietary interventions to extend life span and health span based on calorie restriction. The Journals of Gerontology Series A: Biological Sciences and Medical Sciences 65A, 695-703. 
659. Spaulding, C. C., Walford, R. L., Effros, R. B. (1997) Calorie restriction inhibits the age-related dysregulation of the cytokines TNF- $\alpha$ and IL-6 in C3B10RF1 mice. Mechanisms of Ageing and Development 93, 87-94.

660. Zhang, C., Li, S., Yang, L., Huang, P., Li, W., Wang, S., Zhao, G., Zhang, M., Pang, X., Yan, Z. (2013) Structural modulation of gut microbiota in life-long calorie-restricted mice. Nature Communications 4, 2163.

661. Shushimita, S., de Bruijn, M. J., de Bruin, R. W., IJzermans, J. N., Hendriks, R. W., Dor, F. J. (2014) Dietary restriction and fasting arrest B and T cell development and increase mature $\mathrm{B}$ and $\mathrm{T}$ cell numbers in bone marrow. PLoS ONE 9, e87772.

662. Delgado, P. L., Charney, D. S., Price, L. H., Landis, H., Heninger, G. (1989) Neuroendocrine and behavioral effects of dietary tryptophan restriction in healthy subjects. Life Sciences 45, 2323-2332.

663. Richie, J. P., Leutzinger, Y., Parthasarathy, S., Malloy, V., Orentreich, N., Zimmerman, J. (1994) Methionine restriction increases blood glutathione and longevity in F344 rats. The FASEB Journal 8, 1302-1307.

664. Sun, L., Akha, A. A. S., Miller, R. A., Harper, J. M. (2009) Life-span extension in mice by preweaning food restriction and by methionine restriction in middle age. The Journals of Gerontology Series A: Biological Sciences and Medical Sciences 64A, 711-722.

665. Ruckenstuhl, C., Netzberger, C., Entfellner, I., Carmona-Gutierrez, D., Kickenweiz, T., Stekovic, S., Gleixner, C., Schmid, C., Klug, L., Sorgo, A. G. (2014) Lifespan extension by methionine restriction requires autophagydependent vacuolar acidification. PLoS Genetics 10, e1004347.

666. Lees, E. K., Król, E., Grant, L., Shearer, K., Wyse, C., Moncur, E., Bykowska, A. S., Mody, N., Gettys, T. W., Delibegovic, M. (2014) Methionine restriction restores a younger metabolic phenotype in adult mice with alterations in fibroblast growth factor 21. Aging Cell 13, 817-827.

667. Caro, P., Gomez, J., Sanchez, I., Naudi, A., Ayala, V., López-Torres, M., Pamplona, R., Barja, G. (2009) Forty percent methionine restriction decreases mitochondrial oxygen radical production and leak at complex I during forward electron flow and lowers oxidative damage to proteins and mitochondrial DNA in rat kidney and brain mitochondria. Rejuvenation Research 12, 421-434.

668. Vermeij, W., Dollé, M., Reiling, E., Jaarsma, D., Payan-Gomez, C., Bombardieri, C., Wu, H., Roks, A., Botter, S., van der Eerden, B., Youssef, S., Kuiper, R., Nagarajah, B., van Oostrom, C., Brandt, R., Barnhoorn, S., Imholz, S., Pennings, J., De Bruin, A., Gyenis, Á., Pothof, J., Vijg, J., Van Steeg, H., Hoeijmakers, J. H. (2016) Restricted diet delays accelerated ageing and genomic stress in DNA-repair-deficient mice. Nature 537, 427-431.

669. Loonen, L., Stolte, E., Jaklofsky, M., Meijerink, M., Dekker, J., van Baarlen, P., Wells, J. (2014) REG3y-deficient mice have altered mucus distribution and increased mucosal inflammatory responses to the microbiota and enteric pathogens in the ileum. Mucosal Immunology 7, 939-947.

670. Satyaraj, E. (2011) Emerging Paradigms in Immunonutrition. Topics in Companion Animal Medicine 26, 25-32. 


\section{Summary}

The increased numbers of elderly people pose a major burden to public health care and society. DNA damage is considered to be the major origin of age-related changes in the body. With aging, the immune system becomes deregulated and is characterized by a low-grade inflammation (inflammaging). In this thesis, we investigate the effects of nutritional and microbial interventions on the aging immune system.

In chapter 2, we elaborate on the role of basophils in the immune system, particularly in the initiation and perpetuation of allergic immune responses. We found that basophils and dendritic cells interact in vitro, which reciprocally affects their surface markers and cytokine production. Thus, by modulating cytokine production and surface marker expression on dendritic cells, basophils may act as accessory cells in immune responses. Because little is known about the effects of aging on basophils, we investigated in chapter 3 whether basophils are affected with aging. We found that frequencies of basophils in the spleen of aging mice are increasing, while their phenotype in bone marrow and spleen changes. Moreover, to investigate the role of microbiota in the aging process, we studied the effects of microbiota transfer from young or aged mice into germfree mice. Aging, and microbiota from aged mice, in particular affect differentiation and function of basophil precursors. These findings warrant further studies on the role of basophils in T helper-2 immune responses with aging.

The contribution of macrophages to inflammaging is described in chapter 4. Important aspects for macrophage polarization and function, like autophagy and cellular metabolism, are discussed. Targeting of aged macrophages by (nutritional) interventions may open up new therapeutic opportunities for elderly.

In chapter 5, we studied the in vitro interaction between bacterial supplementations and immune cells (whole spleen cells and macrophages). We noticed that aged immune cells mount a different response to bacterial strains than young immune cells. Based on these outcomes, we selected three bacterial strains (Lactobacillus plantarum WCFS1, Lactobacillus casei BL23, Bifidobacterium breve DSM20213) for in vivo application in chapter 6 . We used $E r c c 1^{-1 / \Delta 7}$ mice, which lack fully functional ERCC1 protein. As a consequence, DNA repair is compromised, which results in accelerated aging features in all organs, including the immune system. We supplemented $E r c c 1^{-/ \Delta 7}$ mice, as well as control $\mathrm{Ercc1}^{+/+}$mice with the three selected bacterial strains. We observed that $L$. plantarum prevented the age-related decline in mucus barrier function of $E r c c 1^{-/ \Delta 7}$ mice, whereas $B$. breve exacerbated the age-related decline in mucus barrier. L. casei supplementation elevated multiple systemic inflammatory markers in Ercc1 ${ }^{-/ \Delta 7}$ mice, including Ly6C $C^{\text {hi }}$ monocytes, neutrophils, and Th17 cells in spleen. Strikingly, we found major changes in the mucus barrier and immune system after supplementation of $\mathrm{ErCC}^{-1 \Delta 7}$ mice with $L$. plantarum and L. casei, but not after sup- 
plementation of $\mathrm{ErCC}^{+/+}$mice. Therefore, we conclude that caution is needed in the selection of candidate probiotic strains for supplementation of aging individuals.

In chapter 7, we took a different approach to modulate the aging immune system by applying dietary tryptophan restriction in $\mathrm{ErCC}^{+/+}$and $\mathrm{ErCC}^{-/ \Delta 7}$ mice. We observed that in both mouse models dietary tryptophan restriction modulated $B$ cell development and microbiota composition. In particular, we found a near-complete absence of B cell precursors in the bone marrow after dietary tryptophan restriction. The decline in $\mathrm{B}$ cell precursors was correlated with decreased abundance of the Akkermansia and Alistipes bacterial strains in the intestine. Thus, our results show that dietary tryptophan restriction is a powerful intervention to shape immunity and gut microbiota, also in aging. In chapter 8, we assessed the role of microbiota in the aging gut and immune system. Microbiota from young and aged mice were transferred to germfree mice. Aged microbiota induced higher $T$ helper-1 cell and regulatory $T$ cell frequencies in the spleen. In the ileum, the expression of inflammatory markers was increased after transferring aged microbiota, accompanied by differences in the abundance of microbial species. We conclude that senescent microbiota contribute to the inflammaging observed in aging mice.

In chapter 9, we discuss the findings presented in this thesis, concluding with directions for future research. In summary, our studies show that the aging gut and immune system of mice can be modulated by nutritional and/or microbial interventions. Interestingly, our mouse models clearly provide evidence that age-related effects could be reverted or prevented by these interventions. Nevertheless, our studies at the same time show the need for translational research in order to apply the presented dietary and microbial interventions in elderly. 


\section{Acknowledgements (dankwoord)}

Soli Deo Gloria. Het past me om als eerste God dank te zeggen voor alle nodige krachten om het werk voor dit proefschrift te doen. "Niet ons, o HEERE! niet ons, maar Uw Naam geef eer, om Uwer goedertierenheid, om Uwer waarheid wil." (Psalm 115:1)

Huub Savelkoul, bedankt dat je me met gezwinde spoed aangenomen hebt, halverwege maart 2011. Aangezien het TIFN GH002 project pas in oktober 2011 een beetje startte, ben ik daar extra dankbaar voor. Ik denk dat je mij uitzonderlijk veel vrijheid gegund hebt, in allerlei opzicht: samenwerkingen met Rotterdam, 'zijlijnprojecten' (bijv. de hoofdstukken over basofielen) en inzicht in de uitdagende financiële perikelen van ons project zowel in TIFN als WUR. Al deze aspecten hebben me verrijkt voor toekomstige uitdagingen. Bedankt voor alle goede gesprekken onderweg naar Rotterdam, Groningen of Zwolle, en niet te vergeten de wekelijkse wetenschappelijke meeting. Jouw enthousiasme en optimisme zullen me bijblijven. Harry Wichers, ook dank voor de steun in het proces, met name in het begin van het project. Paul de Vos, bedankt voor je rol als TIFN projectleider en je bevlogenheid in het onderzoek tussen jouw groep, $\mathrm{HMI}$ en CBI. Tijdens de enkele crisismeetings die we gehad hebben deze jaren, toonde je je een goede mediator. Ook dank voor je 'ADHD' aanvallen, waardoor je me soms dezelfde dag nog meerdere manuscripten gecorrigeerd terugstuurde. Pieter Leenen, wie had kunnen denken dat we zo nauw zouden samenwerken in deze jaren, toen ik in november 2011 je kantoor binnenstapte? Ik heb je leren kennen als een nauwgezette onderzoeker, de beste expert in myeloïde cellen die ik me kon wensen. In de loop der jaren is de samenwerking steeds verder opgevoerd, zodat je met recht op een heel aantal hoofdstukken als coauteur prijkt, en als copromotor zitting hebt in mijn promotiecommissie. Rudi Hendriks, onze samenwerking kwam pas op gang in 2013, toen ik naarstig op zoek was naar expertise op het gebied van B cellen. Dankzij je bereidwilligheid om inzicht te geven in jullie metingen, konden we een grote stap voorwaarts doen. Dank voor het openstellen van het lab, dank voor het vertrouwen. Ook je carrièreadviezen heb ik zeer gewaardeerd. Ik ben dan ook blij dat jij als tweede promotor in mijn commissie hebt willen toetreden. Marjolein de Jong, bedankt voor het delen van de protocollen en de dagen dat je me geholpen hebt op jullie lab. Genetici (Jan Hoeijmakers, Wilbert Vermeij en Renata Brandt), ik heb bewondering voor jullie nauwkeurige werkwijze, en ik bedank jullie voor de goede samenwerking. Edward Knol, bedankt voor de samenwerking op het werk over de basofielen.

All $\mathrm{CBI}, \mathrm{EZO}$ and $\mathrm{HMI}$ people, thanks for the cooperation all those years. Met name Ben, dank voor je interesse en ijver voor onze experimenten, en Edwin voor je betrokkenheid bij de start. Joost, dank voor je inzet voor het 'basofielenreview' en ook je latere belangstelling. Hilda, Sophie, Trudi, Marleen, bedankt voor jullie bereidheid om 
last-minute bestellingen te plaatsen en andere haastklussen uit te voeren. My roommates in the final period, Eva, Gosia, Marloes: it was a great pleasure to share the office with you. The same holds true for Danilo, and also thanks to you for the many "anti-frustrating" squash hours. Deze squash uren zijn later met eenzelfde doel voortgezet met Olaf en Jules, dank daarvoor! Marjolein Meijerink, dank voor je helpende hand en goede samenwerking! Dat geldt ook voor Linda Loonen, kort en krachtig in het experiment in INRA. Jerry Wells, thank you for your input in my project in the meetings we had in the last years, and for your confidence to perform flow cytometry in HMl experiments. Annick Mercenier, also many thanks for the discussions about our projects. Verder dank ik een aantal mensen van HAP (Annelies, Dorien, Evert, Hans, Inge), MIB (Clara, Floor, Hauke, Steven, Willem de Vos) en HNE (Mark). Veel dank aan alle medewerkers van het CKP voor de hulp bij alle experimenten (Bert, Frits, Judith, Lisette, Rene, Rob, Romy, Wilma). Zonder jullie hulp was een groot deel van de experimentele hoofdstukken geen feit geweest.

Thanks to all students that did a lot of work while performing their master thesis and/or internship with me: Martijn, Corine, Violeta, David, Chantal, Joanne, and Tim. All TIFN team members (including Maaike and Eelke), thanks for all you did those years. Marlies Elderman, dank voor het 'onderdak' op jullie lab in Groningen gedurende een periode in 2012-2013. Bruno Sovran, thank you for the cooperation in all experiments. Floor Hugenholtz, ook jij heel veel dank voor de samenwerking. Floris Fransen, we hebben maar kort kunnen samenwerken, maar ik heb genoeg gezien om respect voor je werkwijze te hebben. Dank voor de samenwerking! Thanks to all TIFN company experts that gave input during research and expert meetings, and to all TIFN theme assistants and financial controllers for printing posters and approving declarations. All others, with whom I pleasantly collaborated and discussed: thanks! Miriam van Dijk, dank voor de makkelijke wijze van samenwerken. Claudio Nicoletti, thank you for your confidence for hosting me for more than half a year at the Institute of Food Research (IFR). I will remember your positive attitude and your scientific approach. Good luck in Florence! Thanks to all the opponents that have read my thesis and participate in the discussion during my graduation.

Ben en papa, bedankt dat jullie als paranimf willen optreden tijdens mijn promotie. In brede zin druk ik mijn erkentelijkheid uit richting mijn familie en vrienden voor hun begrip en steun deze jaren. Papa en mama, bedankt voor de basis die jullie hebben gelegd in mijn jonge jaren.

Als laatste: Lia, heel erg bedankt voor alle steun. Je hebt een aantal dingen ingeleverd. Heel veel dank voor hulp die ik af en toe nodig had om frustraties uit te spreken richting wie dan ook. Lydia en Pieter, beiden geboren in deze periode: ik ben erg blij dat jullie ons leven verrijkt hebben. Het zorgde voor de nodige afleiding! 


\section{Curriculum vitae}

Adriaan Arend van Beek was born in Uddel (NL) on the 26th of May 1989. He graduated from high school Jacobus Fruytier (Apeldoorn) in 2006, after which he continued his education at Wageningen University (Wageningen, NL). In March 2011, he received his Master of Science degree (Nutrition and Health). During his master program, he performed a master thesis on pheochromocytoma at the department of Pathology in the Erasmus Medical Center (Rotterdam, NL) under supervision of Prof. Ronald de Krijger and Dr Esther Korpershoek. Subsequently, he performed an

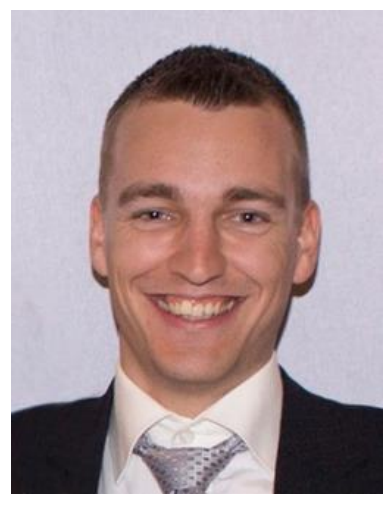
internship on the role of IL-12 and TSLP in the intestine at the department of Gut Health and Food Safety in the Institute of Food Research (IFR; Norwich, UK) under supervision of Prof. Claudio Nicoletti. In March 2011, Prof. Huub Savelkoul offered him a PhD position at the department of Cell Biology and Immunology of Wageningen University, funded by Top Institute of Food and Nutrition (Wageningen). Most of the findings of his $\mathrm{PhD}$ research can be found in this thesis, entitled: "The aging immune system and nutritional interventions". In 2012-2013, he worked as visiting scientist at the department of Pathology and Medical Biology of the University Medical Center of Groningen (NL) on a study on the effect of gender on (intestinal) immunology under supervision of Prof. Paul de Vos and Dr Marijke Faas. During most of his PhD research, he was visiting scientist at the department of Immunology of Erasmus Medical Center (under supervision of Dr Pieter Leenen) to study the role of microRNA and metabolism in aging macrophages. He also collaborated with the departments of Genetics (Prof. Jan Hoeijmakers and Dr Wilbert Vermeij) and Pulmonary Medicine (Prof. Rudi Hendriks). In the autumn of 2015, he received two small research grants to further study the effect of aging on intestinal immunology at the IFR. There, he was offered a postdoc position by Prof. Claudio Nicoletti for half a year, after which he returned to finalize his PhD thesis in Wageningen in April 2016. Since May 2016 he works as postdoc at the department of Gastroenterology and Hepatology in the Erasmus Medical Center with Dr Jaap Kwekkeboom and Dr Dave Sprengers, to study the role of costimulatory and co-inhibitory receptors on tumor-infiltrating leukocytes and their potential for immunotherapy in hepatocellular carcinoma and colorectal cancer. 


\section{List of publications}

1. P.B. Olthof, R.F. van Golen, B. Meijer, A.A. van Beek, R.J. Bennink, J. Verheij, T.M. van Gulik, M. Heger. Warm ischemia time-dependent variation in liver damage, inflammation, and function in hepatic ischemia/reperfusion injury. Biochimica et Biophysica Acta Molecular Basis of Disease 2017; 1863(2):375-385.

2. A.A. van Beek, F. Hugenholtz, B. Meijer, B. Sovran, O. Perdijk, W.P. Vermeij, R.M.C. Brandt, S. Barnhoorn, J.H.J. Hoeijmakers, P. de Vos, P.J.M. Leenen, R.W. Hendriks, H.F.J. Savelkoul. Tryptophan restriction arrests B cell development and enhances microbial diversity in WT and prematurely aging Ercc1-/ $\Delta 7$ mice. Journal of Leukocyte Biology 2017; 101:doi:10.1189/jlb.1HI0216.062RR.

3. A.A. van Beek, B. Sovran, F. Hugenholtz, B. Meijer, J.A. Hoogerland, V. Mihailova, C. van der Ploeg, C. Belzer, M.V. Boekschoten, J.H.J. Hoeijmakers, W.P. Vermeij, P. de Vos, J.M. Wells, P.J.M. Leenen, C. Nicoletti, R.W. Hendriks, H.F.J. Savelkoul. Supplementation with Lactobacillus plantarum WCFS1 prevents age-related decline of mucus barrier in colon of accelerated aging Ercc1-/ $\Delta 7$ mice. Frontiers in Immunology 2016; 7:408.

4. M. Elderman, A.A. van Beek, E.T. Brandsma, B. de Haan, H.F.J. Savelkoul, P. de Vos, M.M. Faas. Sex impacts Th1, Treg, and DCs in both the intestinal and systemic immunity in a mouse strain and location dependent manner. Biology of Sex Differences 2016; 7.

5. A.A. van Beek, J.A. Hoogerland, C. Belzer, P. de Vos, W.M. de Vos, H.F.J. Savelkoul, P.J.M. Leenen. Interaction of mouse splenocytes and macrophages with bacterial strains in vitro: the effect of age in the immune response. Beneficial Microbes 2016; 7(2): 275-287.

6. M. Broekgaarden, M. Kos, F.A. Jurg, A.A. van Beek, T.M. van Gulik, M. Heger. Inhibition of NF-KB in Tumor Cells Exacerbates Immune Cell Activation Following Photodynamic Therapy. International Journal of Molecular Science 2015; 16(8): 1996019977.

7. A.A. van Beek, E.F. Knol, P. de Vos, M.J. Smelt, H.F.J. Savelkoul, R.J.J. van Neerven. Recent Developments in Basophil Research: Do Basophils Initiate and Perpetuate Type 2 T-helper Cell Responses? International Archives of Allergy and Immunology 2013; 160(1): 7-17.

\section{Submitted or in preparation}

1. A.A. van Beek, F. Fransen, B. Meijer, P. de Vos, E.F. Knol, H.F.J. Savelkoul. Basophil phenotype and function is determined by gender and microbiota. In preparation.

2. A.A. van Beek, T. Borghuis, B. Meijer, H.F.J. Savelkoul, R.W. Hendriks, P. de Vos,

F. Fransen. Gut microbiota repress B cell development. In preparation. 
3. A.A. van Beek, F. Fransen, B. Meijer, P. de Vos, E.F. Knol, H.F.J. Savelkoul. Basophil differentiation changes during aging in mice under the influence of senescing microbiota. Submitted.

4. A.A. van Beek, J. van den Bossche, P.G. Mastroberardino, M.P.J. de Winther, P.J.M. Leenen. Metabolic and epigenetic alterations in aging macrophages: a recipe for inflammaging? Submitted.

5. F. Fransen, A.A. van Beek, T. Borghuis, S. El Aidy, F. Hugenholtz, C. van der Gaast-de Jongh, H.F.J. Savelkoul, M.I. De Jonge, M.V. Boekschoten, H. Smidt, M.M. Faas, P. de Vos. Aged gut microbiota contribute to systemical inflammaging. Submitted.

6. F. Fransen, A.A. van Beek, T. Borghuis, B. Meijer, F. Hugenholtz, C. van der Gaast-de Jongh, H.F.J. Savelkoul, M.I. De Jonge, M.M. Faas, M.V. Boekschoten, H. Smidt, S. El Aidy, P. de Vos. The impact of gut microbiota on gender-specific differences in immunity. Submitted

7. R.F. van Golen, M.J. Reiniers, G. Marsman, L.K. Alles, D.M. van Rooyen, B. Petri, V.A. van der Mark, A.A. van Beek, B. Meijer, M.A. Maas, B.M. Luken, J. Verheij, G.C. Farrell, S. Zeerleder, N.C. Teoh, T.M. van Gulik, M.P. Murphy, P. Kubes, M. Heger. Mitochondrial reactive oxygen species trigger hepatic ischemia/reperfusion injury by inducing the release of high-mobility group box 1. Submitted.

8. M.J. Reiniers, P.B. Olthof, R.F. van Golen, M. Heger, A.A. van Beek, B. Meijer, R. Leen, A.B.P. van Kuilenburg, B. Mearadji, R.J. Bennink, J. Verheij, T.M. van Gulik. Hypothermic perfusion with retrograde outflow improves early liver generation following (extended) right hemihepatectomy; results of a randomized controlled trial. Submitted.

9. B. Sovran, F. Hugenholtz, M. Elderman, A.A. van Beek, K. Graversen, M. Huijskes, M.V. Boekschoten, M. Kleerebezem, H.F.J. Savelkoul, P. de Vos, J. Dekker, J.M. Wells. Intestinal barrier impairment in ageing mice predispose to "inflammageing" in the intestinal mucosa. In revision.

10. M. Elderman, F. Hugenholtz, C. Belzer, M.V. Boekschoten, A.A. van Beek, B. de Haan, H.F.J. Savelkoul, P. de Vos, M.M. Faas. Sex and strain dependent differences in intestinal immunity correlated with differences in microbiota diversity. In revision.

\section{Publications in refereed, non-English journals}

A.A. van Beek, E.F. Knol, R.J.J. van Neerven, H.F.J. Savelkoul. Basofiele granulocyten als effectorcellen. Nederlands Tijdschrift Allergie \& Astma 2013; 13(1):27-34. Republished in Tijdschrift voor Belgische Laboratorium Technologen 2014; 41(1): 9-19. 


\section{Overview of completed training activities}

National and international conferences ${ }^{*}$ (10 credits)

$8^{\text {th }}$ NuGoweek, Wageningen

2011

$3^{\text {rd }}$ TNO Beneficial Microbes Conference, Noordwijkerhout 2012

$43^{\text {rd }}$ Annual Scientific Meeting ASI, Wellington, New Zealand (P) 2013

$3^{\text {rd }}$ International Summer Frontiers Symposium, Nijmegen (L) 2014

$7^{\text {th }}$ Seeon Conference, Seeon, Germany (P) 2014

$4^{\text {th }}$ European Congress of Immunology, Vienna, Austria (O) 2015

\section{Seminars and workshops ${ }^{*}$ ( 31 credits)}

TIFN Research and Expert Meetings, Wageningen/Zwolle/Amersfoort (4x 0, 1x P)

2011-2015

Mini-symposium 'How to write a world class paper', Wageningen

2011

$4^{\text {th }}$ Symposium on Mucosal Immunology, Rotterdam

2011

$2^{\text {nd }}$ Annual Conference TIFN, Fort Groenekan

2011

$13^{\text {th }}$ Gut Day, Wageningen

2011

Unit Meeting Immunology, Rotterdam (2x 0)

2012-2013

$3^{\text {rd }}$ Annual Conference TIFN, Papendal

2012

Course on philosophy of science and ethics

2012

TIFN WE Days, Ameland (0)

2012

Mini-symposium 'Mucosal factors regulating allergy', Wageningen

2013

Danone International Scientific Symposium, Doorwerth

2013

WIAS Introduction Course, Wageningen

2013

NVVI Symposium, Lunteren

2014

WIAS Science Day, Wageningen ( 0 )

2014

$5^{\text {th }}$ Annual Conference TIFN, Utrecht

2014

TIFN WE Days, Apeldoorn (O)

2014

$16^{\text {th }}$ Gut Day, Amsterdam (P)

2014

NVVI 50 ${ }^{\text {th }}$ Anniversary Symposium, Kaatsheuvel (2x P)

2014

NVVI Symposium, Lunteren

2015

$6^{\text {th }}$ Symposium on Mucosal Immunology, Rotterdam (2x 0)

2015

Seminar Institute of Food Research, Norwich, United Kingdom (0)

2015

Coffee Break Science, Norwich, United Kingdom (0)

2016

Departmental presentation Immunology, Rotterdam (0)

2016

\footnotetext{
*Poster presentations indicated with "P", oral presentations with "O", laptop presentation with " $L$ ".
} 
Disciplinary and interdisciplinary courses (6 credits)

$12^{\text {th }}$ Fish Immunology Workshop, Wageningen

In vivo imaging: from molecule to organism, Rotterdam 2011

Advanced course Immunology, Utrecht 2012

BD course flow cytometry, Erembodegem, Belgium 2012

TIFN IP workshop, Wageningen 2012

Course Advanced visualization of omics data, Wageningen 2014

Professional skills support courses ( 3 credits)

PhD competence assessment, Wageningen 2011

PhD career assessment, Wageningen 2015

Techniques for writing and presenting scientific papers, Wageningen 2015

PhD peer consultation, Wageningen 2015

\section{Research skills training (7 credits)}

External training period, Erasmus MC, Rotterdam 2012-2015

External training period, UMCG, Groningen 2012-2013

Course 'blood collection via cheek puncture', Erasmus MC, Rotterdam 2014

External training period, Radboud UMC, Nijmegen 2015

External training period, Institute of Food Research, Norwich, UK 2015

\section{Didactic skills training (26 credits)}

Immunomodulation by Food and Feed, practical 2011

Cell Biology I, tutorship 2011

Research Master Cluster, evaluation of proposal 2012-2014

Development and Healthy Ageing, lecture and practical 2013-2014

Supervision of 7 MSc students, 3 capita selecta 2013-2015

\section{Total study load: 83 ECTS}


The study presented in this thesis was performed within the framework of Top Institute of Food and Nutrition.

Financial support from Wageningen University, Nestlé, Top Institute of Food and Nutrition, Winclove Probiotics, Yakult Nederland B.V., VitalHealth Solutions B.V., BD Biosciences, Visser \& Visser Accountants Belastingadviseurs, Pedicuresalon Rita, Factsonline hazardous material accident database, and VBI Ltd. NZ for printing this thesis is gratefully acknowledged.

Cover design by Lia van Beek

Printed by Digiforce 

